\title{
Categorias Modeláveis
}

Hugo Luiz Mariano

Dissertação apresentada

ao

Instituto de Matemática e Estatística

da

Universidade de São Paulo

para a obtenção do grau de mestre

em

Matemática

Área de concentração : Lógica e fundamentos

Orientador : Prof. Dr. Odilon Otávio Luciano

Durante a elaboração deste trabalho, o autor recebeu apoio financeiro do CNPq

Sร̃ก Paulo. agosto de 1997 - 


\section{Categorias Modeláveis}

Este exemplar corresponde à versão final da dissertação defendida por Hugo Luiz Mariano em 27 de agosto de 1997 e aprovada pela comissão julgadora composta por:

- Prof. Dr. Odilon Otávio Luciano (IME-USP)

- Prof. Dr. Edward Hermann Haeusler (DI-PUC)

- Prof. Dr. Newton Carneiro Affonso da Costa (FFLCH-USP) 


\title{
Dedicatória - Agradecimentos
}

À Beth, Zé, Dé e Toto pelo carinho, retaguarda e paciência simplesmente infinitos.

Ao Odilon, já um amigo da família, pela orientação e confiança neste trabalho.

Aos amigos, funcionários, professores e CPG do IME-USP por suas gentilezas.

Ao Mr. Paul "da Viola" Krause, a quem freqüentemente recorria para a obtenção de obras não disponíveis.

Às pessoas que participaram diretamente deste trabalho e que não hesitei em abusar de sua cortesia:' o Tonico, que organizou as referências bibliográficas; o Fernando, que me socorreu diariamente contra "O Computador"; o Zé, que sempre dispunha de bons conselhos, que realizou a "debugagem" do texto e que, conjuntamente com o Saltini, o Eliezer e o Fernando, criaram os diagramas. Obrigado!

E àquelas que, de modo menos direto mas igualmente importante, me auxiliaram neste período.

Estarei sempre em dívida com vocês.

\author{
Hugo Luiz Mariano
}




\section{Conteúdo}

Introdução

1 Preliminares $\quad 4$

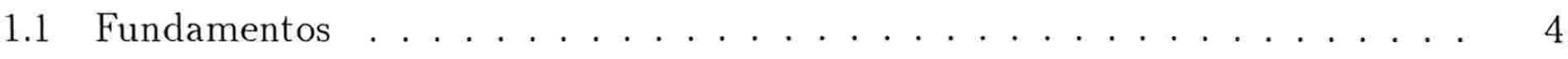

1.1.1 Axiomas de NBG . . . . . . . . . . . . . . . . . . 4

1.1 .2 Ordinais . . . . . . . . . . . . . . . . 5

1.1.3 Aritmética cardinal . . . . . . . . . . . . . 6

1.1.4 Grandes cardinais . . . . . . . . . . . . . . . . 6

1.1 .5 Universos . . . . . . . . . . . . . . . . . . . 7

1.2 Fatos básicos sobre categorias . . . . . . . . . . . . . . . 11

1.2.1 Grafos e categorias ................... 11

1.2.2 Categorias livres, quocientes e diagramas comutativos . . . . . . . 18

1.2 .3 Cones . . . . . . . . . . . . . . . . . . . 20

1.2.4 O funtor "componentes conexas" e funtores finais/iniciais . . . . . . 25

1.3 Mais categorias! . . . . . . . . . . . . . . . . . . . 27

1.3 .1 Adjunção . . . . . . . . . . . . . . . . . 27

1.3.2 Comutação de limites . . . . . . . . . . . . . . . . . . . . 34

1.3.3 Completamentos indutivos . . . . . . . . . . . . . 40

2 Categorias acessíveis $\quad \mathbf{5 2}$

$\begin{array}{lll}3 & \text { Esboços } & 61\end{array}$

4 Lógicas infinitárias $\quad 75$

$5 \quad$ Categorias axiomatizáveis, esboçáveis e acessíveis $\quad 89$

5.1 Categorias esboçáveis são axiomatizáveis . . . . . . . . . . . . . . . . . . . . . . . . . .

5.2 Categorias axiomatizáveis são esboçáveis . . . . . . . . . . . . . . . . . . . . . . . . . . . . . . . . .

5.3 Categorias esboçáveis são acessíveis . . . . . . . . . . . . . . . . . . . 92

5.4 Categorias acessíveis são esboçáveis . . . . . . . . . . . . . . . 94 
5.5 Considerações adicionais . . . . . . . . . . . . . . . . . 96

6 Miscelânea $\quad 101$

$\begin{array}{lr}\text { Bibliografia } & 108\end{array}$ 


\section{Introdução}

Esta dissertação segue na mesma direção dos trabalhos de Birkhoff $([035])$ : que correlacionam teorias equacionais e propriedades categoriais $(H, S, P)$ e, mais modernamente, com as idéias de Lawvere ([152]); para quem teorias são categorias com propriedades e modelos são funtores que preservam essas propriedades.

No capítulo 1 , após introduzirmos uma fundamentação conjuntista suficientemente maleável para as suportar as construções da teoria das categorias, colocamos os fatos básicos sobre categorias que futuramente serão utilizados e outros resultados menos clássicos que culminam com a noção de completamentos indutivos de categorias.

Os objetos enfocados no capítulo 2 são instâncias dos considerados no capítulo inicial que se destacam pela possibilidade da "variação de estágio".

No capítulo 3 apresentamos a noção de esboço ; introduzida por Ehresmann ([078]) ; que são "especificações" de limites projetivos e indutivos em categorias.

Colocamos no capítulo 4 as noções tradicionais das lógicas infinitárias procurando realçar o aspecto categorial embutido nas construções .

Interconectamos, no capítulo 5 , as idéias introduzidas anteriormente e adicionamos alguns resultados.

O capítulo 6 é uma coletânea de exemplos, contra-exemplos, comparações com noções alternativas de algebricidade, e resultados sobre as categorias em questão .

$\mathrm{Na}$ bibliografia enumeramos obras contendo assuntos correlatos e que estão disponíveis na biblioteca do IME - USP , com a importante exceção da coleção Diagrammєs .

Apesar de não utilizarmos no texto, em momento algum, o termo "categoria modelável" o título se justifica : categorias axiomatizáveis são categorias de modelos de sentenças : categorias esboçáveis são categorias de modelos de esboços e "catégories modelables" é o

termo originalmente introduzido por Lair em [145] para designar nossas acessíveis. 


\section{Capítulo 1}

\section{Preliminares}

\subsection{Fundamentos}

As referências para esta seção ; e para toda terminologia e resultados de teoria dos conjuntos utilizados neste trabalho, são: [075] e [117]: para o último ítem (universos) devemos acrescentar [098] e, particularmente, seu apêndice.

Dentre as várias possíveis fundamentações para a teoria das categorias, nos parecem mais cômodas aquelas nas quais podemos falar de "totalidades" sem constrangimentos. Para isso uma teoria de classes e conjuntos é suficiente. Contudo, há construções categoriais bastante corriqueiras (e.g. categorias de funtores) que ficam limitadas com apenas a distinção entre classes próprias e impróprias. Uma solução natural para isso é "estratificar" o universo dos conjuntos de modo que as categorias em um dado estágio da hierarquia possuam propriedades análogas às da categoria total e que construções entre categorias em um dado estágio possam ser efetuadas (efetivadas) em um estágio eventualmente superior. Formalizaremos, um pouco, isso.

\subsubsection{Axiomas de NBG}

Nossa teoria básica será NBG : uma teoria de primeira ordem com igualdade cuja linguagem contém apenas o símbolo relacional binário $\in$ e onde denotamos as variáveis, indistintamente, por letras minúsculas e maiúsculas: $x, y, X, Y^{\prime}, \ldots$

Definição 1 :

(i) $S(x) \Longleftrightarrow \exists y(x \in y)$ (" $x$ é um conjunto") .

(ii) $x \subseteq y \Longleftrightarrow \forall z(z \in x \rightarrow z \in y)(\Longleftrightarrow \forall z(S(z) \wedge z \in x \rightarrow z \in y))$.

Axiomas de NBG

(i)Axioma da extensionalidade : $(x \subseteq y \wedge y \subseteq x) \rightarrow x=y$. 
(ii)Esquema da compreensão : $\exists y \forall x(x \in y \leftrightarrow S(x) \wedge \varphi(x))$, onde $y$ não ocorre livre em $\varphi(x)$ e as quantificações em $\varphi(x)$ são limitadas. (Notemos que, pelo axioma da extensionalidade, $y$ está univocamente determinado : notação $y=\{x: S(x) \wedge \varphi(x)\}$.)

\section{Definição 2 :}

(i) $V=\{x: S(x) \wedge x=x\}$.

(ii) $\emptyset=\{x: x \in V \wedge x \neq x\}$.

(iii) $x, y \in V \Rightarrow\{x, y\}=\{z \in V: z=x \vee z=y\}$.

(iv) $\cup x=\{z \in V: \exists y(z \in y \in x)\}$.

(v) $\mathcal{P}(x)=\{y \in V: y \subseteq x\}$.

(vi) $\operatorname{In} f(x) \Longleftrightarrow(\emptyset \in x \wedge \forall y(y \in x \rightarrow y \cup\{y\} \in x))$.

(vii) $f: x \rightarrow y \Longleftrightarrow f$ é uma função de domínio $x$ e codomínio $y$.

(viii) $c h(f, x) \Longleftrightarrow(f:(\mathcal{P}(x)-\emptyset) \rightarrow x) \wedge \forall y(y \in \operatorname{dom}(f) \rightarrow f(y) \in y)$.

Axiomas de NBG (continuação )

(iii) Axioma da regularidade : $x \neq \emptyset \rightarrow \exists y(y \in x \wedge x \cap y=\emptyset)$.

(iv) Axioma do vazio : $\emptyset \in V$.

(v)Axioma do par : $x, y \in V \rightarrow\{x, y\} \in V$.

(vi)Axioma da reunião : $x \in V \rightarrow \cup x \in V$.

(vii)Axioma das partes : $x \in V \rightarrow \mathcal{P}(x) \in V$.

(viii)Axioma do infinito: $\{x \in V: \operatorname{In} f(x)\} \neq \emptyset$.

(ix)Axioma da substituição : $(f: x \rightarrow V \wedge x \in V) \rightarrow f_{*}(x) \in V$; onde $f$ é uma função de domínio $x$, codomínio $V$ e imagem $f_{*}(x)$.

(x)Axioma da escolha (global) : $\exists f(\operatorname{ch}(f, V))$.

Observação 1 :

(i) Assumiremos o axioma da escolha em todas as teorias de conjuntos (classes) que considerarmos; assim denotaremos por ZF a usual ZFC e NBG a NBG + C (global).

(ii) Utilizaremos resultados (aritmética cardinal,hierarquia de Lévy;...) provados, nas referências, em ZF pois NBG e ZF "dizem as mesmas coisas sobre os conjuntos".

\subsubsection{Ordinais}

Ordinais $\left(\alpha, \beta, \alpha^{\prime}, \alpha_{i}, \ldots\right)$ são os ordinais de Von Neumann; cardinais $\left(\lambda, \kappa, \kappa^{\prime}, \ldots\right)$ são ordinais iniciais: $O R D$ é a classe bem ordenada dos ordinais; $C A R D \subseteq O R D$ a classe dos cardinais.

O axioma da escolha (princípio da boa ordenação ) nos garante que todo conjunto é isomorfo (equipotente) a um (único) cardinal. Notação : $x \in V \Rightarrow \# x \in C A R D$.

Seja $O R D^{+}=O R D \cup\{\infty\}$ a classe bem ordenada, extensão de $O R D$, tal que $\propto \notin O R D$ $\left(\propto=\{\emptyset\}\right.$. por exemplo) e $\forall \alpha \in O R D(\alpha<\infty)$. Analogamente definimos $C A R D^{+}=$ 
$C A R D \cup\{\infty\}$ e convencionamos que $\# x=\propto \Longleftrightarrow x$ é classe própria; deste modo o axioma da substituição nos diz que $\propto$ é um "cardinal regular".

Definimos, por indução em $\alpha \in O R D^{+}$: a seqüência (hierarquia de Von Neumann):

. $\alpha=0 \Rightarrow V_{\alpha}=$ :

$\exists \beta(\alpha=\beta+1) \Rightarrow V_{\alpha}=\mathcal{P}\left(V_{\beta}\right)$;

. $\alpha=\mathrm{V}\left[0, \alpha\left[\neq 0 \Rightarrow V_{\alpha}=\cup_{\beta<\alpha} V_{\beta}\right.\right.$ (em particular, $V_{\infty}=\cup_{\beta \in O R D} V_{\beta}$ ).

$\mathrm{O}$ axioma da regularidade implica (e equivale se assumimos os outros axiomas) a $V=V_{\infty}$ ; definimos então $\forall x \subseteq V\left(\rho(x)=\min \left\{\alpha \in O R D^{+}: x \subseteq V_{\alpha}\right\}\right)$.

Proposição 1 : Temos que:

(i) $\forall \alpha \in O R D^{+}\left(V_{\alpha}\right.$ é transitivo ).

(ii) $\forall \alpha \in O R D(\rho(\alpha)=\alpha)$.

(iii) $\forall \alpha \in O R D^{+}\left(V_{\alpha}=\{x \in V: \rho(x)<\alpha\}\right)$.

(iv) $\forall x(x \in V \Longleftrightarrow \rho(x)<\infty)$.

(v) $\forall x, y(x \in y \subseteq V \Rightarrow \rho(x)<\rho(y) \leq \infty)$.

(vi) $\forall x(x \in V \Rightarrow \rho(x)=\bigvee\{\rho(y)+1: y \in x\})$.

\subsubsection{Aritmética cardinal}

\section{Definição 1}

(i) Sejam $X \in V$ e $\lambda \in C A R D$, denotaremos por $[X]^{=\lambda}$; $[X]^{<\lambda}$ e $[X]^{\leq \lambda}$ os subconjuntos de $\mathcal{P}(X)$ de cardinalidades, respectivamente, $=\lambda:<\lambda$ e $\leq \lambda$.

(ii) Sejam $\mu, \lambda \in C A R D: \mu^{<\lambda}=\bigvee\left\{\mu^{\nu}: \nu \in C A R D \wedge \nu<\lambda\right\}$.

Proposição 1 : Temos os seguintes resultados:

(i) Seja $\left\{\mu_{i}: i \in I\right\} \subseteq(C A R D-\{0\})$ tal que $\exists i \in I\left(\mu_{i} \geq \omega\right)$, então $\sum_{i \in I} \mu_{i}=$ $\# I . \bigvee_{i \in I} \mu_{i}$.

(ii) Sejam $X \in V$ e $\lambda \in(C A R D-\{0\})$ tais que $\omega+\lambda \leq \# X$; então:

. $\#[X]^{=\lambda}=(\# X)^{\lambda}$.

. $\#[X]^{<\lambda}=\sum\left\{(\# X)^{\nu}: \nu \in C A R D \wedge \nu<\lambda\right\}=(\# X)^{<\lambda}$.

(iii) Dados $\omega \leq \lambda \leq \mu<\infty$; temos:

. $\nu<\lambda \Rightarrow\left(\mu^{<\lambda}\right)^{\nu}=\mu^{<\lambda}$.

. $\left(\mu^{<\lambda}\right)^{<\lambda}=\mu^{<\lambda}$.

\subsubsection{Grandes cardinais}

Cardinais "grandes" são aqueles a partir dos quais podemos obter conjuntos transitivos que são modelos de $Z F$ ( $N B G$ ). Segue do teorema (3.5.10) em [Drake] que a existência destes 
cardinais nào pode ser demonstrada a partir dos axiomas da teoria se esta for consistente. Necessitaremos de alguns deles no trabalho.

Definição 1 : Seja $\lambda \in C A R D^{+}$regular. $f:[0, \lambda[\rightarrow[0, \lambda[$ é uma $\lambda$-função normal $\Longleftrightarrow$ : . $\forall 0 \leq \alpha<\beta<\lambda(f(\alpha)<f(\beta))$ ( $f$ é crescente) .

. $\forall 0 \leq \alpha<\lambda\left(\alpha=\bigvee\left[0, \alpha\left[\Rightarrow f(\alpha)=\bigvee_{\beta<\alpha} f(\beta)\right)\right.\right.$ ( $f$ é contínua) .

Observação 1 :

(i) $f:[0, \lambda[\rightarrow[0, \lambda[$ função normal $\Rightarrow \forall \alpha \in[0, \lambda[(\alpha \leq f(\alpha))$ ( $f$ é inflacionária) .

(ii) As funções normais mais importantes são $\aleph, \beth:[0, \infty[\rightarrow[0, \infty[$ onde:

. $\aleph: \aleph$ contínua $; \aleph_{0}=\omega: \aleph_{\alpha+1}=\left(\aleph_{\alpha}\right)^{+}$.

. $\beth:$ contínua $; \beth_{0}=\omega ; \beth_{\alpha+1}=\# \operatorname{Func}\left(\beth_{\alpha}, 2\right)$.

Proposição 1 : Se $f$ é função normal de domínio $O R D=[0, \infty[$ e imagem contida nos cardinais infinitos e $\kappa$ é um cardinal regular então $f(\kappa)=\kappa \Longleftrightarrow \exists \alpha=\cup \alpha \neq 0(f(\alpha)=\kappa$ ).

\section{Definição 2 :}

(i) Denominamos cardinais limites (limites fortes) as imagens por $\aleph(\beth)$ de ordinais limites , 0 incluso.

(ii) Cardinais (fortemente) inacessíveis s ao cardinais $\kappa>\omega$ que são regulares e limites fortes.

(iii) Cardinais mensuráveis são cardinais $\kappa>\omega$ tais que $(\mathcal{P}(\kappa), \subseteq)$ admite algum ultrafiltro, não principal e $<\kappa$-completo.

(iv) Cardinais (fortemente) compactos são cardinais regulares $\kappa>\omega$ tais que para todo conjunto $X$; todo filtro próprio $<\kappa$-completo em $(\mathcal{P}(X)$. $\subseteq$ ) está contido em algum ultrafiltro $<\kappa$-completo.

Proposição $2: \kappa$ compacto $\Rightarrow \kappa$ mensurável $\Rightarrow \kappa$ inacessível.

\subsubsection{Universos}

Definição 1: $U \in V$ é um universo se e somente se $U$ satisfaz:

(i)Transitividade : $\forall x, y(y \in x \in U \rightarrow y \in U)$.

(ii)Vazio : $\emptyset \in U$.

(iii)Par : $\forall x, y(x, y \in U \rightarrow\{x, y\} \in U)$.

(iv)Reunião : $\forall x(x \in U \rightarrow \cup x \in U)$.

(v)Partes: $\forall x(x \in U \rightarrow \mathcal{P}(x) \in U)$.

(vi)Infinito: $\{x \in U: \operatorname{Inf}(x)\} \neq \emptyset$.

(vii)Substituição: $\forall x \forall f\left((f: x \rightarrow U \wedge x \in U) \rightarrow f_{*}(x) \in U\right)$.

Proposição 1 : Se $U \in V$ é universo então: 
(i) $x \in U \Rightarrow\{x\} \in U$. (par)

(ii) $x, y \in U \Rightarrow(x, y) \in U$. ( (i) + par )

(iii) $I \in U \wedge\left\{x_{i}: i \in I\right\} \subseteq U \Rightarrow \cup_{i \in I} x_{i} \in U$. (subst. + reunião)

(iv) $X, Y \in U \Rightarrow X \times Y \in U$. ( (ii)+(iii))

(v) $(x \in U \wedge y \subseteq x) \Rightarrow y \in U$. (partes+trans.)

(vi) $(X \in U \wedge R$ relação de equivalência em $X) \Rightarrow(R \in U \wedge X / R \in U)$. ((iv)+(v) ; partes $+(\mathrm{v}))$

(vii) $\left(I \in U \wedge\left\{x_{i}: i \in I\right\} \subseteq U\right) \Rightarrow \bigsqcup_{i \in I} x_{i} \in U .\left(\bigsqcup_{i \in I} x_{i} \subseteq I \times \cup_{i \in I} x_{i}\right)$

(viii) $X, Y \in U \Rightarrow X^{Y}=\{f: X \rightarrow Y: f$ é função $\} \in U .\left(X^{Y} \subseteq\{X\} \times \mathcal{P}(X \times Y) \times\{Y\}\right.$

(ix) $\left(I \in U \wedge\left\{x_{i}: i \in I\right\} \subseteq U\right) \Rightarrow \prod_{i \in I} x_{i} \in U .\left(\prod_{i \in I} x_{i} \subseteq\left(\cup_{i \in I} x_{i}\right)^{I}\right)$

(x) $x \in U \Rightarrow \# x<\# U$. (por trans. \#x $\leq \# U$ e por partes $\# x<\# \mathcal{P}(x) \leq \# U$ )

Proposição 2: Sejam $U \in V$ universo e $c(U)=\bigvee_{x \in U} \# x$. Então:

(i) $c(U) \leq \# U$. (trans.)

(ii) $x \in U \Rightarrow \# x<c(U)$. (partes)

(iii) $\lambda<c(U) \Rightarrow \exists x(x \in U \wedge \# x=\lambda)$.

( (1.v))

(iv) $c(U)>\omega \cdot($ infi. + (ii) )

(v) $\lambda<c(U) \Rightarrow 2^{\lambda}<c(U)$. ( (iii)+partes+(ii))

(vi) $\forall\left\{\mu_{\alpha}: \alpha \in \lambda\right\}\left(\lambda<c(U) \wedge \forall \alpha \in \lambda\left(\mu_{\alpha}<c(U)\right) \Rightarrow \sum_{\alpha \in \lambda} \mu_{\alpha}<c(U)\right)$. ( (iii) $+(1 . v i i)+($ ii) $)$

(vii) $c(U)$ é inacessível. ( (iv) $+(\mathrm{v})+($ vi) )

Proposição 3: Existe isomorfismo entre as classes ordenadas dos universos $(U N I V, \subseteq)$ e dos cardinais inacessíveis $(I N A C, \leq)$.

Vamos verificar que a aplicação (crescente) $U \mapsto c(U)$ é inversível.

Segue do parágrafo 1 do capítulo 4 em [075] que $\kappa \in I N A C \Rightarrow V_{\kappa} \in U N I V$.

Do corolário 1 do teorema 2 do apêndice de [098] e do axioma da regularidade segue que:

. $U=\mathcal{H}(c(U))=\{x \in V: \# T C(x)<c(U)\}$. (veja [075] ; cap.3 ; par.7)

. $c(U)=\# U$.

Logo basta mostrar que $\kappa \in I N A C \Rightarrow\left(\kappa=\# V_{\kappa} \wedge \mathcal{H}(\kappa)=V_{\kappa}\right)$.

. $\kappa=\# V_{\kappa}$.

Por indução em $\alpha \in O R D$ temos $\# \alpha \leq \# V_{\alpha} \leq \# V_{\omega+\alpha}=\beth_{\alpha} \operatorname{logo}$ : para $\kappa \in I N A C$; $\kappa \leq \# V_{\kappa}=\beth_{\kappa}=\kappa$.

. $\mathcal{H}(\kappa)=V_{\kappa}$. 
De [075] ; cap.3 ; par. $\mathbf{\imath}$ temos que para todo cardinal $\mu \geq \omega\left(\mathcal{H}(\mu) \subseteq V_{\mu}\right) . \mathcal{H}(\kappa) \supseteq V_{\kappa}$ pois $x \in V_{\kappa} \Rightarrow \rho(x)<\kappa \Rightarrow x \subseteq V_{\rho(x)} \subseteq V_{\omega+\rho(x)} \Rightarrow \# x \leq \beth_{\rho(x)}<\beth_{\kappa}=\kappa$ e porque $\forall x$ ( $\left.x \in V_{\kappa} \Longleftrightarrow T C(x) \in V_{\kappa}\right)$ pois $V_{\kappa} \in U N I V$.

Observação 1 : Da definição 1 e de [Drake] . cap.4 par.1 segue que se $U$ é universo então $\left(U, \in_{I_{U}}\right)$ é modelo transitivo de $Z F$ e que $\left(U, \mathcal{P}(U), \in I_{\mathcal{P}(U)}\right)$ é modelo de $N B G$ (com "escolhas globais" !) portanto; pela proposição 3 e o comentário no início da seção anterior temos que os cardinais inacessíveis, mensuráveis e compactos "são" grandes cardinais e que não podemos mostrar em $Z F(N B G)$ que as classes COMP, MENS, INAC,UNIV são não vazias.

Observação 2 : Da definição 1 e da proposição 1 temos que se $\mathcal{G}=\left(G, . \cdot()^{-1}, \epsilon\right)$ é um grupo e $U$ é um universo então $\mathcal{G} \in U \Longleftrightarrow G \in U$; além disso se $\mathcal{G}$ e $\mathcal{H}$ são grupos e $f: G \rightarrow H$ é homomorfismo de $\mathcal{G}$ em $\mathcal{H}$ : i.e. ${ }_{\mathcal{G}} f_{\mathcal{H}}=(\mathcal{G}, f, \mathcal{H}) \in G R(\mathcal{G}, \mathcal{H})$; então $\mathcal{G} f_{\mathcal{H}} \in U \Longleftrightarrow G, H \in U$. Fenômenos semelhantes ocorrem quanto consideramos espaços topológicos e funções contínuas, anéis e homomorfismos de anéis; módulos sobre um anel $\mathcal{R} \in U$ e transformações $\mathcal{R}$-lineares e, em geral, para estruturas com morfismos no sentido de Bourbaki, com "échelon" baseado em $n$ conjuntos principais e $m$ conjuntos auxiliares com $n, m<\omega, n>0$ ([043] cap.4). Assim temos que $U-S E T \hookrightarrow S E T$; $U-$ $G R \hookrightarrow G R ; U-T O P \hookrightarrow T O P: U-_{\mathcal{R}} M O D \hookrightarrow_{\mathcal{R}} M O D(\mathcal{R} \in U-A N) \ldots$ são inclusões plenas de subcategorias pequenas ( $\subseteq U$, portanto conjuntos) em categorias "grandes" (classes próprias). Notemos que $U-C A T \hookrightarrow C A T$ (onde $C A T$ é a categoria das categorias pequenas $(\in V)$ e $U-C A T$ é a categoria das categorias $\in U)$ mas ;assim como SET, GR, TOP, ... $\notin$ obj $(C A T)$ temos $U-S E T, U-G R, U-T O P: \ldots \notin o b j(U-C A T)$.

Isto pode ser reparado:

Fundamentaremos a teoria das categorias em $N B G U N=N G B+\forall x \in V \exists U(x \in U \wedge$ $U \in U N I V)$.

\section{Observação 3 :}

(i) $N B G U N$ é equivalente a $N B G I N=N B G+(I N A C$ é classe própria) .

(ii) $N B G U N, N B G I N$ são extensões próprias de $N B G$ se esta for consistente.

(iii) A classe (própria) dos universos está bem ordenada pela inclusão e se $x \in V$ então existe o menor universo $U n[x]$ tal que $x \in U n[x]$.

(iv) Se $W \in U N I V$ e $s(W)=U n[W]$ temos que $W-S E T$, $W-G R, W-T O P, W-$ $C A T,(W-T O P)^{W-G R} ; \ldots \in \operatorname{obj}(s(W)-C A T)$.

Portanto podemos mudar de "estágio" sempre que for preciso. Notemos que os "estágios" são "representativos":

Proposição 4 : Sejam $U \subseteq W, U \in U N I V$ e $W \in U N I V$ ou $W=V$ então $U-S E T$ é fechado em $W-S E T$, por limites projetivos/indutivos canônicos e $U$-pequenos (i.e., limites 
proj./ind. de funtores $F: I \rightarrow U-S E T$ onde $I \in \operatorname{obj}(U-C A T))$.

Segue da construção canônica de limites proj. (ind.) em SET como subconjuntos de produtos cartesianos (quocientes de reuniões disjuntas) e dos itens (1.v) e (1.ix) ( (1.vi) e (1.vii) ) da proposição 1 .

Observação 4: Fato semelhante ocorre com os limites proj. (ind.) nas categorias "familiares à Matemática". Notemos também que os conjuntos numéricos $\mathbb{N}, \mathbb{Z} ; \mathbb{Q}, \mathbb{R}, \mathbb{C}$ : com suas construçôes usuais, pertencem a todo $U \in U N I V$; somas diretas e produtos tensorias de famílias $U$-pequenas de $\mathcal{R}$-modulos $U$-pequenos são $U$-pequenos, onde $\mathcal{R}$ é um anel comutativo com unidade $U$-pequeno; completamentos de espaços métricos $U$-pequenos; via classes de equivalência de seqüências de Cauchy; são $U$-pequenos; compactificações de Stone-Čech de espaços $T_{3+1 / 2} U$-pequenos são $U$-pequenos; compactificações de Alexandroff de espaços $T_{2}$ localmente compactos $U$-pequenos são $U$-pequenos (para o espaço $\mathcal{X}=(X, \tau)$ podemos tomar, pela proposição (1i) e o axioma da regularidade, $\infty=\{X\})$; anéis de polinômios em $X \in U$ variáveis sobre anéis $U$-pequenos são $U$-pequenos; fechos algébricos de corpos $U$-pequenos são $U$-pequenos... . Assim, na matemática cotidiana, tudo se passa como se "vivêssemos" em um universo $U$ fixo e arbitrário. Contudo, fazendo fundamentos; algumas questoẽs são respondidas apenas quando consideramos condições adicionais sobre o universo de base. Algumas dessas surgirão adiante.

Agora, é natural considerarmos a:

Definição 2 : Sejam $U \in U N I V$ e $C$ categoria, eventualmente classe própria:

(i) Denotamos por $C_{\left.\right|_{U}}\left(C_{\left.\right|_{U}}\right)$ a subcategoria plena de $C$ de todos os objetos pertencentes a $U$ (isomorfos a algum objeto pertencente a $U$ ).

Exemplos: $U-S E T=\left.S E T\right|_{U=}, U-C A T=\left.C A T\right|_{U=}, \operatorname{obj}\left(\left.S E T\right|_{U} \cong\right)=\{x \in V: \# x<$ $\# U\}$.

(ii) $C$ é uma categoria em $U \Longleftrightarrow C \in \operatorname{obj}\left(\left.C A T\right|_{U=}\right)$.

Exemplos: Se $W \in U N I V$ e $W \in U$ então $W-S E T, W-G R, W-C A T$, ... são categorias em $U$; se $A$ e $B$ são categorias em $U$ então $B^{A}(=[A, B])$ é uma categoria em $U$.

(iii) $C$ é uma categoria praticamente em $U \Longleftrightarrow C \in \operatorname{obj}\left(\left.C A T\right|_{U} \cong\right)$.

Exemplos: Se $A$ e $B$ são categorias praticamente em $U$ então $B^{A}$ é categoria praticamente em $U$.

(iv) $C$ é uma categoria essencialmente em $U \Longleftrightarrow \epsilon s q(C)$ é uma categoria praticamente em $U$, onde $\epsilon s q(C)$ é o (único a menos de isomorfismo) esqueleto de $C$.

Exemplos: Para cada cardinal $\lambda<\# U$ sejam $S E T_{<\lambda}$; a subcategoria plena de $S E T$ dos conjuntos de cardinalidade $<\lambda, G R<\lambda$, a subcategoria plena de $G R$ dos grupos $<\lambda$-gerados , ... 
(v) $C$ é uma categoria localmente em $U \Longleftrightarrow \forall a, b \in \operatorname{obj}(C)(C(a, b) \in U)$.

Exemplos: $U-S E T, U-G R, U-T O P, U-C A T$.... são categorias localmente em $U$.

(vi) $C$ é uma categoria praticamente localmente em $U \Longleftrightarrow \forall a, b \in$ obj $(C)(\# C(a, b) \in$ $U)$.

Exemplos: Se $A$ é categoria (praticamente) em $U$ e $B$ é categoria (praticamente) localmente em $U$ então $B^{A}$ é categoria praticamente localmente em $U$.

Observação 5 : Relativizamos acima as noções de categorias pequena e localmente pequena. Temos, para categorias praticamente localmente em $U \in U N I V$; lemas e mergulhos de Yoneda relativizados a $U-S E T$ e que são compatíveis:

(i) Dada $C$ categoria praticamente localmente em $U$ o funtor de Yoneda $Y_{C}: C \rightarrow$ $\left[C^{\star}, S E T\right]$ se fatora, a menos de isomorfismo natural, através de $i_{U}:\left[C^{\star}, U-S E T\right] \hookrightarrow$ $\left[C^{\star}: S E T\right]$, i.e. $Y_{C} \cong i_{U} \circ Y_{C}^{U}$ e o funtor $Y_{C}^{U}: C \rightarrow\left[C^{\star}: U-S E T\right]$ tem a "propriedade de Yoneda relativizada".

(ii) Se $C$ está praticamente localmente em $U$ e em $U^{\prime}$ e $U \subseteq U^{\prime}$ então $i_{U U^{\prime}}:\left[C^{\star}, U-\right.$ $S E T] \hookrightarrow\left[C^{\star}, U^{\prime}-S E T\right], Y_{C}^{U}: C \rightarrow\left[C^{*}: U-S E T\right]: Y_{C}^{U^{\prime}}: C \rightarrow\left[C^{\star}, U^{\prime}-S E T\right]$ e $Y_{C}^{U^{\prime}} \cong$ $i_{U U^{\prime}} \circ Y_{C}^{U}$.

(iii) De modo geral todas as definições ; construções e teoremas da teoria das categorias podem ser relativizados e, freqüentemente, bastam três níveis $U_{0} \in U_{1} \in U_{2} \in V$ ("conjuntos", "classes" e "metaclasses") , arbitrários em princípio, que são "representativos" e onde podemos formular as definições , construções e teoremas. Assim, eventualmente não especificaremos os estágios em que estamos ou se estamos efetivamente tratando de classes próprias, no máximo nos limitando a observar que uma dada definição : construção ou teorema pode, de fato, ser relativizado.

\subsection{Fatos básicos sobre categorias}

Colocamos definições e alguns resultados necessários ao desenvolvimento do trabalho. As referências centrais são [159] ; [186] ; para o ítem (1.2.4) sugerimos também [175].

\subsubsection{Grafos e categorias}

A existência deste ítem a esta altura, quando assumimos familiaridade "metateórica" de grafos e categorias, se justifica porque assim antecipamos exemplos importantes do nosso objeto de estudo : estruturas apresentadas "diagramaticamente" ; como será precisado no cap.3 e item 6.2

Um grafo $G$ é uma quadra ordenada $G=\left(G_{0}, G_{1}, d, c\right)$ tal que $G_{0}(=o b j(G))$ e $G_{1}$ $(=\operatorname{mor}(G))$ são classes e $d\left(=\operatorname{dom}_{G}\right)$ e $c\left(=\operatorname{codom}_{G}\right)$ são funções tais que : 
[diagrama: grafo]

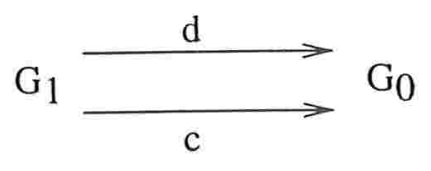

Sejam $G$, $G^{\prime}$ grafos. Um homomorfismo $F$, de domínio $G$ e codomínio $G^{\prime}$ é um par ordenado $F=\left(F_{0}, F_{1}\right)$ de funções tal que os diagramas (de classes e funções ) comutam:

[diagrama: homomorfismo de grafos]

(1) $\forall f \in G_{1}\left(d^{\prime} \circ F_{1}(f)=F_{0} \circ d(f)\right)$

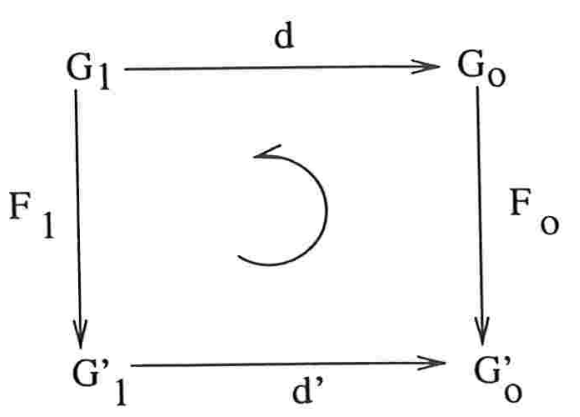

$(2) \forall f \in G_{1}\left(c^{\prime} \circ F_{1}(f)=F_{0} \circ c(f)\right)$

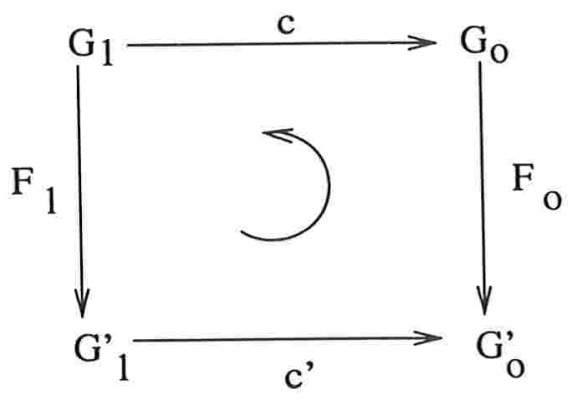

Seja $G$ grafo, denotamos $\# G=\# G_{0}+\# G_{1}$ (cardinalidade de $G$ ). $G$ é um grafo pequeno $\Longleftrightarrow G \in V(\Longleftrightarrow \# G<\infty)$ e é $U$ - pequeno $\Longleftrightarrow G \in U \in U N I V . G R A(U-G R A)$ é a categoria dos grafos pequenos ( $U$-pequenos) e homomorfismos.

$C$ é uma categoria $\Longleftrightarrow C=\left(C_{0}, C_{1}, d, c, i, k\right)$ onde $\left(C_{0}, C_{1}, d, c\right)\left(=\mathcal{E}_{0}(C)\right)$ é um grafo e $i\left(=i d_{C}\right)$ e $k\left(=\right.$ comp $\left._{C}\right)$ são funções : $i: C_{0} \rightarrow C_{1} a \mapsto 1_{a}$ e $k: C_{2} \rightarrow C_{1}(f: g) \mapsto g \circ f$ : onde definimos as seguintes classes e funções :

. $C_{2}=\left\{(f, g) \in C_{1} \times C_{1}: d(g)=c(f)\right\}$.

. $C_{3}=\left\{(f, g ; h) \in C_{1} \times C_{1} \times C_{1}:(f, g) \in C_{2} \wedge(g ; h) \in C_{2}\right\}$.

- $p_{1}, p_{2}: C_{2} \rightarrow C_{1}$.

. $q_{1}: q_{2}, q_{3}: C_{3} \rightarrow C_{1}$.

- $\left(q_{1}, q_{2}\right),\left(q_{2}: q_{3}\right),\left(q_{1}, k\right),\left(k, q_{3}\right): C_{3} \rightarrow C_{2}$.

. $\left(i \circ d, 1_{C_{1}}\right),\left(1_{C_{1}}, i \circ c\right): C_{1} \rightarrow C_{2}$. 
Tais que:

$C_{2}$ e $C_{3}$ são dados como "limites projetivos canônicos" com (algumas) projeções dadas por $p_{1}, p_{2}$ e $q_{1}: q_{2}, q_{3}$.

. As flechas restantes são definidas utilizando-se as propriedades universais de $C_{2}$ e $C_{3}$. [diagrama: categoria]

(1)def. $C_{2}$

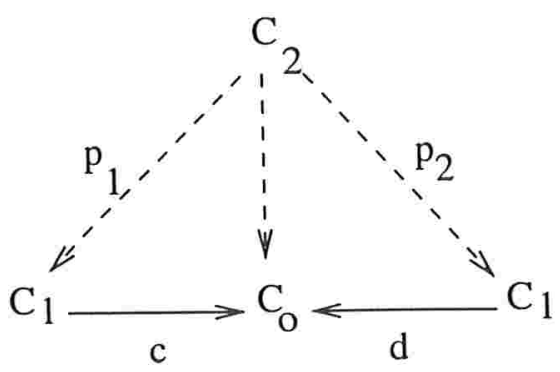

(2)def. $C_{3}$

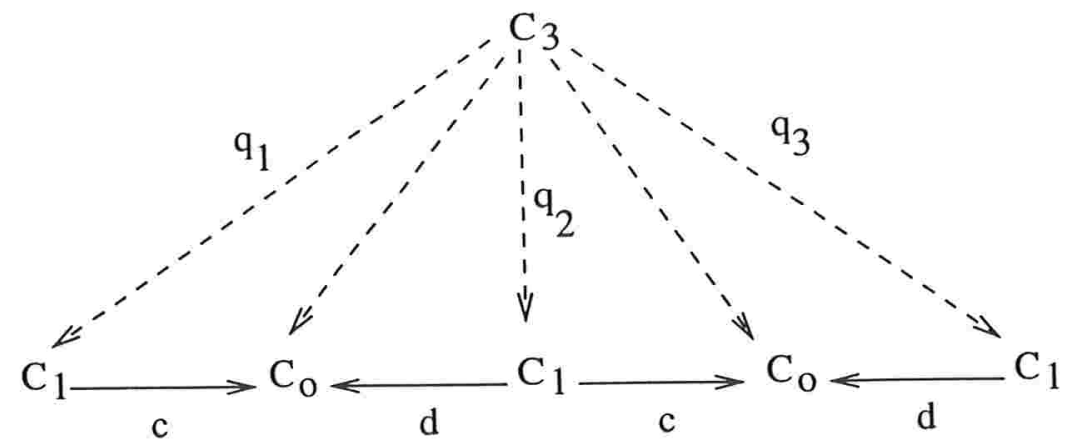

(3)def. $\left(q_{1}, q_{2}\right)$

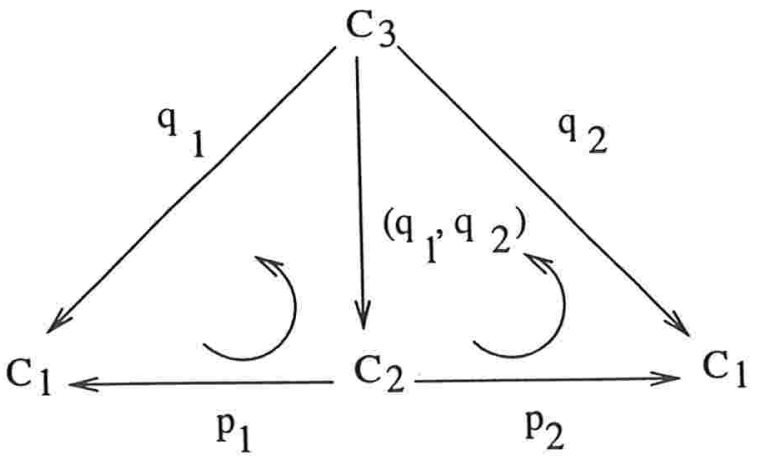

(4)def. $\left(q_{2}, q_{3}\right)$ 


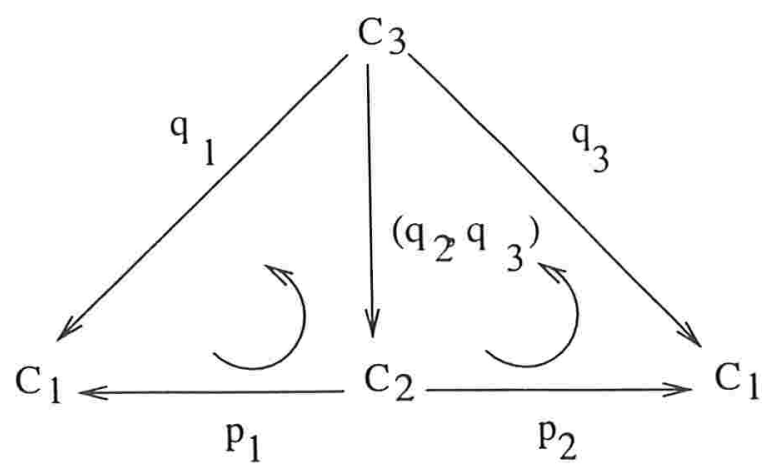

(5)def. $\left(q_{1}, k\right)$

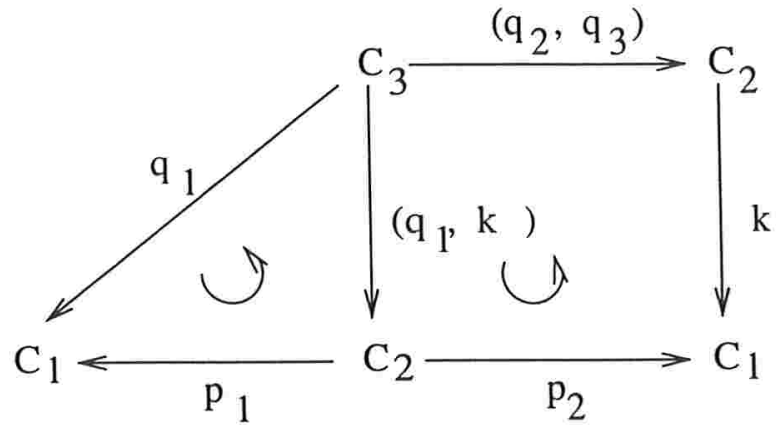

(6)def. $\left(k, q_{3}\right)$

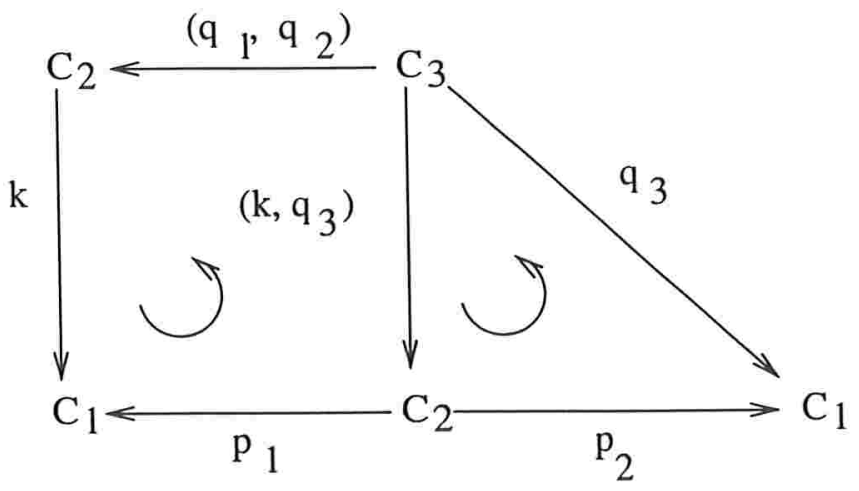

(7)def. $\left(i \circ d, 1_{C_{1}}\right)$ 


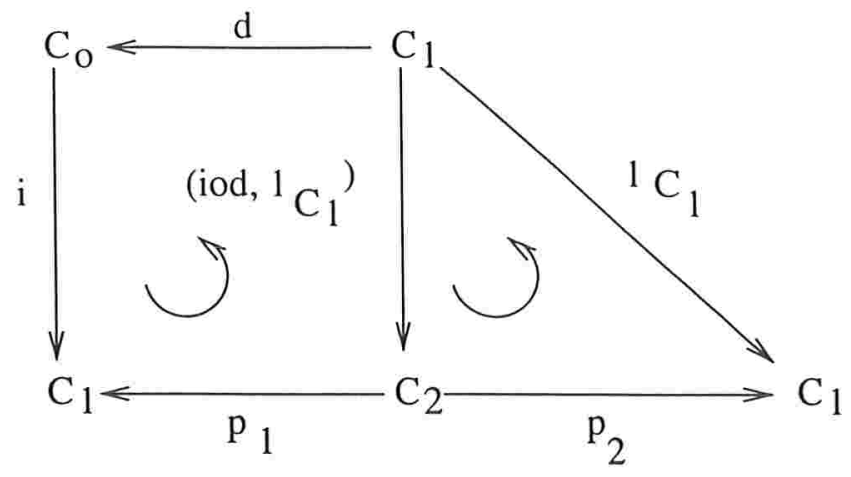

(8)def. $\left(1_{C_{1}} ; i \circ c\right)$

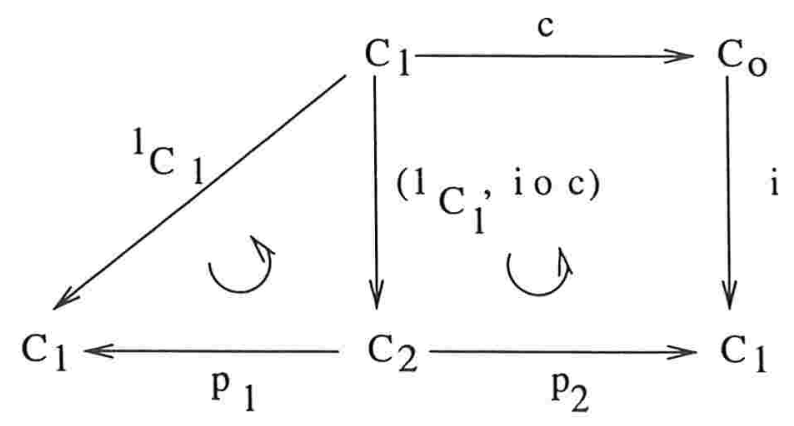

satisfazendo:

(9) $\forall a \in C_{0}\left(d\left(1_{a}\right)=a\right)$

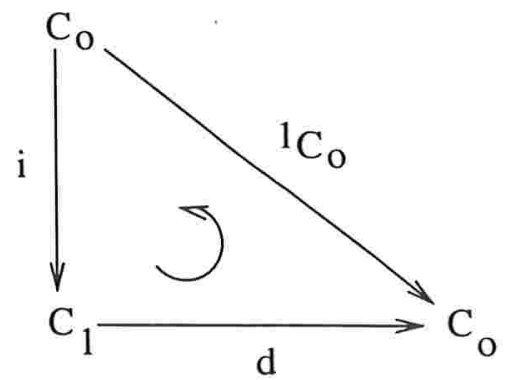

(10) $\forall a \in C_{0}\left(c\left(1_{a}\right)=a\right)$

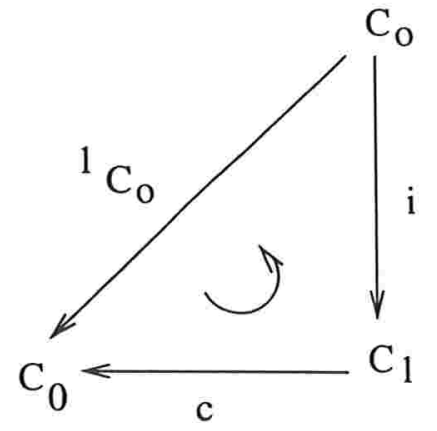

$(11) \forall(f, g) \in C_{2}(d(g \circ f)=d(f))$ 


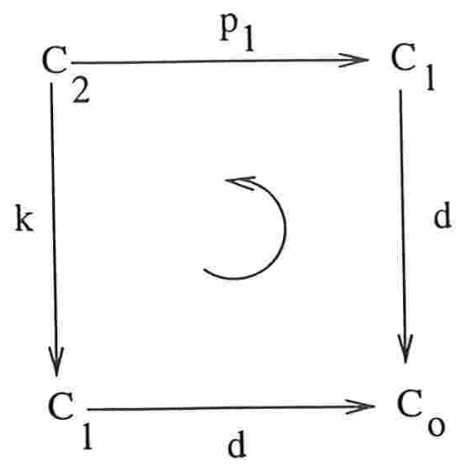

$(12) \forall(f: g) \in C_{2}(c(g \circ f)=c(g))$

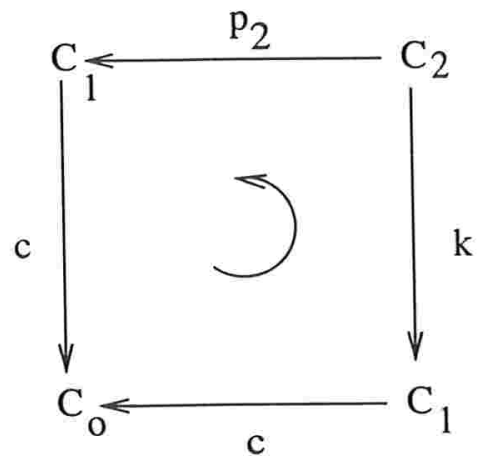

(13) $\forall f \in C_{1}\left(f \circ 1_{d(f)}=f\right)$

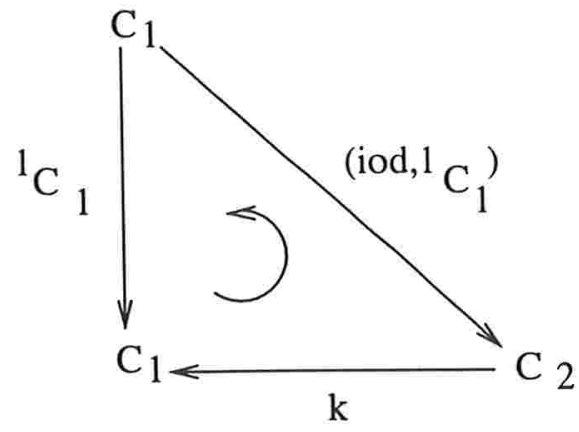

$(14) \forall g \in C_{1}\left(1_{c(g)} \circ g=g\right)$

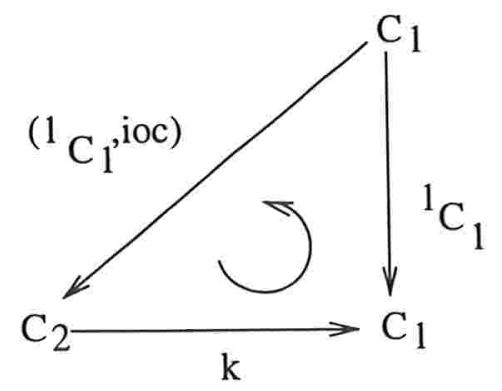

$(15) \forall(f: g, h) \in C_{3}(h \circ(g \circ f)=(h \circ g) \circ f)$ 


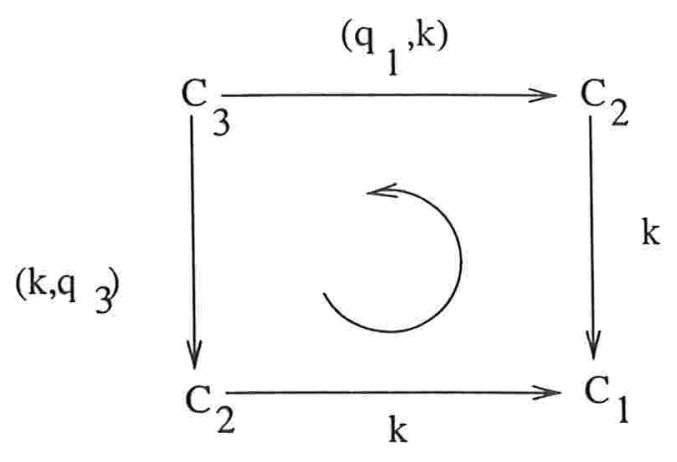

Sejam $C, C^{\prime}$ categorias. Um funtor $F$ de domínio $C$ e codomínio $C^{\prime}$ é um par ordenado $F=\left(F_{0}, F_{1}\right)$ tal que $F\left(=\mathcal{E}_{1}(F)\right)$ é um homomorfismo de domínio $\mathcal{E}_{0}(C)$ codomínio $\mathcal{E}_{0}\left(C^{\prime}\right)$ e tal que os diagramas comutam:

[diagrama: funtor]

(3) $\forall a \in C_{0}\left(F_{1}\left(1_{a}\right)=1_{F_{0}(a)}^{\prime}\right)$

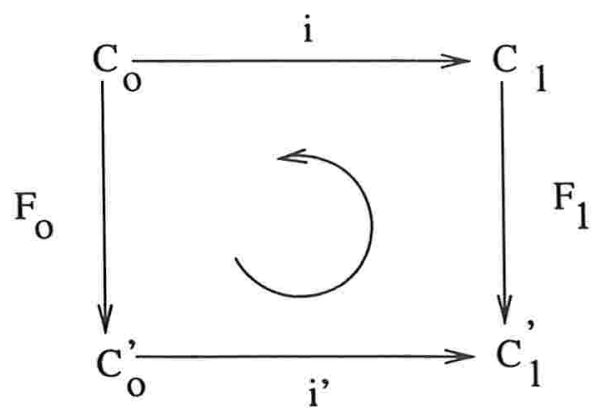

$(4) \forall(f ; g) \in C_{2}\left(F_{1}(g \circ f)=F_{1}(g) \circ^{\prime} F_{1}(f)\right)$

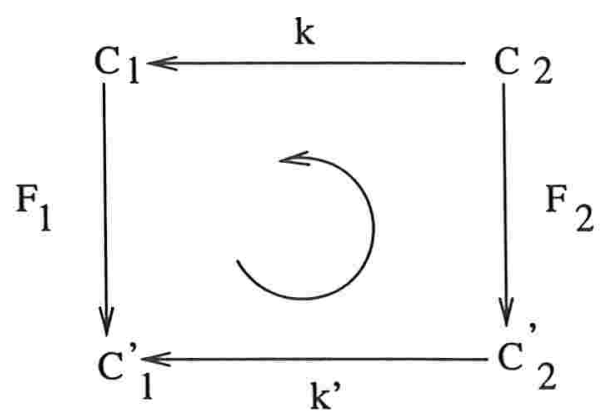

onde $F_{2}=\left(F_{1} \times F_{1}\right)$ I: $C_{2} \rightarrow C_{2}^{\prime}$; isto é:

(5)def. $F_{2}$ 


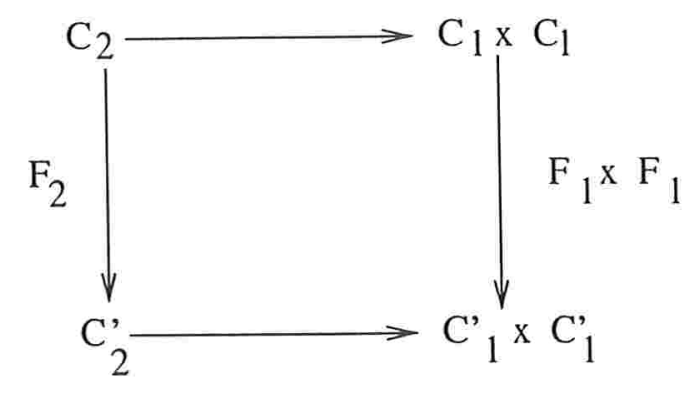

Seja $C$ categoria, denotamos $\# C=\# C_{0}+\# C_{1}\left(=\# \mathcal{E}_{0}(C)\right)$. Notemos que $\# C_{0} \leq \# C_{1}$ e $C$ é categoria pequena ( $U$-pequena) $\Longleftrightarrow \mathcal{E}_{0}(C)$ é grafo pequeno ( $U$-pequeno).

\subsubsection{Categorias livres, quocientes e diagramas comutativos}

Dado $n \in \omega$ denotamos por $\vec{n}$ o grafo pequeno (e que pertence a todo universo $U$ !) tal que $\operatorname{obj}(\vec{n})=n \in \omega$ e $\forall i, j \in n \vec{n}(i ; j)=\{(i, j)\}$ se $j=i+1$ e $\vec{n}(i, j)=\emptyset$ se $j \neq i+1$. Para cada grafo $G \in V(\in U)$ e para cada $n \in \omega$ definimos $L_{n}(G)=G R A(n \overrightarrow{+1}, G) \in V(\in U)$, temos $L_{n}(G) \cap L_{m}(G) \neq \emptyset \Rightarrow n=m$. Portanto, se para cada $a, b \in o b j(G)$, denotamos $L_{n}(G)(a, b)=\left\{\Gamma \in L_{n}(G): \Gamma(0)=a \wedge \Gamma(n)=b\right\}$ temos $L_{n}(a, b) \cap L_{m}(c, d) \neq \emptyset \Rightarrow$ $(n=m \wedge(a, b)=(c, d))$ e $\left\{L_{n}(a, b): n \in \omega \wedge a, b \in G_{0}\right\} \in V(\in U)$. Definindo $\forall a, b \in G_{0}\left(L(G)(a, b)=\cup_{n \in \omega} L_{n}(G)(a, b)\right), \forall n \in \omega\left(L_{n}(G)=\cup_{a, b \in G_{0}} L_{n}(G)(a, b)\right)$ e $L(G)=\cup\left\{L_{n}(a, b): n \in \omega \wedge a, b \in G_{0}\right\}$, notamos que:

(i) $G_{0} \cong L_{0}(G)$ e $G_{1} \cong L_{1}(G)$.

(ii) Temos funções evidentes :

. $i: G_{0} \rightarrow L(G)$.

. $\forall n, m \in \omega \forall a, b, c \in G_{0}\left(k_{(n, m)}(a, b, c): L_{n}(G)(a, b) \times L_{m}(G)(b, c) \rightarrow L_{n+m}(G)(a, c)\right)$.

(iii) Todas as reuniões acima são disjuntas.

(iv) As aplicações acima determinam uma categoria $\mathcal{L}(G)$ tal que $\operatorname{obj}(\mathcal{L}(G))=o b j(G)$ e $\operatorname{mor}(\mathcal{L}(G))=L(G)$ e um homomorfismo universal $\eta(G): G \rightarrow \mathcal{E}(\mathcal{L}(G))$, isto é: $\forall C$ categoria $\forall F: G \rightarrow \mathcal{E}(C) \exists ! F^{\prime}: \mathcal{L}(G) \rightarrow C\left(F=\mathcal{E}\left(F^{\prime}\right) \circ \eta(G)\right)$.

(v) $G \in V(\in U) \Rightarrow \mathcal{L}(G) \in V(\in U)$ e $\# \mathcal{L}(G)+\omega=\# G+\omega$.

(vi) Para toda categoria $C$ e todo grafo $G \in V$, as categorias "exponenciais" $C^{G}$ e $C^{\mathcal{L}(G)}$ são isomorfas.

(vii) Sejam $C \in(C A T)_{0}$ e $X=\left(X_{0}, X_{1}\right)$ onde $X_{0} \subseteq C_{0}: X_{1} \subseteq C_{1}$. Temos que $\mathcal{G}(X)$ : o subgrafo de $\mathcal{E}(C)$ gerado por $X$, é tal que $\# X \leq \# \mathcal{G}(X) \leq \# X+2$. \#X $; X_{1} \mathcal{C}(X)$ : a subcategoria de $C$ gerada por $X$, é tal que $\# X \leq \# \mathcal{G}(X) \leq \# \mathcal{C}(X) \leq \# \mathcal{L}(\mathcal{G}(X))$ e $\omega+\# X=\omega+\# \mathcal{G}(X)=\omega+\# \mathcal{C}(X)$.

Uma relação $R$ numa categoria $C$ é uma aplicação $R:(a, b) \in C_{0} \times C_{0} \mapsto R_{a b} \subseteq$ $C(a, b) \times C(a, b) \in \mathcal{P}\left(C_{1} \times C_{1}\right)$. A relação $R$ é uma congruência $\Longleftrightarrow \forall a, b \in C_{0}\left(R_{a b}\right.$ 
é uma relação de equivalência em $C(a, b)) \wedge \forall a, b, c . d \in C_{0} \forall g, g^{\prime} \in C(b, c) \forall f \in C(a, b)$ $\forall h \in C(c, d)\left(\left(g: g^{\prime}\right) \in R_{b c} \Rightarrow\left(h \circ g \circ f . h \circ g^{\prime} \circ f\right) \in R_{a d}\right)$. Temos que:

(i) A "intersecção " de congruências é congruência e. portanto, a classe das congruências numa categoria, ordenada por "inclusão", é um reticulado (grande) completo.

(ii) Se $R$ é uma congruência em $C$ podemos definir a categoria quociente $C / R$ e o funtor projeção canônica $\Pi_{R}: C \rightarrow C / R$.

(iii) $C \in V(\in U) \Rightarrow C / R \in V(\in U)$.

Dado $F: C \rightarrow D$ funtor, seja $k \epsilon r(F)$ a relaçào em $C$ tal que $\forall a, b \in C_{0}\left(k \in r(F)_{a b}=\right.$ $\{(f, g) \in C(a, b) \times C(a, b): F(f)=F(g)\})$; então:

(i) $k \in r(F)$ é uma congruência em $C$.

(ii) $F: C \rightarrow D$ se fatora como a composição de um funtor localmente sobrejetor (pleno) e um funtor localmente injetor (fiel).

(iii) Se $R$ é congruência em $C$ então $R=k \epsilon r\left(\Pi_{R}\right)$.

(iv) Temos o "teorema do homomorfismo" : dada $R$, relação em $C$, então todo funtor $F: C \rightarrow D$ tal que $R \leq \operatorname{ker}(F)$ se fatora univocamente por $\Pi_{\angle B>}: C \rightarrow C /<R>$ onde $<R>$ é a congruência em $C$ gerada por $R$.

(v) Toda categoria $C$ é determinada por geradores e relações : o funtor "composição em $C$ " $\varepsilon(C): \mathcal{L}(\mathcal{E}(C)) \rightarrow C$ é a identidade nos objetos; localmente sobrejetor e $C \cong$ $\mathcal{L}(\mathcal{E}(C)) / k \in r(\varepsilon(C))$.

(vi) Para toda categoria $C \in V$, toda relação $R$ em $C$ e toda categoria $A$; as categorias $A^{(C, R)}$ e $A^{C K R>}$ são isomorfas, onde $A^{(C, R)}$ é a subcategoria plena de $A^{C}$ tal que $\operatorname{obj}\left(A^{(C, R)}\right)=$ $\{F: C \rightarrow A: R \leq k \in r(F)\}$.

Dados $I$ grafo pequeno e $C$ categoria ( $G$ grafo), os homomorfismos $F$ de domínio $I$ e codomínio $\mathcal{E}(C)(G)$ são denominados diagramas de tipo $I$ em $C(G)^{1} . F: I \rightarrow \mathcal{E}(C)$ é um diagrama comutativo $\Longleftrightarrow \forall i, j \in \operatorname{obj}(I) \forall \Gamma, \Gamma^{\prime} \in \mathcal{L}(I)(i, j)\left(F^{\prime}(\Gamma)=F^{\prime}\left(\Gamma^{\prime}\right)\right)$ $\left.\Longleftrightarrow k \in r\left(F^{\prime}\right)=\mathrm{\top}_{\text {cong }(\mathcal{L}(I))}\right)$ (= máximo do reticulado das congruências em $\mathcal{L}(I)$ ).

Dados $G$ grafo $(G \in V ; G \in U)$ e $D$ classe de diagramas de tipo pequeno $(D \in V ; D \in U)$ seja $R(D)$ a relação em $\mathcal{L}(G)$ tal que $\forall g, h \in \operatorname{obj}(G)\left(R(D)_{g h}=\left\{\left(\Gamma, \Gamma^{\prime}\right) \in(\mathcal{L}(G)(g, h))^{2}\right.\right.$ : $\exists d: I \rightarrow G \in D \exists i, j \in \operatorname{obj}(I) \exists\left(\Psi, \Psi^{\prime}\right) \in(\mathcal{L}(I)(i, j))^{2}\left((g ; h)=(d(i), d(j)) \wedge\left(\Gamma, \Gamma^{\prime}\right)=\right.$ $\left.\left.\left(\mathcal{L}(d)(\Psi), \mathcal{L}(d)\left(\Psi^{\prime}\right)\right)\right)\right\}$ ) : então a categoria $(\in V: \in U)(G: D)=\mathcal{L}(G) /\langle R(D)>$ e o diagrama $\nu(G, D)=\mathcal{E}\left(\Pi_{<R(D)>}\right) \circ \eta(G): G \rightarrow \mathcal{E}((G: D))$ são tais que:

(i) $\forall d \in D\left(\nu(G, D) \circ d\right.$ é diagrama comutativo ) ( notar que $(\eta(G) \circ d)^{\prime}=\mathcal{L}(d): \mathcal{L}(I) \rightarrow$ $\mathcal{L}(G))$

(ii) $\forall C$ categoria $(\in V: \in U) \forall M: G \rightarrow \mathcal{E}(C)(\forall d \in D$ (Mod é diagrama comutativo) $\Rightarrow$ $\exists ! \bar{M}:(G: D) \rightarrow C(M=\mathcal{E}(\bar{M}) \circ \nu(G: D))\left(\right.$ notar que $\left.(M \circ d)^{\prime}=M^{\prime} \circ \mathcal{L}(d): \mathcal{L}(I) \rightarrow C\right)$.

\footnotetext{
${ }^{1}$ Convém definir diagramas em categorias como um par homomorfismo-categoria pois categorias distintas podem ter mesmo grafo subjacente.
} 
(iii) $\#(G: D) \leq \omega+\# G$.

Destacamos que para todo $G$ grafo pequeno $(G \in V: G \in U)$ : toda classe $D$ de diagramas de tipo pequeno em $G(D \in V ; D \in U)$ e toda categoria $C(C \in V: C \in U)$ temos $C^{(G ; D) \cong}$ $C^{(G: D)}$ onde $C^{(G, D)}$ é a subcategoria plena de $C^{G}(\in V: \in U)$ tal que obj $\left(C^{(G, D)}\right)=\{M$ : $G \rightarrow \mathcal{E}(C): \forall d \in D(M \circ d$ é diagrama comutativo $)\}$.

\subsubsection{Cones}

Seja $C$ categoria; um objeto inicial $a \in o b j(C)$ é estrito $\Longleftrightarrow \forall c \in o b j(C)(c \neq a \Rightarrow$ $C(c, a)=\emptyset)$. Temos que:

(i) Exemplo: $\emptyset \in \operatorname{obj}(S E T)(\emptyset \in o b j(U-S E T))$.

(ii) Se uma categoria possui objeto inicial estrito então este é único, e não apenas único a menos de isomorfismo (notação : $-\infty$ ).

(iii) Toda categoria pequena $C$ pode ser imersa numa categoria pequena com objeto inicial estrito $C^{-}$de modo que se $C$ está praticamente em $U$ então $C^{-} \in \operatorname{obj}(U-C A T)$ :

Para cada $C$ seja (axioma da escolha global!) $F: C \rightarrow C^{\prime}$ isomorfismo, $\operatorname{com} C^{\prime} \in \operatorname{obj}(U-$ $C A T)$ e onde supomos $\operatorname{mor}\left(C^{\prime}\right) \cap\left\{\left(\operatorname{obj}\left(C^{\prime}\right), c^{\prime}\right): c^{\prime} \in \operatorname{obj}\left(C^{\prime}\right)\right\}=\emptyset$ e $\left(\operatorname{obj}\left(C^{\prime}\right): \operatorname{obj}\left(C^{\prime}\right)\right) \notin$ $\operatorname{mor}\left(C^{\prime}\right)$. Definimos:

(i) $\operatorname{obj}\left(C^{-}\right)=\operatorname{obj}\left(C^{\prime}\right) \cup\{-\infty\}$ onde $-\infty=\operatorname{obj}\left(C^{\prime}\right)$.

(ii) $C^{-}(-\infty,-\infty)=\{(-\infty,-\infty)\}$.

(iii) $\forall c \in \operatorname{obj}(C)\left(C^{-}(-\infty, F(c))=\{(-\infty, F(c))\} \wedge C^{-}(F(c) ;-\infty)=\emptyset\right)$.

(iv) Definimos de modo natural as funções $d, c, i, k$ e o funtor $J: C: C^{-}$.

Temos que:

(i) $\# C_{0}^{-}=\# C_{0}+1$ e $\# C_{1}^{-}=\# C_{1}+\# C_{0}+1$.

(ii) $J: C \rightarrow C^{-}$tem a seguinte propriedade: $\forall A$ categoria com objeto inicial $\forall F: C \rightarrow A$ funtor $\exists F^{\prime}: C^{-} \rightarrow A$ funtor que preserva objetos iniciais tal que $F^{\prime} \circ J \cong F$.

Temos ainda:

(i) No ítem (1.3.3) observaremos que $J: C \rightarrow C^{-}$é $\mathcal{I}$-ind completamento forte e fraco de $C \operatorname{com} \mathcal{I}=\{\emptyset\}$.

(ii) Se $A$ possui um único objeto inicial então para todo $F: C \rightarrow A F^{\prime}$ é único e $F^{\prime} \circ J=F$

(iii) $\mathrm{O}$ funtor de inclusão $I: C A T_{\text {obj.ini.єstr. }} \hookrightarrow C A T$ tem adjunto à esquerda $(-)^{-}$: onde

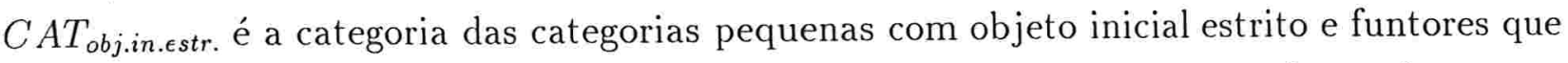
preservam objetos iniciais (notemos que estes funtores se restringem aos universos).

Seja $G$ grafo; $a \in o b j(G)$ é um (único!) objeto inicial estrito $\Longleftrightarrow G(a, a)=\emptyset \wedge$ $\forall g \in \operatorname{obj}(G)(g \neq a \Rightarrow(\# G(a, g)=1 \wedge G(g, a)=\emptyset))$. Assim para cada grafo pequeno $G$ construimos, analogamente às categorias, um homorfismo de imersão $J: G \hookrightarrow G^{-}$, onde $G^{-}$ é um grafo pequeno com (único) objeto inicial estrito, tal que: 
(i) $\# \operatorname{obj}\left(G^{-}\right)=\# \operatorname{obj}(G)+1$ e \#mor $\left(G^{-}\right)=\# \operatorname{mor}(G)+\# \operatorname{obj}(G)$.

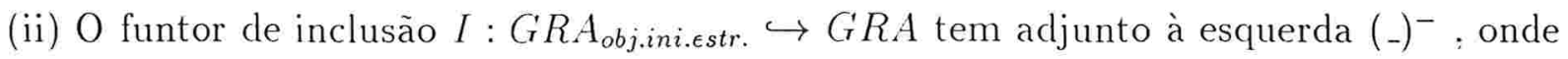

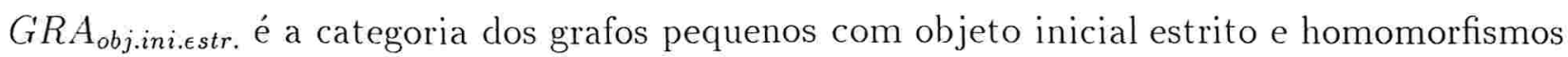
que preservam objetos iniciais estritos (esta adjunção se restringe a cada universo $U$ ).

Dualmente definimos, para categorias (grafos), a noção de objeto final estrito $(+\infty)$ e construimos para categorias (grafos) pequenos $C(G)$ funtores (homomorfismos) de imersão $J: C \rightarrow C^{+}\left(J: G \smile G^{+}\right)$em categorias (grafos) pequenos com objeto final estrito $C^{+}\left(G^{+}\right)$ com propriedades universais duais e que se restringem aos universos.

Fixamos, pelo axioma da escolha global, imersões $J$ determinando adjuntos à esquer-

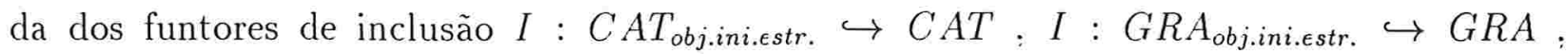

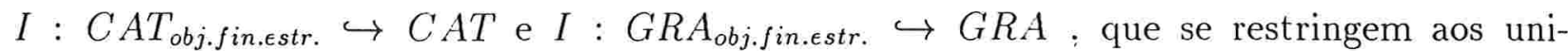
versos. Denotamos; para $C \in \operatorname{obj}(C A T)(G \in \operatorname{obj}(G R A)), C^{-}(-\infty, J(c))=\{\pi(c)\}$ e $C^{+}(J(c),+\infty)=\{\iota(c)\}\left(G^{-}(-\infty, J(g))=\{\pi(g)\}\right.$ e $\left.G^{+}(J(g),+\infty)=\{\iota(g)\}\right)$.

Notamos que:

(i) $C$ categoria $\Rightarrow\left(C^{ \pm}\right)^{\star} \cong\left(C^{\star}\right)^{\mp}$.

(ii) $G$ grafo $\Rightarrow\left(G^{ \pm}\right)^{\star} \cong\left(G^{\star}\right)^{\mp}$.

(iii) $G$ grafo não discreto (i.e. $\operatorname{mor}(G) \neq \emptyset) \Longleftrightarrow(\mathcal{L}(G))^{ \pm} ¥ \mathcal{L}\left(G^{ \pm}\right)$.

Dados $I$ grafo pequeno e $C$ categoria ( $G$ grafo), os homomorfismos de domínio $I^{-}$e codomínio $\mathcal{E}(C)(G)$ são denominados cones projetivos de tipo $I$ em $C(G)^{2}$. Dados $G$ grafo e $p: I^{-} \rightarrow G$ cone projetivo então base $(p)=p \circ J: I \rightarrow G$ e vértict $(p)=p(-\infty)$ . $p: I^{-} \rightarrow \mathcal{E}(C)$ é um cone projetivo comutativo $\Longleftrightarrow \forall i, j \in \operatorname{obj}(I) \forall u \in I(i, j)$ ( $p(u) \circ p(\pi(i))=p(\pi(j)))$.

Claramente temos bijeções :

(i) $I \in \operatorname{obj}(G R A) \Rightarrow\left\{p: I^{-} \rightarrow \mathcal{E}(C): p\right.$ é comutativo $\} \cong\left\{q: \mathcal{L}(I)^{-} \rightarrow C\right\}$.

(ii) $I \in \operatorname{obj}(C A T) \Rightarrow\left\{q: I^{-} \rightarrow C\right\} \cong\left\{p: \mathcal{E}(I)^{-} \rightarrow \mathcal{E}(C): p\right.$ é comutativo $\}$.

(iii) $I \in \operatorname{obj}(G R A) \wedge H: I \rightarrow \mathcal{E}(C) \wedge c \in \operatorname{obj}(C) \Rightarrow\left\{p: I^{-} \rightarrow \mathcal{E}(C): p\right.$ é comutativo $\wedge$ $\operatorname{bas\epsilon }(p)=H \wedge \operatorname{vértic\epsilon }(p)=c\} \cong N A T\left(\Delta_{I}(c), H\right)$ onde $\Delta_{I}: C \rightarrow C^{I}$ é o funtor "diagonal".

(iv) $I \in \operatorname{obj}(C A T) \wedge F: I \rightarrow C \wedge c \in \operatorname{obj}(C) \Rightarrow\left\{q: I^{-} \rightarrow C: \operatorname{base}(q)=F \wedge \operatorname{vértice}(q)=\right.$ $c\} \cong N A T\left(\Delta_{I}(c), F\right)$.

(v) $I \in \operatorname{obj}(G R A) \wedge H: I \rightarrow \mathcal{E}(C) \wedge c \in \operatorname{obj}(C) \Rightarrow\left\{p: I^{-} \rightarrow \mathcal{E}(C): p\right.$ é comutativo $\wedge$ $\operatorname{base}(p)=H \wedge \operatorname{vértice}(p)=c\} \cong N A T\left(\Delta_{\mathcal{L}(I)}(c): F\right)$ onde $F=H^{\prime}: \mathcal{L}(I) \rightarrow C$.

(vi) $I \in \operatorname{obj}(C A T) \wedge F: I \rightarrow C \wedge c \in \operatorname{obj}(C) \Rightarrow\left\{q: I^{-} \rightarrow C: \operatorname{base}(q)=q \circ J=\right.$ $F \wedge$ vértice $(q)=q(-\infty)=c\} \cong N A T\left(\Delta_{\mathcal{E}(I)}(c), H\right)$ onde $H=\mathcal{E}(F): \mathcal{E}(I) \rightarrow \mathcal{E}(C)$.

Dados $I$ grafo pequeno, $C$ categoria definimos coneproj $(I . C)=C^{\left(I^{-}\right)}$e $\operatorname{con} \epsilon \operatorname{proj} \operatorname{com}(I, C)$ a subcategoria plena (e fechada por isomorfismos) de $\operatorname{con} \epsilon \operatorname{proj}(I, C)$ tal que $o b j(\operatorname{con} \epsilon \operatorname{proj} \operatorname{com}(I$,

\footnotetext{
${ }^{2}$ Para categorias convém considerarmos cones como um par homomorfismo-categoria, pois categorias distintas podem ter mesmo grafo subjacente.
} 
$C))=\left\{p: I^{-} \rightarrow \mathcal{E}(C): p\right.$ é comutativo $\}$ (coneprojcom $\left.(I, C) \cong C^{\left(\mathcal{L}(I)^{-}\right)}\right)$. Dado $H:$ $I \rightarrow \mathcal{E}(C)$ denotamos $H$ - coneprojcom a subcategoria de coneprojcom $(I, C) \operatorname{com} \operatorname{obj}(H-$ coneprojcom $)=\left\{p: I^{-} \rightarrow \mathcal{E}(C): p\right.$ é comutativo $\wedge$ base $\left.(p)=H\right\}$ e tal que $\forall p, p^{\prime} \in$ obj( $H$ - coneprojcom) ( $H-$ coneprojcom $\left(p, p^{\prime}\right)=\left\{\eta \in N A T\left(p, p^{\prime}\right): \eta J=1_{H}: H \rightarrow H\right\}$ ). A subcategoria plena, eventualmente vazia, de $H$-contprojcom dos seus objetos finais (limites projetivos) é denotada $H$ - coneprojlim : coneprojlim $(I, C)$ é a subcategoria plena (e fechada por isomorfismos) de conєprojcom $(I, C)$ dos cones projetivos que são limites para alguma base $H: I \rightarrow \mathcal{E}(C)$. Quando $C \in \operatorname{obj}(C A T)$ temos uma bijeção entre os cones projetivos limites de base $H$ e as representações do funtor $N A T\left(\Delta_{\mathcal{L}(I)}(-), H^{\prime}\right): C^{\star} \rightarrow S E T$ . Para $I$ categoria pequena e $C$ categoria definimos $\operatorname{coneproj} \operatorname{com}(I, C)=C^{\left(I^{-}\right)} ; \cdots$

Analogamente definimos, através do funtor (-) ${ }^{+}: G R A \rightarrow G R A_{\text {obj.fin.estr. }}\left(\left(_{-}\right)^{+}: C A T \rightarrow\right.$ $\left.C A T_{\text {obj.fin.estr. }}\right)$ : cones indutivos, cones indutivos comutativos e cones indutivos limites (cones indutivos comutativos e cones indutivos limites).

Temos assim várias definições alternativas de cones projetivos/indutivos comutativos e cones projetivos/indutivos limites. A noção ; mais geral, de cone proj./ind. sobre grafos. obtida através dos funtores $\left({ }^{\mp}\right)^{\mp}$, é necessária no capítulo 3 .

A esta seção acrescentamos os seguintes fatos:

(i) Dados $I$ grafo pequeno e $C$ categoria temos isomorfismo: $\operatorname{con} \epsilon \operatorname{proj}(I, C) \cong \operatorname{con} \epsilon \operatorname{ind}\left(I^{\star}, C^{\star}\right)$ que se restringe às subcategorias plenas dos cones comutativos e limites e às subcategorias dos cones, cones comutativos, cones limites com bases $H: I \rightarrow \mathcal{E}(C)$ e $H^{\star}: I^{\star} \rightarrow \mathcal{E}\left(C^{\star}\right)$.

(ii) Dado $F: I \rightarrow C$ funtor:

. NAT $\left(\Delta_{I}(c), F\right) \cong \underset{I}{\stackrel{\lim }{\longleftarrow}} C(c ; F(i))$ natural em $c \in\left(C^{\star}\right)_{0}$.

. $N A T\left(F, \Delta_{I}(c)\right) \cong{\stackrel{\lim }{I^{\star}}} C(F(i), c)$ natural em $c \in C_{0}$.

(iii) Em geral, dados $F: F^{\prime}: I \rightarrow C$ funtores:

$N A T\left(F, F^{\prime}\right) \cong \int_{I} C\left(F(-), F^{\prime}(-)\right) \cong \underbrace{}_{I^{\S}} C\left(F(-), F^{\prime}(-)\right)^{\S} \cong “ \underbrace{\lim }_{u \in \operatorname{mor}(C)} C\left(F(c(u)), F^{\prime}(d(u))\right) "$ ( $I^{\S}$ é a categoria (associada a uma pré-ordem) de subdivisão de $I$ ) .

(iv) Dado $J \in C A T(C, A)$ temos funtores $J^{\rightarrow}: A \rightarrow(C A T \rightarrow C)$ e $J^{\leftarrow}: A \rightarrow(C A T \rightarrow$ $C)^{\star}$ :

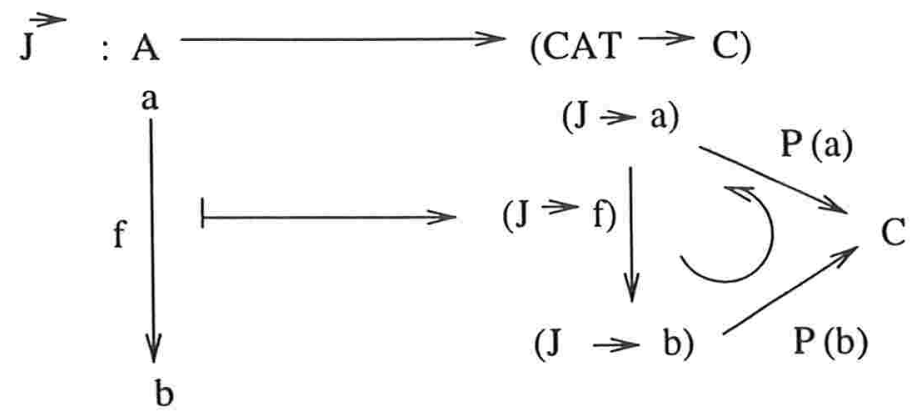




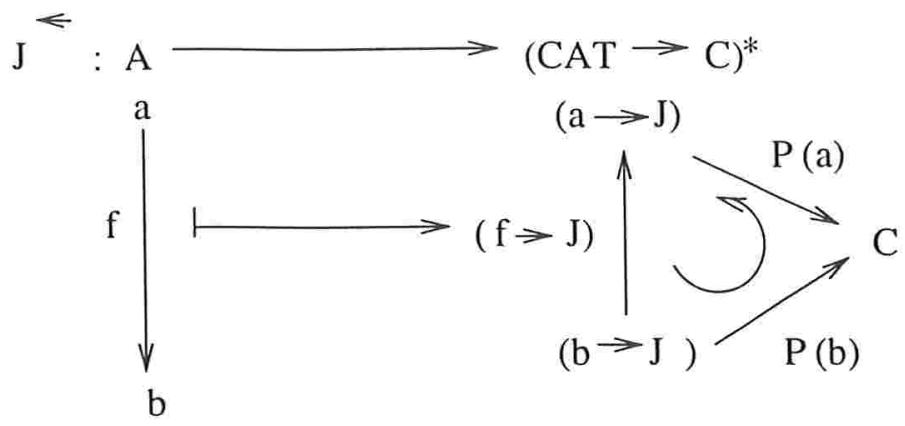

Temos que:

. $\forall F \in C A T(I, C)\left(N A T\left(J \circ F, \Delta_{I}(a)\right) \cong(C A T \rightarrow C)\left(F, J^{\rightarrow}(a)\right)\right)$ natural em $a \in A_{0}$.

- $\forall F \in C A T(I ; C)\left(N A T\left(\Delta_{I}(a), J \circ F\right) \cong(C A T \rightarrow C)^{\star}\left(J^{\leftarrow}(a), F\right)\right.$ natural em $a \in\left(A^{\star}\right)_{0}$

. Se $J \in U-C A T(C, A)$ podemos substituir $C A T \backsim U-C A T$.

- Se $C$ está praticamente localmente em $U \in U N I V$ podemos substituir $C A T \rightsquigarrow$ CATprat.loc.U .

(v) Dados $I, C \in \operatorname{obj}(C A T)$ temos funtores evidentes:

. $\operatorname{Proj}_{I}: C^{I} \rightarrow C A T\left(F \stackrel{\eta_{1}}{\rightarrow} F^{\prime}\right) \mapsto\left(F-\right.$ coneprojcom $\stackrel{\operatorname{Proj}_{\zeta}^{(\eta)}}{\rightarrow} F^{\prime}-$ coneprojcom $)$.

. Ind $d_{I}:\left(C^{I}\right)^{\star} \rightarrow C A T\left(F \stackrel{\eta_{1}}{\rightarrow} F^{\prime}\right) \mapsto\left(F^{\prime}-\right.$ contindcom $\stackrel{\text { Ind }_{I}(\eta)}{\rightarrow} F-$ contindcom $)$.

Se $\eta: F \stackrel{\cong}{\rightarrow} F^{\prime}$ então:

. $\left(\operatorname{Proj}_{I}(\eta)\right) \vdash: F-$ coneprojlim $\cong F^{\prime}-$ coneprojlim .

. $\left(\operatorname{Ind}_{I}(\eta)\right) \mid: F-$ coneindlim $\cong F^{\prime}$ - coneindlim .

Se $F \cong F^{\prime}: I \rightarrow C: F$ admite limite projetivo (indutivo) $\Longleftrightarrow F^{\prime}$ admite limite projetivo (indutivo) e estes são isomorfos quando existem .

(vi) Dada $C$ categoria temos funtores evidentes: transport $\epsilon^{-}$, transport $\epsilon^{+}:(C A T \rightarrow$ $C)^{\star} \rightarrow C A T$ que associam a cada "triângulo comutativo":

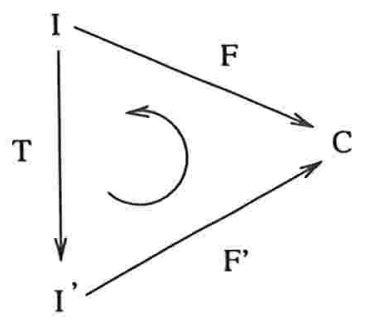

as flechas :

. _o $T^{-}: F^{\prime}-$ coneprojcom $\rightarrow F-$ coneprojcom .

. - o $T^{+}: F^{\prime}$ - coneindcom $\rightarrow F-$ coneindcom .

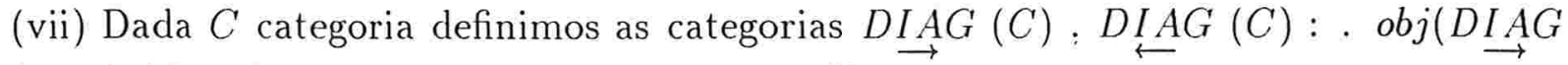
$(C))=\operatorname{obj}(D I A G(C))=I \in(\underset{\cup}{C A T})_{0} \operatorname{FUNT}(I, C)$. 
. $\forall F: I \rightarrow C: \forall F^{\prime}: I^{\prime} \rightarrow C\left(\underset{D I A G}{\longrightarrow}(C)\left(F, F^{\prime}\right)=\left\{(T . \eta): T \in F U N T\left(I, I^{\prime}\right) \wedge \eta \in\right.\right.$ $\left.N A T\left(F . F^{\prime} \circ T\right)\right\} \wedge D I A G(C)\left(F . F^{\prime}\right)=\left\{(T, \eta): T \in F U N T\left(I, I^{\prime}\right) \wedge \eta \in N A T\left(F^{\prime} \circ T, F\right)\right\}$ ).

$\stackrel{D I A G}{\longrightarrow}(C):$

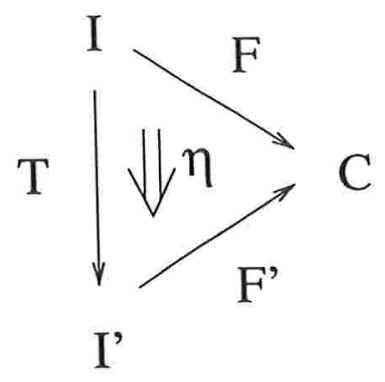

identidade $:\left(1_{I}, 1_{F}\right): F \rightarrow F$

composição: $F \stackrel{\left(T, \eta_{)}\right)}{\rightarrow} F^{\prime} \stackrel{\left(T^{\prime}, \eta^{\prime}\right)}{\rightarrow} F^{\prime \prime}=F^{\stackrel{\left(T^{\prime} \circ T, r^{\prime} T \cdot \eta^{\prime}\right)}{\rightarrow}} F^{\prime \prime}$

$D I A G(C)$ :

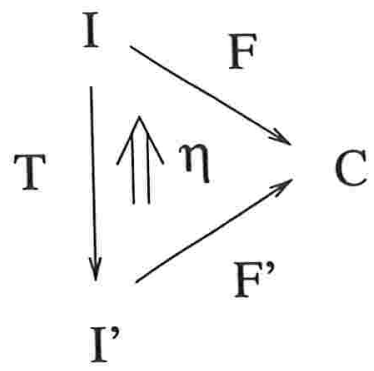

identidade : $\left(1_{I}, 1_{F}\right): F \rightarrow F$

composição : $F \stackrel{\left(T, r_{1}\right)}{\rightarrow} F^{\prime} \stackrel{\left(T^{\prime}, \eta^{\prime}\right)}{\rightarrow} F^{\prime \prime}=F^{\stackrel{\left(T^{\prime} \circ T, \eta_{r}, \eta_{1}^{\prime} T\right)}{\rightarrow}} F^{\prime \prime}$

(viii) Através da bijeção $C_{0} \cong F U N T(1, C)$ obtemos funtores $\underset{\rightarrow}{\triangle}(C): C \rightarrow D \underset{\operatorname{IA} G}{\rightarrow}(C)$ : $\underset{\leftarrow}{\Delta}(C): C^{\star} \rightarrow D I A G(C)$ tais que $\forall F: I \rightarrow C\left(F-\right.$ contindlim $\left.\cong \overrightarrow{p l} \in \operatorname{na}((F \rightarrow \Delta \overrightarrow{(C)}))_{\text {obj.ini. }}\right)$ $\stackrel{\leftarrow}{\wedge} F-$ coneprojlim $\cong$ plena $\left.\left((F \rightarrow \Delta(C))_{\text {obj.ini. }}\right)\right)$

(ix) Sejam $I, A, C$ categorias e $\stackrel{\leftarrow}{H}: I \rightarrow C, J: C \rightarrow A$ funtores:

. Dado $p \in(H-\text { coneprojlim })_{0}\left(J\right.$ preserva $\left.p \Longleftrightarrow J \circ p \in(J \circ H-\text { coneprojlim })_{0}\right)$. $J$ preserva limites projetivos para $H \Longleftrightarrow \forall p \in(H-\text { coneprojlim })_{0}(J$ preserva $p)$.

Obs.: $(H-\text { coneprojlim })_{0} \neq \emptyset \Rightarrow(J$ preserva limites projetivos para $H \Longleftrightarrow \exists p \in$ $(H-\text { coneprojlim })_{0}(J$ preserva $\left.p)\right)$.

Ex.: $C$ categoria localmente pequena (localmente em $U) \wedge c \in C_{0} \Rightarrow C(c ;-): C \rightarrow S E T$ $(C(c ;-): C \rightarrow U-S E T)$ preserva todos os limites projetivos.

(x) Sejam I, A, $C$ categorias e $H: I \rightarrow C, J: C \rightarrow A$ funtores:

. Dado $q \in(J \circ H-\text { coneprojlim })_{0}\left(J\right.$ cria $q \Longleftrightarrow \exists ! p \in(H-\text { coneprojcom })_{0}(q=J \circ p \wedge$ $\left.\left.p \in(H \text {-contprojlim })_{0}\right)\right) . J$ cria limites projetivos para $H \Longleftrightarrow \forall q \in(J \circ H \text {-contprojlim })_{0}$ 
( $J$ cria $q)$.

Ex.:

. $\forall U \in U N I V$ ( $i: U-S E T \hookrightarrow S E T$ cria limites proj./ind. canônicos $U$-pequenos ) (mas não todos os limites proj./ind. $U$-pequenos !).

. Se $J: C \rightarrow A$ preserva todos os limites projetivos (indutivos) $I$ - indexados então para cada $a \in A_{0} P(a):(a \rightarrow J) \rightarrow C(P(a):(J \rightarrow a) \rightarrow C)$ cria todos os limites projetivos (indutivos) $I$-indexados.

. Limites projetivos (indutivos) em categorias exponenciais são "pontuais":

Seja $B$ categoria pequena e $j: D\left(B_{0}\right) \rightarrow B$ a imersão da, única a menos de isomorfismo, categoria discreta com objetos $B_{0}$ em $B$, então para cada categoria $C$ o funtor $C^{j}: C^{B} \rightarrow$ $C^{D\left(B_{0}\right)}$ cria limites projetivos (indutivos) para todo tipo $I$. Notemos que $B_{0} \neq \emptyset \Rightarrow\left(C^{B}\right.$ $I-\operatorname{proj}(I-i n d)$ completa $\Longleftrightarrow C I-\operatorname{proj}(I-i n d)$ completa $)$; neste caso os $I-\operatorname{proj}$ $(I-i n d)$ limites em $C^{B}$ são pontuais, em particular, para cada funtor $F: A \rightarrow B$ o funtor $C^{F}: C^{B} \rightarrow C^{A}$ preserva $I$-limites projetivos (indutivos).

(xi) Definições e resultados semelhantes aos em (ix),(x) ocorrem quando consideramos $I \in \operatorname{obj}(G R A)$ e $H: I \rightarrow \mathcal{E}(C)$ homomorfismo:

. $J$ cria limites proj. (ind.) para $H \Rightarrow J$ preserva limites proj. (ind.) para $H$.

- Se $C, A$ são $I$-proj completas ( $I$-ind completas) então existem morfismos canônicos can : $J(\underset{I}{\lim } H) \rightarrow \underset{I}{\lim }(J \circ H)(\operatorname{can} \underset{I}{: \lim }(J \circ H) \rightarrow J(\underset{I}{\lim } H))$; neste caso $J$ preserva limites projetivos (indutivos) para $H \Longleftrightarrow$ os (essencialmente únicos) morfismos canônicos são isomorfismos.

\subsubsection{O funtor "componentes conexas" e funtores finais/iniciais}

Para cada grafo $G$ definimos $R(G)=\left\{(g, h) \in G_{0} \times G_{0}: G(g, h) \neq \emptyset\right\}$, então:

(i) Dada $C$ categoria $R(\mathcal{E}(C))$ é pré-ordem (reflexiva + transitiva) em $C_{0}$.

(ii) Dado $F: G \rightarrow G^{\prime}$ homomorfismo $\left(F_{0} \times F_{0}\right)_{*}(R(G)) \subseteq R\left(G^{\prime}\right)$.

Para cada grafo $G$ denotamos $\bar{R}(G)$ a relação de equivalência em $G_{0}$ gerada por $R(G)$, então:

(i) Dado $F: G \rightarrow G^{\prime}$ homomorfismo $\left(F_{0} \times F_{0}\right)_{*}(\bar{R}(G)) \subseteq \bar{R}\left(G^{\prime}\right)$.

(ii) Existe um funtor, que se restringe aos universos, $\Gamma: G R A \rightarrow S E T$ tal que $\Gamma(G \stackrel{F}{\rightarrow}$ $\left.G^{\prime}\right)=G_{0} / \bar{R}(G) \stackrel{\bar{F}_{0}}{\rightarrow} G_{0}^{\prime} / \bar{R}\left(G^{\prime}\right)$.

Definimos o funtor "componentes conexas" $\pi_{0}: C A T \rightarrow S E T$ como a composição do funtor "esquecimento" $\mathcal{E}: C A T \rightarrow G R A$ com o funtor $\Gamma^{\prime}$, naturalmente isomorfo a $\Gamma$ : $G R A \rightarrow S E T$, tal que $\forall G \in \operatorname{obj}(G R A) \Gamma^{\prime}(G)=\{H \in \operatorname{obj}(G R A): H$ é subgrafo pleno, não vazio, de $G$ determinado por alguma classe de objetos $\left.H_{0} \in G_{0} / \bar{R}(G)\right\}$. Temos que:

(i) $\pi_{0}$ se restringe aos universos : $\pi_{0}: U-C A T \rightarrow U-S E T$. 
(ii) $\pi_{0}(\emptyset)=\emptyset$.

(iii) $\forall C \in \operatorname{obj}(C \cdot A T)\left(\left(\pi_{0}\left(C^{*}\right) \cong \pi_{0}(C)\right)\right)$.

(iv) Sejam $\mathcal{D}: S E T \rightarrow C A T$ o funtor "categoria discreta" e 2 o grafo $0 \stackrel{(0,1)}{\rightarrow} 1$; então can $: C \rightarrow \mathcal{D}\left(\pi_{0}(C)\right)$ determina em $C A T$ um coigualador de $\epsilon v_{0}, \epsilon v_{1}: C^{2} \rightarrow C$ e $\pi_{0}$ é adjunto à esquerda de $\mathcal{D}$.

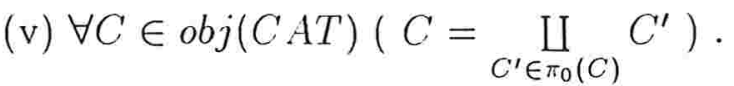

(vi) Dado $F: I \rightarrow C$ funtor, $\pi_{0}((c \rightarrow F)) \cong \underset{I}{\lim } C(c, F(-))$ natural em $c \in\left(C^{\star}\right)_{0}$ e, como $(F \rightarrow c)^{\star} \cong\left(c \rightarrow F^{\star}\right)$, temos $\pi_{0}((F \rightarrow c)) \underset{I^{\star}}{\lim } C(F(-), c)$ natural em $c \in C_{0}$.

Sejam $T: I \rightarrow I^{\prime}$ funtor. $T$ é funtor final $\Longleftrightarrow \forall i^{\prime} \in \operatorname{obj}\left(I^{\prime}\right)\left(\left(i^{\prime} \rightarrow T\right)\right.$ é não vazia e conexa $) \stackrel{I, I^{\prime} \in(C A T)_{0}}{\Longleftrightarrow} \pi_{0}(((-) \rightarrow T)) \cong \Delta_{I^{\prime *}}(1): I^{\prime \star} \rightarrow S E T$. Temos então:

(i) $\Delta_{1}(c): 1 \rightarrow C$ é funtor final $\Longleftrightarrow c \in C_{0}$ é objeto final.

(ii) can : $C \rightarrow \mathcal{D}\left(\pi_{0}(C)\right)$ é funtor final.

(iii) $1_{I^{\prime}}: I^{\prime} \rightarrow I^{\prime}$ é final.

(iv) $T: I \rightarrow I^{\prime} ; T^{\prime}: I^{\prime} \rightarrow I^{\prime \prime}$ finais $\Rightarrow T^{\prime} \circ T: I \rightarrow I^{\prime \prime}$ final.

(v) Sejam $T: I \rightarrow I^{\prime}$ e $T^{\prime}: I^{\prime} \rightarrow I^{\prime \prime}$ funtores; então:

. $T$ final $\wedge T^{\prime} \circ T$ final $\Rightarrow T^{\prime}$ final.

. $T^{\prime}$ hom-bijetor $\wedge T^{\prime} \circ T$ final $\Rightarrow T$ final.

. $T^{\prime}$ hom-bijetor $\Rightarrow\left(T^{\prime} \circ T\right.$ final $\Longleftrightarrow\left(T\right.$ final $\wedge T^{\prime}$ final $\left.)\right)$.

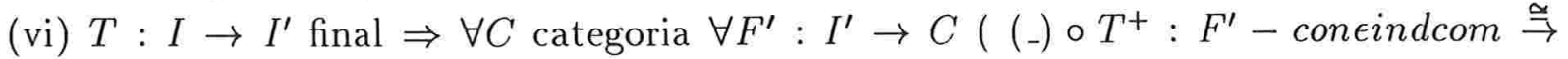

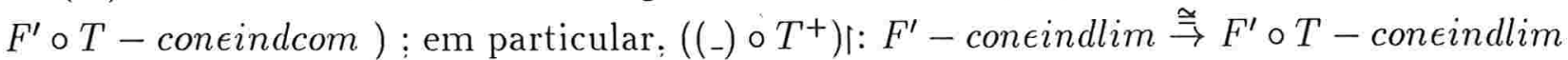
, $\log$ o $F^{\prime}$ admite limite indutivo $\Longleftrightarrow F^{\prime} \circ T$ admite limite indutivo e, quando existem, são canonicamente isomorfos can $\underset{I}{\lim } F^{\prime} \circ T \underset{I^{\prime}}{\cong} \lim F^{\prime}$.

Sejam $\mathcal{I}, \mathcal{I}^{\prime} \subseteq \operatorname{obj}(C A T): \mathcal{I} \preceq \mathcal{I}^{\prime} \Longleftrightarrow \forall I^{\prime} \in \mathcal{I}^{\prime} \exists I \in \mathcal{I} \exists T: I \rightarrow I^{\prime}$ (T é final). Assim:

(i) Dado $U$ universo, $\preceq$ é pré-ordem em $\mathcal{P}(\operatorname{obj}(U-C A T))$.

(ii) Dado $M: C \rightarrow C^{\prime}$ funtor, se $M$ preserva limites indutivos de tipo $\mathcal{I}$ então $M$ preserva limites indutivos de tipo $\mathcal{I}^{\prime}$.

Dualmente definimos: $T: I \rightarrow I^{\prime}$ é funtor inicial $\Longleftrightarrow \forall i^{\prime} \in \operatorname{obj}\left(I^{\prime}\right)\left(\left(T \rightarrow i^{\prime}\right)\right.$ é não vazia e conexa ). Analogamente temos $T: I \rightarrow I^{\prime}$ inicial $\Rightarrow \forall C$ categoria $\forall F^{\prime}: I^{\prime} \rightarrow C$ ( - ० $T^{-}: F^{\prime}-$ coneprojcom $\stackrel{\cong}{\rightrightarrows} F^{\prime} \circ T-$ coneprojcom ).

Notemos que:

(i) $I \neq \emptyset \wedge T: I \rightarrow I^{\prime}$ localmente bijetor $\Rightarrow \forall i \in I_{0}((T(i) \rightarrow T)$ possui objeto inicial $\wedge$ $(T \rightarrow T(i))$ possui objeto final $)$.

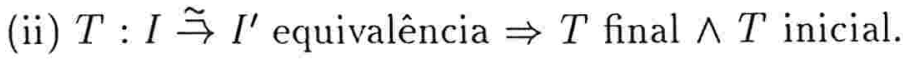




\subsection{Mais categorias!}

Colocamos mais alguns desenvolvimentos da teoria das categorias necessários ao trabalho. As referências básicas são [159] : [186] . Constam ainda referências adicionais em cada ítem.

\subsubsection{Adjunção}

Neste ítem tratamos de algumas noções correlatas a de adjunção .

Abaixo definimos adjunção relativa a um funtor dado.

Referência adicional : [198] .

Definição 1 : Sejam $A^{\prime}, A, B$ categorias, $A, B$ localmente pequenas e os funtores $J$ : $A^{\prime} \rightarrow A, G: B \rightarrow A, F: A^{\prime} \rightarrow B$.

(i) $F$ é $J$-adjunto à esquerda de $G \Longleftrightarrow B\left(F_{--}\right) \cong A\left(J_{-}, G_{-}\right): A^{\prime *} \times B \rightarrow S E T$.

(ii) $F$ é $J$-adjunto à direita de $G \Longleftrightarrow B\left({ }_{-}, F_{-}\right) \cong A\left(G_{-}, J_{-}\right): B^{\star} \times A^{\prime} \rightarrow S E T$.

Observação 1 :

(i) Diferentemente do caso clássico $\left(J=1_{A}: A \rightarrow A\right)$ tem-se :

- Perda da simetria: $F J$-adjunto à esquerda (direita) de $G \nRightarrow G J$-adjunto à direita (esquerda) de $F$.

- Em geral, $F$ não determina $G$ : existem funtores $J, F, G, H$ tais que $F$ é $J$-adjunto à esquerda (direita) de $G$; $H: B \rightarrow A$ mas $G \not H$.

(ii) Preservam-se algumas propriedades:

. $F$ é $J$-adjunto à esquerda (direita) de $G \Longleftrightarrow \exists \eta \in\left[A^{\prime}, A\right](J ; G \circ F)\left(\exists \epsilon \in\left[A^{\prime}, A\right](G \circ\right.$ $F, J)$ ) "universal"; $\operatorname{logo} G$ determina $F$ a menos de isomorfismo.

. $G$ possui $J$-adjunto à esquerda (direita) $\Longleftrightarrow \forall a^{\prime} \in\left(A^{\prime}\right)_{0} \exists\left(F_{0}\left(a^{\prime}\right) ; \eta_{a^{\prime}}\right) \in \operatorname{obj}\left(J\left(a^{\prime}\right) \rightarrow\right.$ $G)$ objeto inicial $\left(\exists\left(F_{0}\left(a^{\prime}\right), \epsilon_{a^{\prime}}\right) \in \operatorname{obj}\left(G \rightarrow J\left(a^{\prime}\right)\right)\right.$ objeto final).

- Dados $F J$-adjunto à esquerda de $G$ e $i: X^{+} \rightarrow A^{\prime}$ cone indutivo limite então $J \circ i$ limite $\Rightarrow F \circ i$ limite.

- Dados $F J$-adjunto à direita de $G$ e $p: X^{-} \rightarrow A^{\prime}$ cone projetivo limite então $J \circ p$ limite $\Rightarrow F \circ p$ limite.

(iii) Seja o diagrama de categorias e funtores: 


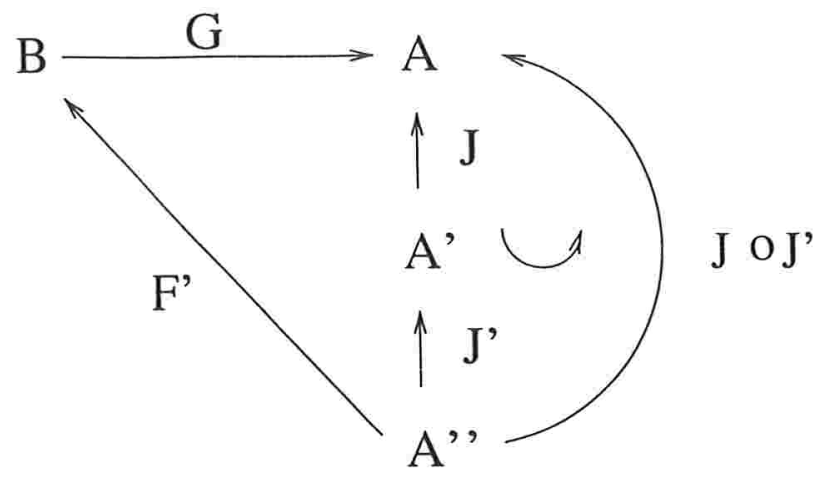

Assumimos que:

. $B, A$ são localmente pequenas.

- Para todo $a^{\prime} \in\left(A^{\prime}\right)_{0}$ existe (e pelo axioma da escolha forte fixemos algum !) $\Gamma_{a^{\prime}}$ : $X_{a^{\prime}} \rightarrow A^{\prime \prime}$ tal que $a^{\prime} \cong \underset{X_{a^{\prime}}}{\lim } J^{\prime} \circ \Gamma_{a^{\prime}}$.

. $J$ preserva os limites dados acima.

. $F^{\prime}$ é $J \circ J^{\prime}$-adjunto à esquerda de $G$.

Então são equivalentes:

. $\exists F J$-adjunto à esquerda de $G$ (neste caso $F \circ J^{\prime} \cong F^{\prime}$ ).

. $\forall a^{\prime} \in\left(A^{\prime}\right)_{0} \exists \underset{X_{a^{\prime}}}{\lim } F^{\prime} \circ \Gamma_{a^{\prime}}$.

(iv) Dado $G: \stackrel{B}{B} \rightarrow A$ sejam $[G]_{l}=\left\{a \in A_{0}:(a \rightarrow G)\right.$ possui objeto inicial $\} ; A^{\prime}$ a subcategoria plena de $A$ com $\left(A^{\prime}\right)_{0}=[G]_{l}, J: A^{\prime} \hookrightarrow A$ e $F: A^{\prime} \rightarrow B$ adjunto parcial máximo de $G$ ( $J$-adjunto a esquerda de $G$ ) então:

. $A^{\prime}$ é fechada por isomorfismos.

. $\forall \Gamma: X \rightarrow A^{\prime}(\underset{X}{\exists} \underset{\underset{X}{\lim }}{\log } F \circ \Gamma \Rightarrow(J$ cria limites indutivos para $\Gamma \wedge F$ preserva $\Gamma$-limites indutivos)).

Proposição $1:$ Sejam $C: A, X \subseteq(C A T)_{0}, J: C \rightarrow A, \Gamma: X \rightarrow C A T: W=\underset{X}{\lim } \Gamma$, $\forall x \in X_{0}\left(j_{x}: \Gamma(x) \rightarrow W\right), F: W \rightarrow C$.

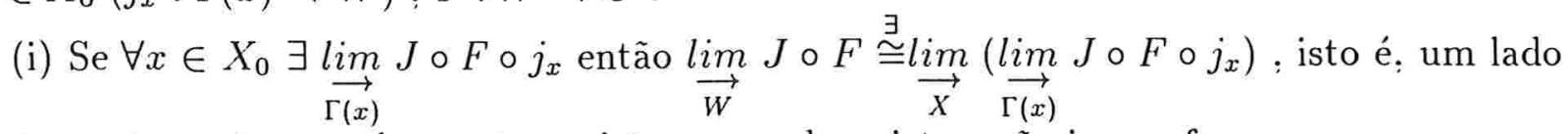
existe exatamente quando o outro existe e quando existem são isomorfos.

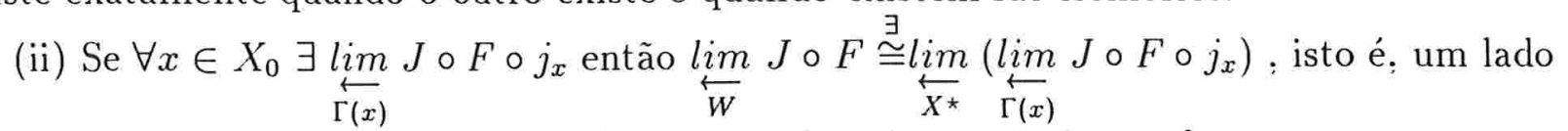
existe exatamente quando o outro existe e quando existem são isomorfos.

(i) Sejam $J^{\rightarrow}: A \rightarrow(C A T \rightarrow C)$ como no ítem (1.2.3) e $L$ seu adjunto à esquerda, parcial, máximo, então $\forall H: Z \rightarrow C \in(C A T \rightarrow C)_{0}\left([Z, A]\left(J \circ H: \Delta_{Z}(-)\right) \cong(C A T \rightarrow C)\left(H, J^{\rightarrow}\left({ }_{-}\right)\right)\right.$ 
). portanto $H \in\left[J^{\rightarrow}\right]_{l} \Longleftrightarrow \exists \underset{Z}{\exists} \lim _{\vec{Z}} J \circ H$ e $L(H) \underset{Z}{\stackrel{\exists}{\cong} \lim } J \circ H$. Como $C A T$ é $V$-ind completa e $P_{C}:(C A T \rightarrow C) \rightarrow C . A T$ cria todos limites indutivos $F \cong \underset{x}{\lim }\left(F \circ j_{x}\right)$. Utilizando a

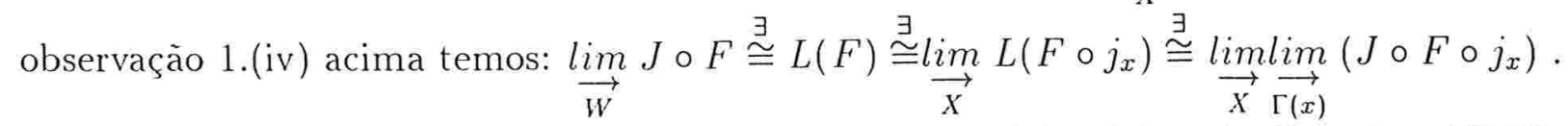

(ii) Dualmente a (i), utilizando o adjunto à direita parcial máximo de $J^{\leftarrow}: A \rightarrow(C A T \rightarrow$ $C)^{\star}$.

Consideramos abaixo outra aplicação importante das adjunções relativas : as extensões de Kan pontuais. As hipóteses de tamanho necessárias serão geralmente omitidas ${ }^{3} S E T, C A T$ podem designar $V-S E T$. $V-C A T$ ou $U-S E T, U-C A T$ para algum universo $U$.

Referências adicionais : [127] : [092] .

Definição 2 : Dado o funtor $J: C \rightarrow A$ consideramos, para cada categoria $B$, o funtor:

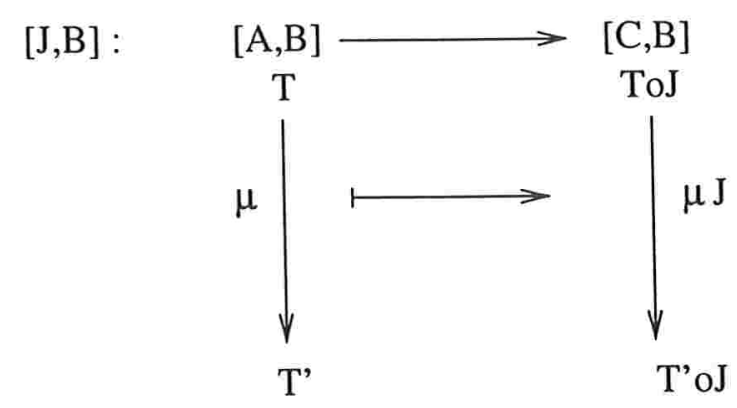

Dizemos que $F: C \rightarrow B$ admite extensão de Kan à esquerda (direita) através de $J$ quando $F \in[[J, B]]_{l}\left(F \in[[J, B]]_{r}\right)$; neste caso denotamos $L_{J}(F): A \rightarrow B\left(R_{J}(F): A \rightarrow B\right)$ o; único a menos de isomorfismo, valor do adjunto à esquerda (direita) parcial de $[J ; B]$ em $F$ e $\eta_{F}: F \rightarrow L_{J}(F) \circ J$ sua unidade em $F\left(\epsilon_{F}: R_{J}(F) \circ J \rightarrow F\right.$ sua co-unidade em $\left.F\right)$.

Observação 2 :

(i) $\forall c \in C_{0}\left(L_{J}(C(c,-)) \cong A(J(c),-)\right)$.

(ii)

$$
\begin{array}{rl}
\mathrm{L}_{\mathrm{J}}\left(\mathrm{Y}_{\mathrm{C}}\right) \cong \tilde{\mathrm{J}}: \mathrm{A} \longrightarrow & {\left[\mathrm{C}^{*}, \mathrm{SET}\right]} \\
\mathrm{A}\left(\mathrm{J}\left(\_\right), \mathrm{a}\right) & \\
\mathrm{f} \mid & \downarrow \mathrm{A}\left(\mathrm{J}\left(\_\right), \mathrm{f}\right) \\
\mathrm{b} & \mathrm{A}\left(\mathrm{J}\left(\_\right), \mathrm{b}\right)
\end{array}
$$

${ }^{3}$ Assumir que as categorias são localmente pequenas é conveniente. 
(iii) Dado $J: C \stackrel{!}{\rightarrow} 1$ então $\exists L_{J}(F) \Longleftrightarrow \exists \stackrel{\lim }{\longrightarrow} F$.

(iv) Dados $J: C \rightarrow A, F: C \rightarrow B$ temos bijeções : $\left[C^{\star}: S E T\right](\tilde{J}(a), \tilde{F}(b)] \cong[J \rightarrow$ $a, B]\left((J \rightarrow a) \stackrel{P(a)}{\rightarrow} C \stackrel{F}{\rightarrow} B, \Delta_{(J \rightarrow a)}(b)\right)$ naturais em $a \in A_{0}^{\star}$ e $b \in B_{0}$.

(v) Dados $J: C \rightarrow A, F: C \rightarrow B: T: A \rightarrow B$ temos bijeção: $[C: B](F, T \circ J) \cong$ $\left[A^{*} \times B, S E T\right]\left(B\left(T\left(_{-}\right)_{-}\right)\right.$: $\left.\left[C^{\star}: S E T\right]\left(\tilde{J}\left({ }_{-}\right), \tilde{F}\left({ }_{-}\right)\right)\right)$. Se $\eta: F \rightarrow T \circ J$ corresponde a um isomorfismo então $T$ é extensão de Kan à esquerda de $F$ através de $J$ (com unidade $\eta: F \rightarrow[J, B](T))$

Proposição $2:$ Dados $J: C \rightarrow A, F: C \rightarrow B, T: A \rightarrow B$ são equivalentes:

(i) $T$ é $\tilde{J}$-adjunto à esquerda de $\tilde{F}$.

(ii) $\left[C^{\star}, S E T\right](\tilde{J}(a), \tilde{F}(-)] \cong B(T(a)$; $)$ natural em $a \in A_{0}^{\star}$.

(iii) $\forall a \in A_{0}\left(\exists \underset{\lim }{\rightarrow}\left((J \rightarrow a) \stackrel{P_{a}}{\rightarrow} C \stackrel{F}{\rightarrow} B\right) \cong T(a)\right)$.

Quando as condições acima estão satisfeitas dizemos que $T$ é extensão de Kan à esquerda; pontual, de $F$ através de $J$.

Observação 3 :

(i) Se $C$ é pequena e $A$ localmente pequena então para toda categoria $B(C A T)_{0}$-ind completa $[J, B]$ tem adjunto à esquerda.

(ii) Se $J$ é hom-bijetor e $L_{J}(F)$ existe e é pontual então a flecha universal $\eta_{F}: F \stackrel{\cong}{\rightarrow}$ $L_{J}(F) \circ J$ é um isomorfismo natural.

(iii) Se $L_{J}(F)$ existe e é pontual então $L_{J}(F): A \rightarrow B$ preserva todos os limites indutivos preservados por $\tilde{J}: A \rightarrow\left[C^{\star}, S E T\right] \operatorname{logo}$; como $\left[C^{\star}, S E T\right]$ tem limites indutivos pequenos e esses são pontuais, $L_{J}(F)$ preserva todos os limites indutivos pequenos que são preservados por $A(J(c):-): A \rightarrow S E T$ para todos $c \in C_{0}^{\star}$.

Proposição 3 : Dado $J: C \rightarrow A$ são equivalentes:

(i) $\forall a \in A_{0}(\exists \underset{(J \rightarrow a)}{\lim }(J \circ P(a)) \cong a)$.

(ii) $1_{A}$ é extensão de Kan à esquerda, pontual, de $J$ através de $J$ com unidade $1_{J}: J \rightarrow$ $1_{A} \circ J$.

(iii) $\tilde{J}: A \rightarrow\left[C^{\star}, S E T\right]$ é hom-bijetor.

Quando as condições acima estão satisfeitas dizemos que $J: C \rightarrow A$ é denso.

Observação 4 :

(i) $Y_{C}: C \rightarrow\left[C^{\star}, S E T\right]$ é denso.

(ii) Dado $J: C \rightarrow A\left(J\right.$ hom-bijetor $\left.\Rightarrow \tilde{J} \circ J \cong Y_{C}: C \rightarrow\left[C^{\star}, S E T\right]\right)$.

(iii) Sejam $H: C \rightarrow B, F: B \rightarrow A$ com $F$ hom-bijetor então $F \circ H$ denso $\Rightarrow F$; $H$ densos.

Proposição 4 : Dada $(L, R, \eta, \epsilon): A \rightarrow B$ adjunção são equivalentes:

(i) $L: A \rightarrow B$ é denso. 
(ii) $R: B \rightarrow A$ é hom-bijetor.

(iii) $\epsilon: L \circ R \cong 1_{B}$.

Quando as condições acima estão satisfeitas dizemos que $(L, R): A \rightarrow B$ é uma reflexão (de $A$ em $B$ ).

Proposição 5 : Sejam $(L, R): A \rightarrow B$ reflexão e $\Gamma: X \rightarrow B$; então:

(i) $\exists \lim (R \circ \Gamma) \Rightarrow \exists \underset{\lim }{\longrightarrow}(\Gamma)$.

(ii) $\exists \overleftrightarrow{\lim }(R \circ \Gamma) \Rightarrow \exists \overrightarrow{\lim }(\Gamma)$.

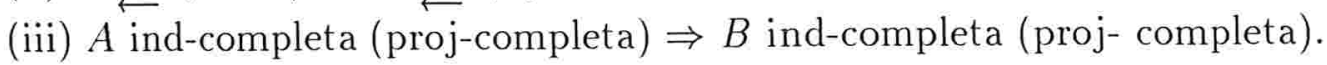

A seguir expomos generalizações da noção de adjunção em outra direção .

Referências adicionais : [063] : [174] .

Proposição 6 : Sejam $C$ categoria localmente pequena, $I$ categoria pequena e $F: I \rightarrow C$ funtor.

(i) São equivalentes (e caso ocorram dizemos que $F$ é um diagrama pequeno fracamente inicial de $C$ ) :

. $\forall c \in C_{0} \exists i \in I_{0} \exists f_{i} \in C(F(i), c)$.

- $\forall c \in C_{0}\left(\pi_{0}(F \rightarrow c) \neq \emptyset\right)$.

. $\exists \eta: \Delta_{C}(1) \rightarrow \underset{I^{\star}}{\lim } C(F(i):-)$.

. $\exists \eta: \Delta_{C}(1) \rightarrow \underset{\mathcal{D}\left(I_{0}\right)}{\amalg} C(F(i) ;-)$.

. $\forall c \in C_{0}((F \rightarrow c) \neq \emptyset)$.

(ii) São equivalentes (e caso ocorram dizemos que $F$ é um diagrama pequeno localmente inicial de $C$ ) :

. $\forall c \in C_{0} \exists i \in I_{0} \exists f_{i} \in C(F(i), c) \wedge\left(\forall\left(i, f_{i}\right) \forall\left(j, g_{j}\right)\left(\left(i, j \subseteq I_{0} \wedge f_{i} \in C(F(i), c) \wedge\right.\right.\right.$ $\left.g_{j} \in C(F(j), c)\right) \Rightarrow \exists n \in \omega-0 \exists H: Z Z_{n} \rightarrow \mathcal{E}(I)$ (onde $Z Z_{n}$ é o grafo $0 \rightarrow 1 \leftarrow 2 \rightarrow \ldots \leftarrow$ $2 n-2 \rightarrow 2 n-1 \leftarrow 2 n) \exists \gamma$ cone indutivo comutativo $(\operatorname{base}(\gamma)=\mathcal{E}(F) \circ H \wedge$ vértice $(\gamma)=c \wedge$ $\left.\left.H(0)=i \wedge H(2 n)=j \wedge \gamma\left(\iota_{0}\right)=f_{i} \wedge \gamma\left(\iota_{2 n}\right)=g_{j}\right)\right)$.

. $\forall c \in C_{0}\left(\pi_{0}(F \rightarrow c) \cong 1\right)$.

. $\exists \eta: \Delta_{C}(1) \stackrel{\cong}{\underset{I^{*}}{\rightarrow}} \lim \left(F(i)_{;--}\right)$.

(iii) Se $I$ é discreta são equivalentes (e caso ocorram dizemos que $F$ é o (essencialmente único) diagrama pequeno multiplamente inicial de $C$ ) :

. $\forall c \in C_{0} \exists ! i \in I_{0} \exists ! f_{i} \in C(F(i), c)$.

. $\forall c \in C_{0}\left(\pi_{0}(F \rightarrow c) \cong 1\right)$.

. $\exists \eta: \Delta_{C}(1) \stackrel{\cong}{\rightrightarrows} \coprod_{I^{\star}} C(F(i),-)$.

. $\exists \Psi: I_{0} \cong \pi_{0}(C) \wedge \forall i \in I_{0}(F(i)$ é objeto inicial de $\Psi(i))$. 
. $\forall c \in C_{0}((F \rightarrow c) \cong 1)$.

(iv) se $I \cong 1$ são equivalentes (e caso ocorram dizemos que $F$ é o (essencialmente único) diagrama (pequeno) inicial de $C$ ) :

. $\forall c \in C_{0} \exists ! f \in C(F(i), c)$.

. $\forall c \in C_{0}\left(\pi_{0}(F \rightarrow c) \cong 1\right)$.

. $\exists \eta: \Delta_{C}(1) \rightarrow \underset{I^{\star}}{\lim } C(F(i),-)$.

- $F(i)$ é objeto inicial de $C$.

- $\forall c \in C_{0}((F \rightarrow c) \cong \mathbf{1})$.

Exemplo : Seja $K c$ a categoria dos corpos comutativos então $C A R: \mathcal{D}(\{p \in \mathbb{N}: p=0 \vee p$ é primo $\}) \rightarrow K c: C A R(0)=\mathbb{Q}, p$ primo $\Rightarrow C A R(p)=\mathbb{Z} / p \mathbb{Z}$ é "o" diagrama (pequeno) multiplamente inicial de $K c$.

Proposição 7 : Seja $A$ categoria localmente pequena.

(i) $\left(\forall P \in(C A T)_{0}\left(P \cong \mathcal{D}\left(P_{0}\right)\right) \Rightarrow A\right.$ é $P$-proj completa $) \Rightarrow$ ( $A$ possui objeto inicial $\Longleftrightarrow A$ possui diagrama pequeno multiplamente inicial $)$.

(ii) $\left(\forall P \in(C A T)_{0}\left(\pi_{0}(P) \cong 1 \Rightarrow A\right.\right.$ é $P$ - proj completa $\left.)\right) \Rightarrow(A$ possui diagrama pequeno multiplamente inicial $\Longleftrightarrow A$ possui diagrama pequeno fracamente inicial) (notar que $\forall a \in A_{0}$ a subcategoria plena de $A$ dos objetos "abaixo de $a$ " tem objeto inicial !).

Definição 3 : Sejam $C$ categoria localmente pequena e $T: C \rightarrow S E T$ funtor.

(i) Um diagrama pequeno fracamente representante de $T$ é um diagrama pequeno fracamente inicial de $(1 \rightarrow T)$, ou equivalentemente, um par $(L, \eta)$ tal que $L: X \rightarrow C$ é funtor, $X$ é categoria pequena e $\eta \underset{X^{\star}}{\lim } C(L(x),-) \rightarrow T$.

(ii) Um diagrama pequeno localmente representante de $T$ é um diagrama pequeno localmente inicial de $(1 \rightarrow T)$; ou equivalentemente, um par $(L, \eta)$ tal que $L: X \rightarrow C$ é funtor; $X$ é categoria pequena e $\eta \underset{X^{\star}}{\lim } C\left(L(x)_{:-}\right) \stackrel{\cong}{\rightarrow} T$. Dizemos multiplamente representante (representante) quando $X \cong \mathcal{D}\left(X_{0}\right)(X \cong 1)$.

Proposição 8 : Sejam $C$ categoria localmente pequena e $T: C \rightarrow S E T$ funtor.

(i) $C$ possui limites projetivos pequenos $\Rightarrow$ ( $T$ representável $\Longleftrightarrow$ ( $T$ admite diagrama pequeno fracamente representante e $T$ preserva limites projetivos pequenos )).

(ii) $C$ possui limites projetivos pequenos conexos e não vazios $\Rightarrow$ ( $T$ admite diagrama pequeno multiplamente representante $\Longleftrightarrow$ ( $T$ admite diagrama pequeno fracamente representante e $T$ preserva limites projetivos pequenos conexos e não vazios ) ) ( que $T$ necessariamente preserva limites projetivos pequenos conexos e não vazios segue da proposição 8 do ítem (1.3.2) ). 
Definição 4 : Sejam $A, B$ categorias localmente pequenas e $R: B \rightarrow A$ funtor.

(i) Um diagrama pequeno fracamente livre para $R$ em $a \in A_{0}$ é um diagrama pequeno fracamente representante de $A(a, R(-)): B \rightarrow S E T$, ou seja, um par $(L, \eta)$ tal que $L: X \rightarrow$ $B$ é funtor, $X$ é categoria pequena e $\eta \underset{X^{*}}{\lim } B(L(x),-) \rightarrow A(a, R(-))$.

(ii) Um diagrama pequeno localmente livre para $R$ em $a \in A_{0}$ é um diagrama pequeno localmente representante de $A(a, R(-)): B \rightarrow S E T$, ou seja, um $\operatorname{par}(L, \eta)$ tal que $L: X \rightarrow B$ é funtor, $X$ é categoria pequena e $\eta \underset{X^{\star}}{\lim } B(L(x),-) \stackrel{\cong}{\rightarrow} A(a, R(-))$. Dizemos multiplamente livre (livre) quando $X \cong \mathcal{D}\left(X_{0}\right)(X \cong 1)$.

Exemplo: Seja $J: K c \hookrightarrow A u c$ o funtor de inclusão da categori dos corpos comutativos na categoria dos anéis com unidade e comutativos. $J$ tem "multi-adjunto à esquerda pequeno" (diagrama pequeno multiplamente livre para todo $\left.a \in(A u c)_{0}\right)$ dado, em $a \in(A u c)_{0}$; por:

$$
F_{a}: \mathcal{D}(\operatorname{Spec}(a)) \rightarrow(a \rightarrow J): p \mapsto(a \stackrel{\operatorname{proj}(p)}{\rightarrow} a / p \stackrel{i n j(p)}{\rightarrow} \operatorname{Frac}(a / p))
$$

Proposição 9 : Sejam $A, B$ categorias localmente pequenas e $R: B \rightarrow A$ funtor.

(i) $B$ possui limites projetivos pequenos $\Rightarrow\left(\forall a \in A_{0}\right.$ ( $R$ admite adjunto à esquerda em $a) \Longleftrightarrow\left(\forall a \in A_{0}(R\right.$ admite diagrama pequeno fracamente livre em $a$ e $R$ preserva limites projetivos pequenos ) ).

(ii) $B$ possui limites projetivos pequenos conexos e não vazios $\Rightarrow$ ( $\forall a \in A_{0}$ ( $R$ admite diagrama pequeno multiplamente livre em $a) \Longleftrightarrow\left(\forall a \in A_{0}\right.$ ( $R$ admite diagrama pequeno fracamente livre em $a$ e $R$ preserva limites projetivos pequenos conexos e não vazios ) ) .

Definição 5 : Sejam $I$ categoria pequena, $C$ categoria localmente pequena e $F: I \rightarrow C$ funtor.

(i) Um diagrama pequeno fracamente limite indutivo de $F$ é um diagrama pequeno fracamente livre para $\Delta_{I}: C \rightarrow C^{I}$ em $F$; ou seja, um par $(\Gamma, \eta)$ tal que $\Gamma: X \rightarrow C$ é funtor, $X$ é categoria pequena e $\eta \underset{X^{\star}}{\lim } C(\Gamma(x),-) \rightarrow[I, C]\left(F: \Delta_{I}(-)\right)$.

(ii) Um diagrama pequeno localmente limite indutivo de $F$ é um diagrama pequeno localmente livre para $\Delta_{I}: C \rightarrow C^{I}$ em $F$ : ou seja, um par $(\Gamma, \eta)$ tal que $\Gamma: X \rightarrow C$ é funtor: $X$ é categoria pequena e $\eta \underset{X^{\star}}{\lim } C(\Gamma(x),-) \stackrel{\cong}{\rightrightarrows}[I, C]\left(F, \Delta_{I}(-)\right)$. Dizemos multiplamente limite indutivo (limite indutivo) quando $X \cong \mathcal{D}\left(X_{0}\right)(X \cong 1)$.

Exemplo : $K c$ tem "multi-coproduto pequeno" de pares de objetos $F, F^{\prime}$ dado pelo conjunto indexado por $p \in \operatorname{Sp\epsilon c}\left(F \otimes_{\mathbb{Z}} F^{\prime}\right)$ : 


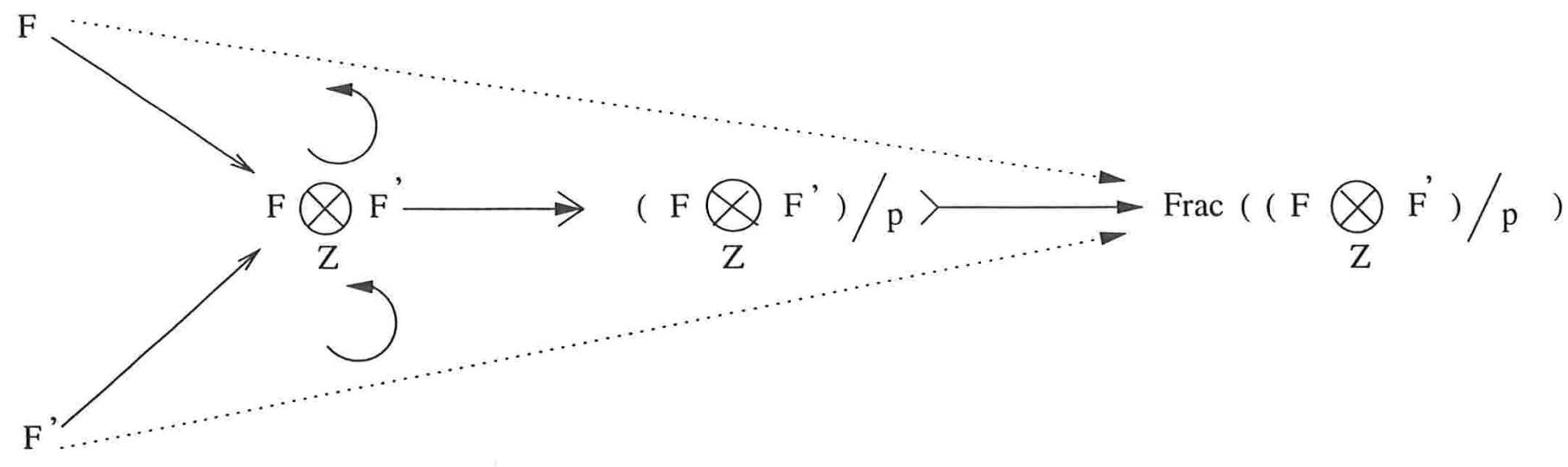

\subsubsection{Comutação de limites}

Referências adicionais: [084], [092] , [208] .

Observação 1 : Seja $C$ categoria. Como $\forall X \in \operatorname{obj}(C A T)(\operatorname{con} \in \operatorname{proj} \operatorname{com}(X, C)=$ $C^{\left(X^{-}\right)} \wedge \operatorname{con} \epsilon i n d \operatorname{com}(X, C)=C^{\left(X^{+}\right)}$e se $C$ é $X$-projetivamente completa $(X$-indutivamente completa) então as categorias exponenciais de "base $C$ " são pontualmente $X$-proj completas ( $X$-ind completas) temos que:

(i)Se $C$ é $X, Y$-projetivamente completa então são equivalentes:

. $\forall F: X \times Y \rightarrow C\left(\operatorname{can}(F): \underset{X}{\lim } \underset{Y}{\stackrel{l i m}{i m}} F(x, y) \stackrel{\cong}{\rightrightarrows} \lim _{Y} \underset{X}{\stackrel{l i m}{m}} F(x, y)\right)$

- $\operatorname{con} \epsilon \operatorname{proj} \operatorname{com}(X, C)$ é $Y$-proj completa e conєprojlim(X,C) $\hookrightarrow \operatorname{con} \epsilon \operatorname{proj} \operatorname{com}(X, C)$ é fechada por limites projetivos de tipo $Y$.

- $\operatorname{con} \epsilon \operatorname{proj} \operatorname{com}(Y, C)$ é $X$-proj completa e contprojlim $(Y, C) \hookrightarrow \operatorname{con} \epsilon \operatorname{proj} \operatorname{com}(Y, C)$ é fechada por limites projetivos de tipo $X$.

Estes sempre ocorrem ("limites projetivos comutam com limites projetivos").

(ii)Se $C$ é $X, Y$-indutivamente completa então são equivalentes:

. $\forall F: X \times Y \rightarrow C(\operatorname{can}(F) \underset{X}{: \underset{Y}{\lim } \underset{Y}{\lim }} F(x, y) \stackrel{\cong}{\rightarrow} \underset{Y}{\lim } \underset{X}{\longrightarrow} \lim F(x, y))$.

- $\operatorname{con} \epsilon i n d \operatorname{com}(X, C)$ é $Y$-ind completa e contindlim $(X, C) \hookrightarrow \operatorname{con} \in \operatorname{ind} \operatorname{com}(X, C)$ é fechada por limites indutivos de tipo $Y$.

. $\operatorname{con} \epsilon i n d \operatorname{com}(Y, C)$ é $X$-ind completa e con $\epsilon i n d l i m(Y, C) \hookrightarrow \operatorname{con} \epsilon i n d \operatorname{com}(Y, C)$ é fechada por limites indutivos de tipo $X$.

Estes sempre ocorrem ("limites indutivos comutam com limites indutivos").

(iii)Se $C$ é $P$-proj completa e $I$-ind completa então são equivalentes:

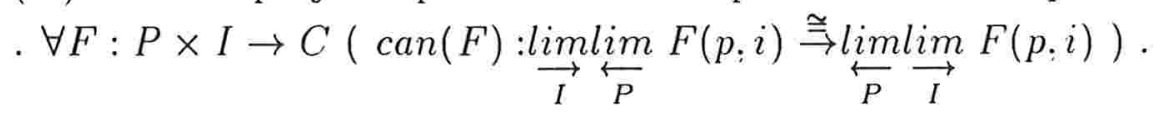


- $\operatorname{con} \epsilon \operatorname{proj} \operatorname{com}(P, C)$ é $I$-ind $\operatorname{completa}$ e $\operatorname{con} \epsilon \operatorname{projlim}(P, C) \hookrightarrow \operatorname{con} \epsilon \operatorname{proj} \operatorname{com}(P: C)$ é fechada por limites indutivos de tipo $I$.

. coneindcom( $I, C)$ é $P$-proj completa e contindlim $(I, C) \hookrightarrow \operatorname{con} \epsilon i n d c o m(I, C)$ é fechada por limites projetivos de tipo $P$.

Quando estes ocorrem dizemos que, em $C$, $I$-limites indutivos comutam com $P$-limites projetivos.

Observação 2 : Situações semelhantes ocorrem quando consideramos limites em $C$ indexados por grafos $G \in \operatorname{obj}(G R A)$ pois as categorias de cones comutativos e cones são (isomorfas a) categorias exponenciais: $\operatorname{con} \epsilon \operatorname{proj} \operatorname{com}(G, C) \cong C^{\left(\mathcal{L}(G)^{-}\right)} \cdot \operatorname{con} \epsilon \operatorname{ind} \operatorname{com}(G, C) \cong$ $C^{\left(\mathcal{L}(G)^{+}\right)}, \operatorname{con} \in \operatorname{proj}(G, C)=C^{\left(G^{-}\right)} \cong C^{\mathcal{L}\left(G^{-}\right)}, \operatorname{con} \in i n d(G, C)=C^{\left(G^{+}\right)} \cong C^{\mathcal{L}\left(G^{+}\right)}$.

Definição 1 : Dados $\mathcal{P}, \mathcal{I} \subseteq \operatorname{obj}(C A T)$ e $C$ categoria $\mathcal{P}$-proj completa $\mathcal{I}$-ind completa denotamos $I S O(C, \mathcal{P})=\{X \in \operatorname{obj}(C A T): C$ é $X$-ind completa e $\forall P \in \mathcal{P} \forall F: P \times X \rightarrow C($

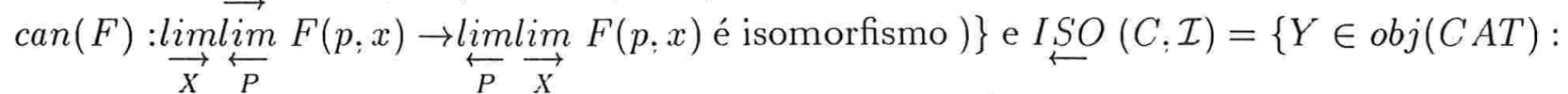

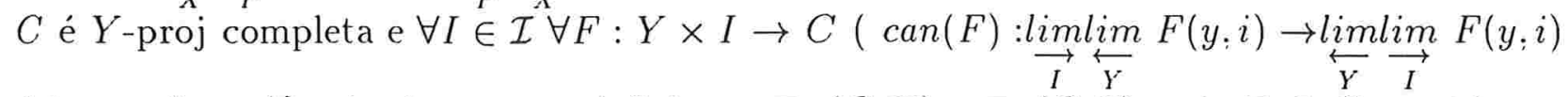
é isomorfismo $)\}$. Analogamente definimos $\underset{\subseteq}{\leftrightarrows}(C, \mathcal{P}): \underset{\longleftarrow}{\Sigma}(C, \mathcal{I})$ onde $\Sigma \subseteq C_{1}$ contém os isomorfismos de $C$ e é fechada por composição à esquerda e à direita pelos isomorfismos de $C$ (exemplos : as classes de todas as flechas de $C$ que são isomorfismos, epimorfismos; monomorfismos, epi regulares, mono regulares, inversíveis à direita, inversíveis à esquerda).

Proposição 1 : Nas condições acima temos:

(i) $\underset{\Xi}{\leftrightarrows}(C ;-)$ e $\underset{\Sigma}{\leftrightarrows}(C ;-)$ definem uma conexão de Galois entre as subclasses de $\operatorname{obj}(C A T)$ ; ordenadas por inclusão.

(ii)

$$
\begin{aligned}
& \mathbf{1} \in \underset{\Sigma}{\Sigma}(C, \mathcal{P}) . \\
& \mathbf{1} \in \underset{\Sigma}{L}(C, \mathcal{I}) .
\end{aligned}
$$

$\forall \mathcal{W} \subseteq \operatorname{obj}(C A T)(\underset{\Sigma}{\Sigma}(C, \mathcal{P}) \preceq \rightarrow \mathcal{W} \Rightarrow C$ é $\mathcal{W}$-ind completa $\wedge \mathcal{W} \subseteq \underline{\Sigma}(C, \mathcal{P}))$.

$\forall \mathcal{W} \subseteq \operatorname{obj}(C A T)(\underset{上}{\stackrel{\Sigma}{\leftrightarrows}}(C, \mathcal{I}) \preceq \leftarrow \mathcal{W} \Rightarrow C$ é $\mathcal{W}$-proj completa $\wedge \mathcal{W} \subseteq \underset{\rightleftarrows}{\rightleftarrows}(C, \mathcal{I}))$.

$\forall \mathcal{P}^{\prime} \in \operatorname{obj}(C A T)\left(\mathcal{P} \preceq \leftarrow \mathcal{P}^{\prime} \Rightarrow C\right.$ é $\mathcal{P}^{\prime}$-proj completa $\left.\wedge \underset{\Sigma_{(}}{\longrightarrow}(C, \mathcal{P}) \subseteq \underline{\Sigma}\left(C, \mathcal{P}^{\prime}\right)\right)$.

$\forall \mathcal{I}^{\prime} \in \operatorname{obj}(C A T)\left(\mathcal{I} \preceq \mathcal{I}^{\prime} \Rightarrow C\right.$ é $\mathcal{I}^{\prime}$-ind completa $\left.\wedge \underset{\longleftarrow}{\Sigma}(C, \mathcal{I}) \subseteq \sum \vec{\rightleftarrows}\left(C, \mathcal{I}^{\prime}\right)\right)$.

(v)

$C$ localmente pequena $\wedge C$ ind-completa (proj-completa) $\Rightarrow \forall \mathcal{W} \subseteq \operatorname{obj}(C A T)(\forall W \in$ $\mathcal{W} \exists I \in \underline{\Sigma}(C, \mathcal{P}) \exists T: W \rightarrow I(T$ final $\wedge T$ hom-bijetor $) \Rightarrow \mathcal{W} \subseteq \underline{\Sigma}(C, \mathcal{P}))$ ( pois $\left.\forall F: P \times W \rightarrow C F \stackrel{\cong}{\rightrightarrows} L_{\left(1_{P} \times T\right)}(F) \circ\left(1_{P} \times T\right)\left(F \stackrel{\cong}{\risingdotseq} R_{\left(1_{P} \times T\right)}(F) \circ\left(1_{P} \times T\right)\right)\right)$.

$C$ localmente pequena $\wedge C$ ind-completa (proj-completa) $\Rightarrow \forall \mathcal{W} \subseteq \operatorname{obj}(C A T)(\forall W \in$ $\mathcal{W} \exists P \in \Sigma(C, \mathcal{I}) \exists T: W \rightarrow P(T$ inicial $\wedge T$ hom-bijetor $) \Rightarrow \mathcal{W} \subseteq \Sigma(C: \mathcal{I}))($ pois 
$\left.\forall F: W \times I \rightarrow C F \stackrel{\cong}{\rightrightarrows} L_{\left(T \times 1_{I}\right)}(F) \circ\left(T \times 1_{I}\right)\left(F \stackrel{\cong}{\rightleftarrows} R_{\left(T \times 1_{I}\right)}(F) \circ\left(T \times 1_{I}\right)\right)\right)$.

(vi)

Se $C$ é ind-completa e composições à direita de flechas em $\Sigma$ por limites indutivos de flechas em $\Sigma$ estão em $\Sigma$ (exemplos : os isomorfismos, as inversiveis à direita, os epimorfismos) então : pela proposição (1.i) do ítem (1.3.1), a subcategoria plena de CAT associada a $\underline{\Sigma}(C, \mathcal{P})$ é fechada por limites indutivos indexados por $I \in \underline{\Xi}(C, \mathcal{P})$.

Se $C$ é proj-completa e composições à esquerda de flechas em $\Sigma$ por limites projetivos de flechas em $\Sigma$ estão em $\Sigma$ (exemplos : os isomorfismos, as inversíveis à esquerda, os monomorfismos) então : pela proposição (1.ii) do ítem (1.3.1); a subcategoria plena de $C A T$ associada a $\sum(C, \mathcal{I})$ é fechada por limites indutivos indexados por $P^{\star}$ onde $P \in \underset{\longleftarrow}{\longleftarrow}(C, \mathcal{I})$.

(vii)

Dado $\Gamma: X \rightarrow C A T$ se $C$ é proj-completa e $I \in I \underset{S O}{\longrightarrow}\left(C:\left\{X^{\star}\right\} \cup\left\{\Gamma(x): x \in X_{0}\right\}\right)$ então $I \in I \stackrel{\operatorname{SO}}{\rightarrow}(C:\{\underset{X}{\longrightarrow} \Gamma\})$.

Dado $\Gamma: \vec{X} \rightarrow C A T$ se $C$ é ind-completa e $P \in I S O\left(C:\{X\} \cup\left\{\Gamma(x): x \in X_{0}\right\}\right)$ então $P \in I S O\left(C:\left\{\lim _{\longleftrightarrow} \Gamma\right\}\right)$.

(viii)

$C$ proj-completa $\Rightarrow I S O(C:\{P\}) \subseteq I S O\left(C:\left\{\mathcal{D}\left(\pi_{0}(P)\right)\right\}\right)$.

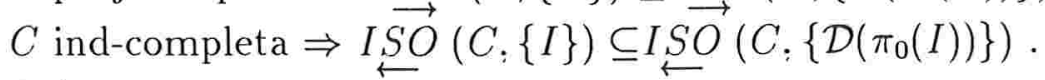

(ix)

$\underset{\longrightarrow S O}{\longrightarrow}(S E T,\{P\})=\underset{I S O}{\longrightarrow}\left(S E T:\left\{\mathcal{D}\left(\pi_{0}(P)\right)\right\} \cup \pi_{0}(P)\right)$.

$\overrightarrow{I S O}(S E T ;\{I\})=\underset{\leftarrow}{\stackrel{S O}{\longleftarrow}}\left(S E T ;\left\{\mathcal{D}\left(\pi_{0}(I)\right)\right\} \cup \pi_{0}(I)\right)$.

Observação 3 : Podemos relativizar as definições e resultados acima, isto é, se $\mathcal{P} \subseteq$ $(U-C A T)_{0}\left(\mathcal{I} \subseteq(U-C A T)_{0}\right)$ e $C$ é $\mathcal{P}$-proj completa $(\mathcal{I}$-ind completa) então a restrição

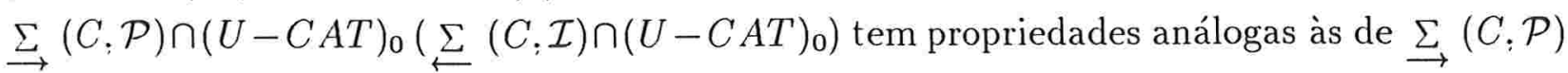
$\left.\overrightarrow{\left(\sum\right.}(C, \mathcal{I})\right)$, relativizadas a $U \in U N I V$.

Proposição 2: $\forall P \in \operatorname{obj}(C A T)\left(\underset{\operatorname{EPI}}{\longrightarrow}(S E T:\{P\}) \subseteq\left\{I \in \operatorname{obj}(C A T): \forall F: P^{\star} \rightarrow I\right.\right.$ $\left.\exists i \in I_{0}\left(N A T\left(F, \Delta_{P \star}(i)\right) \neq \emptyset\right)\right\}$.

Dado $F: P^{\star} \rightarrow I$ seja $F^{\prime}=I\left(F\left({ }_{-}\right),-\right): P \times I \rightarrow S E T$.

$\# \underset{P}{\lim } \underset{I}{\lim } F^{\prime}(p, i)=1$.

$P=\emptyset$ ok.

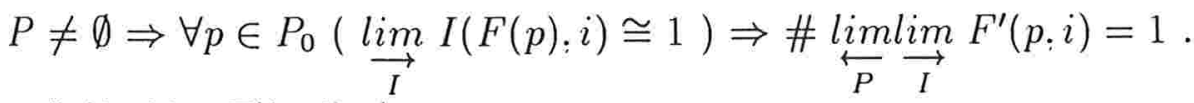

. \# $\underset{I}{\lim } \underset{P}{\stackrel{i m}{i m}} F^{\prime}(p, i) \neq 0$

Pelo ítem anterior e porque can $\left(F^{\prime}\right)$ é sobrejetora.

. $\exists i \in I_{0}\left(N A T\left(F: \Delta_{P \star}(i)\right) \neq \emptyset\right)$. 
Pelo ítem anterior temos $I \neq \emptyset$ e $\exists i \in I_{0}\left(\emptyset \neq \stackrel{\lim }{P}_{P} I(F(p), i) \cong N A T\left(F \cdot \Delta_{P \star}(i)\right)\right)$.

\section{Definição 2 :}

(i) Sejam $G$ grafo pequeno e $A$ categoria. A é categoria $G$-filtrada $\Longleftrightarrow \forall H: G \rightarrow \mathcal{E}(A)$ $(H-$ contindcom $\neq \emptyset$ ) .

(ii) Sejam $C$ categoria pequena e $A$ categoria. A é categoria $C$-filtrada $\Longleftrightarrow \forall F: C \rightarrow A$ $(F-$ coneindcom $\neq \emptyset$ ).

Observação 4 :

(i) $A$ é $G$-filtrada $\Longleftrightarrow A$ é $\mathcal{L}(G)$-filtrada.

(ii) $A$ é $\mathcal{E}(C)$-filtrada $\Rightarrow A$ é $C$-filtrada.

Definição 3 : Sejam $\kappa$ cardinal infinito e $A$ categoria. $A$ é categoria $\kappa$-filtrada $\Longleftrightarrow$ $\forall G \in \operatorname{obj}(G R A)(\# G<\kappa \Rightarrow A$ é G-filtrada ) .

Proposição 3 :

(i) $A$ é $\kappa$-filtrada $\Longleftrightarrow \forall C \in \operatorname{obj}(C A T)(\# C<\kappa \Rightarrow A$ é $C$-filtrada ). (Ter cautela na demonstração de $(\Leftarrow)$ para $\kappa=\omega$ !)

(ii) $A$ é $\kappa$-filtrada $\Longleftrightarrow\left(\forall 0 \leq \lambda<\kappa\left(A\right.\right.$ é $D_{\lambda}$-filtrada $) \wedge \forall 0<\sigma<\kappa$ ( $A$ é $P_{\sigma}$-filtrada ) ) onde $D_{\lambda}$ é o (único a menos de isomorfismo) grafo discreto com $\lambda$ objetos ( $\# \operatorname{mor}\left(D_{\lambda}\right)=0$; \#obj $\left(D_{\lambda}\right)=\lambda$ ) e $P_{\sigma}$ é o (único a menos de isomorfismo) grafo com $\sigma$ flechas paralelas $\left(\# \circ b j\left(P_{\sigma}\right)=2 ; \# P_{\sigma}(0,1)=\sigma\right)$.

(iii) $A$ é $D_{0}$-filtrada $(\Longleftrightarrow A \neq \emptyset) \wedge A$ é $D_{2}$-filtrada $\Rightarrow \# \pi_{0}(A)=1$.

(iv) $A$ é $\omega$-filtrada $\Longleftrightarrow$ ( $A$ é $D_{0}$-filtrada $\wedge A$ é $D_{2}$-filtrada $\wedge A$ é $P_{2}$-filtrada ).

(v) $\forall \omega \leq \kappa_{0} \leq \kappa_{1}\left(A \kappa_{1^{-}}\right.$filtrada $\Rightarrow A \kappa_{0}$-filtrada $)$.

(vi) $A$ é $\kappa$-filtrada $\Longleftrightarrow A$ é $r \epsilon g(\kappa)$-filtrada onde $r \epsilon g(\kappa)=\min \{\lambda: \kappa \leq \lambda \wedge \lambda$ é regular $\}$ $\left(r \in g(\kappa) \in\left\{\kappa, \kappa^{+}\right\}\right)$.

(vii) $\forall U(U \in U N I V \vee U=V) \forall \kappa(\omega \leq \kappa=r \epsilon g(\kappa) \in U)$ sejam $\mathcal{X}_{\kappa}=\{X \in$ $(U-C A T)_{0}: X$ é categoria associada a ordem $\kappa$-dirigida $\} . \mathcal{Y}_{\kappa}=\left\{Y \in(U-C A T)_{0}: Y\right.$ é categoria associada a pré-ordem $\kappa$ - dirigida $\}, \mathcal{Z}_{\kappa}=\left\{Z \in(U-C A T)_{0}: Z\right.$ é categoria $\kappa$-filtrada então $\mathcal{Y}_{\kappa} \preceq \rightarrow \mathcal{X}_{\kappa} \preceq \rightarrow \mathcal{Z}_{\kappa} \preceq \rightarrow \mathcal{Y}_{\kappa}$. (O ítem não trivial $\left(\mathcal{X}_{\kappa} \preceq \rightarrow \mathcal{Z}_{\kappa}\right.$ ) segue adaptando-se a demonstração da proposição 8.1 .6 em [098] $(\omega \sim \kappa)$.)

(viii) Uma categoria $B$ é $U$-ind completa $\Longleftrightarrow \exists \omega \leq \kappa=r \epsilon g(\kappa)<\# U$ ( $B$ é $(<\kappa)$-ind completa $\wedge B$ é $\mathcal{Z}_{\kappa}$-ind completa ).

Proposição 4 : Seja $\kappa$ cardinal regular infinito. Em SET, limites indutivos pequenos $\kappa$-filtrados comutam com limites projetivos (pequenos) $<\kappa$-indexados.

Como $\left\{X \in(C A T)_{0}: X\right.$ é categoria associada a ordem $\kappa$-dirigida $\} \preceq\left\{Z \in(C A T)_{0}: Z\right.$ é categoria $\kappa$ - filtrada $\}$ basta mostrar que $\left\{I \in(C A T)_{0}: I\right.$ é categoria associada a ordem 
$\kappa$-dirigida $\} \subseteq \underset{\subseteq S O}{\longrightarrow}\left(S E T .\left\{P \in(C A T)_{0}: \# P<\kappa\right\}\right)$. Seja $F: P \times I \rightarrow S E T$ então $x \underset{\stackrel{\text { im }}{\stackrel{\lim }{\longrightarrow}} F(p, i)}{\longrightarrow} \Longleftrightarrow\left(x=\left(x_{p}\right)_{p \in P_{0}} \wedge \forall p \in P_{0} \exists i_{p} \in I_{0} \exists y_{p} \in F\left(p, i_{p}\right)\left(x_{p}=\left[\left(i_{p}, y_{p}\right)\right]\right) \wedge\right.$ $\left.\left.\forall f: p \rightarrow p^{\prime}\left(\underset{I}{\left(\lim _{\longrightarrow}\right.} F(f, i)\right)\left(x_{p}\right)=x_{p^{\prime}}\right)\right)$. Logo como $\# P_{0} \leq \# P<\kappa$ e $I$ é ordem $\kappa$-dirigida $\exists i \in I_{0} \exists\left(z_{p}\right)_{p \in P_{0}}^{I}\left(=\left(F\left(1_{p}, i_{p} \rightarrow i\right)\left(y_{p}\right)\right)_{p \in P_{0}}\right) \in \prod_{p \in P_{0}} F(p, i)\left(\left(x_{p}\right)_{p \in P_{0}}=\left(\left[\left(i, z_{p}\right)\right]\right)_{p \in P_{0}}\right.$ $\left.\wedge \forall f: p \rightarrow p^{\prime}\left(\left[\left(i, F\left(f, 1_{i}\right)\left(z_{p}\right)\right)\right]=\left[\left(i, z_{p^{\prime}}\right)\right]\right)\right) \Rightarrow \forall f: p \rightarrow p^{\prime} \exists i_{f} \in I_{0}\left(F\left(1_{p^{\prime}} ; i \rightarrow\right.\right.$ $\left.\left.i_{f}\right) \circ F\left(f, 1_{i}\right)\left(z_{p}\right)=F\left(1_{p^{\prime}} ; i \rightarrow i_{f}\right)\left(z_{p^{\prime}}\right)\right) \Rightarrow \forall f: p \rightarrow p^{\prime} \exists i_{f} \in I_{0^{\prime}}\left(w_{(p, f)}=F\left(1_{p} ; i \rightarrow i_{f}\right)\left(z_{p}\right) \in\right.$ $F\left(p, i_{f}\right) \wedge w_{\left(f: p^{\prime}\right)}=F\left(1_{p^{\prime}}, i \rightarrow i_{f}\right)\left(z_{p^{\prime}}\right) \in F\left(p^{\prime}, i_{f}\right) \Rightarrow\left(x_{p}=\left[\left(i_{f} ; w_{(p, f)}\right)\right] \wedge x_{p^{\prime}}=\left[\left(i_{f} ; w_{\left(f ; p^{\prime}\right)}\right)\right]\right.$ $\left.\left.\wedge F\left(f, 1_{i_{f}}\right)\left(w_{(p, f)}\right)=w_{\left(f, p^{\prime}\right)}\right)\right)$. Logo como \# $P_{1} \leq \# P<\kappa$ e I é categoria associada a ordem $\kappa$-dirigida $\exists i_{x} \in I_{0} \exists\left(u_{p}\right)_{p \in P_{0}}\left(=\left(F\left(1_{p} ; i \rightarrow i_{f} \rightarrow i_{x}\right)\left(z_{p}\right)\right)_{p \in P_{0}}\right) \in \prod_{p \in P_{0}} F\left(p ; i_{x}\right)($ $\left.\left(x_{p}\right)_{p \in P_{0}}=\left(\left[\left(i_{x}, u_{p}\right)\right]\right)_{p \in P_{0}} \wedge \forall f: p \rightarrow p^{\prime}\left(F\left(f, 1_{i_{x}}\right)\left(u_{p}\right)=u_{p^{\prime}}\right)\right) .^{4} \operatorname{Seja}\left(j:\left(v_{p}\right)_{p \in P_{0}}\right)$ tal que $j \in I_{0} \wedge \forall p \in P_{0}\left(v_{p} \in F(p, j) \wedge x_{p}=\left[\left(j, v_{p}\right)\right]\right) \wedge \forall f: p \rightarrow p^{\prime}\left(F\left(f ; 1_{j}\right)\left(v_{p}\right)=v_{p^{\prime}}\right)$ então; como $I$ é $\kappa$-dirigida $(\mathrm{e} 2<\kappa), \forall p \in P_{0} \exists k_{p} \in I_{0}\left(F\left(1_{p}, i_{x} \rightarrow k_{p}\right)\left(u_{p}\right)=F\left(1_{p} ; j \rightarrow k_{p}\right)\left(v_{p}\right)\right)$. Como \#P $\leq$ \#P $<\kappa$ e $I$ é $\kappa$-dirigida $\exists k \in I_{0} \exists\left(t_{p}\right)_{p \in P_{0}}\left(=\left(F\left(1_{p}, i_{x} \rightarrow k_{p} \rightarrow k\right)\left(u_{p}\right)\right)_{p \in P_{0}}=\right.$ $\left.\left(F\left(1_{p}, j \rightarrow \kappa_{p} \rightarrow k\right)\left(v_{p}\right)\right)_{p \in P_{0}}\right) \in \prod_{p \in P_{0}} F(p, k)\left(\left(x_{p}\right)_{p \in P_{0}}=\left(\left[\left(k, t_{p}\right)\right]\right)_{p \in P_{0}} \wedge \forall f: p \rightarrow p^{\prime}(\right.$

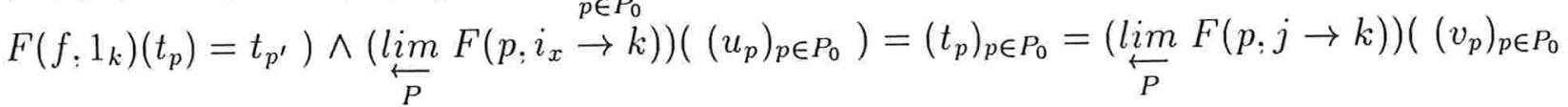
)) $\cdot^{5}$

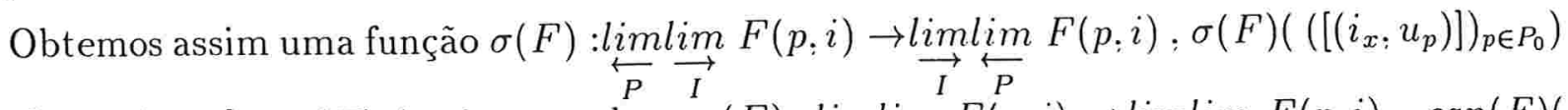
)$=\left[i_{x},\left(u_{p}\right)_{p \in P_{0}}\right] \cdot \sigma(F)$ é a inversa de $\operatorname{can}(F) \underset{I}{: \lim _{P}} \underset{P}{\lim } F(p, i) \rightarrow \underset{P}{\stackrel{\lim }{\longrightarrow} \underset{I}{\longrightarrow}} F(p, i), \operatorname{can}(F)($ $\left.\left[\left(i,\left(r_{p}\right)_{p \in P_{0}}\right)\right]\right)=\left(\left[i, r_{p}\right]\right)_{p \in P_{0}}$.

Observação $4:$ Se $\kappa \in U \in U N I V$ podemos substituir $S E T \leadsto U-S E T$ e reinterpretar "pequeno": $\in(C A T)_{0} \rightsquigarrow \in(U-C A T)_{0}$.

Proposição 5 : Dado $\kappa$ cardinal regular infinito temos $\underset{S O}{\longrightarrow}\left(S E T:\left\{P \in(C A T)_{0}\right.\right.$ : $\# P<\kappa\})=\left\{I \in(C A T)_{0}: I\right.$ é $\kappa$-filtrada $\}$.

$. \subseteq: \underset{\longrightarrow}{\operatorname{SSO}}\left(S E T:\left\{P \in(C A T)_{0}: \# P<\kappa\right\}\right) \underset{\text { prop.(1.i) }}{=} \cap\left\{\stackrel{\operatorname{ISO}}{\longrightarrow}(S E T,\{P\}): P \in(C A T)_{0}\right.$ $\wedge \# P<\kappa\} \subseteq \cap\left\{\underset{\operatorname{EPI}}{\longrightarrow}(S E T,\{P\}): P \in(C A T)_{0} \wedge \# P<\kappa\right\} \underset{\text { prop. } 2}{\subseteq} \cap\left\{\left\{I \in(C A T)_{0}: I\right.\right.$ é $P^{\star}$-filtrada $\left.\}: P \in(C A T)_{0} \wedge \# P<\kappa\right\}=\left\{I \in(C A T)_{0}: I\right.$ é $\kappa$-filtrada $\}$.

. $\supseteq$ : proposição 4 .

Observação 5: $\kappa \in U \in U N I V \Rightarrow I \underset{S O}{\longrightarrow}\left(U-S E T:\left\{P \in(U-C A T)_{0}: \# P<\right.\right.$

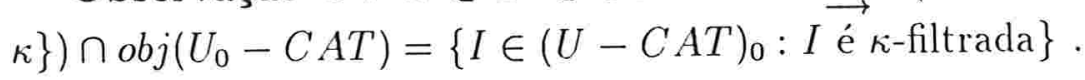

${ }^{4}$ Aqui utilizamos a hipótese que há no máximo uma flecha entre dois objetos.

${ }^{5}$ Idem. 


\section{Definição 4 :}

(i) Seja $C$ categoria. $C$ é (ind)-degenerada $\Longleftrightarrow 1_{C}$-contindcom $\neq \emptyset$.

(ii) Denotamos $\mathrm{E}$ a (única a menos de isomorfismo) categoria $\operatorname{com} \# o b j(\mathrm{E})=1 \mathrm{e}$ $\# \operatorname{mor}(\mathbf{E})=2$ (E é a "menor categoria com idempotente não trivial").

(iii) Sejam $C$ categoria e $\epsilon \in C_{1}$ um idempotente de $C$ (i.e. $\exists c \in \operatorname{obj}(C)(\epsilon \in C(c, c) \wedge$ $\epsilon \circ \epsilon=\epsilon))$. Denotamos $C I S(\epsilon)=\left\{(p, i): \exists a \in C_{0}(p \in C(a, c) \wedge i \in C(c ; a) \wedge p \circ i=\epsilon\right.$ $\left.\left.\wedge i \circ p=1_{a}\right)\right\}$ o conjunto das cisões de $\epsilon$. Dizemos que uma categoria é Karoubiana quando todos seus idempotentes se cindem.

\section{Proposição 6 :}

(i) $\mathbf{E}=\mathbf{E}^{\star}$.

(ii) $\forall U \in U N I V$ ( $\mathbf{E}$ é categoria praticamente em $U$ ).

(iii) $\forall X \in(C A T)_{0}$ ( $X$ é E-filtrada $\wedge \mathbf{E}$ é $X$-filtrada ).

(iv) $\forall X \in(C A T)_{0}\left(X\right.$ degenerada $\left.\Rightarrow \pi_{0}(X) \cong 1\right)$.

(v) $\forall X \in(C A T)_{0}$ ( $X$ degenerada $\Longleftrightarrow \exists F: \mathbf{E} \rightarrow X$ final $)$.

(vi) Sejam $C$ categoria, $F: \mathbf{E} \rightarrow C$ funtor e $\epsilon \in C(c, c)$ o idempotente de $C$ determinado por $F$ então:

- $\forall a \in C_{0} \forall p \in C(a, c)$ ( $p$ determina um cone projetivo limite de base $F \Longleftrightarrow$ $\exists i \in C(c, a)((p, i) \in C I S(\epsilon)))$.

. $\forall a \in C_{0} \forall i \in C(c, a)$ ( $i$ determina um cone indutivo limite de base $F \Longleftrightarrow \exists p \in C(a, c)$ $((p, i) \in C I S(\epsilon)))$.

. Como todo funtor preserva cisões de idempotentes, todo funtor preserva limites projetivos/indutivos de tipo $\mathbf{E}$.

Proposição $7: I S O S\left(S E T,(C A T)_{0}\right)=\left\{I \in(C A T)_{0}: I\right.$ é degenerada $\}=\{\mathbf{E}\} \preceq \rightarrow$ $=\left\{I \in(C A T)_{0}: \exists F: \overrightarrow{\mathbf{E}} \rightarrow I\right.$ final $\left.\}\right)$.

. $\supseteq$ : Como limites projetivos comutam com limites projetivos e E-limites projetivos correspondem a $\mathbf{E}$-limites indutivos temos $\mathbf{E} \in I \underset{S O}{\longrightarrow}\left(S E T,(C A T)_{0}\right)$. Mas $\{\mathbf{E}\} \subseteq I S O$ $\left(S E T ;(C A T)_{0}\right) \Rightarrow\{\mathbf{E}\}^{\preceq^{\rightarrow}} \subseteq \underset{I S O}{\longrightarrow}\left(S E T ;(C A T)_{0}\right)$.

$. \subseteq: I S O\left(S E T,(C A T)_{0}\right) \subseteq \underset{\longrightarrow}{\operatorname{EP} I}\left(S E T,(C A T)_{0}\right) \subseteq\left\{I \in(C A T)_{0}: \forall P \in(C A T)_{0}(I\right.$ é $P^{*}$-filtrada) $\vec{\longrightarrow} \subseteq\left\{I \in(C A T)_{0}: I\right.$ é degenerada $\}=\{\mathbf{E}\} \preceq^{-}$.

Proposição 8: Em SET , limites indutivos indexados por coprodutos pequenos de categorias degeneradas e pequenas comutam com limites projetivos conexos, não vazios e pequenos.

Pelas proposições (1.vii) e 7, basta demonstrar o seguinte caso: 
Corolário : Em SET. coprodutos pequenos comutam com limites projetivos conexos; não vazios e pequenos.

Sejam $P: I \in(C A T)_{0}$ tais que $\pi_{0}(P) \cong 1$ e $I \cong \mathcal{D}\left(I_{0}\right)$. Seja $F: P \times I \rightarrow S E T$.

$I=\emptyset$

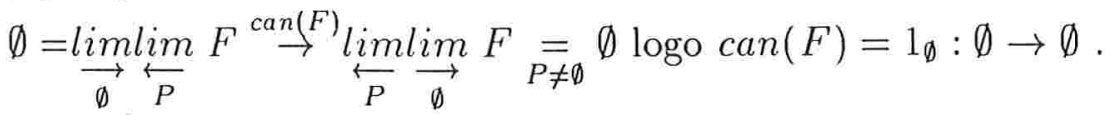

$. I \neq \emptyset$

$x \underset{P}{\in \lim _{I}} \underset{I}{\longrightarrow} F(p ; i) \Longleftrightarrow x=\left(x_{p}\right)_{p \in P_{0}} \wedge \forall p \in P_{0} \exists i_{p} \in I_{0} \exists y_{p} \in F\left(p, i_{p}\right)\left(x_{p}=\left(i_{p}, y_{p}\right)\right) \wedge$ $\forall f: p \rightarrow p^{\prime}\left(\left(i^{\prime}, F\left(f, 1_{i^{\prime}}\right)\left(y_{p}\right)\right)=\underset{I}{\lim } F(f ; i)\left(x_{p}\right)=x_{p^{\prime}}=\left(i_{p^{\prime}}, y_{p^{\prime}}\right)\right)$. Logo como $P$ é conexa e não vazia temos bijeções : $\left\{x=\left(x_{p}\right)_{p \in P_{0}}: x \underset{P}{\in \underset{I}{\lim } \underset{I}{\longrightarrow}} F(p, i)\right\} \cong\left\{y=\left(i, y_{p}\right)_{p \in P_{0}}: i \in I_{0} \wedge\right.$ $\left.\forall p \in P_{0}\left(y_{p} \in F(p, i)\right) \wedge \forall f: p \rightarrow p^{\prime}\left(F\left(f, 1_{i^{\prime}}\right)\left(y_{p}\right)=y_{p^{\prime}}\right)\right\} \cong\left\{z=\left(i ;\left(z_{p}\right)_{p \in P_{0}}\right): i \in I_{0} \wedge\right.$

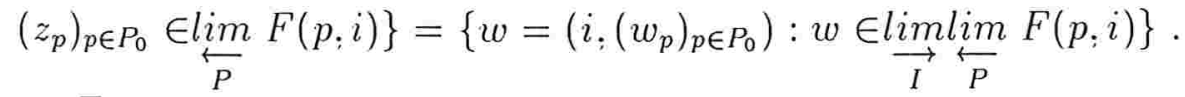

Proposição $9: \operatorname{ISO}\left(S E T,\left\{P \in(C A T)_{0}: \# \pi_{0}(P)=1\right\}\right)=\left\{I \in(C A T)_{0}: \exists X \in\right.$ $\left.(S E T)_{0} \exists\left\{I_{x} \in(C A T)_{0}: I_{x} \text { é degenerada }\right\}_{x \in X}\left(I=\coprod_{x \in X} I_{x}\right)\right\}$.

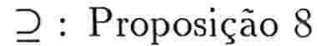

$\subseteq: \underset{\longrightarrow S O}{\longrightarrow}\left(S E T,\left\{P \in(C A T)_{0}: \# \pi_{0}(P)=1\right\}\right) \subseteq \underset{\longrightarrow}{\longrightarrow} I\left(S E T,\left\{P \in(C A T)_{0}: \# \pi_{0}(P)=\right.\right.$ $1\}) \subseteq\left\{\overrightarrow{I \in}(C A T)_{0}: I\right.$ é $P^{\star}$-filtrada $\left.\wedge \# \pi_{0}(P)=1\right\} \subseteq\left\{I \in(C A T)_{0}: \forall I^{\prime} \in \pi_{0}(I)\left(I^{\prime}\right.\right.$ é degenerada $)\}=\left\{I \in(C A T)_{0}: \exists X \in(S E T)_{0} \exists\left\{I_{x} \in(C A T)_{0}: I_{x} \text { é degenerada }\right\}_{x \in X}\right.$ $\left.\left(I=\coprod_{x \in X} I_{x}\right)\right\}$.(Notemos que toda categoria pequena se decompoẽ "univocamente" como coproduto pequeno de categorias pequenas conexas e não vazias.)

\section{Observação 6 :}

(i) Seja $\mathcal{I} \subseteq\left\{I \in(C A T)_{0}: I\right.$ é coproduto de categorias degeneradas $\}$ e tal que $\mathcal{D}(2) \in \mathcal{I}$ então $\operatorname{ISO}(S E T, \mathcal{I})=\left\{P \in(C A T)_{0}: \# \pi_{0}(P)=1\right\}$.

(ii) Como em SET (com o axioma da escolha!) as noções de flecha inversível à direita, epimorfismo regular e epimorfismo coincidem com a de função sobrejetora, temos que, em $S E T$; produtos pequenos comutam com coigualadores de duas flechas paralelas .

\subsubsection{Completamentos indutivos}

Referências adicionais : [092] : [207] : [195] .

Nesta seção fixamos três universos distintos e arbitrários : $U_{0} \in U_{1} \in U_{2} \in V$. 
Definição $1:$ Sejam $\mathcal{I} \subseteq$ obj $\left(U_{0}-C A T\right)$ e $A \in \operatorname{obj}\left(U_{1}-C A T\right)$. Denominamos objetos $\mathcal{I}$-presentáveis os elementos de $A_{\mathcal{I}}=\left\{a \in A_{0}: A(a:-): A \rightarrow S E T\right.$ preserva $\mathcal{I}$-limites indutivos $\}$.

\section{Observação 1 :}

(i) Não utilizaremos distinção notacional entre o conjunto $A_{\mathcal{I}}$ e a subcategoria plena de $A$ de seus objetos $\mathcal{I}$-presentáveis.

(ii) Considerar as "restrições" dos funtores $A\left(a_{;--}\right)$a $U_{1}-S E T$ ( ou $U_{0}-S E T$ caso $A$ esteja praticamente localmente em $\left.U_{0}\right)$ não altera $A_{\mathcal{I}}$ e suas propriedades.

(iii) (A subategoria plena de $A$ com objetos) $A_{\mathcal{I}}$ é fechada em $A$ por isomorfismos; por retrações , por limites indutivos de tipo $\underset{S O}{S O}(S E T, \mathcal{I})$ e de modo geral, por $U_{0}$ - diagramas multiplamente limites indutivos de tipo $\underset{I S O}{\longrightarrow}(S E T, \mathcal{I})$.

Definição 2: Sejam $\mathcal{I} \subseteq$ obj $\left(U_{0}-C \overrightarrow{A T}\right)$ e $C \in \operatorname{obj}\left(U_{1}-C A T\right)$. Um $\mathcal{I}$-ind completamento forte de $C$ é um funtor $J \in U_{1}-C A T(C, A)$ tal que:

. A é $\mathcal{I}$-ind completa ;

. $J$ é hom-bijetor ;

. $\forall a \in A_{0}\left(\exists c \in C_{0}(a \cong J(c)) \vee(\exists I \in \mathcal{I} \exists H: I \rightarrow C(a \cong \underset{I}{\lim } J \circ H))\right)$;

. $\left(J_{0}\right)_{*}\left(C_{0}\right) \subseteq A_{\mathcal{I}}$.

\section{Observação 2 :}

(i) $A$ é o fecho pleno e repleto de $\left(J_{0}\right)_{*}\left(C_{0}\right)$ por $\mathcal{I}$-limites indutivos, isto é, se $i: \bar{A} \hookrightarrow A$ é hom-bijetor, cria isomorfismos e $\mathcal{I}$-limites indutivos e $\left(J_{0}\right)_{*}\left(C_{0}\right) \subseteq \bar{A}_{0}$ então $\bar{A}=A$ ( notação $: A=\left[\left(J_{0}\right)_{*}\left(C_{0}\right)\right]^{\mathcal{I}-\lim } \longrightarrow$ ).

(ii) Seja $J^{\prime} \in U_{1}-C A T\left(C^{\prime} ; A\right)$ tal que $\exists T: C^{\prime} \cong C\left(J^{\prime} \cong J \circ T\right)$ então $J^{\prime}$ é $\mathcal{I}$-ind completamento forte de $C^{\prime}$.

(iii) Seja $J^{\prime} \in U_{1}-C A T\left(C, A^{\prime}\right)$ tal que $\exists T: A \cong A^{\prime}\left(J^{\prime} \cong T \circ J\right)$ então $J^{\prime}$ é $\mathcal{I}$-ind completamento forte de $C$.

(iv) Seja $B$ subcategoria plena de $A \operatorname{com}\left(J_{0}\right)_{*}\left(C_{0}\right) \subseteq B_{0} \subseteq A_{\mathcal{I}}$ então $i: B \hookrightarrow A$ é $\mathcal{I}$-ind completamento forte de $B$.

(v) $\tilde{J}: A \rightarrow\left[C^{*}, S E T\right]$ preserva $(C A T)_{0^{-}}$-limites projetivos e $\mathcal{I}$-limites indutivos ; sua restrição a $\left[C^{\star}, U_{1}-S E T\right]$ preserva $\left(U_{1}-C A T\right)_{0}$-limites projetivos e $\mathcal{I}$-limites indutivos . Quando $A$ está praticamente localmente em $U_{0}$ a restrição (a menos de isomorfismo) de $\tilde{J}$ a $\left[C^{\star}, U_{0}-S E T\right]$ preserva $\left(U_{0}-C A T\right)_{0}$-limites projetivos e $\mathcal{I}$-limites indutivos.

(vi) $\forall a, a^{\prime} \in A_{0}$ temos:

. $a \cong J(c) \wedge a^{\prime} \cong J\left(c^{\prime}\right) \Rightarrow A\left(a, a^{\prime}\right) \cong C\left(c, c^{\prime}\right)$.

. $a \cong \underset{I}{\lim } J \circ H \wedge a^{\prime} \cong J\left(c^{\prime}\right) \Rightarrow A\left(a, a^{\prime}\right) \cong \lim _{I^{\star}} C(H(i), c)$

. $a \cong J(c) \wedge a^{\prime} \cong \underset{I^{\prime}}{\lim } J \circ H^{\prime} \Rightarrow A\left(a ; a^{\prime}\right) \cong \underset{I^{\prime}}{\stackrel{\lim }{\longrightarrow}} C\left(c ; H^{\prime}\left(i^{\prime}\right)\right)$. 
. $a \cong \underset{I}{\lim } J \circ H \wedge a^{\prime} \cong \underset{I^{\prime}}{\lim } J \circ H^{\prime} \Rightarrow A\left(a, a^{\prime}\right) \cong \underset{I^{*}}{\lim } \underset{I^{\prime}}{\lim } \lim \left(H(i) \cdot H^{\prime}\left(i^{\prime}\right)\right)$.

(vii) $C$ está praticamente localmente em $U_{0} \Rightarrow$ ( $A$ está praticamente localmente em $U_{0}$ $\wedge \forall a \in A_{0}\left((J \rightarrow a)\right.$ está praticamente em $\left.\left.U_{0}\right)\right)$.

(viii) $A_{\mathcal{I}}=\left[\left(J_{0}\right)_{*}\left(C_{0}\right)\right] \stackrel{\{\mathbf{E}\}-\lim }{\rightarrow}=\left\{a \in A_{0}: \exists c \in C_{0} \exists \epsilon: J(c) \rightarrow J(c) \exists p: a \rightarrow J(c)\right.$ $\left.\exists i: J(c) \rightarrow a\left(\epsilon \circ \epsilon=\epsilon \wedge p \circ i=\epsilon \wedge 1_{a}=i \circ p\right)\right\}$ ( logo: $C U_{1^{-}}$categoria essencialmente em $U_{0} \Rightarrow A_{\mathcal{I}} U_{1}$-categoria essencialmente em $\left.U_{0}\right)$.

(ix) $\mathcal{I}-\{\emptyset\} \neq \emptyset \Rightarrow \forall a \in A_{0} \exists I \in \mathcal{I} \exists H: I \rightarrow C(a \cong \underset{I}{\lim } J \circ H)$.

(x) $\mathcal{I}=\emptyset \Rightarrow 1_{C}: C \rightarrow C$ é $\mathcal{I}$-ind completamento forte de $C \in o b j\left(U_{1}-C A T\right)$.

(xi) $\mathcal{I}=\{\emptyset\} \Rightarrow J: C \rightarrow C^{-}$é $\mathcal{I}$-ind completamento forte de $C \in \operatorname{obj}\left(U_{1}-C A T\right)$.

Definição 3: Sejam $\mathcal{I} \subseteq o b j\left(U_{0}-C A T\right)$ e $C \in \operatorname{obj}\left(U_{1}-C A T\right)$. Um $\mathcal{I}$-ind completamento fraco de $C$ é um funtor $J \in U_{1}-C A T(C, A)$ tal que:

. $A$ é $\mathcal{I}$-ind completa;

. $\forall B U_{1}$-categoria $\mathcal{I}$-ind completa $\forall F: C \rightarrow B \exists L_{J}(F) \wedge L_{J}(F)$ é pontual;

. $\forall B U_{1}$-categoria $\mathcal{I}$-ind completa a adjunção $\left(L_{J},[J, B], \eta, \epsilon\right):[C, B] \rightarrow[A, B]$ se restringe a uma equivalência de adjunção : $[C, B] \stackrel{\Im}{\rightarrow}[A, B]_{\mathcal{I} \text {-ind.pres. }}$ onde $[A, B]_{\mathcal{I} \text {-ind.pres. }}$ é a subcategoria plena de $[A, B]$ dos funtores que preservam $\mathcal{I}$-limites indutivos.

\section{Observação 3 :}

(i) Seja $J^{\prime} \in U_{1}-C A T\left(C^{\prime}: A\right)$ tal que $\exists T: C^{\prime} \cong C\left(J^{\prime} \cong J \circ T\right)$ então $J^{\prime}$ é $\mathcal{I}$-ind completamento fraco de $C^{\prime}$.

(ii) Seja $J^{\prime} \in U_{1}-C A T\left(C, A^{\prime}\right)$ tal que $\exists T: A \cong A^{\prime}\left(J^{\prime} \cong T \circ J\right)$ então $J^{\prime}$ é $\mathcal{I}$-ind completamento fraco de $C$.

(iii) Seja $J^{\prime} \in U_{1}-C A T\left(C, A^{\prime}\right)$ tal que $J^{\prime}$ é $\mathcal{I}$-ind completamento fraco de $C$ então $\exists T: A \cong A^{\prime}\left(J^{\prime} \cong T \circ J\right)$.

(iv) $\mathcal{I} \in\{\emptyset ;\{\emptyset\}\} \Rightarrow \forall J \in U_{1}-C A T(C, A)(J$ é $\mathcal{I}$-ind completamento forte de $C \Rightarrow J$ é $\mathcal{I}$-ind completamento fraco de $C$ ).

Sejam $J \in U_{1}-C A T(C, A)$ e $J^{\rightarrow}: A \rightarrow\left(\left(U_{1}-C A T\right) \rightarrow C\right)$ como no ítem (1.2.3). Dado $H \in U_{1}-C A T(I, C)$ temos isomorfismo natural $[I, A]\left(J \circ H, \Delta_{I}(-)\right) \cong\left(\left(U_{1}-C A T\right) \rightarrow\right.$ $C)(H, J \rightarrow(-)), \log \circ \forall a \in A_{0} \forall I \in o b j\left(U_{1}-C A T\right)$ temos bijeções inversas $\phi(a, I): X(a, I) \rightleftarrows$ $Z(a, I): \psi(a, I)$ onde $X(a, I)=\left\{(H, \mu): H \in U_{1}-C A T(I, C) \wedge \mu \in[I, A]\left(J \circ H, \Delta_{I}(a)\right)\right.$ \},$Z(a, I)=U_{1}-C A T(I,(J \rightarrow a))$.

Dados $a \in A_{0}, I \in \operatorname{obj}\left(U_{1}-C A T\right),(H, \mu) \in X(a, I)$ consideremos para cada $c \in C_{0}$ a função $\pi_{0}(c \rightarrow H) \stackrel{\Gamma_{\varsigma}}{\rightarrow} A(J(c), a)=$

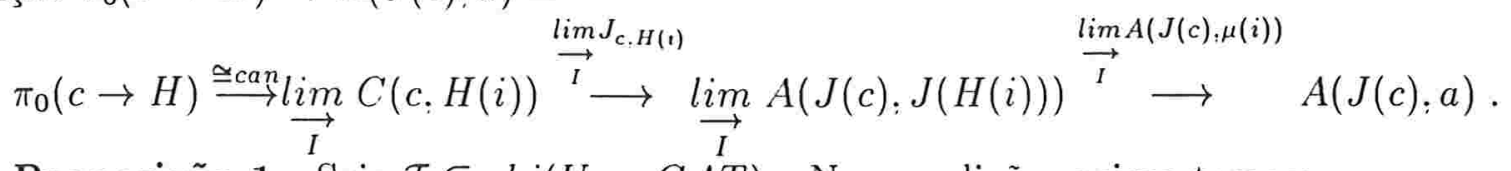

Proposição 1 : Seja $\mathcal{I} \subseteq o b j\left(U_{1}-C A T\right)$. Nas condições acima temos: 
(i) $J$ hom-bijetor $\Rightarrow \forall(c, g) \in(J \rightarrow a)_{0}\left(\pi_{0}((c, g) \rightarrow \phi(a . I)(H, \mu)) \cong\left\{M \in \pi_{0}(c \rightarrow H)\right.\right.$ : $\left.\left.\Gamma_{c}(M)=g\right\}\right)$.

(ii) $J$ hom-bijetor $\wedge\left(J_{0}\right)_{*}\left(C_{0}\right) \subseteq A_{\mathcal{I}} \Rightarrow\left(\mu \in[I, A]\left(J \circ H, \Delta_{I}(a)\right)\right.$ limite $\Rightarrow \Gamma_{c}$ bijeção $)$.

(iii) $J$ hom-bijetor $\wedge\left(J_{0}\right)_{*}\left(C_{0}\right) \subseteq A_{\mathcal{I}} \Rightarrow\left(\mu \in[I, A]\left(J \circ H, \Delta_{I}(a)\right)\right.$ limite $\Rightarrow \phi(a, I)(H, \mu)$ final ).

(iv) $J$ denso $\Rightarrow\left(T \in U_{1}-C A T(I,(J \rightarrow a))\right.$ final $\Rightarrow \psi(a, I)(T)=(H, \mu)(\mu$ limite $\left.)\right)$.

(v) $J$ denso $\wedge J$ hom-bijetor $\wedge\left(J_{0}\right)_{*}\left(C_{0}\right) \subseteq A_{\mathcal{I}} \Rightarrow \phi(a, I)$ I: $\{(H, \mu) \in X(a, I): \mu$ limite \}$\rightleftarrows\{T \in Z(a, I): T$ final $\}: \psi(a, I) \mid$ bijeções inversas.

(vi) $\left(J\right.$ denso $\wedge J$ hom-bijetor $\wedge\left(J_{0}\right)_{*}\left(C_{0}\right) \subseteq A_{\mathcal{I}} \wedge A \mathcal{I}$-ind completa $) \Rightarrow\left\{a \in A_{0}\right.$ : $\exists I \in \mathcal{I} \exists H: I \rightarrow C(a \underset{I}{\stackrel{\lim }{\rightarrow}} J \circ H)\}=\left\{a \in A_{0}: \exists I \in \mathcal{I} \exists T: I \rightarrow(J \rightarrow a)(T\right.$ final $\left.)\right\}$.

Para (i) , contemplar o diagrama:

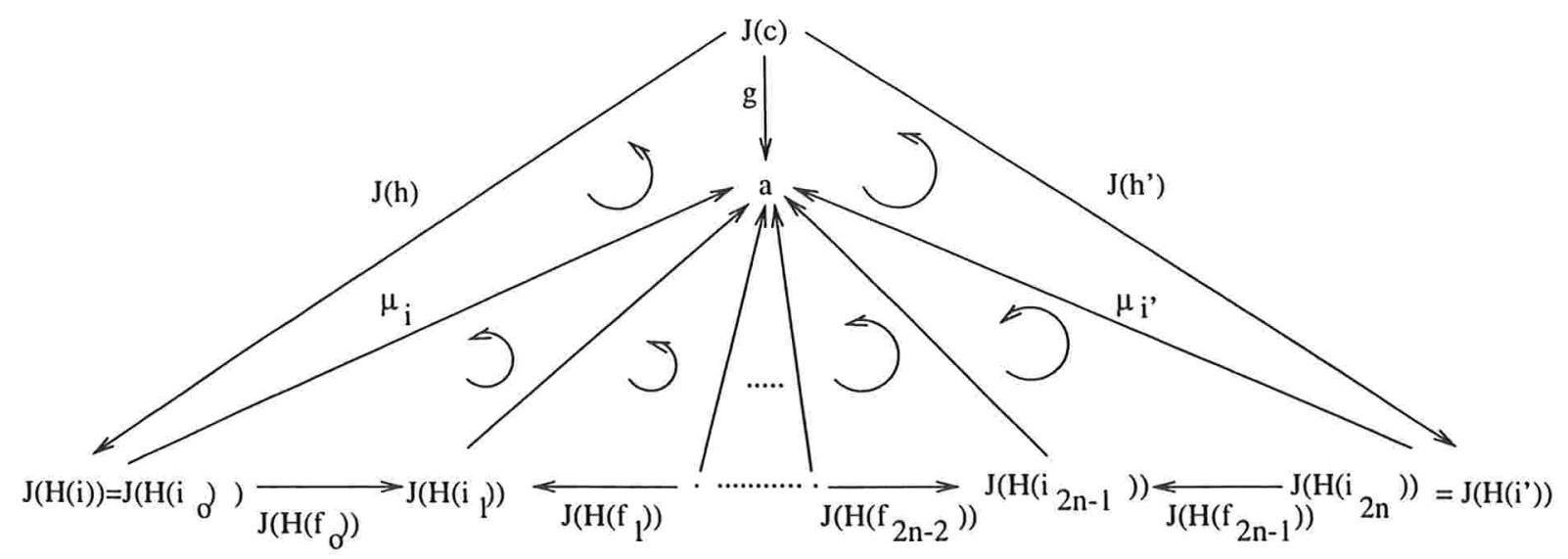




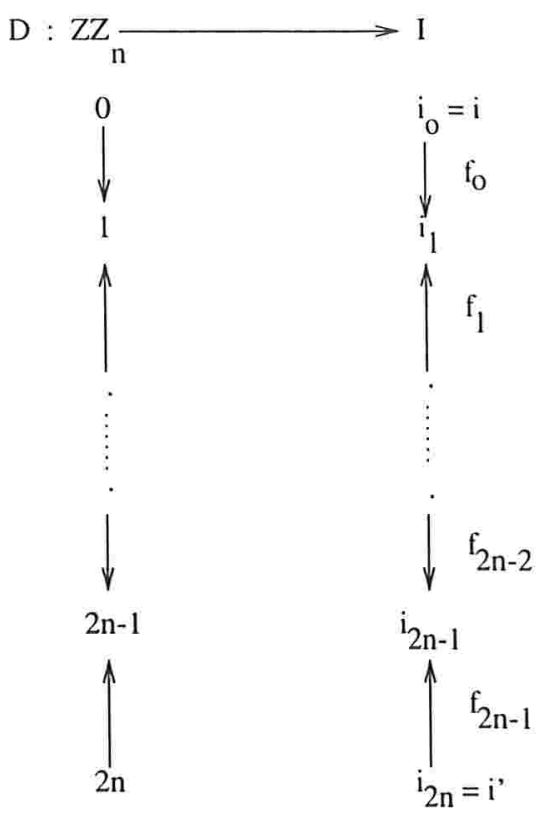

Proposição 2 : Sejam $J \in U_{1}-C A T(C: A) \mathcal{I}$-ind completamento forte de $C$ e $B$ $U_{1}$-categoria $\mathcal{I}$-ind completa onde $\mathcal{I}-\{\emptyset\} \neq \emptyset$.

(i) $A_{0}=\left\{a \in A_{0}: \exists I \in \mathcal{I} \exists H: I \rightarrow C(a \underset{I}{\longrightarrow} \lim J \circ H)\right\}=\left\{a \in A_{0}: \exists I \in \mathcal{I}\right.$ $\exists T: I \rightarrow(J \rightarrow a)(T$ final $)\}$.

(ii) $J$ é denso.

(iii) $\forall F: C \rightarrow B \exists L_{J}(F): A \rightarrow B$ (e são pontuais) .

(iv) $\forall F: C \rightarrow B\left(\eta_{F}: F \stackrel{\cong}{\rightrightarrows} L_{J}(F) \circ J\right)$.

(v) $\forall F: C \rightarrow B\left(L_{J}(F)\right.$ preserva $\mathcal{I}$-limites indutivos ).

(vi) $\forall T: A \rightarrow B\left(T\right.$ preserva $\mathcal{I}$-limites indutivos $\Rightarrow \epsilon_{T}: L_{J}(T \circ J) \cong T$ ).

(vii) $J$ é $\mathcal{I}$-ind completamento fraco de $C$.

(i) Segue da proposição acima e da definição de completamento ( e $\mathcal{I}-\{\emptyset\} \neq \emptyset$ ) .

(ii) Decorre de (i) .

(iii) Decorre de (i) utilizando a hipótese $B \mathcal{I}$-ind completa.

(iv) Por (iii) e porque $J$ é hom-bijetor.

(v) Pois $L_{J}(F)$ é $\tilde{J}$-adjunto à esquerda de $\tilde{F}$ e $\left(J_{0}\right)_{*}\left(C_{0}\right) \subseteq A_{\mathcal{I}}$.

(vi) Como $\left(L_{J}[B, J], \eta, \epsilon\right):[C, B] \rightarrow[A, B]$ é adjunção temos $\epsilon_{T} J \circ \eta_{T \circ J}=1_{T \circ J}$; então por (iv) $\epsilon_{T} J=\left(\eta_{T \circ J}\right)^{-1}$. Como $\forall a \in A_{0} \exists I \in \mathcal{I} \exists H: I \rightarrow C \exists \gamma(\gamma$ é cone indutivo limite $\wedge$ $\operatorname{bas\epsilon }(\gamma)=J \circ H \wedge \operatorname{vértic\epsilon }(\gamma)=a)$ e $\epsilon$ é transformação natural, o diagrama abaixo comuta: 
A

$\mathrm{B}$

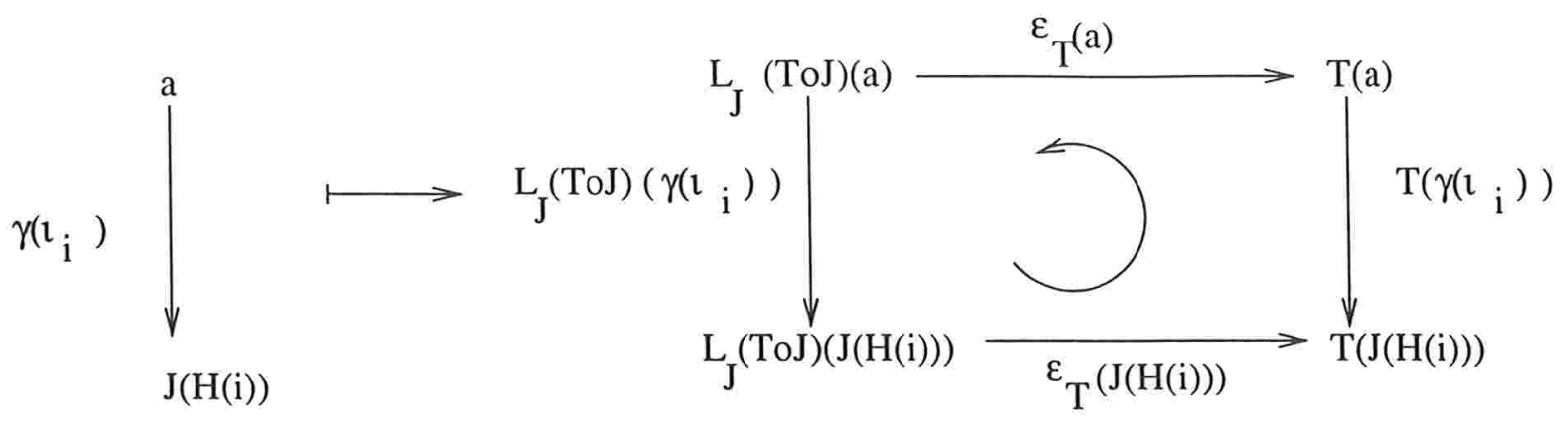

Como por hipótese e por (v) $T$ e $L_{J}(T \circ J)$ preservam $\mathcal{I}$-limites indutivos e $\forall i \in I_{0}$ ( $\left.\epsilon_{T}(J \circ H(i))=\left(\eta_{T \circ J \circ H(i)}\right)^{-1}\right)$ temos que $\epsilon_{T}(a)$ é isomorfismo natural .

(vii) Por (iii),(iv),(v),(vi) .

\section{Observação 4 :}

(i) Se $C$ admite $\mathcal{I}$-ind completamento forte então todo $\mathcal{I}$-ind completamento fraco de $C$ é forte; em particular, completamentos fortes são essencialmente únicos.

(ii) $J: C \rightarrow A \mathcal{I}$-ind completamento forte $\Rightarrow \tilde{J}: A \rightarrow\left[C^{\star}, S E T\right]$ hom-bijetor (e portanto reflete limites projetivos/indutivos) $\wedge \tilde{J}$ preserva $(C A T)_{0}$-limites projetivos e $\mathcal{I}$ - limites indutivos $\Rightarrow I S O(S E T, \mathcal{I}) \cap\left\{P \in(C A T)_{0}: A\right.$ é $P$-proj completa $\} \subseteq I S O(A, \mathcal{I})$.

Observação 5 : Utilizando os resultados acima encontramos candidatos naturais a $\mathcal{I}$-ind completamentos fortes $(\mathcal{I}-\{\emptyset\} \neq \emptyset)$ :

Seja $C^{\prime} U_{1}$-categoria praticamente localmente em $U_{0}$ que admite $\mathcal{I}$-ind completamento forte $J: C \rightarrow A$. Em particular, temos que $A$ é $U_{1}$-categoria praticamente localmente em $U_{0}: J$ é hom-bijetor e denso; logo $\tilde{J} \circ J \cong Y_{C}: C \rightarrow\left[C^{\star}: S E T\right]$ e o mesmo ocorre quando consideramos suas "restrições" a $\left[C^{\star}, U_{0}-S E T\right]$. Sejam $K_{C}(\mathcal{I})$ a subcategoria plena (e fechada por isomorfismos) de $\left[C^{\star}, U_{0}-S E T\right] \operatorname{com} o b j\left(K_{C}(\mathcal{I})\right)=\left\{F \in \operatorname{obj}\left(\left[C^{\star}, U_{0}-S E T\right]\right)\right.$ : $\left.\exists a \in A_{0}(F \cong \tilde{J}(a))\right\}$ e $i_{C}(\mathcal{I}): K_{C}(\mathcal{I}) \hookrightarrow\left[C^{\star}\right.$, $\left.U_{0}-S E T\right]$ então, lembrando que $\tilde{J}$ preserva $\mathcal{I}$-limites indutivos, temos:

(i) $\tilde{J}\left\lceil: A \cong K_{C}(\mathcal{I})\right.$.

(ii) $K_{C}(\mathcal{I})$ é $U_{1}$-categoria $\mathcal{I}$-ind completa e $i_{C}(\mathcal{I})$ preserva $\mathcal{I}$-limites indutivos.

(iii) $K_{C}(\mathcal{I})=\left\{F \in \operatorname{obj}\left(\left[C^{\star}, U_{0}-S E T\right]\right): \exists I \in \mathcal{I} \exists H: I \rightarrow C\left(F \cong \underset{I}{\lim } Y_{C} \circ H\right)\right\}$.

(iv) $Y_{C}(\mathcal{I})=Y_{C} \cong \tilde{J}_{\mid} \circ J: C \rightarrow K_{C}(\mathcal{I})$ é $\mathcal{I}$ - ind completamento forte de $C$.

Temos esses candidatos em mente, mas podemos facilitar nosso trabalho pois:

Proposição 3 : Sejam $\mathcal{I} \subseteq$ obj $\left(U_{0}-C A T\right)$ e $J \in U_{1}-C A T(C, A)$ tais que $\mathcal{I}-\{\emptyset\} \neq \emptyset$ : $A \mathcal{I}$-ind completa . $J$ denso : $J$ hom-bijetor e $\left(J_{0}\right)_{*}\left(C_{0}\right) \subseteq A_{\mathcal{I}}$. Seja $i: \bar{A} \hookrightarrow A$ a inclusão 
da subcategoria plena de $A$ tal que $\bar{A}_{0}=\left\{a \in A_{0}: \exists I \in \mathcal{I} \exists H: I \rightarrow C(a \cong \underset{I}{\lim } J \circ H)\right\}$ Temos :

(i) $\bar{A}_{0}=\left\{a \in A_{0}: \exists I \in \mathcal{I} \exists T: I \rightarrow(J \rightarrow a)\right.$ ( $T$ é final ) $\}$.

(ii) $\left(J_{0}\right)_{*}\left(C_{0}\right) \subseteq \bar{A}_{0}(\operatorname{seja}(C \stackrel{J}{\rightarrow} A)=(C \stackrel{\bar{J}}{\rightarrow} \bar{A} \stackrel{i}{\hookrightarrow} A))$.

(iii) $\bar{J}$ e $i$ são hom-bijetores e densos.

Suponhamos que $i: \bar{A} \hookrightarrow A$ crie $\mathcal{I}$-limites indutivos. Então:

(iv) $\bar{A}$ é $\mathcal{I}$-ind completa.

(v) $\bar{A}_{0}=\left\{\bar{a} \in \bar{A}_{0}: \exists I \in \mathcal{I} \exists H: I \rightarrow C(\bar{a} \cong \underset{I}{\lim } \bar{J} \circ H)\right\}$.

(vi) $\bar{J}_{0}\left(C_{0}\right) \subseteq \bar{A}_{\mathcal{I}}$.

(vii) $\bar{J}: C \rightarrow \bar{A}$ é $\mathcal{I}$-ind completamento forte de $C$.

Observação 6 : Para $C U_{1}$-categoria praticamente localmente em $U_{0}$ existe $J: C \rightarrow A$ como na proposição acima :

Tomando $J: C \rightarrow A$ como a "restrição" do funtor de Yoneda a $\left[C^{\star}, U_{0}-S E T\right]$ temos:

. A é $U_{1}$-categoria $U_{0}$-ind completa;

. $J$ é hom-bijetor;

. $J$ é denso e $\left(J_{0}\right)_{*}\left(C_{0}\right) \subseteq A_{\mathcal{I}}\left(\right.$ pois $\left.\tilde{J} \cong 1_{\left[C^{\star}, U_{0}-S E T\right]}\right)$;

$. \operatorname{obj}(\bar{A})=\operatorname{obj}\left(K_{C}(\mathcal{I})=\left\{a \in A_{0}: \exists I \in \mathcal{I} \exists H: I \rightarrow C(\underset{I}{\longrightarrow} J \circ H)\right\}=\left\{a \in A_{0}:\right.\right.$ $\exists I \in \mathcal{I} \exists T: I \rightarrow(J \rightarrow a)(T$ final $)\}$.

Definição 4: $\mathcal{I} \subseteq o b j\left(U_{0}-C A T\right)$ é diagonalmente indutiva $\Longleftrightarrow$ a subcategoria plena de $U_{0}-C A T$ com objetos $\left.\mathcal{I}^{{ }^{+}}\right|_{U_{0}}=\left\{X \in U_{0}-C A T: \exists I \in \mathcal{I} \exists T: I \rightarrow X(T\right.$ final $\left.)\right\}$ é fechada em $U_{0}-C A T$ por $\mathcal{I}$-limites indutivos.

Exemplos :

(i) $\mathcal{I}=\emptyset=\left.\mathcal{I}^{\mathfrak{}}\right|_{U_{0}}$.

(ii) $\mathcal{I}=\{\emptyset\}=\mathcal{I} \preceq \rightarrow \mid$

(iii) $\mathcal{I}=\sum_{\rightarrow}(S, \mathcal{X}) \cap o b j\left(U_{0}-C A T\right)=\mathcal{I} \preceq \rightarrow{ }_{U_{0}}$ onde $\mathcal{X} \subseteq$ obj(CAT), $S$ é categoria $U_{0}$-ind

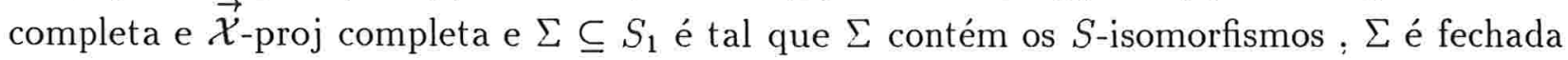
por composição à esquerda e à direita por $S$-isomorfismos e composições à direita de flechas em $\Sigma$ por $U_{0}$-limites indutivos de flechas em $\Sigma$ estão em $\Sigma$ (exemplos : os isomorfismos, as inversíveis à direita, os epimorfismos) .

(iv) $\mathcal{I}=\left\{I \in \operatorname{obj}\left(U_{0}-C A T\right): I^{\star} \in \sum_{\leftarrow}(S, \mathcal{X}) \cap o b j\left(U_{0}-C A T\right)\right\}=\left.\mathcal{I} \preceq{ }^{\rightarrow}\right|_{U_{0}}$ onde $\mathcal{X} \subseteq$ obj $(C A T), S$ é categoria $U_{0}$-proj completa e $\mathcal{X}$-ind completa e $\Sigma \subseteq S_{1}$ é tal que $\Sigma$ contém os $S$-isomorfismos . $\Sigma$ é fechada por composição à esquerda e à direita por $S$ isomorfismos e composições à esquerda de flechas em $\Sigma$ por $U_{0}$-limites projetivos de flechas em $\Sigma$ estão em $\Sigma$ (exemplos : os isomorfismos, as inversíveis à esquerda, os monomorfismos) 
(v) $\mathcal{I}=\left\{I \in o b j\left(U_{0}-C A T\right): I\right.$ é categoria associada a ordem $\kappa$-dirigida $\}:\left.\mathcal{I}^{\preceq}\right|_{U_{0}}=$ $\left\{X \in \operatorname{obj}\left(U_{0}-C A T\right): X\right.$ é categoria $\kappa$-filtrada $\}$ onde $\omega \leq \kappa=r \epsilon g(\kappa)<\# U_{0}$.

(vi) $\mathcal{I}=\left\{I \in \operatorname{obj}\left(U_{0}-C A T\right): I\right.$ é categoria associada a pré-ordem $\kappa$-dirigida $\}:\left.\mathcal{I}^{\prec}\right|_{U_{0}}$ $=\left\{X \in \operatorname{obj}\left(U_{0}-C A T\right): X\right.$ é categoria $\kappa$ - filtrada $\}$ onde $\omega \leq \kappa=\operatorname{reg}(\kappa)<\# U_{0}$.

(vii) $\mathcal{I}=\left\{I \in \operatorname{obj}\left(U_{0}-C A T\right): \# I<\kappa\right\}:\left.\mathcal{I}^{\preceq \rightarrow}\right|_{U_{0}}=I S O\left(U_{0}-S E T,\{X \in\right.$ $\operatorname{obj}\left(U_{0}-C A T\right): X$ é categoria $\kappa$-filtrada $\left.\}\right) \cap \operatorname{obj}\left(U_{0}-C A T\right)$ onde $\omega \leq \kappa=r \epsilon g(\kappa)<\# U_{0}$ (parágrafo 15.8 em [092]) .

(viii) $\mathcal{I}=\left\{I \in \operatorname{obj}\left(U_{0}-C A T\right): I\right.$ é discreta $\}:\left.\mathcal{I}^{\prec \rightarrow}\right|_{U_{0}}=\left\{X \in U_{0}-C A T: X\right.$ admite diagrama $U_{0}$-pequeno multiplamente terminal $\}$.

(ix) $\mathcal{I}=\{\mathbf{E}\}:\left.\mathcal{I}^{\preceq}\right|_{U_{0}}=\left\{X \in U_{0}-C A T: X\right.$ é ind-degenerada $\}$.

Proposição 4: Sejam $\mathcal{I} \subseteq \operatorname{obj}\left(U_{0}-C A T\right)$ diagonalmente indutiva com $\mathcal{I}-\{\emptyset\} \neq \emptyset$ : $C U_{1^{-}}$categoria praticamente localmente em $U_{0}$ e $J: C \rightarrow A$ como na proposição 3 então $i: \bar{A} \hookrightarrow A$ cria $\mathcal{I}$-limites indutivos, $\log$ o $\bar{J}: C \rightarrow \bar{A}$ é $\mathcal{I}$-ind completamento forte de $C$.

Sejam $Z \in \mathcal{I}: \Gamma: Z \rightarrow \bar{A}$ e

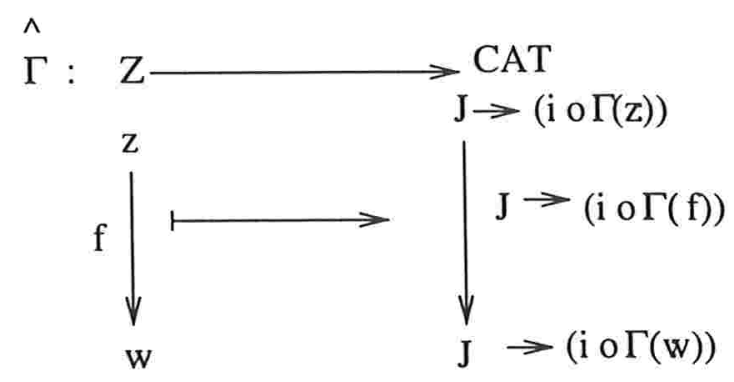

. $C$ praticamente localmente em $U_{0} \Rightarrow \forall z \in Z_{0} \hat{\Gamma}(z)$ é $U_{1}$-categoria praticamente localmente em $U_{0}$.

. $\forall z \in Z_{0} \exists I_{z} \in \mathcal{I} \exists T_{z}: I_{z} \rightarrow \hat{\Gamma}(z)$ ( $T_{z}$ final ) ( fixemos uma escolha !) .

- $\forall w \in Z_{0}$ seja $\Psi(w)$ a subcategoria plena de $\hat{\Gamma}(w) \operatorname{com} \operatorname{obj}(\Psi(w))=\left\{(c, h): c \in C_{0} \wedge\right.$ $\left.h \in A(J(c), i(\Gamma(w))) \wedge \exists z \in Z_{0} \exists i_{z} \in\left(I_{z}\right)_{0} \exists f \in Z(z, w)\left((c, h)=\Gamma^{\prime}(f)\left(T_{z}\left(i_{z}\right)\right)\right)\right\}$.

. $\forall w \in Z_{0}\left(\#\left(\Psi(w)_{0}\right)<\# U_{0}\right)$.

- $\forall w \in Z_{0}\left(\Psi(w)\right.$ é $U_{1}$-categoria praticamente em $\left.U_{0}\right)$.

. $\forall z \in Z_{0}$ o funtor final $T_{z}$ se fatora como a composição de funtores finais $I_{z} \stackrel{T_{2}}{\rightarrow} \Psi(z) \hookrightarrow$ $\hat{\Gamma}(z)$

. $\forall f: z \rightarrow w\left(\left(\hat{\Gamma}(f)_{0}\right)_{*}\left(\Psi(z)_{0}\right) \subseteq \Psi(w)_{0}\right)$ ( $\Psi$ é subfuntor de $\left.\hat{\Gamma}\right)$.

. $\exists \Xi: Z \rightarrow U_{0}-C A T\left(\Psi \cong Z \rightrightarrows U_{0}-C A T \hookrightarrow C A T \wedge \forall z \in Z_{0}\right.$ ( o funtor final $T_{z}$ se fatora como a composição de funtores finais $I_{z} \rightarrow \Xi(z) \stackrel{j_{2}}{\rightarrow} \hat{\Gamma}(z)$ ) : em particular, $\forall z \in Z_{0}$ ( $\left.\Xi(z) \in \mathcal{I}^{\preceq \rightarrow} \cap\left(\operatorname{obj}\left(U_{0}-C A T\right)\right)\right)$.

Temos $\underset{Z}{\lim } i(\Gamma(z)) \in \operatorname{obj}(\bar{A})$ pois: 


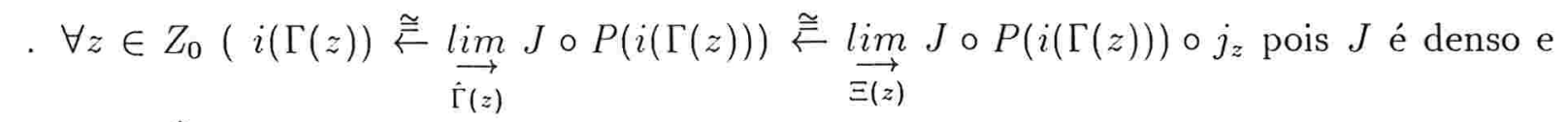
$j_{z}: \Xi(z) \multimap \hat{\Gamma}(z)$ é final.

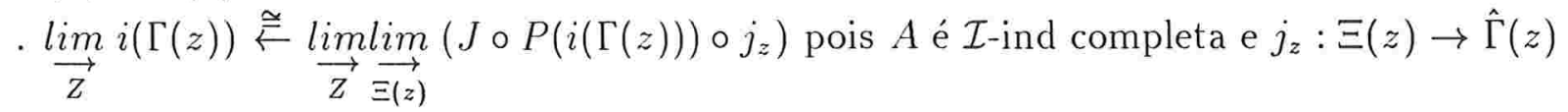
e $P(i(\Gamma(z))):(J \rightarrow i(\Gamma(z))) \rightarrow C$ são naturais em $z \in Z_{0}$.

- $\underset{Z}{\lim } \underset{\Xi(z)}{\longrightarrow}\left(J \circ P(i(\Gamma(z))) \circ j_{z}\right) \stackrel{\cong}{\rightarrow} \underset{X}{\lim } J \circ L$ onde $X=\underset{Z}{\lim } \Xi(z)$ e $L: X \rightarrow C$ pela proposição 1 do ítem (1.3.1) .

. $\underset{X}{\lim } J \circ L \underset{I}{\stackrel{\lim }{\rightleftarrows}} J \circ L \circ T$ para algum $I \in \mathcal{I}$ e algum $T: I \rightarrow X$ final ; pois $\mathcal{I}$ é diagonalmente indutiva.

. $\underset{I}{\lim } J \circ L \circ T \in \operatorname{obj}(\bar{A})$ pela definição de $\bar{A}$.

Definição 5 : Sejam $S U_{1}$-categoria praticamente localmente em $U_{0}$ e $M: S \rightarrow U_{0}-S E T$ funtor :

(i) $K(M)$ é a categoria com $o b j(K(M))=|M|=\coprod_{s \in S_{0}} M(s): K(M)\left((x, s),\left(x^{\prime}, s^{\prime}\right)\right)=$ $\{(x, s)\} \times\left\{f \in S\left(s, s^{\prime}\right): M(f)(x)=x^{\prime}\right\} \times\left\{\left(x^{\prime}, s^{\prime}\right)\right\}$ com funções composição e identidade "derivadas das de $S$ ".

(ii) $\# M=\#|M|=\left(\sum_{s \in S_{0}} \# M(s)\right)$.

Observação 7 :

(i)

. $S U_{1}$-categoria em $U_{0} \Rightarrow \forall M: S \rightarrow U_{0}-S E T\left(K(M) U_{1}\right.$-categoria em $\left.U_{0}\right)$.

. $S U_{1}$-categoria praticamente em $U_{0} \Rightarrow \forall M: S \rightarrow U_{0}-S E T$ ( $K(M) U_{1}$-categoria praticamente em $U_{0}$ ).

. $S U_{1}$-categoria essencialmente em $U_{0} \Rightarrow \forall M: S \rightarrow U_{0}-S E T\left(K(M) U_{1}\right.$-categoria essencialmente em $\left.U_{0}\right)$.

(ii) $\left(\Delta_{1}(1) \rightarrow M\right) \cong K(M) \cong\left(Y_{S^{*}} \rightarrow M\right)^{\star}$.

Proposição 5 : Sejam $\mathcal{I} \subseteq$ obj $\left(U_{0}-C A T\right), \mathcal{P} \subseteq I S O\left(U_{0}-S E T, \mathcal{I}\right) \cap o b j\left(U_{0}-C A T\right), C$ $U_{1}$ - categoria praticamente localmente em $U_{0}$ e $F: C^{\star} \rightarrow U_{0}-S E T$ funtor. Consideramos:

(i)

. $\exists I \in \mathcal{I}\left(K(F)^{\star}=I\right)$.

. $\exists I \in \mathcal{I}\left(K(F)^{\star} \cong I\right)$.

. $\exists I \in \mathcal{I}\left(K(F)^{\star} \simeq I\right)$.

(ii) $\exists I \in \mathcal{I} \exists T: I \rightarrow\left(Y_{C} \rightarrow F\right)$ ( $T$ é final ).

(iii) $\exists I \in \mathcal{I} \exists H: I \rightarrow C\left(F \cong \underset{I}{\lim } Y_{C} \circ H\right)$.

(iv) $F \in\left[\left\{C(-, c): c \in C_{0}\right\}\right] \stackrel{\mathcal{I}-\lim }{\rightarrow}$. 
(v) $\exists L_{Y_{C^{\star}}}(F):\left[C . U_{0}-S E T\right] \rightarrow U_{0}-S E T$, é pontual e preserva $\mathcal{P}$-limites projetivos.

(vi") $F$ preserva $\mathcal{P}$-limites projetivos.

(vi $\left.{ }^{\prime}\right) F$ funde diagramas pequenos multiplamente limites projetivos de funtores $\mathcal{P}$-indexados , i.e. $\forall P \in \mathcal{P} \forall P \stackrel{D}{\rightarrow} C^{*}\left(\exists I \cong \mathcal{D}\left(I_{0}\right) \in \operatorname{obj}\left(U_{0}-C A T\right) \exists L: I \rightarrow C^{*} \exists \eta: \bigcup_{i \in I_{0}} C^{*}\left({ }_{-}, c_{i}\right) \stackrel{\cong}{\stackrel{\cong}{\leftrightarrows}} \underset{P}{\lim }\right.$ $C^{\star}(-, D(p)) \Rightarrow \underset{i \in I_{0}}{\coprod_{0}} F \circ L(i) \underset{\stackrel{c a p}{\rightarrow} \lim }{\stackrel{i m}{\leftrightarrows}} F \circ D(p)$ é isomorfismo $)$.

(vi) $\exists L_{Y_{C^{*}}}(F):\left[C, U_{0}-S E T\right] \rightarrow U_{0}-S E T$, é pontual e preserva limites projetivos de diagramas $\Gamma$ tais que $\left(P \stackrel{\Gamma}{\rightarrow}\left[C, U_{0}-S E T\right]\right)=\left(P \stackrel{D}{\rightarrow} C^{*} \stackrel{Y_{C C^{*}}}{\rightarrow}\left[C, U_{0}-S E T\right]\right)$ onde $P \in \mathcal{P}$.

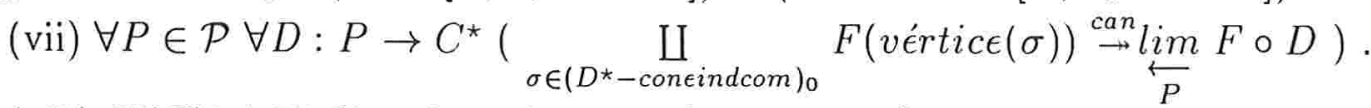

(viii) $K(F)^{\star}$ é $\mathcal{P}^{\star}$-filtrada onde $\mathcal{P}^{\star}=\left\{P^{\star}: P \in \mathcal{P}\right\}$.

Temos:

(1) (ii) $\Longleftrightarrow$ (iii) $\Rightarrow$ (iv) $\Rightarrow$ (v) $\Rightarrow$ (vi) $\Rightarrow$ (vii $\Rightarrow$ (viii) .

(2) $C$ essencialmente em $U_{0} \Rightarrow$ ( (i) $\Rightarrow$ (ii) ) .

(3) $\mathcal{I}$ diagonalmente indutiva $\Rightarrow$ ( (iii) $\Leftarrow$ (iv) ) .

(4) $\exists \omega<\kappa=r \epsilon g(\kappa)<\# U_{0}\left(\mathcal{I}=\left\{I \in \operatorname{obj}\left(U_{0}-C A T\right): I\right.\right.$ é categoria $\kappa$-filtrada $\} \wedge$ $\left.\left.\mathcal{P}=\left\{P \in \operatorname{obj}\left(U_{0}-C A T\right): \# P<\kappa\right\}\right)\right) \wedge\left(\right.$ respectivamente: $C$ em $U_{0} ; C$ praticamente em $U_{0}: C$ essencialmente em $\left.U_{0}\right) \Rightarrow$ (respectivamente: (viii) $\Rightarrow($ i) ) .

(5) $\left(\mathrm{vi}^{\prime \prime}\right) \Leftarrow\left(\right.$ vi) e se $C$ está essencialmente em $U_{0}$ e $C$ é $\mathcal{P}^{\star}$-ind completa então (vi") $\Rightarrow$ (vi)

(6) $\left(\mathrm{vi}^{\prime}\right) \Leftarrow$ (vi) e se $C$ está essencialmente em $U_{0}$ e $C$ admite diagramas pequenos multiplamente limites indutivos de funtores $\mathcal{P}^{\star}$-indexados então $\left(\mathrm{vi}^{\prime}\right) \Rightarrow(\mathrm{vi})$.

(1) Vamos demonstrar apenas (iv) $\Rightarrow(v)$; (vi) $\Rightarrow$ (vii) ; (vii) $\Rightarrow$ (viii) pois os outros ítens ou são triviais ou já foram considerados acima.

(iv) $\Rightarrow$ (v)

Seja $\left(X_{\alpha}\right)_{a \in O R D^{+}}$a seqüência crescente de classes de objetos de subcategorias plenas e repletas de $\left[C^{\star}, U_{0}-S E T\right]$ tal que:

. $\alpha=0 \Rightarrow X_{\alpha}=\left\{F \in\right.$ obj $\left.\left(\left[C^{\star}, U_{0}-S E T\right]\right): \exists c \in C_{0}(F \cong C(-, c))\right\}$.

. $0 \neq \alpha=\mathrm{V}\left[0, \alpha\left[\Rightarrow X_{\alpha}=\cup_{\beta<\alpha} X_{\beta}\right.\right.$.

. $\exists \beta(\alpha=\beta+1) \Rightarrow X_{\alpha}=X_{\beta} \cup X_{\beta}(\mathcal{I})$ onde $X_{\beta}(\mathcal{I})=\left\{F \in \operatorname{obj}\left(\left[C^{\star}, U_{0}-S E T\right]\right): \exists I \in \mathcal{I}\right.$ $\left.\exists\left(I \stackrel{\Psi}{\rightarrow}\left[C^{\star}, U_{0}-S E T\right]\right)=\left(I \stackrel{\Psi_{\beta}}{\rightarrow} \operatorname{plena}\left(X_{\beta}\right) \hookrightarrow \operatorname{obj}\left(\left[C^{\star}, U_{0}-S E T\right]\right)\right)(F \underset{I}{\stackrel{\lim }{\rightarrow}} \Psi(i))\right\}$.

. $\left[\left\{C(-, c): c \in C_{0}\right\}\right] \stackrel{\mathcal{I - l i m}}{\rightarrow}=X_{\# U_{0}}$.

Pois $\mathcal{I} \subseteq \operatorname{obj}\left(U_{0}-C A T\right)$ e $\# U_{0}$ é cardinal infinito (ordinal limite!) e regular.

. $\left.\alpha=0 \Rightarrow \exists c \in C_{0}\left(L_{Y_{C^{*}}}(F) \cong L_{Y_{C^{*}}}\left(C^{\star}(c,-)\right) \cong\left[C^{\star}, U_{0}-S E T\right]\left(Y_{C^{\star}}(c)\right)_{--}\right)\right)$, é pontual e preserva $U_{0}$-limites projetivos.

$.0 \neq \alpha=\mathrm{V}[0, \alpha[\mathrm{ok}$. 
. $\exists \beta(\alpha=\beta+1)$ se $F \in X_{\beta}(\mathcal{I})(F \cong \underset{I}{\lim } \Psi(i))$. como $\left[C^{\star} . U_{0}-S E T\right]$ é $\mathcal{I}$-ind completa : $L_{Y_{C^{\star}}}$ é adjunto à esquerda parcial máximo de $\left[Y_{C^{\star}}, U_{0}-S E T\right]$ e pela hipótese de indução , temos que $\exists L_{Y_{C^{*}}}(F) \cong \underset{I}{\lim } L_{Y_{C^{*}}}(\Psi(i))$ e é pontual : pela relação entre $\mathcal{P}$ e $\mathcal{I}$ e pela hipótese de indução temos que $L_{Y_{C^{\star}}}(F)$ preserva $\mathcal{P}$-limites projetivos.

(vi) $\Rightarrow$ (vii)

. $\lim _{P}\left(L_{Y_{C^{*}}}(F) \circ Y_{C^{\star}} \circ D(p)\right) \cong \lim _{P} F \circ D(p)$.

Pois $Y_{C^{*}}$ é hom-bijetor e $L_{Y_{C^{*}}}(F)$ é pontual.

. $L_{Y_{C^{\star}}}(F)\left(\underset{P}{\lim } Y_{C^{\star}} \circ D(p)\right) \stackrel{\text { cañ }}{\leftarrow}\left(\underset{\sigma \in\left(D^{\star}-\text { contindcom }\right)_{0}}{\coprod_{P}} F(\right.$ vértict $\left.(\sigma))\right) / \equiv$.

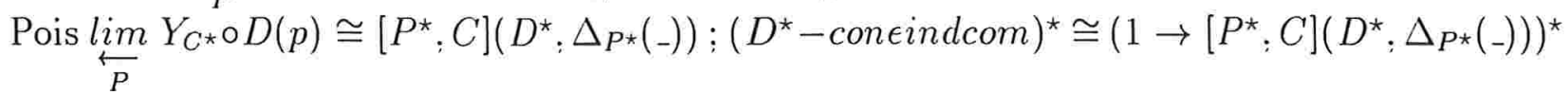
$\cong\left(Y_{C^{\star}} \rightarrow\left[P^{\star}: C\right]\left(D^{\star}: \Delta_{P^{\star}}(-)\right)\right) ; \forall M: C \rightarrow U_{0}-\operatorname{SET}\left(L_{Y_{C^{\star}}}(F)(M) \cong \stackrel{\lim }{\longrightarrow}\left(\left(Y_{C^{\star}} \rightarrow M\right) \stackrel{P(M)}{\rightarrow}\right.\right.$

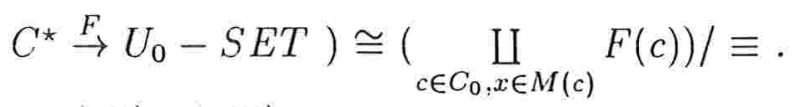

(vii) $\Rightarrow$ (viii)

. $\forall P \in \mathcal{P} \forall D: P \rightarrow C^{\star} \forall \Xi: P^{\star} \rightarrow\left(Y_{C} \rightarrow F\right)\left(P(F) \circ \Xi=D^{\star} \Rightarrow U_{1}-C A T\left(P^{\star}, K(F)^{\star}\right)\right.$ $\cong U_{1}-C A T\left(P^{\star},\left(Y_{C} \rightarrow F\right)\right) \cong\left[P^{\star},\left[C^{\star}: U_{0}-S E T\right]\right]\left(\left(Y_{C} \circ D^{\star}: \Delta_{P^{\star}}(F)\right) \cong\right.$ $\lim _{P}\left[C^{\star}, U_{0}-S E T\right]\left(Y_{C} \circ D^{\star}(p), F\right) \cong \underset{P}{\lim } F \circ D(p)$.

Logo afirmar que todas as flechas canônicas são sobrejetoras é afirmar que $K(F)^{\star}$ é $\mathcal{P}^{*}$-filtrada.

(2) Observação 7 .

(3) Pois assim $Y_{C}(\mathcal{I}): C \rightarrow K_{C}(\mathcal{I})$ é $\mathcal{I}$-ind completamento forte de $C$ por $\mathcal{I}$-limites indutivos.

(4) Pela observação 7 e porque $\mathcal{P}^{\star}=\mathcal{P}$.

$\left(\mathrm{vi}^{\prime \prime}\right) \Leftarrow(\mathrm{vi})$

Pois $F \stackrel{\eta_{F} \cong}{\rightarrow} L_{Y_{C^{\star}}}(F) \circ Y_{C^{\star}}$ e $Y_{C^{\star}}: C^{\star} \rightarrow\left[C, U_{0}-S E T\right]$ preserva todos os $U_{0^{-}}$-limites projetivos em $C^{\star}$.

$\left(\mathrm{vi}^{\prime \prime}\right) \Rightarrow(\mathrm{vi})$

Pois $\left(Y_{C^{\star}} \rightarrow F\right)$ está essencialmente em $U_{0}$ logo $\exists L_{Y_{C^{\star}}}(F)$ e é pontual ; assim podemos argumentar como acima.

(6)

$\left(\mathrm{vi}^{\prime}\right) \Leftarrow(\mathrm{vi})$

Pois $F \stackrel{\eta_{F} \cong}{\longrightarrow} L_{Y_{C^{\star}}}(F) \circ Y_{C^{\star}}$ e $L_{Y_{C^{*}}}$ preserva todos os $U_{0^{-}}$limites indutivos preservados por $\tilde{Y_{C^{\star}}} \cong 1_{\left[C, U_{0}-S E T\right]}:\left[C, U_{0}-S E T\right] \rightarrow\left[C, U_{0}-S E T\right]$.

$\left(\mathrm{vi}^{\prime}\right) \Rightarrow(\mathrm{vi})$ 
Pois $\left(Y_{C^{*}} \rightarrow F\right)$ está essencialmente em $U_{0}$ logo $\exists L_{Y_{C^{*}}}(F)$ e é pontual , assim podemos argumentar como acima.

Corolário : $C U_{1}$-categoria essencialmente em $U_{0} \Rightarrow \epsilon v_{C} l: C \rightarrow\left[\left[C, U_{0}-S E T\right], U_{0}-\right.$

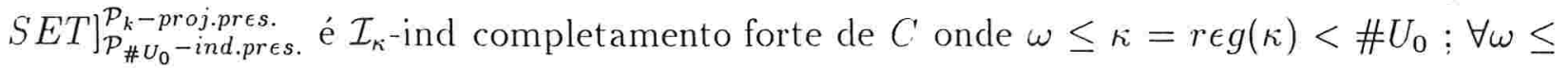
$\lambda=\operatorname{reg}(\lambda) \leq \# U_{0}\left(\mathcal{P}_{\lambda}=\left\{P \in \operatorname{obj}\left(U_{0}-C A T\right): \# P<\lambda\right\} \wedge \mathcal{I}_{\lambda}=\left\{I \in \operatorname{obj}\left(U_{0}-C A T\right):\right.\right.$ $\forall P \in \mathcal{P}_{\lambda}(I$ é $P$-filtrada ) $\}$ ). 


\section{Capítulo 2}

\section{Categorias acessíveis}

A noção central deste trabalho é a de categoria acessível, ou melhor, de $\mathcal{A C} \mathcal{E}$; a 2categoria com esses objetos. Esta é uma definição estratificada: temos dois aspectos no que refere a ela: em cada estágio as propriedades dos completamentos indutivos e num segundo momento a possibilidade de "passeios curtos" pelos estágios serem efetivados no futuro (uniformização ).

O germe da noção de acessibilidade aparece em [098] : mas nossa acepção do termo "categoria acessível" se origina de [166] . Deste permaneceremos próximos neste capítulo.

Fixamos três universos $U_{0} \in U_{1} \in U_{2} \in V$ com cardinais (fortemente inacessíveis) respectivamente $\theta_{0}<\theta_{1}<\theta_{2}<\infty$.

Definição 1 : Sejam $\omega \leq \kappa<\theta_{0}$ e $\mathcal{I}_{\kappa}=\left\{I \in \operatorname{obj}\left(U_{0}-C A T\right): I\right.$ é categoria $\kappa$-filtrada $\}$

(i) $A$ é $U_{1}$-categoria $\kappa$-acessivel $\Longleftrightarrow \exists C U_{1}$-categoria essencialmente em $U_{0} \exists J: C \rightarrow A$ $\mathcal{I}_{\kappa}$-ind completamento forte de $C$.

(ii) $\kappa-\mathcal{A C E}$ é a 2 -categoria com 0 -flechas as categorias (em $\left.U_{1}\right) \kappa$-acessíveis, 1 -flechas os funtores entre duas 0 -flechas que preservam limites indutivos (em $U_{0}$ ) $\kappa$-filtrados, 2-flechas as transformações naturais entre duas 1-flechas e que é sub-2-categoria localmente plena da 2-categoria $U_{1}-\mathcal{C} \mathcal{A} \mathcal{T}$ das $U_{1^{-}}$categorias, funtores e transformações naturais.

(iii) $\kappa-A C E$ é a categoria ordinária associada a $\kappa-\mathcal{A C E}$.

Observação 1 :

(i) A metaclasse $\left(\in U_{2}\right.$ !) das categorias $\kappa$-acessíveis não se altera quando em (i) acima consideramos $C \in \operatorname{obj}\left(U_{0}-C A T\right)$ ou $C$ categoria praticamente em $U_{0}$ e $J: C \hookrightarrow A$.

(ii) Como $\mathcal{I}_{\kappa}=\mathcal{I}_{r \in g(\kappa)}$ sempre tomaremos $\kappa$ regular.

(iii) $\kappa-\mathcal{A C E}=r \in g(\kappa)-\mathcal{A C E} \neq \emptyset$.

(iv) $A \kappa$-acessível $\Rightarrow A$ Karoubiana ( $\Longleftrightarrow$ todo indempotente de $A$ cinde ). 
(v) Utilizaremos as simplificações : $A_{\mathcal{I}_{\kappa}} \sim A_{\kappa}$ ( objeto $\mathcal{I}_{\kappa^{-}}$presentável $\sim$ objeto $\kappa$ presentável ): $Y_{C}\left(\mathcal{I}_{\kappa}\right): C \rightarrow K_{C}\left(\mathcal{I}_{\kappa}\right) \nsim Y_{C}(\kappa): C \rightarrow K_{C}(\kappa) \ldots$

Proposição 1 : Seja $C U_{1}$-categoria praticamente em $U_{0}$ E-proj completa e $\mu=\omega+\# C$ então $Y_{C}\left(\mu^{++}\right): C \stackrel{\widetilde{\rightrightarrows}}{\rightarrow} K_{C}\left(\mu^{++}\right)$e portanto $C$ é $\mu^{++}$-acessivel.

Como $C$ é E-proj completa, $Y_{C}: C \rightarrow\left[C^{\star}, U_{0}-S E T\right]$ preserva $U_{0}$-limites projetivos e $\mathbf{E}$ limites projetivos correspondem a E-limites indutivos temos $Y_{C}(\{\mathbf{E}\}): C \stackrel{\widetilde{\rightrightarrows}}{\rightarrow} K_{C}(\{\mathbf{E}\}) \operatorname{logo}$ basta mostrarmos que $K_{C}\left(\mathcal{I}_{\mu^{++}}\right) \subseteq K_{C}(\{\mathbf{E}\})$ (a inclusão reversa sempre ocorre).

. $\forall F \in o b j\left(K_{C}\left(\mathcal{I}_{\mu^{+}+}\right)\right) \forall c \in C_{0}(\# F(c) \leq \mu)$.

Dados $c \in C_{0}$ e $\left\{x_{i}\right\}_{i \in I} \subseteq F(c)$ com \#I $\leq \mu^{+}$consideremos $G$ o grafo discreto com $G_{0}=I$ e o homomorfismo de grafos $\Gamma: G \rightarrow \mathcal{E}\left(K(F)^{\star}\right)$ tal que $\forall i \in I\left(\Gamma(i)=\left(c ; x_{i}\right)\right)$. Como $K(F)^{\star} \cong\left(Y_{C} \rightarrow F\right)$ é $\mu^{++}$-filtrada, existem $c^{\prime} \in C_{0}: z \in F\left(c^{\prime}\right):\left\{f_{i}\right\}_{i \in I} \subseteq C\left(c, c^{\prime}\right)$ tais que $\forall i \in I\left(x_{i}=F\left(f_{i}\right)(z)\right)$. Como $\# C_{1} \leq \mu$, temos $\#\left\{x_{i}\right\}_{i \in I} \leq \mu ; \operatorname{logo} \# F(c) \leq \mu$.

$$
\text { . } \forall F \in \operatorname{obj}\left(K_{C}\left(\mathcal{I}_{\mu^{++}}\right)\right)\left(\#\left(Y_{C} \rightarrow F\right) \leq \mu\right) \text {. }
$$

$\#\left(Y_{C} \rightarrow F\right) \leq \# C_{0} \cdot \sup \left\{\# F(c): c \in C_{0}\right\}+\# C_{1} \cdot\left(\# C_{0} \cdot \sup \left\{\# F(c): c \in C_{0}\right\}\right)^{2} \leq$ $\mu \cdot \mu+\mu \cdot(\mu \cdot \mu)^{2}=\mu$.

$$
\text { . } K_{C}\left(\mathcal{I}_{\mu^{++}}\right) \subseteq K_{C}(\{\mathbf{E}\}) \text {. }
$$

Dado $F \in \operatorname{obj}\left(K_{C}\left(\mathcal{I}_{\mu^{++}}\right)\right)$temos que $\left(Y_{C} \rightarrow F\right)$ é ind-degenerada logo $\exists \mathbf{E} \stackrel{T}{\rightarrow}\left(Y_{C} \rightarrow F\right)$ final e então $F \in K_{C}(\{\mathbf{E}\})$.

Sabemos, por (1.3.3); que as categorias $\kappa$-acessíveis existem. Abaixo reconheceremos alguns exemplos simples.

Exemplo 1 : A categoria 1 é $\kappa$-acessível para todo $\omega \leq \kappa=r \epsilon g(\kappa)<\theta_{0}$ : o funtor $i d_{1}: \mathbf{1} \rightarrow \mathbf{1}$ é $\mathcal{I}_{\kappa}$-ind completamento forte de $\mathbf{1}$.

Exemplo 2: $U_{0}-S E T$ é $\kappa$-acessível para todo $\omega \leq \kappa=r \epsilon g(\kappa)<\theta_{0}$.

. $\left\{x \in U_{0}: \# x=1\right\} \subseteq\left(U_{0}-S E T\right)_{\kappa}$.

Pois $U_{0}-S E T(1, x) \cong x$ naturalmente em $x \in U_{0}$.

. $x \in U_{0}: \# x<\kappa \subseteq\left(U_{0}-S E T\right)_{\kappa}$.

Pois $\forall x \in U_{0}\left(x=\coprod_{y \in x}\{y\}\right)$.

- $\forall x \in U_{0}$ ( $x$ é $\mathcal{I}_{\kappa}$-limite indutivo de conjuntos de cardinais $<\kappa$ (" $\kappa$-pequenos") ) .

$\kappa$ sendo regular e infinito implica que $\left([x]^{<\kappa}, \subseteq\right)$ é conjunto ordenado $\left(\in U_{0}\right) \kappa$-dirigido; portanto sua categoria associada $I(x, \kappa) \in \mathcal{I}_{\kappa}$; como $\cup[x]^{\kappa}=x$ o funtor "inclusão" $F$ : $I(x, \kappa) \longmapsto U_{0}-S E T$ é tal que $\underset{I(x, \kappa)}{\lim } F(i)=x$.

. A subcategoria plena de $U_{0}-S E T$ dos conjuntos $\kappa$-pequenos está essencialmente em $U_{0}$. 
Pois seu esqueleto é (isomorfo a) subcategoria plena de $U_{0}-S E T$ com objetos os cardinais $\kappa$-pequenos e esta é uma $L_{0}$-categoria ( tem cardinal $\left.\leq\left(\kappa+\kappa^{2} \cdot \kappa^{\kappa}\right)=\kappa^{\kappa}<\theta_{0}\right)$.

- $p l \epsilon n a\left(\left\{x \in U_{0}: \# x<\kappa\right\}\right) \hookrightarrow U_{0}-S E T$ é $\mathcal{I}_{\kappa}$-ind completamento forte de categoria essencialmente em $U_{0}$.

Temos ainda que:

. $\left(U_{0}-S E T\right)_{\kappa}=\left\{x \in\left[I_{0}: \# x<\kappa\right\}\left(=\left[U_{0}\right]^{<\kappa}\right)\right.$.

Pois $\left(U_{0}-S E T\right)_{\kappa}=\left[\left\{x \in U_{0}: \# x<\kappa\right\}\right]^{\{\mathrm{E}\}-\lim } \rightarrow=\left\{x \in U_{0}: \# x<\kappa\right\}$.

Exemplo 3 : Dada $S$ categoria $\left(\right.$ em $\left.U_{1}\right)$ praticamente em $U_{0}\left[S, U_{0}-S E T\right]$ é $\kappa$-acessivel para todo $\omega \leq \kappa=r \epsilon g(\kappa)<\theta_{0}$ tal que $\# S<\kappa$.

. Os exemplos anteriores tratam dos casos $\# S \in\{0,1\}$. Para $S \neq \emptyset$ e $\kappa$ como acima temos :

. $\forall s \in S_{0}\left(S(s,-) \in\left[S, U_{0}-S E T\right]_{\kappa}\right)$.

Pois $\forall s \in S_{0}\left(\left[S, U_{0}-S E T\right](S(s,-), M) \cong M(s)\right)$ naturalmente em $M \in \operatorname{obj}\left(\left[S, U_{0}-\right.\right.$ $S E T])$ e $U_{0}$-limites indutivos em $\left[S, U_{0}-S E T\right]$ são pontuais.

. $\forall M: S \rightarrow U_{0}-\operatorname{SET}\left(\# M<\kappa \Rightarrow \#\left(Y_{S} \rightarrow M\right)<\kappa\right)$.

Pois $\#\left(Y_{S} \rightarrow M\right) \leq \# M+\# S_{1} \cdot(\# M)^{2}<\kappa+\kappa \cdot(\kappa)^{2}=\kappa$.

. $\left\{M: S \rightarrow U_{0}-S E T: \# M<\kappa\right\} \subseteq\left[S, U_{0}-S E T\right]_{\kappa}$.

Pois $Y_{S}: S^{*} \rightarrow\left[S, U_{0}-S E T\right]$ é denso

. $\forall M: S \rightarrow U_{0}-S E T\left(\left(S u b(M)^{<\kappa}\right.\right.$; $\left.\left.\leq\right)\right)$ é conjunto ordenado $\kappa$-dirigido.

Escolhendo os representantes canônicos das classes de equivalência de monomorfismos de codomínio $M$ e: para cada $X \subseteq|M|$ : denotando $\langle X\rangle$ o subfuntor gerado por $X$ temos $\forall t \in S_{0}\left(<X>(t)=\left\{z \in M(t): \exists s \in S_{0} \exists f: s \rightarrow t \exists y \in X(s)(z=M(f)(y))\right\} \operatorname{logo}\right.$ $\#<X>\leq \# S_{0} \cdot \# S_{0} \cdot \# S_{1} \cdot \# X<\kappa$.\#X . Como $\kappa$ é regular dada $\left\{M_{p}\right\}_{p \in P} \subseteq \operatorname{Sub}(M)^{<\kappa} \operatorname{com}$ $\# P<\kappa$ temos \# $\left(\cup_{p \in P}\left|M_{p}\right|\right)<\kappa$, logo \# $\left(<\cup_{p \in P}\left|M_{p}\right|>\right)<\kappa$; portanto segue a afirmação .

. $\forall M: S \rightarrow U_{0}-S E T \forall s \in S_{0}\left(M(s)=\cup\left\{N(s): N \in S u b(M)^{<\kappa}\right\}\right)$.

Pois $\forall x \in M(s)\left(<(x, s)>\in S u b(M)^{<\kappa}\right)$.

- $\forall M: S \rightarrow U_{0}-S E T$ ( $M$ é $\mathcal{I}_{\kappa}$-limite indutivo de funtores de cardinais $<\kappa$ (" $\kappa$ pequenos") ) .

Como $I(M, \kappa)$; a categoria associada a $\left(S u b(M)^{<\kappa}: \leq\right)$, é $\kappa$-filtrada e está praticamente em $U_{0}$ e limites indutivos em [S, $\left.U_{0}-S E T\right]$ indexados por categorias (praticamente) em $U_{0}$ são pontuais o funtor "inclusão" $F: I(M, \kappa) \mapsto\left[S, U_{0}-S E T\right]$ é tal que $\underset{I(M, \kappa)}{\lim } \underset{(M)}{\longrightarrow} F(i)=M$.

- A subcategoria plena de $\left[S, U_{0}-S E T\right]$ dos funtores $\kappa$-pequenos está essencialmente em $U_{0}$.

Pois seu esqueleto é (isomorfo a) subcategoria plena de $\left[S, U_{0}-S E T\right]$ com objetos os funtores (automaticamente $\kappa$-pequenos) tais que $\forall s \in S_{0}(M(s)$ é um cardinal $\kappa$-pequeno ) e esta é uma categoria praticamente em $U_{0}$ ( tem cardinal $\leq\left(\left(\kappa^{\kappa} \cdot \kappa^{\kappa^{\kappa}}\right)+\left(\kappa^{\kappa} \cdot \kappa^{\kappa^{\kappa}}\right)^{2} \cdot\left(\kappa \cdot \kappa^{\kappa}\right)\right)=$ $\left.\kappa^{\kappa^{\kappa}}<\theta_{0}\right)$. 
- plena $\left(\left\{M: S \rightarrow U_{0}-S E T: \# M<\kappa\right\}\right) \hookrightarrow\left[S, U_{0}-S E T\right]$ é $\mathcal{I}_{\kappa}$-ind completamento forte de categoria essencialmente em $U_{0}$.

Temos ainda que:

. $\left[S, U_{0}-S E T\right]_{\kappa}=\left\{M: S \rightarrow U_{0}-S E T: \# M<\kappa\right\}$.

Pois $\left[S, U_{0}-S^{\prime} E T\right]_{\kappa}=\left[\left\{M: S \rightarrow U_{0}-S E T: \# M<\kappa\right\}\right] \stackrel{\{\mathbf{E}\}-\lim }{\rightarrow}=\left\{M: S \rightarrow U_{0}-S E T:\right.$ $\# M<\kappa\}$.

Nos exemplos acima temos categorias que são $\kappa$-acessíveis a partir de algum nível $\kappa_{0}$

( respectivamente : $\omega, \omega, \min \left\{\kappa^{\prime}: \omega \leq \kappa^{\prime}=r \epsilon g\left(\kappa^{\prime}\right)<\theta_{0} \wedge \# S<\kappa^{\prime}\right\}$ ). Estes são casos atípicos; em geral temos algo mais fraco mas suficientemente "bom".

Definição 2: Seja $\unlhd(\triangleleft)$ a relação binária em $\{\lambda \in C A R D: \omega \leq \lambda=r \epsilon g(\lambda)\}$ tal que $\kappa \unlhd \kappa^{\prime}\left(\kappa \triangleleft \kappa^{\prime}\right) \Longleftrightarrow$

. $\kappa \leq \kappa^{\prime}\left(\kappa<\kappa^{\prime}\right)$;

. $\forall x\left(\# x<\kappa^{\prime} \Rightarrow \exists \mathcal{A} \in\left[[x]^{<\kappa}\right]^{<\kappa^{\prime}}\left((\mathcal{A}, \subseteq) \hookrightarrow\left([x]^{<\kappa}, \subseteq\right)\right)\right.$ é "funtor" final $\left.)\right)$.

\section{Observação 2 :}

(i) Na definição acima podemos assumir que $x \in\left\{\lambda \in C A R D: \omega \leq \lambda=\operatorname{reg}(\lambda)<\kappa^{\prime}\right\}$. logo para decidir se $\kappa \unlhd \kappa^{\prime}$ basta considerarmos $x \in U$ onde $U$ é um universo tal que $\kappa^{\prime} \in U$

(ii) $\forall \omega \leq \kappa=r \epsilon g(\kappa)(\omega \unlhd \kappa)$.

(iii) $\forall \omega \leq \kappa=r \epsilon g(\kappa)\left(\kappa \triangleleft \kappa^{+}\right)\left(\right.$notemos que $\left.\forall U \in U N I V\left(\kappa \in U \Longleftrightarrow \kappa^{+} \in U\right)\right)$.

(iv) $\forall \omega \leq c f(\mu) \leq \lambda<\mu\left(\lambda^{+} \not \mu^{+}\right)$( por exemplo : $\forall U \in U N I V\left(\aleph_{1} \not \aleph_{\omega+1}<\# U\right.$ ) ) .

Proposição 2: $\forall U(U \in U N I V \vee U=V)((\{\lambda \in C A R D: \omega \leq \lambda=r \epsilon g(\lambda)<\# U$ \}: $\unlhd$ ) é $U$-classe (própria) pré-ordenada \#U-dirigida ) .

(i) $\unlhd$ é reflexiva.

Pois $\kappa \leq \kappa$ e $\forall x\left(\# x<\kappa \Rightarrow\left([x]^{<\kappa}: \subseteq\right)=(\mathcal{P}(x), \subseteq)\right.$ tem máximo $\left.(=x)\right)$.

(ii) $\unlhd$ é transitiva.

Sejam $\kappa \unlhd \kappa^{\prime} \unlhd \kappa^{\prime \prime}$. Para cada $x$ com $\# x<\kappa^{\prime \prime}$ seja $\mathcal{A} \in\left[[x]^{<\kappa^{\prime}}\right]^{<\kappa^{\prime \prime}}$ tal que $\mathcal{A}$ é cofinal em $[x]^{<\kappa^{\prime}}$ então $\forall y \in \mathcal{A} \exists \mathcal{B}_{y} \in\left[[y]^{<\kappa}\right]^{<\kappa^{\prime}}$ tal que $\mathcal{B}_{y}$ é cofinal em $[y]^{<\kappa}$, então $\mathcal{B}=\cup_{y \in \mathcal{A}} \mathcal{B}_{y}$ é tal que:

. $\mathcal{B} \in\left[[x]^{<\kappa}\right]^{<\kappa^{\prime \prime}}$

Pois $\kappa^{\prime \prime}$ é regular.

. $\mathcal{B}$ é cofinal em $[x]^{<\kappa}$.

Pois $\forall w \in[x]^{<\kappa} \exists y \in \mathcal{A}(w \subseteq y \subseteq x) \operatorname{logo} \exists z \in \mathcal{B}_{y} \subseteq \mathcal{B}(w \subseteq z \subseteq y \subseteq x)$.

(iii)

. $\forall \kappa, \kappa^{\prime} \subseteq\{\lambda \in C A R D: \omega \leq \lambda=r \epsilon g(\lambda)<\# U\}\left(\kappa<\kappa^{\prime} \wedge \forall \omega \leq \mu<\kappa \forall \omega \leq \mu^{\prime}<\kappa^{\prime}\right.$ $\left.\left(\left(\mu^{\prime}\right)^{\mu}<\kappa^{\prime}\right) \Rightarrow \kappa \triangleleft \kappa^{\prime}\right)$. 

é cofinal.

Pois $\forall \omega \leq \mu^{\prime}<\kappa^{\prime}\left(\#\left[\mu^{\prime}\right]^{<\kappa}=\sum_{\omega \leq \mu<\kappa}\left(\mu^{\prime}\right)^{\mu}<\kappa^{\prime}\right) \log 0\left[\mu^{\prime}\right]^{<\kappa} \in\left[\left[\mu^{\prime}\right]^{<\kappa}\right]^{<\kappa^{\prime}}$ e $\left[\mu^{\prime}\right]^{<\kappa} \hookrightarrow\left[\mu^{\prime}\right]^{<\kappa}$ . $\forall \kappa, \kappa^{\prime \prime} \subseteq\left\{\lambda \in C^{\prime} A R D: \omega \leq \lambda=r \in g(\lambda)<\# U\right\}\left(\kappa<\kappa^{\prime \prime} \Rightarrow\left(\kappa \triangleleft\left(2^{<\kappa^{\prime \prime}}\right)^{+}<\# U\right)\right.$.

Pois \#U é inacessivel $\vee \# U=\infty$ e se tomamos $\kappa^{\prime}=\left(2^{<\kappa^{\prime \prime}}\right)^{+}$temos:

(a) $\kappa<2^{\kappa} \leq 2^{<\kappa^{\prime \prime}}<\kappa^{\prime}$.

(b) $\forall \omega \leq \mu<\kappa \forall \omega \leq \mu^{\prime}<\kappa^{\prime}\left(\left(\mu^{\prime}\right)^{\mu} \leq\left(2^{<\kappa^{\prime \prime}}\right)^{\mu}=2^{<\kappa^{\prime \prime}}<\kappa^{\prime}\right)$.

. $\forall \Xi \subseteq\{\lambda \in C A R D: \omega \leq \lambda=r \in g(\lambda)<\# U\}\left(\# \Xi<\# U \Rightarrow \exists \kappa_{0}<\# U \forall \kappa \in \Xi\right.$ ( $\left.\kappa \triangleleft \kappa_{0}\right)$.

Pois $\forall \kappa \in \Xi\left(\kappa<\kappa^{\prime \prime}=(\bigvee \Xi)^{+}<\# U\right)$.

A seguir sempre tomaremos as restrições de $\triangleleft$ : $\unlhd$ ao universo $U_{0}$.

Proposição 3 : Sejam $\kappa, \kappa^{\prime}$ cardinais regulares infinitos $U_{0}$-pequenos. $I \in$ obj $\left(U_{0}-C A T\right)$ : $\Xi\left(I,<\kappa^{\prime}\right)=\left\{X \in \operatorname{obj}\left(U_{0}-C A T\right): X\right.$ é subcategoria plena de $\left.I \wedge \# X<\kappa^{\prime}\right\}$ : $\Xi\left(I,<\kappa^{\prime}, \mathcal{I}_{\kappa}\right)=\Xi\left(I,<\kappa^{\prime}\right) \cap \mathcal{I}_{k}$. Se $I \in \mathcal{I}_{\kappa}$ e $\kappa \triangleleft \kappa^{\prime}$ então $\Xi\left(I,<\kappa^{\prime}, \mathcal{I}_{\kappa}\right)$ é cofinal em $\Xi\left(I:<\kappa^{\prime}\right)$ (ordenados por inclusão) .

Dado $X \in \Xi\left(I,<\kappa^{\prime}\right)$ definiremos por indução em $\alpha \in \kappa+1=[0, \kappa]$ uma cadeia de subcategorias plenas de $I$ que contém $X$ e tais que $\left\{X_{\alpha}\right\}_{\alpha \in \kappa+1} \subseteq \Xi\left(I,<\kappa^{\prime}\right)$.

. $\alpha=0 \Rightarrow X_{0}=X$

Satisfaz as condições acima.

. $\alpha=\cup \alpha \neq 0 \Rightarrow \operatorname{obj}\left(X_{\alpha}\right)=\cup_{\beta<\alpha} \operatorname{obj}\left(X_{\beta}\right) \wedge \operatorname{mor}\left(X_{\alpha}\right)=\cup_{\beta<\alpha} \operatorname{mor}\left(X_{\beta}\right)$.

$X_{\alpha}$ satisfaz as condições pela hipótese de indução, porque $\alpha \leq \kappa<\kappa^{\prime}$ e $\kappa^{\prime}$ é regular e porque a "reunião $\omega$-dirigida" de subcategorias plenas de uma categoria determina subcategoria plena (é o limite indutivo do diagrama associado !) .

$\exists \beta(\alpha=\beta+1) \Rightarrow$ como $\kappa \triangleleft \kappa^{\prime}$, pela hipótese de indução temos que $\exists \Sigma_{\beta} \in$ $\left[\left[\operatorname{mor}\left(X_{\beta}\right)\right]^{<\kappa}\right]^{<\kappa^{\prime}}$ cofinal, logo tomando as subcategorias plenas geradas pelos morfismos de cada elemento de $\Sigma_{\beta}$ obtemos $\Psi_{\beta} \in\left[\Xi\left(X_{\beta},<\kappa\right)\right]^{<\kappa^{\prime}}$ cofinal. Para cada $W \in \Psi_{\beta}$ escolhemos $\eta_{W}$ : um cone indutivo comutativo em $I \in \mathcal{I}_{\kappa}$ : com base $j_{W}: W \hookrightarrow X_{\beta} \hookrightarrow I$. Definimos então $X_{\alpha}$ como a subcategoria plena de $I$ gerada pelo conjunto de morfismos $\operatorname{mor}\left(X_{\beta}\right) \cup$ $X(\beta, \Sigma, \eta)$ onde $X(\beta, \Sigma, \eta)=\left\{\eta_{W}\left(\iota_{w}\right): W \in \Psi_{\beta} \wedge w \in W_{0}\right\}$.

A hipótese de indução se mantém pois $\# \operatorname{mor}\left(X_{\beta}\right)<\kappa^{\prime}: \# \Psi_{\beta}<\kappa^{\prime}, \forall W \in \Psi_{\beta}$ ( $\#$ obj $\left.(W)<\kappa<\kappa^{\prime}\right)$ e porque $\kappa^{\prime}$ é regular.

. $X \hookrightarrow X_{\kappa} \in \Xi\left(I .<\kappa^{\prime}, I_{k}\right)$.

Sejam $P \in \operatorname{obj}\left(U_{0}-C A T\right)$ tal que $\# P<\kappa$ e $\Gamma: P \rightarrow X_{\kappa}$. Como $\kappa$ é regular. $\exists \beta<\kappa\left(\left(P \stackrel{\Gamma}{\rightarrow} X_{\kappa}\right)=\left(P \stackrel{\Gamma_{\beta}}{\rightarrow} \mathrm{X}_{\beta} \hookrightarrow X_{\kappa}\right)\right)$ : como $\# P<\kappa$ temos que $\exists W \in \Psi_{\beta}($ $\left.\left(P \stackrel{\Gamma_{\beta}}{\rightarrow} X_{\beta}\right)=\left(P \stackrel{\Gamma_{W}}{\rightarrow} W \hookrightarrow X_{\beta}\right)\right)$ logo $\eta_{W}$ determina um cone indutivo comutativo com base $\left(P \stackrel{\Gamma_{W}}{\rightarrow} W \hookrightarrow X_{\beta} \hookrightarrow X_{\beta+1}\right)$ e como $\kappa$ é ordinal limite, obtemos cone indutivo comutativo( 
em $\left.X_{\kappa}\right)$ de base $\Gamma$.

Corolário : Nas condições da proposição anterior temos :

(i) $\left(\Xi\left(I,<\kappa^{\prime}, \mathcal{I}_{\kappa}\right)\right.$ : $\left.\hookrightarrow\right)$ é $U_{0}$-conjunto ordenado $\kappa^{\prime}$-dirigido.

(ii) $\operatorname{obj}(I)=\cup\left\{\operatorname{obj}(X): X \in \Xi\left(I,<\kappa^{\prime}, \mathcal{I}_{\kappa}\right)\right\} \wedge \operatorname{mor}(I)=\cup\{\operatorname{mor}(X): X \in \Xi(I,<$ $\left.\left.\kappa^{\prime}, \mathcal{I}_{\kappa}\right)\right\}$.

(i) Como $I \in \operatorname{obj}\left(U_{0}-C A T\right)$ temos $\left(\Xi\left(I,<\kappa^{\prime}, \mathcal{I}_{\kappa}\right)\right.$; $\left.\hookrightarrow\right)$ é $U_{0}$-conjunto ordenado ; e é $\kappa^{\prime}$-dirigido pois $\kappa^{\prime}$ é regular e $\Xi\left(I,<\kappa^{\prime}, \mathcal{I}_{\kappa}\right)$ é cofinal em $\Xi\left(I:<\kappa^{\prime}\right)$.

(ii) Porque $\operatorname{obj}(I)=\cup\left\{\operatorname{obj}(X): X \in \Xi\left(I,<\kappa^{\prime}\right)\right\} \wedge \operatorname{mor}(I)=\cup\{\operatorname{mor}(X): X \in \Xi(I,<$ $\left.\left.\kappa^{\prime}\right)\right\}$ e $\Xi\left(I,<\kappa^{\prime}, \mathcal{I}_{\kappa}\right)$ é cofinal em $\Xi\left(I,<\kappa^{\prime}\right)$.

Proposição 4: $\forall \kappa \triangleleft \kappa^{\prime} \forall A\left(A \kappa\right.$-acessível $\Rightarrow A \kappa^{\prime}$-acessível $)$.

. A é $U_{1}$-categoria $\mathcal{I}_{\kappa^{\prime}}$-ind completa.

Pois $\kappa \leq \kappa^{\prime} \Rightarrow \mathcal{I}_{\kappa^{\prime}} \subseteq \mathcal{I}_{\kappa}$ e $J_{\kappa}: A_{\kappa} \hookrightarrow A$ é $\mathcal{I}_{\kappa}$-ind completamento forte de $A_{\kappa}$ (com $A_{\kappa}$ essencialmente em $U_{0}$ ) .

Seja $C$ a subcategoria plena de $A$ tal que $C_{0}=\left\{a \in A_{0}: \exists I \in \mathcal{I}_{\kappa} \exists H: I \rightarrow A_{\kappa}\right.$ ( $\left.\# I<\kappa^{\prime} \wedge a \cong \underset{I}{\simeq} \lim _{\longrightarrow} J_{\kappa} \circ H\right\}$; temos então:

. $C \subseteq A_{\kappa^{\prime}}$.

Pois $\kappa \leq \kappa^{\prime} \Rightarrow\left(\mathcal{I}_{\kappa^{\prime}} \subseteq \mathcal{I}_{\kappa} \wedge A_{\kappa} \subseteq A_{\kappa^{\prime}}\right)$.

. $C$ está essencialmente em $U_{0}$.

Pois $A_{\kappa}$ está essencialmente em $U_{0}$ e existem $<\theta_{0}$ categorias duas a duas não isomorfas com cardinal $<\kappa^{\prime}<\theta_{0}$.

. $\forall a \in A_{0} \exists I^{\prime} \in \mathcal{I}_{\kappa^{\prime}} \exists H^{\prime}: I^{\prime} \rightarrow C\left(a \cong \underset{I^{\prime}}{\lim } J^{\prime} \circ H^{\prime}\right)$ onde $J^{\prime}: C \hookrightarrow A$.

- $\forall a \in A_{0} \operatorname{sejam} I \in \mathcal{I}_{\kappa}$ e $H: I \rightarrow A_{\kappa}$ tais que $a \cong \underset{I}{\lim } J_{\kappa} \circ H$. Consideremos $\left(\Xi\left(I,<\kappa^{\prime}, \mathcal{I}_{\kappa}\right) ; \hookrightarrow\right)$ como na proposição anterior. Pela proposição 1 em (1.3.1) e o corolário anterior temos $\underset{\Xi\left(I ;<\kappa^{\prime}, \mathcal{I}_{\kappa}\right)}{\lim _{X}} \lim _{\boldsymbol{X}} J_{\kappa} \circ H_{\mid X} \cong \underset{I}{\cong} \lim _{\kappa} \circ H \cong a$; basta notar que $\forall X \in \Xi\left(I,<\kappa^{\prime}, \mathcal{I}_{\kappa}\right)$ $\left(\left.\underset{X}{\lim } J_{\kappa} \circ H\right|_{X} \in \operatorname{obj}(C)\right)$.

. $J^{\prime}: C \hookrightarrow A$ é $\mathcal{I}_{\kappa^{\prime}}$-ind completamento forte de $C$ e $C$ é categoria essencialmente em $U_{0}$.

Corolário : Seja $A$ categoria $\kappa_{0}$-acessível para algum cardinal regular infinito $\kappa_{0}<\theta_{0}$. Temos que :

(i) $\forall X$ subcategoria plena de $A$ essencialmente em $U_{0} \exists \kappa\left(A\right.$ é $\kappa$-acessível $\left.\wedge X \hookrightarrow A_{\kappa}\right)$.

(ii) $\forall \kappa\left(A_{\kappa}\right.$ é $U_{1}$-categoria essencialmente em $\left.U_{0}\right)$. 
(iii) $A=\underset{k<\theta_{0}}{\lim } A_{k}$.

(i) Escolhemos para cada $a \in \mathrm{X}_{0}$ um diagrama $H_{a}: I_{n} \rightarrow A_{\kappa_{0}}$ tal que $I_{a} \in \mathcal{I}_{\kappa_{0}}$ e $a \cong \underset{I_{a}}{\lim } J_{\kappa_{0}} \circ H_{a}$. Consideramos para cada $a \in X_{0} \kappa_{a}=\left(\kappa_{0}+\# I_{a}\right)^{+}$. então $\forall a \in X_{0}$ ( $\left.a \in A_{\kappa_{a}}\right)$. Como $X$ está essencialmente em $U_{0}$ temos $\kappa^{\prime}=\left(\sup \left\{\kappa_{a}: a \in X_{0}\right\}\right)^{+}<\theta_{0} \mathrm{e}$ $\operatorname{assim} X \hookrightarrow A_{\kappa^{\prime}}$. Escolhemos, pela proposição 2, $\kappa$ tal que $\kappa^{\prime} \triangleleft \kappa$ e $\kappa_{0} \triangleleft \kappa, \operatorname{logo} X \hookrightarrow A_{\kappa}$ e. pela proposição anterior, $A$ é $\kappa$-acessivel.

(ii) Para cada $\kappa<\theta_{0}$ cardinal regular infinito existe $\kappa^{\prime}$ tal que $\kappa \triangleleft \kappa^{\prime}$ e $\kappa_{0} \triangleleft \kappa^{\prime}$. Assim $A_{\kappa} \hookrightarrow A_{\kappa^{\prime}}$ e $A_{\kappa^{\prime}}$ está essencialmente em $U_{0}$. O resultado segue pois $A_{\kappa}$ é fechada por isomorfismos .

(iii) Pois por (i) e (ii) temos $\operatorname{obj}(A)=\cup_{\kappa<\theta_{0}} A_{\kappa}$ e está é uma "reunião dirigida" de subcategorias plenas de $A$.

\section{Observação 3 :}

(i) A relação $\unlhd$ é a mais geral possível, isto é :

$\forall \kappa, \kappa^{\prime}<\theta_{0}$ cardinais regulares infinitos $\left(\forall A \in \operatorname{obj}\left(U_{1}-C A T\right)(A \kappa\right.$-acessível $\Rightarrow A$ $\kappa^{\prime}$-acessivel ) $\Rightarrow \kappa \unlhd \kappa^{\prime}$ ) .

(ii) Nas condições da proposição 4 segue que $A_{\kappa^{\prime}}=[C] \stackrel{\{\mathbf{E}\}-\lim }{\rightarrow}$, mas $A_{\kappa^{\prime}}=C$ ocorre.

(iii) Dados $\kappa, \kappa^{\prime \prime}<\theta_{0}$ cardinais regulares infinitos : $A, A^{\prime}, A^{\prime \prime} U_{1}$-categorias e $F$; $F^{\prime \prime}$ funtores tais que $F \in \kappa-A C E\left(A, A^{\prime}\right)$ e $F^{\prime \prime} \in \kappa^{\prime \prime}-A C E\left(A^{\prime}, A^{\prime \prime}\right)$. Como existe $\kappa_{0}$ tal que $\kappa \triangleleft \kappa_{0}$ e $\kappa^{\prime \prime} \triangleleft \kappa_{0}$ temos $F^{\prime \prime} \circ F \in \kappa_{0}-A C E\left(A, A^{\prime \prime}\right)$ e assim faz sentido a:

\section{Definição 3 :}

(i) $A C E$ é a subcategoria de $U_{1}-C A T$ tal que $\operatorname{obj}(A C E)=\cup\left\{\operatorname{obj}(\kappa-A C E): \kappa<\theta_{0}\right.$ é cardinal regular infinito $\} ; \operatorname{mor}(A C E)=\cup\left\{\operatorname{mor}(\kappa-A C E): \kappa<\theta_{0}\right.$ é cardinal regular infinito $\}$.

(ii) $\mathcal{A C E}$ é a sub-2-categoria localmente plena de $U_{1}-\mathcal{C} \mathcal{A} \mathcal{T}$ com categoria ordinária subjacente $A C E$.

Proposição 5 : Princípio da uniformização : Dados $X U_{1^{-}}$categoria essencialmente em $U_{0}$ e $\Gamma \in U_{2}-C A T(X, A C E)$ existe $\kappa<\theta_{0}$ cardinal regular infinito tal que:

(i) $(X \stackrel{\Gamma}{\rightarrow} A C E)=(X \stackrel{\bar{\Gamma}}{\rightarrow} \kappa-A C E \hookrightarrow A C E)$.

(ii) $\forall x \stackrel{f}{\rightarrow} x^{\prime}\left(\left((\Gamma(f))_{0}\right)_{*}\left(\Gamma(x)_{\kappa}\right) \subseteq \Gamma\left(x^{\prime}\right)_{\kappa}\right)$.

Escolhemos; para cada $x \in X_{0}, \kappa_{x}$ tal que $\Gamma(x) \in o b j\left(\kappa_{x}-A C E\right)$ e para cada $f \in X_{1}$ : $\kappa_{f}$ tal que $\Gamma(f) \in \operatorname{mor}\left(\kappa_{f}-A C E\right)$. Como $X$ está essencialmente em $U_{0}$ podemos supor

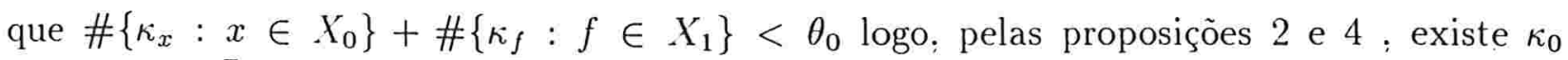
tal que $(X \stackrel{\Gamma}{\rightarrow} A C E)=\left(X \stackrel{\Gamma_{0}}{\rightarrow} \kappa_{0}-A C E \hookrightarrow A C E\right)$. Em particular. $\forall x \in X_{0}\left(\Gamma(x)_{\kappa_{0}}\right.$ 
é $U_{1}$-categoria essencialmente em $\left.U_{0}\right)$, portanto, raciocinando como acima, existe $\kappa$ com $\kappa_{0} \triangleleft \kappa$ e tal que $\forall x \stackrel{f}{\rightarrow} x^{\prime} \in X\left(\left((\Gamma(f))_{0}\right)_{*}\left(\Gamma(x)_{\kappa_{0}}\right) \subseteq \Gamma\left(x^{\prime}\right)_{\kappa}\right)$. Pela proposição 2 temos $(X \stackrel{\Gamma}{\rightarrow} A C E)=(X \stackrel{\bar{\Gamma}}{\rightarrow} \kappa-A C E \hookrightarrow A C E)$ e pela observação 3.(ii) $\forall x \stackrel{f}{\rightarrow} x^{\prime} \in X($ $\left((\Gamma(f))_{0}\right)_{*}\left(\Gamma(x)_{\kappa}\right)=\left((\Gamma(f))_{0}\right)_{*}\left(\left\{a \in \operatorname{obj}(\Gamma(x)): \exists I \in \mathcal{I}_{\kappa_{0}} \exists H: I \rightarrow \Gamma(x)_{\kappa_{0}}(\# I<\kappa)\right.\right.$ $\left.\left.\exists p: a \rightarrow \underset{I}{\lim } J_{\left(x, \kappa_{0}\right)} \circ H \underset{I}{\underset{I}{:}} J_{\left(x, \kappa_{0}\right)} \circ H \rightarrow a\left(p \circ i=1_{a}\right)\right\}\right) \subseteq\left\{a^{\prime} \in \operatorname{obj}\left(\Gamma\left(x^{\prime}\right)\right): \exists I \in \mathcal{I}_{\kappa_{0}}\right.$ $\exists H^{\prime}: I \rightarrow \Gamma\left(x^{\prime}\right)_{\kappa}(\# I<\kappa)^{I} \exists p^{\prime}: a^{\prime} \underset{I}{\rightarrow} \underset{I}{\lim } J_{(x, \kappa)} \circ H^{\prime} \exists i^{\prime}: \underset{I}{\lim } J_{(x, \kappa)} \circ H^{\prime} \rightarrow a^{\prime}\left(p^{\prime} \circ i^{\prime}=1_{a^{\prime}}\right)$ \}$=\left\{a^{\prime} \in \operatorname{obj}\left(\Gamma\left(x^{\prime}\right)\right): \exists I \in \mathcal{I}_{\kappa_{0}} \exists H^{\prime}: I \rightarrow \Gamma\left(x^{\prime}\right)_{\kappa}\left(\# I<\kappa \wedge a^{\prime} \cong \underset{I}{\lim } J_{(x, \kappa)} \circ H^{\prime}\right)\right\} \subseteq \Gamma\left(x^{\prime}\right)_{\kappa}$

Observação 4 : Existe uma $U$-classe própria de cardinais $\kappa$ satisfazendo (i) e (ii) acima.

Proposição 6 :

(i) $\forall A \in \operatorname{obj}(A C E) \forall F \in U_{1}-C A T\left(A, U_{0}-S E T\right)\left(F \in A C E\left(A, U_{0}-S E T\right) \Longleftrightarrow\right.$ $\left.\exists X \in \operatorname{obj}\left(U_{0}-C A T\right) \exists \Gamma: X \rightarrow A^{\star}\left(F \cong \underset{X}{\lim } Y_{A^{*}} \circ \Gamma(x)\right)\right)$.

(ii) $\forall A, A^{\prime} \subseteq \operatorname{obj}(A C E) \forall F \in U_{1}-C A T\left(A, A^{\prime}\right)\left(F \in A C E\left(A, A^{\prime}\right) \Longleftrightarrow \forall a^{\prime} \in A_{0}^{\prime}\right.$ ( $\left.A^{\prime}\left(a^{\prime} ;-\right) \circ F \in A C E\left(A, U_{0}-S E T\right)\right)$.

(i)

\section{$(\Rightarrow)$}

Escolhemos, por uniformidade, $\kappa$ tal que $F \in \kappa-A C E\left(A, U_{0}-S E T\right)$. Consideramos $C \in \operatorname{obj}\left(U_{0}-C A T\right)$ e $J: C \rightarrow A \mathcal{I}_{\kappa}$-ind completamento forte de $A$. $J$ é, em particular, $\mathcal{I}_{\kappa}$-ind completamento fraco de $C$, portanto, $\left(L_{J}\right.$; $\left[J_{0} U_{0}-S E T\right]$ I: $\left.\eta, \epsilon\right): U_{1}-C A T\left(C, U_{0}-\right.$

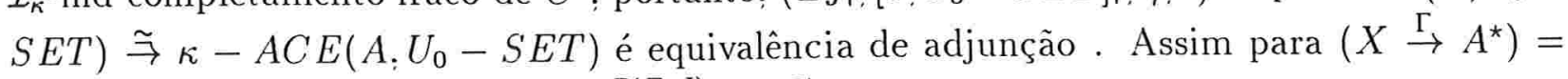
$\left((K(F \circ J))^{\star} \cong\left(Y_{C^{*}} \rightarrow(F \circ J)\right) \stackrel{P(F \circ J)}{\rightarrow} C^{\star} \stackrel{J^{*}}{\rightarrow} A^{\star}\right) \operatorname{temos}(K(F \circ J))^{\star} \in \operatorname{obj}\left(U_{0}-C A T\right)$

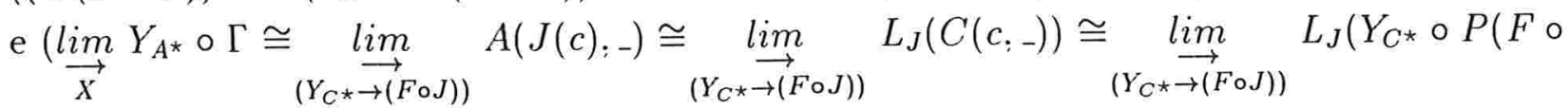
$\left.J)) \cong L_{J}\left(\underset{\left(Y_{\left.C^{\star} \rightarrow(F \circ J)\right)}\right.}{\lim _{\longrightarrow}} Y_{C^{\star}} \circ P(F \circ J)\right) \cong L_{J}(F \circ J) \stackrel{\epsilon_{F} \cong}{\rightarrow} F\right)$.

$(\Leftarrow)$

Como $A$ é $\kappa_{0}$-acessível para algum $\kappa_{0}$; podemos escolher, para cada $x \in X_{0} ; \kappa_{x}$ tal que $\Gamma(x) \in A_{k_{x}}$. Porque $X \in \operatorname{obj}\left(U_{0}-C A T\right)$ temos $\#\left\{\kappa_{x}: x \in X_{0}\right\}<\theta_{0}$ logo podemos escolher $\kappa$ tal que $\forall x \in X_{0}\left(\kappa_{x}<\kappa\right)$ e $\kappa_{0} \triangleleft \kappa$. Assim $A$ é $\kappa$-acessivel e $F$ é limite indutivo de funtores que preservam $\mathcal{I}_{\kappa}$-limites indutivos e; como limites indutivos comutam com limites indutivos, $F \in \kappa-A C E\left(A, U_{0}-S E T\right)$.

(ii)

$$
(\Rightarrow)
$$

Como obj $\left(A^{\prime}\right)=\cup_{\kappa<\theta_{0}} A_{\kappa}^{\prime}$ temos $\forall a^{\prime} \in A^{\prime}\left(A^{\prime}\left(a^{\prime} ;-\right) \circ F \in A C E\left(A, U_{0}-S E T\right)\right)$. 


\section{$(\Leftarrow)$}

Seja $\kappa^{\prime}$ tal que $A^{\prime}$ seja $\kappa^{\prime}$-acessível. Como $A_{\kappa^{\prime}}^{\prime}$ está essencialmente em $U_{0}$ existe $\kappa_{0}>\kappa^{\prime}$ tal que $\forall a^{\prime} \in A_{\kappa^{\prime}}^{\prime}\left(A^{\prime}\left(a^{\prime},{ }_{-}\right) \circ F \in \kappa_{0}-A C E\left(A, U_{0}-S E T\right) \wedge A^{\prime}\left(a^{\prime} ;-\right) \in \kappa_{0}-A C E\left(A^{\prime}, U_{0}-S E T\right)\right.$ ). como $i_{\kappa^{\prime}}: A_{\kappa^{\prime}}^{\prime} \hookrightarrow A^{\prime}$ é denso, o funtor $\tilde{i}_{\kappa^{\prime}}: A^{\prime} \rightarrow\left[\left(A_{\kappa^{\prime}}^{\prime}\right)^{\star}, U_{0}-S E T\right]$ é hom-bijetor: logo reflete isomorfismos. Assim $\tilde{i}_{\kappa^{\prime}} \circ F$ preserva $\mathcal{I}_{\kappa_{0}}$-limites indutivos, $\tilde{i}_{\kappa^{\prime}}$ preserva $\mathcal{I}_{\kappa_{0}}$-limites indutivos e reflete isomorfismos, portanto $F$ preserva $\mathcal{I}_{\kappa_{0}}$-limites indutivos. 


\section{Capítulo 3}

\section{Esboços}

Tradicionalmente em Matemática, muitas estruturas (conjuntistas) são especificadas através de teorias de primeira ordem. Esboços , por sua vez, são "sintaxes" cuja "semântica pretendida" são as categorias de estruturas descritas por limites projetivos/indutivos, estruturadas sobre alguma categoria de base.

Referências principais : [029], [096] .

\section{Definição 1 :}

(i) $\mathcal{S}$ é um esboço gráfico (g-esboço ) $\Longleftrightarrow \mathcal{S}=(S, D, c P, c I)$ onde $S \in(G R A)_{0}, D \in$ $\mathcal{P}\left(\cup_{G \in(G R A)_{0}} G R A(G, S)\right), c P \in \mathcal{P}\left(\cup_{G \in(G R A)_{0}} G R A\left(G^{-}, S\right)\right), c I \in \mathcal{P}\left(\cup_{G \in(G R A)_{0}} G R A\left(G^{+}, S\right)\right)$

$$
\begin{aligned}
& \mathcal{D}(\mathcal{S})=\left\{G \in(G R A)_{0}: \exists d: G \rightarrow S \in D\right\} . \\
& . \mathcal{P}(\mathcal{S})=\left\{G \in(G R A)_{0}: \exists p: G^{-} \rightarrow S \in c P\right\} . \\
& . \mathcal{I}(\mathcal{S})=\left\{G \in(G R A)_{0}: \exists i: G^{+} \rightarrow S \in c I\right\} \\
& . \delta(\mathcal{S})=\min \{\delta: \omega \leq \delta \wedge \forall G \in \mathcal{D}(\mathcal{S})(\# G \leq \delta)\} . \\
& . \pi(\mathcal{S})=\min \{\pi: \omega \leq \pi=r \epsilon g(\pi) \wedge \forall G \in \mathcal{P}(\mathcal{S})(\# G<\pi)\} \\
& . \iota(\mathcal{S})=\min \{\iota: \omega \leq \iota \wedge \forall G \in \mathcal{I}(\mathcal{S})(\# G \leq \iota)\} . \\
& . \tau(\mathcal{S})=\operatorname{má}\{\# S ; \# D, \# P ; \# I ; \delta(\mathcal{S}), \pi(\mathcal{S}), \iota(\mathcal{S})\} . \\
& . \kappa(\mathcal{S})=\left(\tau(\mathcal{S})^{<\pi(\mathcal{S})}\right)^{+} .
\end{aligned}
$$

Os cardinais acima são denominados cardinais característicos de $\mathcal{S}$.

(ii) $\mathcal{S}$ é um esboço categorial (c-esboço ) $\Longleftrightarrow \mathcal{S}=(S, c P, c I)$ onde $S \in(C A T)_{0}$ : $c P \in \mathcal{P}\left(\cup_{G \in(G R A)_{0}} G R A\left(G^{-}, \mathcal{E}(S)\right)\right), c I \in \mathcal{P}\left(\cup_{G \in(G R A)_{0}} G R A\left(G^{+}, \mathcal{E}(S)\right)\right)$.

. $\mathcal{P}(\mathcal{S})=\left\{G \in(G R A)_{0}: \exists p: G^{-} \rightarrow \mathcal{E}(S) \in c P\right\}$.

$\mathcal{I}(\mathcal{S})=\left\{G \in(G R A)_{0}: \exists i: G^{+} \rightarrow \mathcal{E}(S) \in c I\right\}$.

. $\pi(\mathcal{S})=\min \left\{\pi: \omega \leq \pi=\operatorname{reg}(\pi) \wedge \forall G_{1} \mathcal{P}(\mathcal{S})(\# G<\pi)\right\}$.

. $\iota(\mathcal{S})=\min \{\iota: \omega \leq \iota \wedge \forall G \in \mathcal{I}(\mathcal{S})(\# G \leq \iota)\}$.

. $\tau(\mathcal{S})=\operatorname{máx}\{\# S: \# P$ :\#I, $\pi(\mathcal{S}): \iota(\mathcal{S})\}$. 


$$
\kappa(\mathcal{S})=\left(\tau(\mathcal{S})^{<\pi(\mathcal{S})}\right)^{+}
$$

Os cardinais acima são denominados cardinais característicos de $\mathcal{S}$.

(iii) Sejam $\mathcal{S}=(S, D, c P, c I), \mathcal{S}^{\prime}=\left(D^{\prime}, c P^{\prime}, c I^{\prime}\right)$ g-esboços $. \mathcal{F}: \mathcal{S} \rightarrow \mathcal{S}^{\prime}$ é morfismo de g-esboços $\Longleftrightarrow \mathcal{F}=\left(\mathcal{S}, F, \mathcal{S}^{\prime}\right)$ onde $F \in G R A\left(S, S^{\prime}\right)$ é tal que $\{F \circ d: d \in D\} \subseteq D^{\prime}$ : $\{F \circ p: p \in c P\} \subseteq c P^{\prime}:\{F \circ i: i \in c I\} \subseteq c I^{\prime}$.

Definimos $g-E S Q$; a categoria dos g-esboços , de modo a termos naturalmente um funtor de esquecimento $\mathcal{E}: g-E S Q \rightarrow G R A$.

(iv) Analogamente definimos $c-E S Q$ e $\mathcal{E}: c-E S Q \rightarrow C A T$. Denotamos $c-\mathcal{E S Q}$ a 2-categoria com categoria ordinária subjacente $c-E S Q$ e 2-flechas as "transformações naturais", isto é, $\mathcal{E}$ é a parte ordinária de um 2 -funtor localmente pleno $\overline{\mathcal{E}}: c-\mathcal{E S} \mathcal{Q} \rightarrow \mathcal{C} \mathcal{A} \mathcal{T}$

Observação 1 : " $c-E S Q(g-E S Q)$ é topológica sobre $C A T(G R A)$ ":

(i) Para cada $S \in(C A T)_{0}$ consideremos $c-E S Q_{C}$; a fibra em $c-E S Q$ de $S$; isto é, $c-E S Q_{S}$ é a subcategoria de $c-E S Q$ tal que $\mathcal{E} \mid: c-E S Q_{S} \rightarrow\left(S \stackrel{1_{S}}{\rightarrow} S\right)$. Para cada $F \in C A T\left(S, S^{\prime}\right)$ temos funtores $F_{*}: c-E S Q_{S} \rightarrow c-E S Q_{S^{\prime}}$ e $F^{*}: c-E S Q_{S^{\prime}} \rightarrow c-E S Q_{S}$ tais que: $F_{*}(\mathcal{S})=\left(S^{\prime}:\{F \circ p: p \in c P\}:\{F \circ i: i \in c I\}\right)$ e $F^{*}\left(\mathcal{S}^{\prime}\right)=\left(S:\left\{p: F \circ p \in c P^{\prime}\right\}:\{i:\right.$ $\left.\left.F \circ i \in c I^{\prime}\right\}\right)$.

(ii) $S \neq \emptyset \Rightarrow c-E S Q_{S}$ é uma categoria não pequena associada a um reticulado (grande) tal que toda subclasse limitada superiormente admite supremo (admite todos os ínfimos de subclasses não vazias).

(iii) Temos bijeções naturais em $\mathcal{S} \in\left(c-E S Q_{S}\right)^{\star}$ e $\mathcal{S}^{\prime} \in c-E S Q_{S^{\prime}}: c-E S Q_{S^{\prime}}\left(F_{*}(\mathcal{S}) ; \mathcal{S}^{\prime}\right)$ $\cong\left\{\mathcal{F} \in c-E S Q\left(\mathcal{S}, \mathcal{S}^{\prime}\right): \mathcal{E}(\mathcal{F})=F\right\} \cong c-E S Q_{S}\left(\mathcal{S}, F^{*}\left(\mathcal{S}^{\prime}\right)\right)$

(iv) $\mathcal{E}: c-E S Q \rightarrow C A T$ cria todos os limites indutivos e todos os limites projetivos pequenos : como $C A T$ é ind/proj pequeno completa e os funtores de esquecimento $O B J, M O R: C A T \rightarrow S E T$ criam limites projetivos pequenos e limites indutivos pequenos $\omega$-filtrados : $c-E S Q$ tem propriedades análogas.

\section{Definição 2 :}

(i) Sejam $\mathcal{D}, \mathcal{P} ; \mathcal{I} \subseteq(G R A)_{0} \cdot g-(\mathcal{D}, \mathcal{P}, \mathcal{I})-E S Q$ é a subcategoria plena de $g-E S Q$ dos g-esboços $\mathcal{S}$ tais que $\mathcal{D}(\mathcal{S}) \subseteq \mathcal{D}, \mathcal{P}(\mathcal{S}) \subseteq \mathcal{P}, \mathcal{I}(\mathcal{S}) \subseteq \mathcal{I}$. Analogamente definimos $c-(\mathcal{P}, \mathcal{I})-E S Q$.

(ii) Dado $U \in U N I V g-U-E S Q(c-U-E S Q)$ é a subcategoria plena de $g-E S Q(c-$ $E S Q)$ dos g-esboços $\mathcal{S}=(S ; D ; c P ; c I) \in U$ (c-esboços $\mathcal{S}=(S ; c P ; c I) \in U)$.

(iii) Dados $U \in U N I V$ e $\mathcal{S} \in \operatorname{obj}(g-E S Q)(\mathcal{S} \in \operatorname{obj}(c-E S Q))$. Dizemos que $\mathcal{S}$ está praticamente em $U$ no caso de ser isomorfo a algum g-esboço (c-esboco) em $U$.

(iv) Dados $U \in U N I V$ e $\mathcal{S} \in o_{b j}(c-E S Q)$. Dizemos que $\mathcal{S}$ está essencialmente em $U$ no caso de ser equivalente (na 2-categoria $c-\mathcal{E S Q}$ !) a algum c-esboço em $U$.

Observação 2 : 
(i) Dispomos de análises semelhantes às realizadas acima quando consideramos $c-(\mathcal{P}, \mathcal{I})-$ $E S Q\left(g-(\mathcal{D}, \mathcal{P}, \mathcal{I})-E S Q, c-U-E S Q(g-U-E S Q)\right.$. Notemos que se $S \in(C A T)_{0}-\emptyset$ $\left(S \in(G R A)_{0}-\emptyset\right)$ então a fibra $c-(\mathcal{P}, \mathcal{I})-E S Q_{S}\left(g-(\mathcal{D}, \mathcal{P}: \mathcal{I})-E S Q_{S}\right)$ constitui reticulado pequeno (e completo!) $\Longleftrightarrow \mathcal{P}, \mathcal{I}(\mathcal{D}, \mathcal{P}, \mathcal{I})$ são conjuntos.

(ii) Dadas classes $\mathcal{P} \subseteq \mathcal{P}^{\prime}: \mathcal{I} \subseteq \mathcal{I}^{\prime}$ temos funtores evidentes $c-(\mathcal{P}, \mathcal{I})-E S Q \stackrel{\text { incl }}{\hookrightarrow} c-$ $\left(\mathcal{P}^{\prime}: \mathcal{I}^{\prime}\right)-E S Q \stackrel{\text { rest }}{\rightarrow} c-(\mathcal{P}, \mathcal{I})-E S Q=c-(\mathcal{P} . \mathcal{I})-E S Q \stackrel{i d}{\rightarrow} c-(\mathcal{P} . \mathcal{I})-E S Q$.

(iii) $\mathcal{S}$ praticamente em $g-U-E S Q(c-U-E S Q) \Rightarrow \lambda(\mathcal{S})<\# U \wedge \mu(\mathcal{S})<\# U$.

Definição 3 : Sejam $\mathcal{S}$ um esboço (gráfico ou categorial) $\sigma: \lambda$ cardinais com $\sigma<\propto$ e $\omega \leq \lambda=r \epsilon g(\lambda) \leq \infty$. Disc $c_{\lambda}$ denota a classe de todos os grafos discretos com $<\lambda$ objetos : $P a r_{\sigma}$ é a classe de todos os grafos com dois objetos e $\sigma$ flechas paralelas, com mesma orientação , entre esses.

. $\mathcal{S}$ é trivial $\Longleftrightarrow \mathcal{P}(\mathcal{S})=\mathcal{I}(\mathcal{S})=\emptyset$.

. $\mathcal{S}$ é projetivo $\Longleftrightarrow \mathcal{I}(\mathcal{S})=\emptyset$.

. $\mathcal{S}$ é indutivo $\Longleftrightarrow \mathcal{P}(\mathcal{S})=\emptyset$.

$\mathcal{S}$ é $\lambda$-esboço $\Longleftrightarrow \pi(\mathcal{S}) \leq \lambda$.

. $\mathcal{S}$ é $\lambda$-geométrico $\Longleftrightarrow \mathcal{S}$ é $\lambda$-esboço e $\mathcal{I}(\mathcal{S}) \subseteq D i s c_{\infty} \cup P a r_{2}$.

$\mathcal{S}$ é $\lambda$-local $\Longleftrightarrow \mathcal{S}$ é $\lambda$-esboço e $\mathcal{I}(\mathcal{S}) \subseteq D i s c_{\infty}$.

. $\mathcal{S}$ é $\lambda$-regular $\Longleftrightarrow \mathcal{S}$ é $\lambda$-esboço e $\mathcal{I}(\mathcal{S}) \subseteq \mathrm{Par}_{2}$.

. $\mathcal{S}$ é $\lambda$-coerente $\Longleftrightarrow \mathcal{S}$ é $\lambda$-esboço e $\mathcal{I}(\mathcal{S}) \subseteq D i s c_{\lambda} \cup \operatorname{Par}_{2}$.

. $\mathcal{S}$ é $\lambda$-projetivo $\Longleftrightarrow \mathcal{S}$ é $\lambda$-esboço e $\mathcal{I}(\mathcal{S})=\emptyset$.

. $\mathcal{S}$ é $\lambda$-produto $\Longleftrightarrow \mathcal{P}(\mathcal{S}) \subseteq D i s c_{\lambda}$ e $\mathcal{I}(\mathcal{S})=\emptyset$.

Definição 4 : Sejam $\mathcal{P}: \mathcal{I} \subseteq(G R A)_{0}$

(i) $\mathcal{S}=(S, c P, c I) \in o b j(c-E S Q)$ é c-esboço comutativo $\Longleftrightarrow c P(c I)$ consiste de cones projetivos (indutivos) comutativos. $c c-(\mathcal{P}, \mathcal{I})-E S Q$ é a subcategoria plena de $c-(\mathcal{P}, \mathcal{I})-E S Q$ dos c-esboços comutativos.

(ii) $\mathcal{S}=(S, c P, c I) \in o b j(c-E S Q)$ é c-esboço limite $\Longleftrightarrow c P(c I)$ consiste de cones projetivos (indutivos) limites. $c l-(\mathcal{P}, \mathcal{I})-E S Q$ é a subcategoria plena de $c-(\mathcal{P} ; \mathcal{I})-E S Q$ dos c-esboços limites.

(iii)Suponhamos $\mathcal{P}: \mathcal{I} \in \mathcal{P}\left((G R A)_{0}\right) . \mathcal{S}=(S ; c P, c I) \in \operatorname{obj}(c-E S Q)$ é $(\mathcal{P} ; \mathcal{I})$-teoria $\Longleftrightarrow \mathcal{S}$ é c-esboço limite $\wedge \forall P \in \mathcal{P} \forall F: P \rightarrow S \exists p \in c P($ base $(p)=F) \wedge \forall I \in \mathcal{I} \forall F: I \rightarrow$ $S \exists i \in c I($ base $(i)=F)$. $(\mathcal{P}, \mathcal{I})-T E O$ é a subcategoria plena de $c-E S Q$ das $(\mathcal{P} ; \mathcal{I})$-teorias.

(iv)Suponhamos $\mathcal{P}: \mathcal{I} \in \mathcal{P}\left((G R A)_{0}\right) . \mathcal{S}=(S, c P, c I) \in o b j(c-E S Q)$ é $(\mathcal{P}, \mathcal{I})$-tipo $\Longleftrightarrow \mathcal{S}$ é $(\mathcal{P}, \mathcal{I})$-teoria $\wedge \forall\left\{p, p^{\prime}\right\} \subseteq c\left(\operatorname{base}(p)=\operatorname{bas\epsilon }\left(p^{\prime}\right) \Rightarrow p=p^{\prime}\right) \wedge \forall\left\{i, i^{\prime}\right\} \subseteq c I($ base $(i)=$ $\left.\operatorname{base}\left(i^{\prime}\right) \Rightarrow i=i^{\prime}\right)$. (P, $\left.\mathcal{I}\right)-T Y P$ é a subcategoria plena de $c-E S Q \operatorname{dos}(\mathcal{P}, \mathcal{I})$-tipos.

Para as duas proposições seguintes, consultar o ítem (1.2.2).

Proposição 1 : Sejam $\mathcal{D} \in \mathcal{P}\left((G R A)_{0}\right)$ e $\mathcal{P} . \mathcal{I} \subseteq(G R A)_{0}$. O funtor $\mathcal{E}_{\mathcal{D P} \mathcal{I}}: c-$ $(\mathcal{P}: \mathcal{I})-E S Q \rightarrow g-(\mathcal{D}, \mathcal{P} ; \mathcal{I})-E S Q\left((S, c P, c I), F ;\left(S^{\prime}, c P^{\prime}, c I^{\prime}\right)\right) \mapsto((\mathcal{E}(S),\{d: \exists G \in$ 
$\mathcal{D}(d: G \rightarrow \mathcal{E}(S) \wedge(d . S)$ é diagrama comutativo em $S)\},\{p:(p . S) \in c P\}:\{i:(i . S) \in$ $c I\}): F:\left(\mathcal{E}\left(S^{\prime}\right),\left\{d^{\prime}: \exists G \in \mathcal{D}\left(d^{\prime}: \rightarrow \mathcal{E}\left(S^{\prime}\right) \wedge\left(d^{\prime}, S^{\prime}\right)\right.\right.\right.$ é diagrama comutativo em $\left.\left.S^{\prime}\right)\right\} ;\left\{p^{\prime}\left(p^{\prime} ; S^{\prime}\right) \in\right.$ $\left.\left.c P^{\prime}\right\}:\left\{i^{\prime}:\left(i^{\prime}: S^{\prime}\right) \in c I^{\prime}\right\}\right)$ (que está bem definido nos objetos e flechas !) tem adjunto à esquerda $\mathcal{G}_{\mathcal{D P} \mathcal{I}}$ com unidade $\mu$ tais que :

(i) $\forall \mathcal{S}=(S, D, c P, c I) \in \operatorname{obj}(g-(\mathcal{D}, \mathcal{P}, \mathcal{I})-E S Q)\left(\mathcal{E}\left(\mathcal{G}_{\mathcal{D P} \mathcal{I}}(\mathcal{S})=(\mathcal{S}: \mathcal{D}) \wedge \mathcal{E}\left(\mu_{\mathcal{S}}\right)=\right.\right.$ $\left.\nu(S, D): S \rightarrow \mathcal{E}((S: D)) \wedge \mathcal{E}_{\mathcal{D P} \mathcal{I})} \circ \mathcal{G}_{\mathcal{D P} \mathcal{I}}(\mathcal{S})=(\nu(\mathcal{S}, \mathcal{D}))_{*}(\mathcal{S})\right)$.

(ii) $\left.\forall U \in U N I V\left(\mathcal{S}=(S, D, c P, c I) \in U \Longleftrightarrow \mathcal{G}_{\mathcal{D P I}}(S) \in \mathcal{U}\right)\right)$.

(iii) $\forall U \in U N I V\left(\mathcal{D} \in \mathcal{P}\left((U-G R A)_{0}\right) \wedge \mathcal{P}: \mathcal{I} \subseteq(U-G R A)_{0} \Rightarrow\left(\left(\mathcal{G}_{\mathcal{D P} \mathcal{I} \mid ;} \mathcal{E}_{\mathcal{D P} \mathcal{I} \mid)}\right.\right.\right.$ : $g-(\mathcal{D}, \mathcal{P}, \mathcal{I})-U-E S Q \rightarrow c-(\mathcal{P}, \mathcal{I})-U-E S Q$ é adjunção $))$.

(iv) Se o "grafo quadrado" $\cong G_{2}^{2}$ (ver observação 3 abaixo) é elemento de $\mathcal{D}$ então temos estrutura natural de categoria sobre classe (de objetos) $g-(\mathcal{D}, \mathcal{P}, \mathcal{I})-E S Q\left(\mathcal{S}, \mathcal{E}_{\mathcal{D P} \mathcal{I}}(\overline{\mathcal{S}})\right)$ e obtemos isomorfismo de categorias $c-(\mathcal{P}, \mathcal{I})-E S Q\left(\mathcal{G}_{\mathcal{D P} \mathcal{I}}(\mathcal{S}), \overline{\mathcal{S}}\right) \cong g-(\mathcal{D}, \mathcal{P} ; \mathcal{I})-$ $\operatorname{ESQ}\left(\mathcal{S}, \mathcal{E}_{\mathcal{D P} \mathcal{I}}(\overline{\mathcal{S}})\right)$ natural em $\mathcal{S} \in \operatorname{obj}\left((g-(\mathcal{D}, \mathcal{P}, \mathcal{I})-E S Q)^{\star}\right)$ e $\overline{\mathcal{S}} \in \operatorname{obj}(c-(\mathcal{P}, \mathcal{I})-E S Q)$

Proposição 2: Sejam $\mathcal{P}: \mathcal{I} \subseteq(G R A)_{0}$. O funtor de inclusão $I_{\mathcal{P} \mathcal{I}}: c c-(\mathcal{P}, \mathcal{I})-E S Q \hookrightarrow$ $c-(\mathcal{P}, \mathcal{I})-E S Q$ tem adjunto à esquerda $L_{\mathcal{P} \mathcal{I}}$ com unidade $\mu$ tais que :

(i) $\forall \mathcal{S} \in \operatorname{obj}(c-(\mathcal{P}, \mathcal{I})-E S Q)\left(\mathcal{E}\left(L_{\mathcal{P} \mathcal{I}}(\mathcal{S})=S /\left\langle R(c P, c I)>\wedge \mathcal{E}\left(\mu_{\mathcal{S}}\right)=\Pi_{\langle R(c P, c I)\rangle}\right.\right.\right.$ : $\left.S \rightarrow \mathcal{E}(S /<R(c P, c I)>) \wedge I_{\mathcal{P I}} \circ L_{\mathcal{P I}}(\mathcal{S})=\left(\Pi_{\langle R(c P, c I)>}\right)_{*}(\mathcal{S})\right)$ onde $\forall s, t \subseteq S_{0} R(c P, c I)(s, t)=$ $\left\{\left(f, f^{\prime}\right) \in S(s, t) \times S(s, t): \exists p: G_{-} \rightarrow \mathcal{E}(S) \in c P \exists g \stackrel{h}{\leftarrow} g^{\prime} \in G\left(s=p(-\infty) \wedge f=p\left(\pi_{g}\right) \wedge f^{\prime}=\right.\right.$ $\left.\left.p(h) \circ p\left(\pi_{g^{\prime}}\right)\right)\right\} \cup\left\{\left(f: f^{\prime}\right) \in S(s, t) \times S(s, t): \exists i: G_{+} \rightarrow \mathcal{E}(S) \in c I \exists g \stackrel{h}{\rightarrow} g^{\prime} \in G(t=i(+\infty) \wedge f=\right.$ $\left.\left.i\left(\iota_{g}\right) \wedge f^{\prime}=i\left(\iota_{g^{\prime}}\right) \circ i(h)\right)\right\}$.

(ii) $\left.\forall U \in U N I V\left(\mathcal{S} \in U \Longleftrightarrow L_{\mathcal{P}, I}(\mathcal{S}) \in U\right)\right)$.

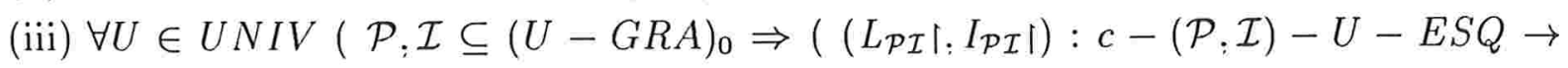
$c c-(\mathcal{P}, \mathcal{I})-U-E S Q$ é adjunção ) ) .

(iv) Temos isomorfismo de categorias $c c-(\mathcal{P} ; \mathcal{I})-E S Q\left(L_{\mathcal{P} \mathcal{I}}(\mathcal{S}), \overline{\mathcal{S}}\right) \cong c-(\mathcal{P} ; \mathcal{I})-$ $\operatorname{ESQ}\left(\mathcal{S}, I_{\mathcal{P} \mathcal{I}}(\overline{\mathcal{S}})\right)$ natural em $\mathcal{S} \in \operatorname{obj}\left((c-(\mathcal{P}, \mathcal{I})-E S Q)^{\star}\right)$ e $\overline{\mathcal{S}} \in \operatorname{obj}(c c-(\mathcal{P}, \mathcal{I})-E S Q)$.

\section{Observação 3 :}

(i) Se $\mathcal{S}$ é um g-esboço tal que $\mathcal{D}_{\mathcal{S} 1}\left[\left\{G \in(G R A)_{0}: \# G<\omega\right\}\right] \leq \omega$ então $\tau(\mathcal{S})=$ máx $\{\#$ S: \#P:\#I, $\pi(\mathcal{S}), \iota(\mathcal{S})\}$.

(ii) Para cada $0 \leq m \leq n<\omega$ consideramos os grafos, únicos a menos de isomorfismos, $\vec{n} ; \vec{m}$ :

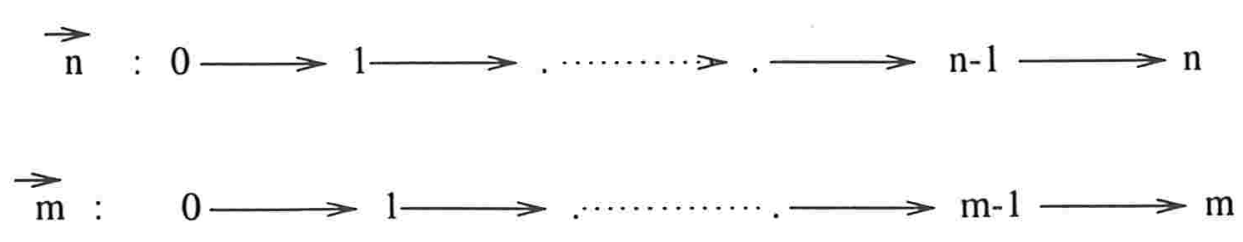


Consideramos $G_{m}^{n}$ o grafo .único a menos de isomorfismos, obtido através da "identificação dos extremidades" de $\vec{n}$ e $\vec{m}$ :

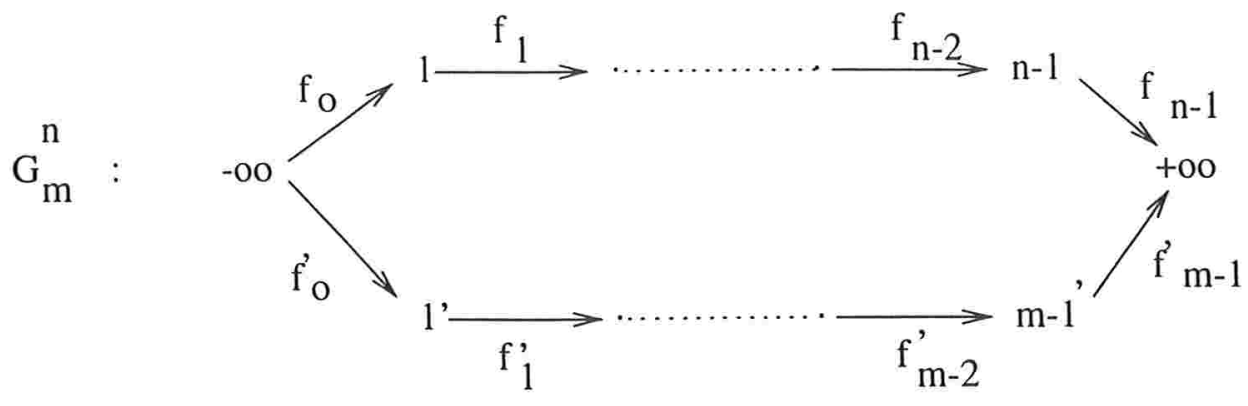

Fixemos construções tais que para todo universo $U: \mathcal{D}=\left\{G_{m}^{n}: 0 \leq m \leq n<\omega\right\} \subseteq$ ${ }_{o b j}(U-G R A)$. Denominamos esboços (gráficos) naturais os g-esboços $\mathcal{S}$ tais que $\mathcal{D}_{\mathcal{S}} \subseteq \mathcal{D}$ - A proposição 1 nos permite intercambiar "localmente" gn-esboços e c-esboços ; assim utilizaremos $E S Q$ para designar ambas $g n-E S Q$ e $c-E S Q$.

As três proposições abaixo estão demonstradas, com pequenas alterações em [029] .

Proposição $3:$ Sejam $\mathcal{P}, \mathcal{I} \subseteq(G R A)_{0}$. O funtor de inclusão $I_{\mathcal{P} \mathcal{I}}: c l-(\mathcal{P} ; \mathcal{I})-E S Q \hookrightarrow$ $c c-(\mathcal{P}, \mathcal{I})-E S Q$ tem adjunto à esquerda $L_{\mathcal{P} \mathcal{I}}$ com unidade $\mu$ tais que :

(i) $\forall \mathcal{S} \in \operatorname{obj}(c c-(\mathcal{P}, \mathcal{I})-E S Q)$ denotando $\mathcal{E}\left(L_{\mathcal{P} \mathcal{I}}(\mathcal{S})=S_{l}\right.$ e $\mathcal{E}\left(\mu_{\mathcal{S}}\right)=\sigma_{S}: S \rightarrow \mathcal{E}(S /<$ $R(c P, c I)>$ ) então $L_{\mathcal{P} \mathcal{I}}(\mathcal{S})$ é tal que $\left.I_{\mathcal{P} \mathcal{I}} \circ L_{\mathcal{P} \mathcal{I}}(\mathcal{S})=\left(\sigma_{S}\right)_{*}(S)\right)$.

(ii) $\left.\forall U \in U N I V\left(\mathcal{S} \in U \Longleftrightarrow L_{\mathcal{P} \mathcal{I}}(\mathcal{S}) \in U\right)\right)$.

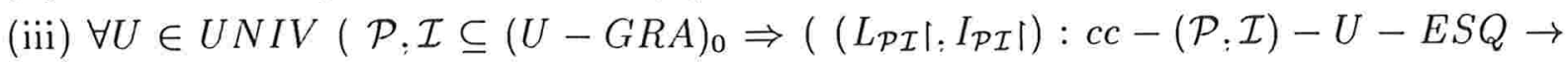
$c l-(\mathcal{P}: \mathcal{I})-U-E S Q$ é adjunção ) ) .

(iv) Temos isomorfismo de categorias $c l-(\mathcal{P}, \mathcal{I})-E S Q\left(L_{\mathcal{P} I}(\mathcal{S}), \overline{\mathcal{S}}\right) \cong c c-(\mathcal{P}, \mathcal{I})-$ $\operatorname{ESQ}\left(\mathcal{S}, I_{\mathcal{P} \mathcal{I}}(\overline{\mathcal{S}})\right)$ natural em $\mathcal{S} \in o b j\left((c c-(\mathcal{P}, \mathcal{I})-E S Q)^{\star}\right)$ e $\overline{\mathcal{S}} \in o b j(c l-(\mathcal{P}, \mathcal{I})-E S Q)$.

Proposição $4:$ Sejam $\mathcal{P}: \mathcal{I} \in \mathcal{P}\left((G R A)_{0}\right)$. O funtor $(\mathcal{P}, \mathcal{I})-T E O \stackrel{R}{\rightarrow} c l-(\mathcal{P}, \mathcal{I})-E S Q$ $=(\mathcal{P}, \mathcal{I})-T E O \stackrel{(\text { incl) }}{\longrightarrow} c l-E S Q \stackrel{\text { rest }}{\rightarrow} c l-(\mathcal{P}, \mathcal{I})-E S Q$ é tal que $\forall S \in o b j(c l-(\mathcal{P} ; \mathcal{I})-E S Q)$ $\exists \mathcal{S}^{\prime} \in \operatorname{obj}((\mathcal{P}, \mathcal{I})-T E O) \exists J_{\mathcal{S}}: \mathcal{S} \rightarrow R_{\mathcal{P} \mathcal{I}}\left(\mathcal{S}^{\prime}\right)$ satisfazendo:

(i) $\forall \bar{S} \in \operatorname{obj}((\mathcal{P}, \mathcal{I})-T E O)\left[J_{\overline{\mathcal{S}}}\right]:(\mathcal{P}, \mathcal{I})-T E O\left(\mathcal{S}^{\prime}, \overline{\mathcal{S}}\right) \rightarrow c l-(\mathcal{P}, \mathcal{I})-E S Q\left(\mathcal{S}, R_{\mathcal{P I}}(\overline{\mathcal{S}})\right)$ é hom-bijetor e sobrejetor nos objetos (logo é um funtor de equivalência).

(ii) $\forall U \in U N I V\left(\mathcal{P}, \mathcal{I} \in \mathcal{P}\left((U-G R A)_{0}\right) \Rightarrow\left(\mathcal{S} \in U \Longleftrightarrow \mathcal{S}^{\prime} \in U\right)\right.$ (logo a propriedade acima se "restringe aos universos").

Proposição $5:$ Sejam $\mathcal{P}: \mathcal{I} \in \mathcal{P}\left((G R A)_{0}\right)$. O funtor $(\mathcal{P}, \mathcal{I})-T Y P \stackrel{R}{\rightarrow} c l-(\mathcal{P}, \mathcal{I})-E S Q$ $=(\mathcal{P}, \mathcal{I})-T Y P \stackrel{(i n c l)}{\longrightarrow} c l-E S Q \stackrel{\text { rest }}{\rightarrow} c l-(\mathcal{P}, \mathcal{I})-E S Q$ tem adjunto à esquerda $L_{\mathcal{P} I}$ com unidade $\mu$ tais que : 
(i) $\forall U \in U N I V\left(\mathcal{P}, \mathcal{I} \in \mathcal{P}\left((U-G R A)_{0}\right) \Rightarrow\left(\left(L_{\mathcal{P} \mathcal{I} \mid .} R_{\mathcal{P} \mathcal{I} \mid}\right): c l-(\mathcal{P} . \mathcal{I})-U-E S Q \rightarrow\right.\right.$ $(\mathcal{P}, \mathcal{I})-U-T Y P$ é adjunção ) ) .

(ii) Temos isomorfismo de categorias $(\mathcal{P}, \mathcal{I})-T Y P\left(L_{\mathcal{P} \mathcal{I}}(\mathcal{S}), \overline{\mathcal{S}}\right) \cong c l-(\mathcal{P}, \mathcal{I})-E S Q\left(\mathcal{S}, R_{\mathcal{P}}(\overline{\mathcal{S}})\right)$ natural em $\mathcal{S} \in \operatorname{obj}\left((c l-(\mathcal{P} . \mathcal{I})-E S Q)^{\star}\right)$ e $\overrightarrow{\mathcal{S}} \in \operatorname{obj}((\mathcal{P} . \mathcal{I})-T Y P)$.

Fixamos três universos $U_{0} \in U_{1} \in U_{2} \in V$ com cardinais $\theta_{0}<\theta_{1}<\theta_{2}<\infty$

Observação 4 :

(i) Nos deparamos com as propriedades "2-universais" das teorias no ítem (1.3.3) através da noção de $\mathcal{I}$-completamento indutivo fraco. Por [127] e as proposições 2 e 1 temos que para $\mathcal{P}, \mathcal{I} \in\left[\operatorname{obj}\left(U_{0}-G R A\right)\right]^{<\theta_{0}}$ e todo $\mathcal{S}, U_{1}$-esboço $\mathcal{I}$-indutivo $(\mathcal{P}$-projetivo) essencialmente em $U_{0}$ admite uma $U_{1}-(\emptyset, \mathcal{I})$-teoria $\mathcal{S}^{\prime}\left(U_{1}-(\mathcal{P} ; \emptyset)\right.$-teoria $\left.\mathcal{S}^{\prime}\right)$ essencialmente em $U_{0}$ e um morfismo $J_{\mathcal{S}}: \mathcal{S} \rightarrow \mathcal{S}^{\prime}$ tal que $\forall \bar{S} \in \operatorname{obj}((\mathcal{P}, \mathcal{I})-T E O)\left[J_{\overline{\mathcal{S}}}\right]:(\mathcal{P}: \mathcal{I})-T E O\left(\mathcal{S}^{\prime}, \overline{\mathcal{S}}\right)$ $\rightarrow c l-(\mathcal{P} ; \mathcal{I})-\operatorname{ESQ}\left(\mathcal{S}, R_{\mathcal{P}}(\overline{\mathcal{S}})\right)$ é um funtor de equivalência com quasi-inversa o funtor extensão de Kan pontual à esquerda (à direita).

(ii) Sejam $\bar{S} \in \operatorname{obj}(C A T)$ e $\mathcal{T}_{U_{0}}(\bar{S})=\overline{\mathcal{S}}$ a " $U_{0}$-teoria máxima em $\bar{S}$ ": i.e. $\bar{S}$ munida de todos os seus cones projetivos/indutivos limites $U_{0}$-indexados. Sejam $\mathcal{P} \in[\mathcal{P}(\overline{\mathcal{S}})]^{<\theta_{0}}$ e $\mathcal{I} \in[\mathcal{I}(\overline{\mathcal{S}})]^{<\theta_{0}}$ então para todo esboço natural $\mathcal{S}$ tal que $\mathcal{P}(\mathcal{S}) \subseteq \mathcal{P}$ e $\mathcal{I}(\mathcal{S}) \subseteq \mathcal{I}$ temos pelas proposições $1,2,3,5,4$ temos $g n-E S Q\left(\mathcal{S}, \mathcal{E}^{(1)}(\overline{\mathcal{S}})\right) \cong c-E S Q\left(\left(\mathcal{S}^{\prime}\right)^{(1)}, \overline{\mathcal{S}}\right) \cong$

$$
c c-E S Q\left(\left(\mathcal{S}^{\prime}\right)^{(2,1)}, \overline{\mathcal{S}}\right) \cong c l-E S Q\left((\mathcal{S})^{(3,2,1)}, \overline{\mathcal{S}}\right) \cong(\mathcal{P}, \mathcal{I})-T Y P\left((\mathcal{S})^{(5,3,2,1)}, \overline{\mathcal{S}}\right) \simeq(\mathcal{P}, \mathcal{I})-
$$
$T E O\left((\mathcal{S})^{(4,5,3,2,1)}, \overline{\mathcal{S}}\right)$.

\section{Definição 5 :}

(i) Para cada $B \in \operatorname{obj}\left(U_{1}-C A T\right)$ consideramos o 2-funtor (" $B$-categoria de modelos") : $\operatorname{MOD}\left({ }_{-}, B\right)=c-\mathcal{E S Q}\left({ }_{-}, \mathcal{T}_{U_{0}}(B)\right):\left(c-U_{1}-E S Q\right)^{\star} \rightarrow U_{1}-\mathcal{C} \mathcal{A} \mathcal{T}$.

(ii) $A$ é $U_{1}$-categoria esboçável $\Longleftrightarrow \exists \mathcal{S} U_{1}$-esboço essencialmente em $U_{0} \exists A \stackrel{\cong}{\rightleftarrows} M O D\left(\mathcal{S}, U_{0}-\right.$ $S E T)$.

(iii) $F \in U_{1}-C A T\left(A, A^{\prime}\right)$ é esboçável $\Longleftrightarrow \exists \mathcal{S}^{\prime} \stackrel{\mathcal{H}}{\rightarrow} \mathcal{S}$ diagrama de $U_{1}$-esboços essencialmente em $U_{0}$ tal que existe diagrama como abaixo que comuta a menos de isomorfismo natural :

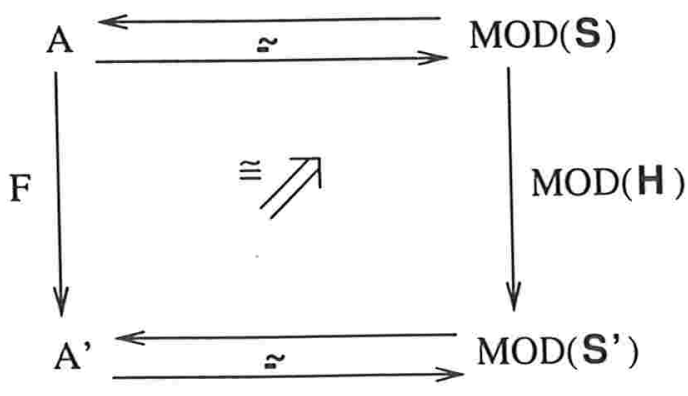

\section{Observação 5 :}

(i) Nas definições 5.(ii) e 5.(iii) podemos nos restringir que a $U_{0^{-}}$esboços . 
(ii) A simplicidade da noçào de morfismo de esboços torna esta categoria um pouco "estreita" : dois esboços com bases de cones isomorfas eventualmente não admitem morfismos entre eles! A situação é bem diferente quando consideramos componentes conexas, de fato. $U_{0}-E S Q$ é $o b j\left(U_{0}-C A T\right)$-filtrada. Deste modo podemos mostrar que se $B$ é $U_{0}$-ind completa então todo $U_{0}$-esboço possui categoria de $B$-modelos equivalente à categoria de $C$-modelos de um $U_{0}$-esboço $\theta_{0}$-geométrico com mesmos cardinais característicos.

Proposição 6 : Sejam $A \in \operatorname{obj}(C A T), \mathcal{S} \in \operatorname{obj}\left(c-U_{0}-E S Q\right)$ e $M O D(\mathcal{S}, A) \stackrel{J_{\mathcal{S}}}{\longmapsto}[S, A]$ $=\operatorname{MOD}(\mathcal{S}, A) \stackrel{M O D(i, A)}{\rightarrow} M O D\left(\mathcal{S}_{T}, A\right) \stackrel{\cong}{\rightrightarrows}[S ; A]$ onde $(S, \emptyset, \emptyset)=\mathcal{S}_{T} \stackrel{i}{\hookrightarrow} \mathcal{S}=(S ; c P, c I)$ e $A$ é $\mathcal{P}_{\mathcal{S}}$-proj completa e $\mathcal{I}_{\mathcal{S}}$-ind completa. Temos:

(i) $J_{\mathcal{S}}$ cria $I S O\left(A, \mathcal{P}_{\mathcal{S}}\right)$-limites indutivos.

(ii) $J_{\mathcal{S}}$ cria $\underset{I S O}{\stackrel{S}{\leftrightarrows}}\left(A, \mathcal{I}_{\mathcal{S}}\right)$-limites projetivos.

(i) Sejam $X \in \underset{\longrightarrow}{\operatorname{SO}}\left(A, \mathcal{P}_{\mathcal{S}}\right)$ e $\left(X \stackrel{M}{\rightarrow} A^{S}\right)=\left(X \stackrel{\mathcal{M}}{\rightarrow} M O D(\mathcal{S}, A) \stackrel{J_{\mathcal{S}}}{\rightarrow} A^{S}\right)$. Mostraremos

que $\forall G^{+} \stackrel{i}{\rightarrow} S\left(G^{+} \stackrel{(\lim M(x)) \text { oi }}{x} \longrightarrow A \in(\operatorname{contindlim}(G, A))_{0}\right)$ e $\forall G^{-} \stackrel{p}{\rightarrow} S\left(G^{-} \stackrel{(\lim M(x)) \text { op }}{\longrightarrow} \longrightarrow A \in\right.$ (coneprojlim $\left.(G, A))_{0}\right)$.

. $\mathcal{S}=\emptyset \Rightarrow \mathbf{1} \cong \operatorname{MOD}(\mathcal{S}, A) \stackrel{J_{S}}{\mapsto}[S, A] \cong \mathbf{1} \mathrm{ok}$.

$\mathcal{S} \neq \emptyset$ :

.. Como $A$ é $X$-ind completa para todo $i: G^{+} \rightarrow S \in c I$ temos que o funtor $\left(A^{S} \stackrel{\hat{i}}{\rightarrow} A^{\left(G^{+}\right)}\right)$ $=\left(A^{S} \stackrel{A^{\epsilon} S}{\rightarrow} A^{\mathcal{L}(\mathcal{E}(S))} \stackrel{\cong}{\rightarrow} A^{\mathcal{E}(S)} \stackrel{A^{i}}{\rightarrow} A^{\left(G^{+}\right)}\right)$

preserva $X$-limites indutivos, logo $\underset{X}{\lim } \hat{i}(M(x)) \stackrel{\cong}{\rightarrow}$

$\underset{X}{(\lim } M(x)) \circ i$; pela definição de $M$ temos que $\left(X \stackrel{i \circ M}{\rightarrow} A^{\left(G^{+}\right)}\right)=(X \stackrel{\Gamma}{\rightarrow} \operatorname{con} \operatorname{ind} \operatorname{dim}(G, A) \hookrightarrow$ $A^{\left(G^{+}\right)}$) e o resultado segue pelas observações 1.(ii) e 2 do ítem (1.3.2) .

.. Como $A$ é $X$-ind completa para todo $p: G^{-} \rightarrow S \in c P$ temos que o funtor $\left(A^{S} \stackrel{\hat{p}}{\rightarrow} A^{\left(G^{-}\right)}\right)=\left(A^{S} \stackrel{A^{\epsilon} S}{\rightarrow} A^{\mathcal{L}(\mathcal{E}(S))} \stackrel{\cong}{\rightarrow} A^{\mathcal{E}(S)} \stackrel{A^{p}}{\rightarrow} A^{\left(G^{-}\right)}\right)$preserva $X$-limites indutivos, logo $\underset{X}{\lim } \bar{p}(M(x)) \stackrel{\cong}{\rightarrow}$

$\underset{X}{(\lim } M(x)) \circ p$; pela definição de $M$ temos que $\left(X \stackrel{\hat{p} \circ M}{\rightarrow} A^{\left(G^{-}\right)}\right)=(X \stackrel{\Gamma}{\rightarrow} \operatorname{con} \epsilon \operatorname{proj} \lim (G, A) \hookrightarrow$ $\left.A^{\left(G^{-}\right)}\right)$e o resultado segue pela escolha de $X$ e pelas observações 1.(iii) e 2 do ítem (1.3.2) .

(ii) Sejam $X \in \underset{\longleftarrow}{\longleftarrow}\left(A, \mathcal{I}_{\mathcal{S}}\right)$ e $\left(X \stackrel{M}{\rightarrow} A^{S}\right)=\left(X \stackrel{\mathcal{M}}{\rightarrow} M O D(\mathcal{S}, A) \stackrel{J_{\mathcal{S}}}{\rightarrow} A^{S}\right)$. Mostraremos $\stackrel{(\lim M(x)) \mathrm{op}}{\longleftarrow}(\lim M(x)) \circ i$ que $\forall G^{-} \stackrel{p}{\rightarrow} S\left(G^{-} \stackrel{\stackrel{x}{x}}{\longrightarrow} \quad A \in(\operatorname{contprojlim}(G, A))_{0}\right)$ e $\forall G^{+} \stackrel{i}{\rightarrow} S\left(G^{+} \stackrel{\stackrel{x}{x}}{\longrightarrow} A \in\right.$ (contindlim $\left.(G, A))_{0}\right)$.

. $S=\emptyset \Rightarrow 1 \cong \operatorname{MOD}(\mathcal{S}, A) \stackrel{J_{S}}{\sqcup}[S, A] \cong 1 \mathrm{ok}$.

$\mathcal{S} \neq \emptyset$ :

.. Como $A$ é $X$-proj completa para todo $p: G^{-} \rightarrow S \in c P$ temos que o funtor 
$\left(A^{S} \stackrel{\hat{p}}{\rightarrow} A^{\left(G^{-}\right)}\right)=\left(A^{S} \stackrel{A^{\epsilon} S}{\rightarrow} A^{\mathcal{L}(\mathcal{E}(S))} \stackrel{\cong}{\rightrightarrows} A^{\mathcal{E}(S)} \stackrel{A^{p}}{\rightarrow} A^{\left(G^{-}\right)}\right)$preserva $X^{\prime}$-limites projetivos; logo $\underset{x}{\lim } \hat{p}(M(x)) \stackrel{\Xi}{\rightrightarrows}$

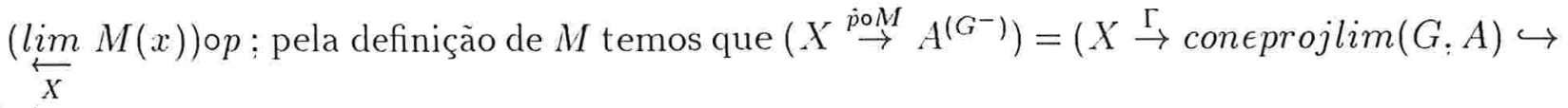
$\left.A^{\left(G^{-}\right)}\right)$e o resultado segue pelas observações 1.(i) e 2 do ítem (1.3.2) .

.. Como $A$ é $X$-proj completa para todo $i: G^{+} \rightarrow S \in c I$ temos que o funtor $\left(A^{S} \stackrel{\hat{i}}{\rightarrow} A^{\left(G^{+}\right)}\right)=\left(A^{S} \stackrel{A^{\epsilon} S}{\rightarrow} A^{\mathcal{L}(\mathcal{E}(S))} \stackrel{\cong}{\rightarrow} A^{\mathcal{E}(S)} \stackrel{A^{i}}{\rightarrow} A^{\left(G^{+}\right)}\right)$preserva $X$-limites projetivos, logo $\underset{X}{\lim } \bar{i}(M(x)) \stackrel{\cong}{\leftrightarrows} \underset{X}{\lim } M(x)) \circ i$; pela definição de $M$ temos que $\left(X \stackrel{\hat{i} \circ M}{\rightarrow} A^{\left(G^{+}\right)}\right)=$ $\left(X \stackrel{\Gamma}{\rightarrow} \operatorname{con} \epsilon i n d l i m(G, A) \hookrightarrow A^{\left(G^{+}\right)}\right)$e o resultado segue pela escolha de $X$ e pelas observações 1.(iii) e 2 do ítem (1.3.2) .

Proposição 7 : Sejam $\mathcal{S}: \mathcal{S}^{\prime}$ c-esboços . Para cada $s \in S_{0}$ e $s^{\prime} \in S_{0}^{\prime}$ consideramos os funtores de "inclusão " $: j_{s}: S^{\prime} \rightarrow S \times S^{\prime}$ e $j_{s^{\prime}}: S \rightarrow S \times S^{\prime}$. Denotando $\mathcal{S} \otimes \mathcal{S}^{\prime}$ o c-esboço com categoria subjacente $S \times S^{\prime}$ e estrutura $\left(\bigvee_{s \in S_{0}}\left(j_{s}\right)_{*}\left(\mathcal{S}^{\prime}\right)\right) \vee\left(\bigvee_{s^{\prime} \in S_{0}^{\prime}}\left(j_{s^{\prime}}\right)_{*}(\mathcal{S})\right)$ temos, para todo c-esboço $\mathcal{S}^{\prime \prime}$, mergulho evidente $c-E S Q\left(\mathcal{S} \otimes \mathcal{S}^{\prime}, \mathcal{S}^{\prime \prime}\right) \rightarrow C A T(S, c-$ $\left.E S Q\left(\mathcal{S}^{\prime} ; \mathcal{S}^{\prime \prime}\right)\right)$. Se $\left\{\mathcal{S}, \mathcal{S}^{\prime}\right\} \subseteq o b j\left(c-U_{0}-E S Q\right)$ e $A$ é uma categoria $\mathcal{P}(\mathcal{S}) \cup \mathcal{P}\left(\mathcal{S}^{\prime}\right)$-proj completa e $\mathcal{I}(\mathcal{S}) \cup \mathcal{I}\left(\mathcal{S}^{\prime}\right)$-ind completa tal que, em $A, \mathcal{I}(\mathcal{S})$-limites indutivos comutam com $\mathcal{P}\left(\mathcal{S}^{\prime}\right)$ - limites projetivos e $\mathcal{I}\left(\mathcal{S}^{\prime}\right)$-limites indutivos comutam com $\mathcal{P}(\mathcal{S})$-limites projetivos então temos $\operatorname{MOD}\left(\mathcal{S} \otimes \mathcal{S}^{\prime}, A\right) \stackrel{\widetilde{\rightrightarrows}}{M O D}\left(\mathcal{S}, M O D\left(\mathcal{S}^{\prime} ; A\right)\right)$.

Observação 6 : Se $\mathcal{S}_{\text {inic }}=(1, \emptyset,\{\emptyset+\stackrel{! \cong}{\rightarrow} 1\})$ e $\mathcal{S}_{\text {fin }}=(1,\{\emptyset-\stackrel{! \cong}{\rightrightarrows} 1\}, \emptyset)$ temos $\mathcal{S}_{\text {inic }} \otimes$ $\mathcal{S}_{\text {fin }} \cong \mathcal{S}_{\text {zero }}=(\mathbf{1},\{\emptyset-\stackrel{! \cong}{\rightarrow} 1\},\{\emptyset+\stackrel{\cong}{\rightarrow} 1\})$ e $\operatorname{MOD}\left(\mathcal{S}_{\text {inic }}, M O D\left(\mathcal{S}_{\text {fin }}, U_{0}-S E T\right)\right) \simeq \mathbf{1} \cong$ $\operatorname{MOD}\left(\mathcal{S}_{\text {fin }}, \operatorname{MOD}\left(\mathcal{S}_{\text {inic }}, U_{0}-S E T\right)\right) \operatorname{mas} \operatorname{MOD}\left(\mathcal{S}_{\text {zero }}, U_{0}-S E T\right)=\emptyset$.

A observação acima também revela que definir esboços ; mesmo muito simples, é tarefa pouco agradável.

Introduziremos abaixo uma "descrição gráfica" de um esboço natural $\mathcal{S}=(S, D, c P, c I)$ onde:

- Os símbolos $c^{*}: \downarrow$ : $\uparrow$ são utilizados para designar que os homomorfismos de grafos pertencem, respectivamente, a $D, c P, c I$.

. As notações empregadas são tais que para todos $A \in(C A T)_{0}$ e $\mathcal{M} \in(M O D(\mathcal{S} ; A))_{0}$. $\mathcal{M}$ "valida" os homomorfismos especificados.

(i) Identidade: " $f=1_{a}$ "

Um diagrama indexado por $G_{0}^{1}$ com imagem:
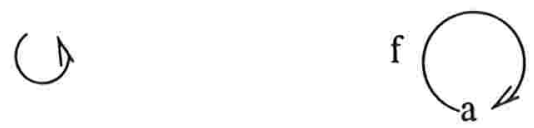
(ii) Isomorfismos inversos: " $g \circ f=1_{a} \wedge f \circ g=1_{b}$ " Dois diagramas indexados por $G_{0}^{2}$ :

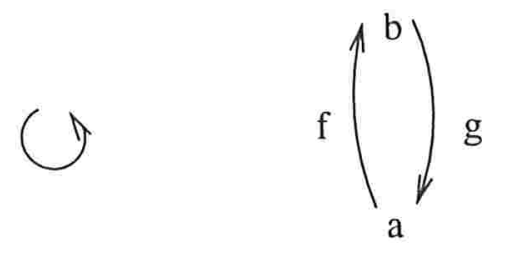

(iii) Isomorfismo : " $f: a \stackrel{\cong}{\rightarrow} b$ " Um cone projetivo com base indexada por grafo $\cong \mathbf{1}$ com imagem:

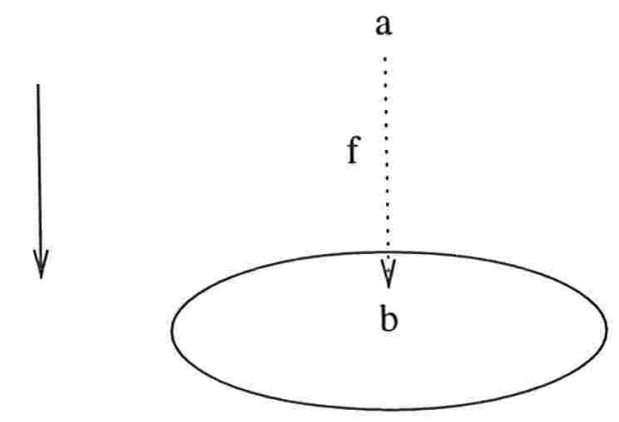

(iii)" Isomorfismo : " $f: a \stackrel{\cong}{\rightarrow} b$ " Um cone indutivo com base indexada por grafo $\cong \mathbf{1}$ com imagem:

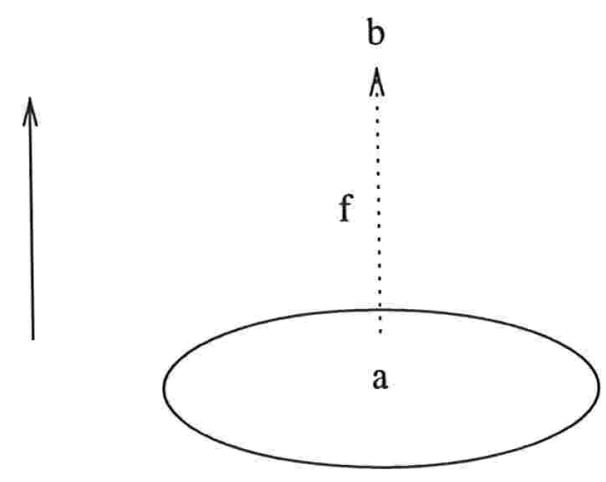

(iv) Monomorfismo : " $f: a \hookrightarrow b$ " Uma especificação de identidade e um cone projetivo com base indexada por grafo $\cong \rightarrow \leftarrow$ com imagem:

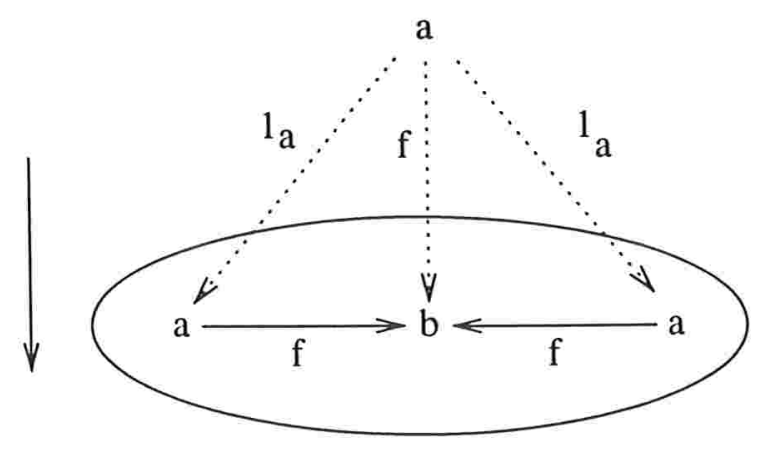


(iv)" Epimorfismo : " $f: a \rightarrow b$ "

Uma especificação de identidade e um cone indutivo com base indexada por grafo $\cong \leftarrow \rightarrow$ com imagem:

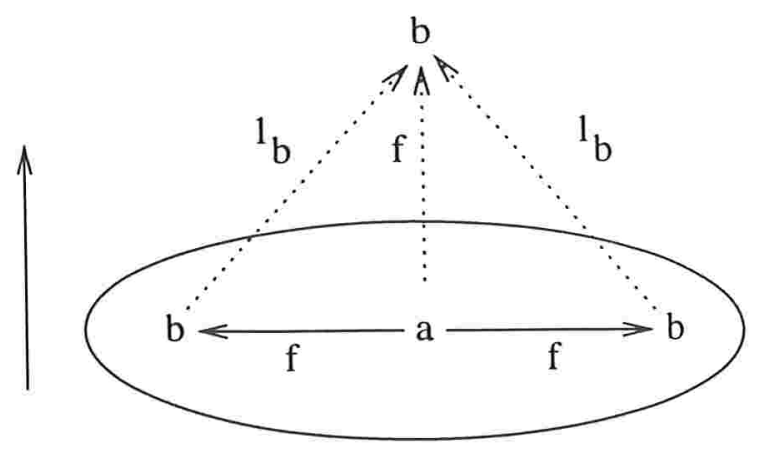

(v) Objeto final : " 1 "

Um cone projetivo com base indexada por $\emptyset$ com imagem:

1

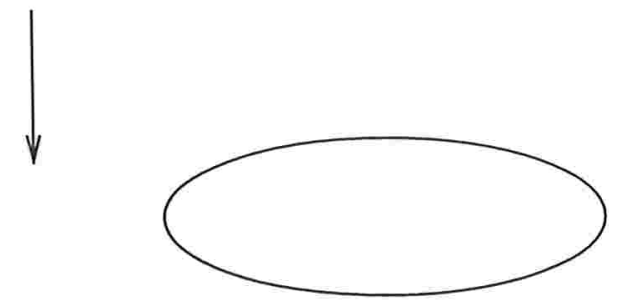

(v): Objeto inicial : "0"

Um cone indutivo com base indexada por $\emptyset$ com imagem:

0

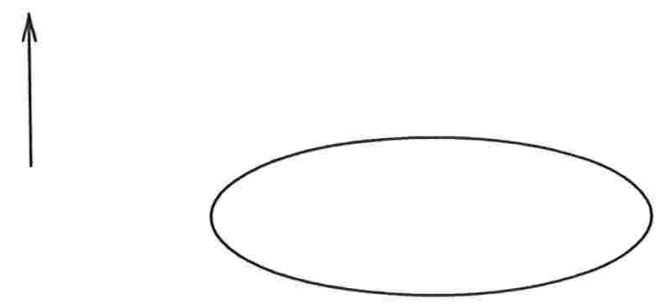

(vi) Produto: " $\prod_{i \in I} F(i)$ "

Um cone projetivo com base $F: I \rightarrow S$, indexado por grafo discreto $I$ com imagem: 


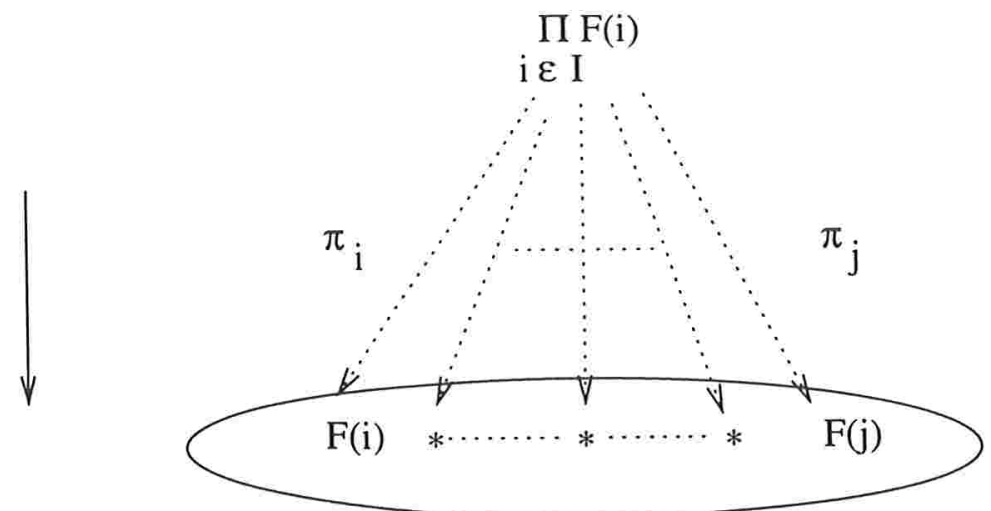

(vi)" Coproduto : " $\coprod_{i \in I} F(i)$ "

Um cone indutivo com base $F: I \rightarrow S$, indexado por grafo discreto $I$ com imagem:

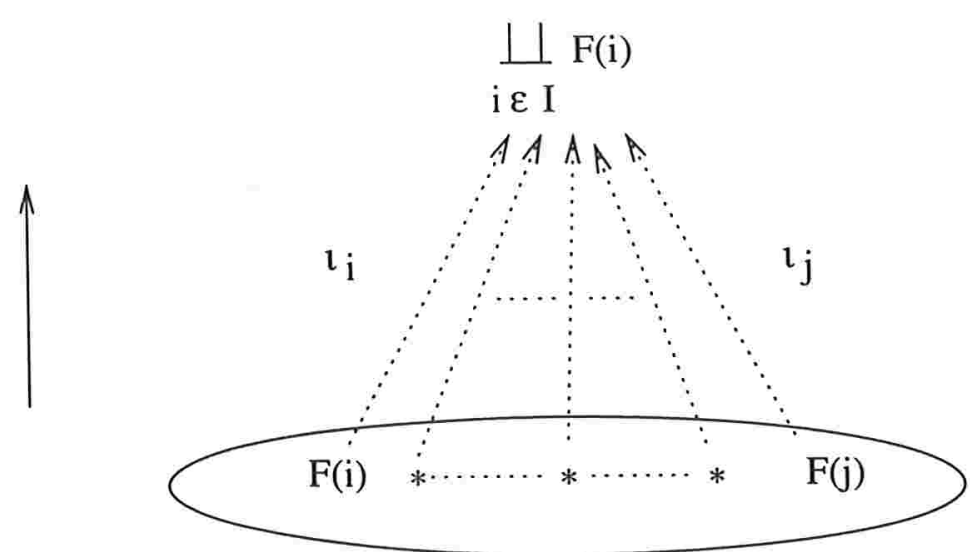

(vii) Igualador : "ig $\left(\left\{f_{i}\right\}_{i \in I}\right)$ "

Um cone projetivo com base indexada por grafo $\in \operatorname{Par}_{\alpha}(\alpha=\# I)$ com imagem:

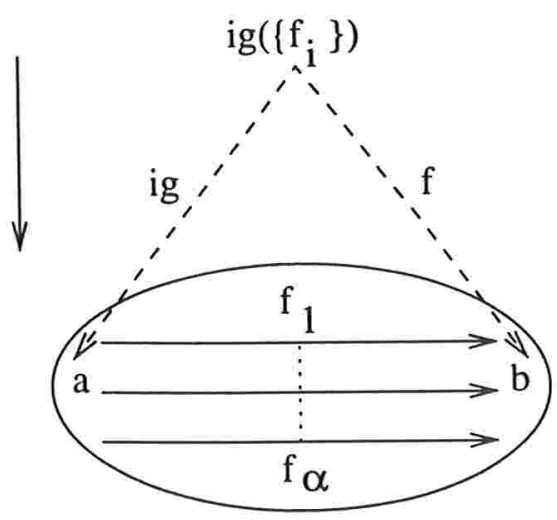

(vii): Coigualador : "coig $\left(\left\{f_{i}\right\}_{i \in I}\right)$ "

Um cone indutivo com base indexada por grafo $\in \operatorname{Par}_{\alpha}(\alpha=\# I)$ com imagem: 


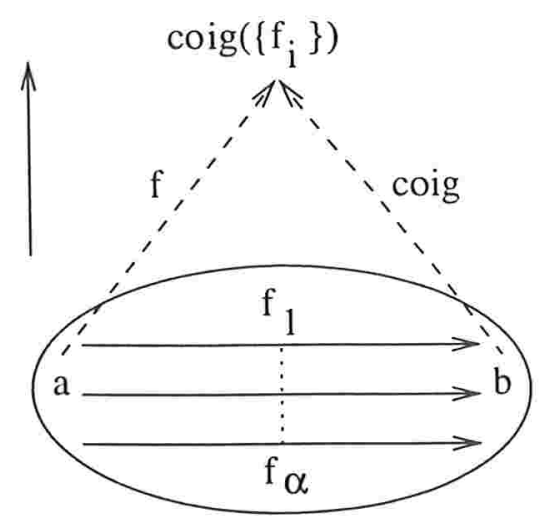

(viii)" Limite projetivo : " $\stackrel{\lim }{I}_{I} F(i)$ "

Um cone projetivo com base $F: I \rightarrow S:$

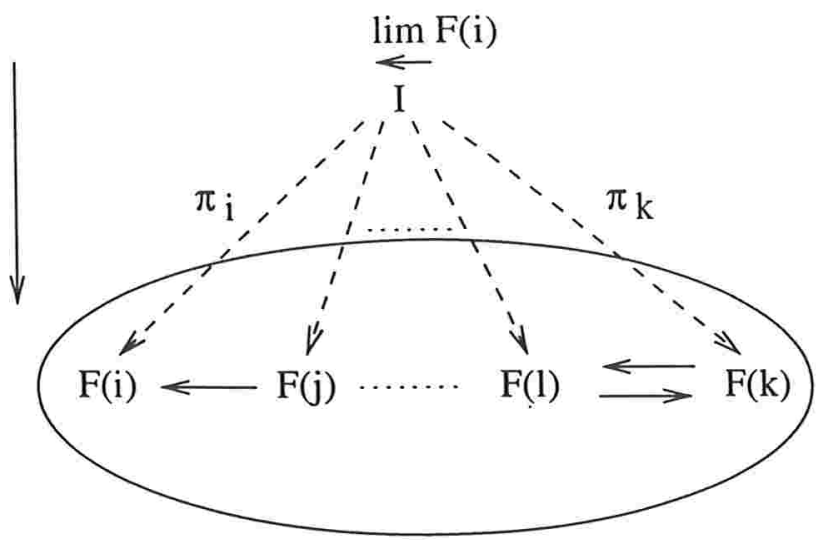

(viii)" Limite indutivo : $\underset{I}{\lim } F(i)$ "

Um cone indutivo com base $F: I \rightarrow S$ :

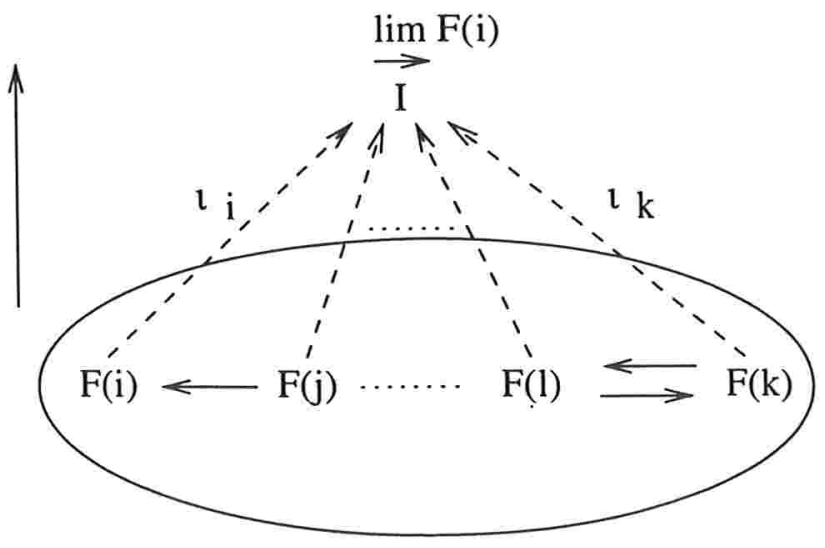

(ix) Flechas $\underset{\longleftarrow}{\lim }:$ " $\left(f_{i}\right)_{i \in I_{0}}: a \rightarrow \underset{I}{\lim } F(i)$ "

Um cone projetivo e \# $\left(I_{0}\right)$ diagramas indexados por $G_{1}^{2}$ com imagens: 


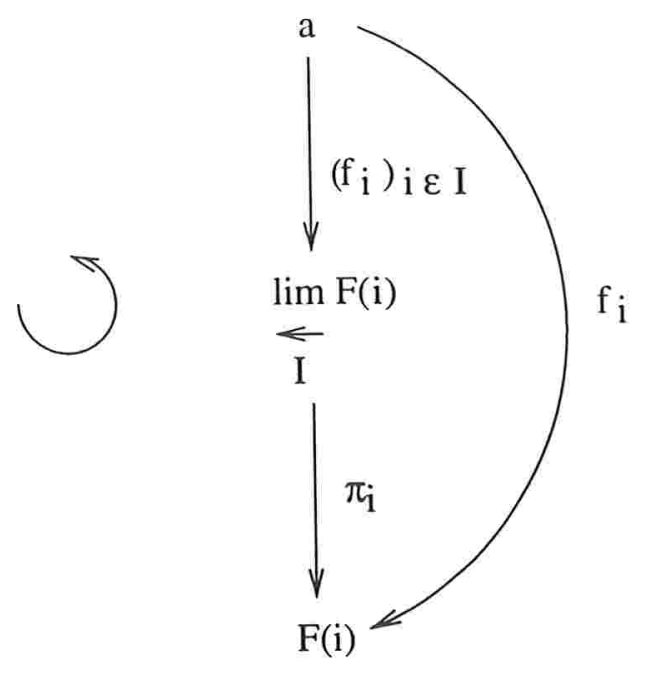

(ix)" Flechas $\underset{\longrightarrow}{\lim }:$ " $\left[f_{i}\right]_{i \in I_{0}}: \underset{I}{\lim } F(i) \rightarrow a$ " Um cone indutivo e \#(I0) diagramas indexados por $G_{1}^{2}$ com imagens:

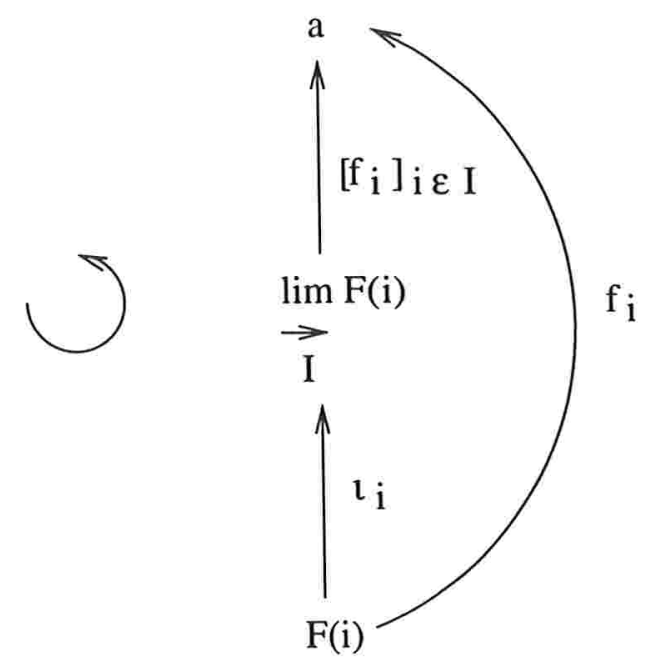

(x) $\underset{\longleftarrow}{\lim }$ de flechas : " $F^{\prime} \circ T \stackrel{\eta}{\rightarrow} F \Rightarrow \underset{I}{\lim } F^{\prime}(T(i)) \stackrel{\lim _{i} \eta_{i}}{\stackrel{I}{\rightarrow}} \underset{I}{\lim } F(i)$ "

Dois cones projetivos $I$-indexados e \# $\left(I_{0}\right)$ diagramas indexados por $G_{2}^{2}$ com imagens:

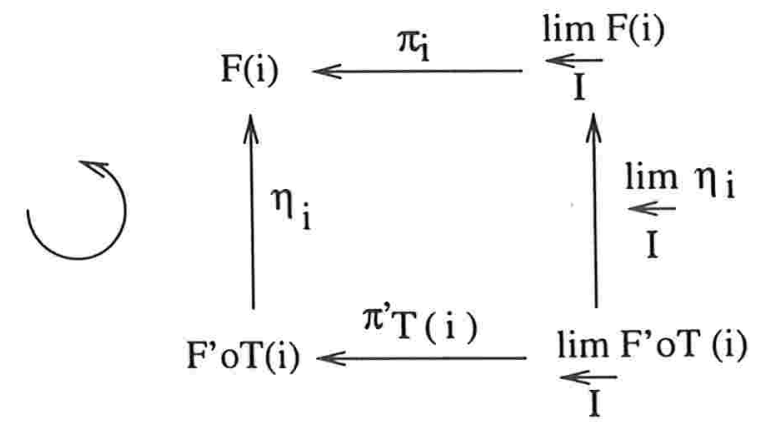


$(\mathrm{x})$ " $\stackrel{\lim }{\rightarrow}$ de flechas : " $F \stackrel{\eta_{1}}{\rightarrow} F^{\prime} \circ T \Rightarrow \underset{I}{\lim } F(i) \stackrel{\stackrel{\lim \eta_{1}}{\rightarrow}}{\rightarrow} \underset{I}{\rightarrow} \lim _{I} \circ F^{\prime}$

Dois cones indutivos $I$-indexados e \# $\left(I_{0}\right)$ diagramas indexados por $G_{2}^{2}$ com imagens:

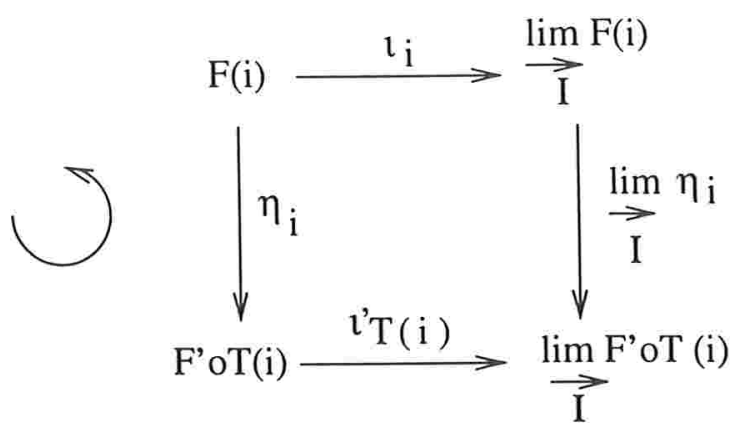




\section{Capítulo 4}

\section{Lógicas infinitárias}

Referências principais : [058] ; [168] : [166] .

\section{Definição 1 :}

(i)

- $\mu=\left(S, F, R, s_{F} ; a_{F} ; a_{R}\right)$ é um tipo (pequeno) $\Longleftrightarrow s_{F} \in S E T(F, S) ; \forall f \in F$ $\exists I_{f} \in C A R D\left(a_{F}(f) \in S E T\left(I_{f}, S\right)\right) ; \forall r \in R \exists I_{r} \in C A R D\left(a_{R}(r) \in S E T\left(I_{r}, S\right)\right)$.

. $s \in S$ é uma sorte de $\mu ; f \in F$ é uma símbolo funcional de $\mu$ com sorte de valor $s_{F}(f)$ e com aridade $a_{F}(f) ; r \in R$ é uma símbolo relacional de $\mu$ com aridade $a_{R}(r)$.

. $\pi(\mu)=\min \left\{\lambda: \omega \leq \lambda=r \epsilon g(\lambda)<\propto \wedge \forall f \in F\left(I_{f}<\lambda\right) \wedge \forall r \in R\left(I_{r}<\lambda\right)\right\}$ é o cardinal característico de $\mu$.

. $\tau(\mu)=\pi(\mu)+\# S+\# F+\# R$ é o cardinal de $\mu$.

(ii)

. Sejam $\mu=\left(S, F ; R, s_{F} ; a_{F} ; a_{R}\right)$ e $\mu^{\prime}=\left(S^{\prime} ; F^{\prime}, R^{\prime}, s_{F^{\prime}}, a_{F^{\prime}}, a_{R^{\prime}}\right)$ tipos .

$H=\left(\mu, H_{\sigma}, H_{\varphi}, H_{\rho}, \mu^{\prime}\right): \mu \rightarrow \mu^{\prime}$ é morfismo de tipos $\Longleftrightarrow H_{\sigma} \in S E T\left(S, S^{\prime}\right): H_{\varphi} \in$ $\operatorname{SET}\left(F, F^{\prime}\right) ; H_{\rho} \in \operatorname{SET}\left(R, R^{\prime}\right) ; s_{F^{\prime}} \circ H_{\varphi}=H_{\sigma} \circ s_{F} ; \forall f \in F\left(I_{H_{\varphi}(f)}=I_{f} \wedge a_{F^{\prime}}\left(H_{\varphi}(f)\right)=\right.$ $\left.H_{\sigma} \circ\left(a_{F}(f)\right)\right): \forall r \in R\left(I_{H_{\rho}(f)}=I_{r} \wedge a_{R^{\prime}}\left(H_{\rho}(r)\right)=H_{\sigma} \circ\left(a_{R}(r)\right)\right)$.

. Typ é a categoria dos tipos e morfismos de tipos .

. Seja $U \in U N I V$ :

.. $U-T y p$ é a subcategoria plena de Typ com objetos os tipos pertententes a $U$.

. $\mu \in o b j(T y p)$ é um tipo praticamente em $U \Longleftrightarrow \exists \mu^{\prime} \in o b j(U-T y p)\left(\mu \cong \mu^{\prime}\right)$.

. Seja $\mu=\left(S, F, R, s_{F} ; a_{F} ; a_{R}\right)$ tipo (tipo em $U$; tipo praticamente em $U$ ) :

.. $\left(S, F, \emptyset, s_{F}, a_{F}, \emptyset\right)=\mu_{A} \stackrel{i_{A}}{\longrightarrow} \mu$ é o tipo algébrico (em $U$; praticamente em $U$ ) associado a $\mu$.

.. $(S, \emptyset, \emptyset, \emptyset, \emptyset, \emptyset)=\mu_{T} \stackrel{i_{T}}{\rightarrow} \mu$ é o tipo trivial (em $U$ : praticamente em $U$ ) associado a $\mu$. (iii) 
. $\Xi: G R A \rightarrow$ Typ é o funtor tal que $S=\left(S_{0}, S_{1}, d, c\right) \mapsto \Xi(S)=\left(S_{0}, S_{1}, \emptyset, s_{S}, a_{S}, \emptyset\right)$ onde $\forall f \in S_{1}\left(s_{S}(f)=c(f) \wedge I_{f}=1 \wedge a_{S}(f)=\hat{d}(f): I_{f} \rightarrow S_{0}\right): H=\left(H_{0}, H_{1}\right): S \rightarrow S^{\prime}$ $\mapsto \Xi(H)=\left(H_{0}, H_{1}, \emptyset\right): \Xi\left(S^{\prime}\right) \rightarrow \Xi\left(S^{\prime}\right)$

. ( $\Xi$ se fatora por $T y p_{a l g} \hookrightarrow T y p$ e se restringe aos universos.)

. $\Gamma:$ Typ $\rightarrow G R A$ é o funtor tal que $\mu=\left(S, F, R, s_{F}: a_{F}: a_{R}\right) \mapsto \Gamma(\mu)=(S ; \emptyset, \emptyset: \emptyset)$ $: H=\left(H_{\sigma}: H_{\varphi}, H_{\rho}\right): \mu \rightarrow \mu^{\prime} \mapsto \Gamma(H):\left(H_{\sigma}: \emptyset, \emptyset, \emptyset\right): \Gamma(\mu) \rightarrow \Gamma\left(\mu^{\prime}\right)$. ( $\Gamma$ se fatora por GRA disc $\hookrightarrow G R A$ e se restringe aos universos.)

(iv)

Para cada tipo $\mu=\left(S, F, R, s_{F}, a_{F}, a_{R}\right)$ denotamos $E S T(\mu, S E T)$ a categoria das $S E T$ estruturas de tipo $\mu$ :

. $\operatorname{obj}(E S T(\mu, S E T))=\left\{M=\left(M_{S}, M_{F}, M_{R}\right) \in F u n c(S, V) \times F u n c(F, V) \times F u n c(R, V):\right.$ $\forall f \in F\left(M_{F}(f) \in S E T\left(\prod_{i \in I_{f}} M_{S}\left(a_{F}(f)(i)\right), M_{S}\left(s_{F}(f)\right)\right) \wedge \forall r \in R\left(M_{R}(r)=\left[M(r) \stackrel{M\left(j_{r}\right)}{\longmapsto}\right.\right.\right.$ $\left.\left.\left.\prod_{i \in I_{r}} M_{S}\left(a_{R}(r)(i)\right)\right] \in \operatorname{Sub}\left(\prod_{i \in I_{r}} M_{S}\left(a_{R}(r)(i)\right)\right)\right)\right\}$.

- $\forall M, M^{\prime} \in \operatorname{obj}(\operatorname{EST}(\mu, \operatorname{SET})) \operatorname{EST}(\mu, \operatorname{SET})\left(M, M^{\prime}\right)=\{\xi \in F u n c(S, V): \forall s \in$ $S\left(\xi_{s} \in S E T\left(M_{S}(s) ; M_{S}^{\prime}(s)\right)\right) \wedge \forall f \in F\left(\left(\prod_{i \in I_{f}} M_{S}\left(a_{F}(f)(i)\right) \stackrel{M_{F}(f)}{\rightarrow} M_{S}\left(s_{F}(f)\right) \stackrel{\xi_{s_{F}}(f)}{\rightarrow}\right.\right.$ $\left.\left.\prod_{i \in I_{f}} M_{S}^{\prime}\left(a_{F}(f)(i)\right)\right)=\left(\prod_{i \in I_{f}} M_{S}\left(a_{F}(f)(i)\right) \stackrel{\prod_{i \in I_{f}} \xi_{a_{F}(f)(i)}}{\longrightarrow} \prod_{i \in I_{f}} M_{S}^{\prime}\left(a_{F}(f)(i)\right)\right)\right) \wedge \forall r \in R\left(\exists \gamma_{r} \in\right.$ $S \operatorname{ET}\left(M(r), M^{\prime}(r)\right)\left(\left(M(r) \stackrel{\gamma_{r}}{\rightarrow} M^{\prime}(r) \stackrel{M^{\prime}\left(j_{r}^{\prime}\right)}{\longmapsto} \prod_{i \in I_{r}} M_{S}^{\prime}\left(a_{R}(r)(i)\right)\right)=\left(M(r) \stackrel{M\left(j_{r}\right)}{\longrightarrow} \prod_{i \in I_{r}} M_{S}\left(a_{R}(r)(i)\right)\right.\right.$ $\prod_{i \in I_{r}} \xi_{a_{R}(r)(1)}$

$$
\left.\left.\left.\prod_{i \in I_{r}} M_{S}^{\prime}\left(a_{R}(r)(i)\right)\right)\right)\right\}
$$

Observação 1 :

(i) Se $U_{0} \in U_{1} \in U_{2} \in V$ são universos com cardinais $\theta_{0}<\theta_{1}<\theta_{2}<\infty$; obtemos $\operatorname{EST}\left(\right.$, $\left.U_{0}-S E T\right) \in U_{2}-C A T\left(\left(U_{0}-T y p\right)^{\star}, U_{1}-C A T\right)$.

(ii) $\operatorname{EST}\left(\mu, U_{0}-S E T\right) \cong\left(U_{0}-S E T\right)^{\Gamma(\mu)}$ naturalmente em $\mu \in$ obj $\left(\left(U_{0}-T y p_{\text {triv }}\right)^{\star}\right)$; $\left(U_{0}-S E T\right)^{S} \cong E S T\left(\Xi(S), U_{0}-S E T\right)$ naturalmente em $S \in \operatorname{obj}\left(\left(U_{0}-G R A\right)^{\star}\right)$.

(iii) Seja $E S T(\mu, S E T)_{d i s j}$ a subcategoria plena de $E S T(\mu, S E T)$ com objetos a classe das $\mu$-estruturas de "fibras disjuntas" : $\forall s, s^{\prime} \in S\left(M_{S}(s) \cap M_{S}\left(s^{\prime}\right)=\emptyset\right)$ então $\operatorname{EST}(\mu, S E T)_{d i s j}$

$\stackrel{\simeq}{\leftrightarrows} \operatorname{EST}(\mu, S E T)$.

(iv) Denotaremos, para cada $\mu$-estrutura $M,|M|=\coprod_{s \in S} M_{S}(s)$ e $\# M=\#|M|$ (a cardinalidade de $M$ ).

(v) Utilizaremos simplificações notacionais :

- Omitiremos os índices $\sigma: \phi: \rho$ em morfismos de tipos e os índices $S, F, R$ em estruturas.

- Se $M \stackrel{\xi}{\rightarrow} M^{\prime} \in E S T(\mu, S E T)_{d i s j}$ então não faremos distinção notacional com sua reunião (já disjunta!) : $\cup_{s \in S} \xi(s): \bigcup_{s \in S} M(s) \rightarrow \bigcup_{s \in S} M^{\prime}(s)$. Adotaremos convenção semelhante 
para a categoria $\left(S E T^{S}\right)_{\text {disj }}$ onde $S$ é um grafo discreto.

Proposição 1 : "Valorações de variáveis se estendem aos termos" : Para todo tipo pequeno $\mu$ o funtor de esquecimento $\left(\operatorname{EST}(\mu, S E T) \stackrel{\mathcal{E}_{\mu}}{\longrightarrow} S E T^{\Gamma(\mu)}\right)=\left(\operatorname{EST}(\mu, S E T) \stackrel{\text { EST }\left(i_{T}, S E T\right)}{\longrightarrow}\right.$ $\left.\operatorname{EST}\left(\mu_{T}, S E T\right) \stackrel{\cong}{\rightrightarrows} E T^{\Gamma(\mu)}\right)$ admite adjunto à esquerda $S E T^{\Gamma(\mu)} \stackrel{\mathcal{T}_{\mu}}{\rightarrow} \operatorname{EST}(\mu, S E T)$, o funtor " $\mu_{A}$-álgebra dos termos".

Seja $X \in \operatorname{obj}\left(S E T^{\Gamma(\mu)}\right)$.

(i) Basta mostrarmos a propriedade universal quando $X$ "são variáveis" : $X$ tem fibras disjuntas (assim definimos $\left.s_{X}(x)=s \Longleftrightarrow x \in X(s)\right)$ e $\forall s \in S \forall x \in X(s) \forall f \in F($ $\left.s_{F}(f)=s \Rightarrow \forall a \in V(x \neq(f, a))\right)$.

Pois $S: F \in V \Rightarrow \forall s \in S\left(\exists z^{s} \in V\left(z^{s} \notin\left\{f \in F: s_{F}(f)=s\right\} \wedge \forall s, s^{\prime} \in S\left(z^{s} \neq z^{s^{\prime}}\right)\right)\right.$ $\left.\wedge X(s) \cong\left\{\left(z^{s}, x\right): x \in X(s)\right\}\right)$.

(ii) Com a hipótese acima definimos, por indução em $\alpha \in O R D^{+}$, a seqüência crescente de objetos de SET constuituídos por pares ordenados :

$$
\begin{aligned}
& X_{0}=|X|=\bigcup_{s \in S}\left\{(x, s): s_{X}(x)=s\right\} . \\
& . X_{\alpha+1}=X_{\alpha} \cup\left\{\left(\left(f, g r a f\left(T^{\prime}\right)\right), s_{F}(f)\right): f \in F \wedge\left(I_{f} \stackrel{T^{\prime}}{\rightarrow} X_{\alpha} \stackrel{p_{3}}{\rightarrow} S=I_{f} \stackrel{a_{F}(f)}{\rightarrow} S\right)\right\} . \\
& .0 \neq \alpha=\vee\left[0, \alpha\left[\Rightarrow X_{\alpha}=\bigcup_{\beta \in \alpha} X_{\beta} .\right.\right.
\end{aligned}
$$

Então :

. $\forall\left\{(t, s),\left(t, s^{\prime}\right)\right\} \subseteq X_{\infty}\left((t, s)=\left(t, s^{\prime}\right) \Rightarrow s=s^{\prime}\right)$. (Indução em $\alpha \in O R D^{+}$utilizando (i).)

- Dado $(t, s) \in X_{\infty}$ dizemos que $t$ é um $\mu$-termo nas variáveis $X$, com sorte $s\left(s_{T \epsilon r m}(t)=\right.$ $s)$.

. Definimos posto $(t)=\min \left\{\alpha \in O R D^{+}:(t, s) \in X_{\alpha}\right\}$ (é um ordinal sucessor ou 0 ).

. Por indução em posto $(t)$ definimos o conjunto das $X$-variáveis que ocorrem (livres) em $t$ :

.. posto $(t)=0 \Rightarrow \exists(x, s) \in X_{0}\left(\left(t, s_{T \epsilon r m}(t)\right)=(x, s)\right)$ neste caso $X l(t)=\{x\}$.

.. posto $(t)=\alpha+1 \Rightarrow \exists f \in F \exists T^{\prime}: I_{f} \rightarrow X_{\alpha}\left(\left(t, s_{T \epsilon r m}(t)\right)=\left(\left(f, g r a f\left(T^{\prime}\right)\right), s_{F}(f)\right)\right)$ neste caso $X l(t)=\bigcup_{i \in I_{f}} X l\left(p_{1}(T(i))\right)$.

. Seja $\pi=\pi\left(\mu_{A}\right)$ então $\# X l(t)<\pi$ ( indução em posto $\left.(t)\right)$ e $X_{\pi}=X_{\infty}$ ( $\pi$ é cardinal regular $\left.\Rightarrow X_{\pi+1} \subseteq X_{\pi}\right)$.

. Sejam $0 \leq \pi=r \epsilon g(\pi) \leq \tau<\propto$ tais que $\pi(\mu) \leq \pi: \tau(\mu) \leq \tau$. Se \#X $\leq \tau^{<\pi}$ então por indução : utilizando o ítem (1.1.3): obtemos que \# $p_{1}\left(X_{\infty}\right)=\# X_{\infty} \leq \tau^{<\pi}$.

(iii)

. Definimos a $\mathcal{T}_{\mu}(X)=\operatorname{Term}(\mu, X)$ como a $\mu_{A}$-estrutura tal que:

.. $\forall s \in S\left(\mathcal{T}_{\mu}(X)(s)=\left\{t: t\right.\right.$ é termo $\left.\wedge s_{T \epsilon r m}(t)=s\right\}$.

..$\forall f \in F\left(\mathcal{T}_{\mu}(X)(f): \prod_{i \in I_{f}} \mathcal{T}_{\mu}(X)\left(a_{F}(f)(i)\right) \rightarrow \mathcal{T}_{\mu}(X)\left(s_{F}(f)\right) . T \mapsto\left(f: \operatorname{gra} f\left(T^{\prime}\right)\right)\right.$ onde 
$\left.T^{\prime}: I_{f} \rightarrow X_{\pi}, i \mapsto\left(T(i), s_{T \in r m}(T(i))\right)\right)$.

. Consideramos $\left.X \stackrel{n_{X} X}{\longrightarrow} \mathcal{E}_{\mu}(T \operatorname{term}(\mu, X))\right)$.

(iv) $\eta_{X}$ é universal: $\forall M \in o b j(E S T(\mu, S E T)) \forall v \in S E T^{\Gamma(\mu)}\left(X, \mathcal{E}_{\mu}(M)\right) \exists ! v^{\prime} \in \operatorname{EST}(\mu, S E T)$ $(T \in \operatorname{rrm}(\mu, X), M)\left(\mathcal{E}_{\mu}\left(v^{\prime}\right) \circ \eta_{X}=v\right)$ :

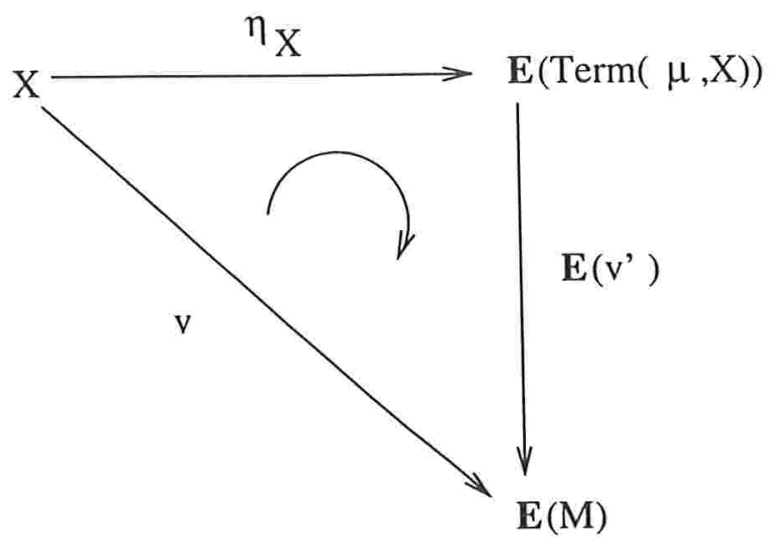

Definimos $v^{\prime}$ por indução no posto dos termos:

. posto $(t)=0 \Rightarrow \exists x \in X(t=x)$. Definimos $v^{\prime}(x)=v(x)$.

. posto $(t)=\alpha+1 \Rightarrow \exists f \in F \exists T: I_{f} \rightarrow T \epsilon r m(\mu, X)\left(a_{F}(f)=s_{T \epsilon r m} \circ T \wedge \forall i \in I_{f}(\right.$ $\operatorname{posto}(T(i)) \leq \alpha) \wedge$ " $t=f(T)$ "). Definimos $v^{\prime}(f(T))=M(f)\left(\left(v^{\prime}(T(i))_{i \in I_{f}}\right)\right.$.

Deste modo $v^{\prime}$ satisfaz os requisitos acima.

\section{Observação 2 :}

(i) Para todo tipo $\mu=\left(S, F, R, s_{F} ; a_{F}, a_{R}\right)$ a propriedade universal acima nos diz que toda $\mu$-estrutura $M$ é naturalmente uma $\mu_{X}$-estrutura onde:

. $\mu_{X}=\left(S, T \epsilon r m(\mu, X), R, s_{T e r m}, a_{T \epsilon r m}, a_{R}\right)$ :

.. $a_{T e r m}(t): \# X l(t) \cong X l(t) \rightarrow S, x \mapsto s_{X}(x)$.

. $M_{X}(t): \prod_{x \in X l(t)} M\left(a_{\text {Term }}(t)(x)\right) \rightarrow M\left(s_{T \in r m}(t)\right):\left.v\right|_{X l(t)} \mapsto v^{\prime}(t)$.

Ou seja:

. Se $t=x \in X$ então $M_{X}(t)=1_{M\left(s_{X}(x)\right)}: M\left(s_{X}(x)\right) \rightarrow M\left(s_{X}(x)\right)$

. Se $t=f(T)$ então ${ }^{1}$ :

\footnotetext{
${ }^{1}$ Nos diagramas desta observação reservaremos a letra $p$ : munida de índices, para indicar "projeções ".
} 


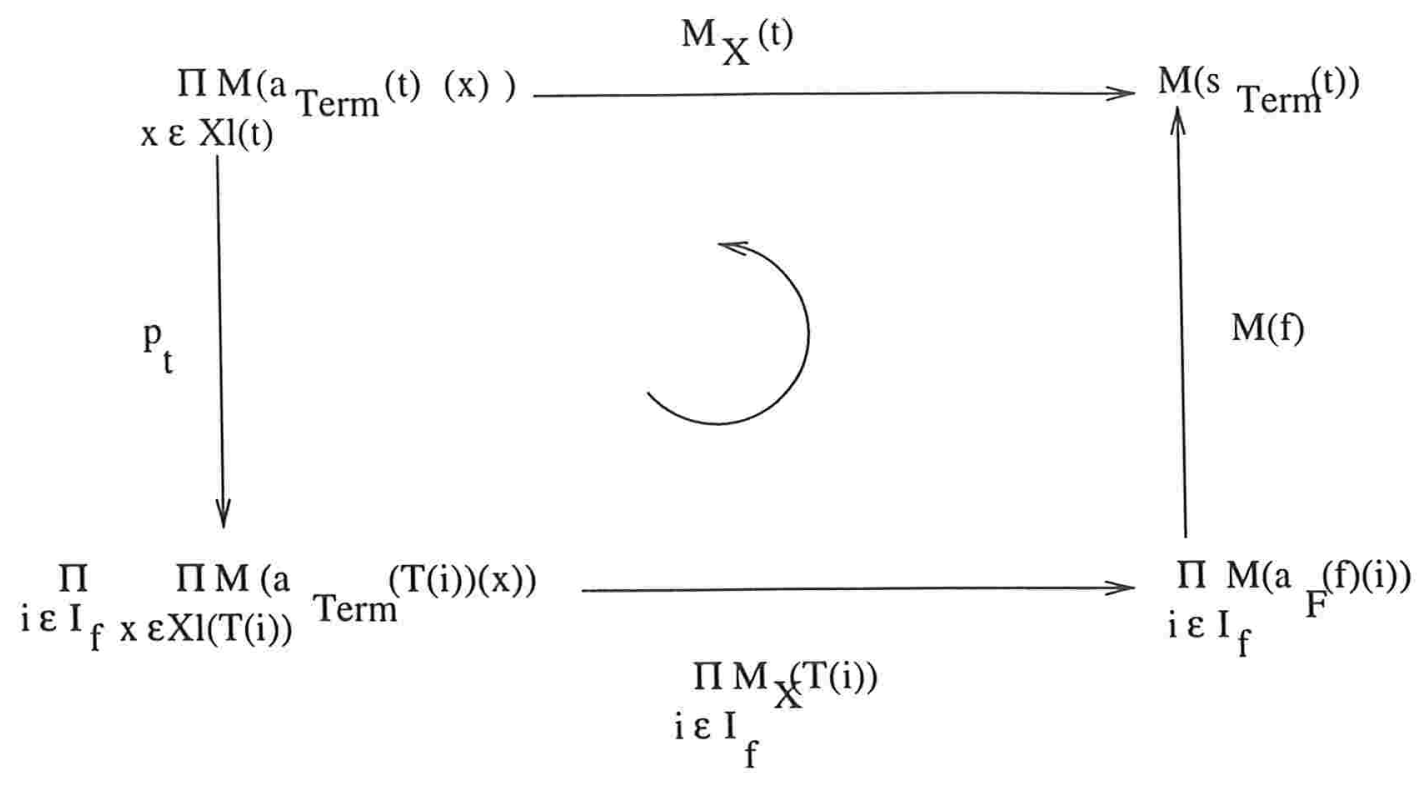

Onde $p_{T}$ é tal que :

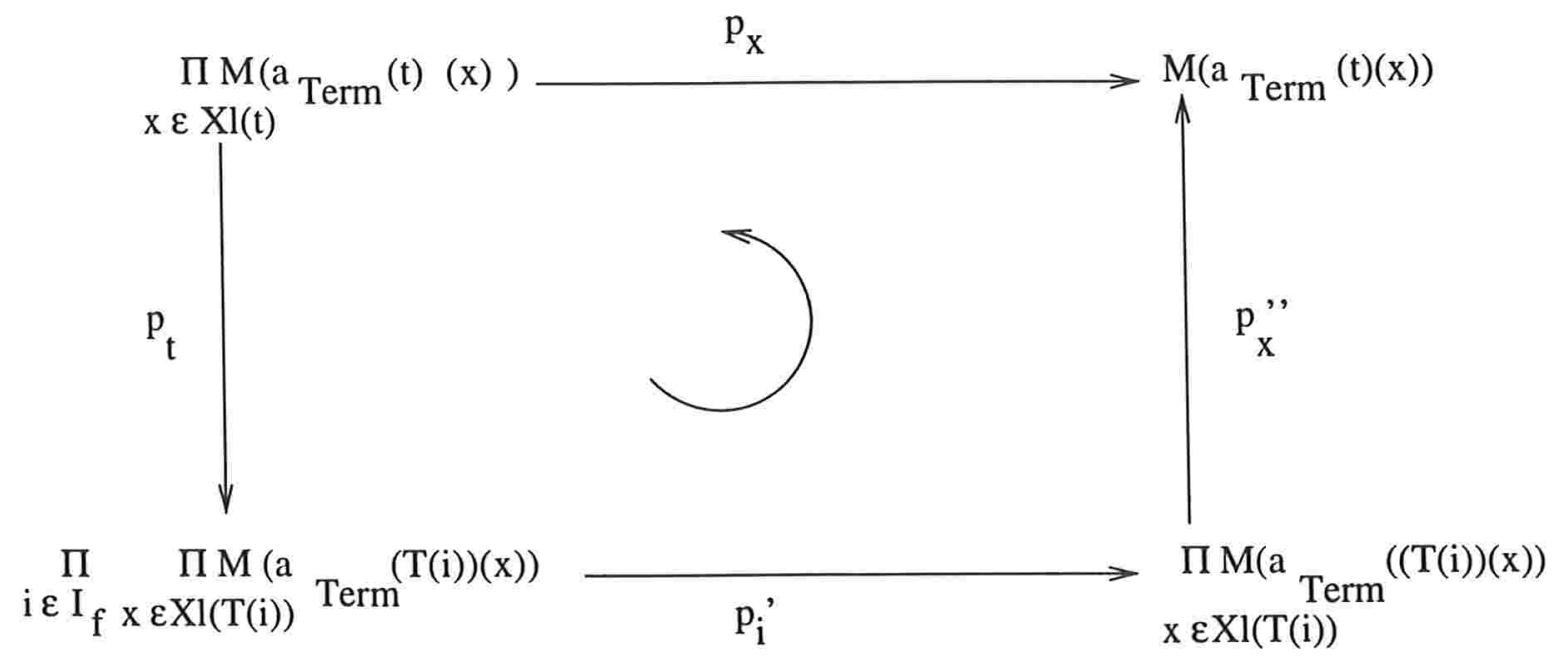

(ii) A proposição 1 e o resultado acima se "restringem aos universos" : $\mu \in V \leadsto \mu \in U_{0}$ :SET $\leadsto U_{0}-S E T$. (Subtituir $V \leadsto U_{0}$ e $\propto \rightsquigarrow \theta_{0}$.)

Definição 2 : Sejam $\mu$ tipo : $\lambda$, $\pi$ cardinais regulares infinitos tais que $\propto>\lambda \geq \pi \geq \pi(\mu)$ e $X$ uma escolha de variáveis tal que $\forall s \in S(\# X(s)=\lambda)$. Consideremos os símbolos: ${ }^{2}$ $=, \wedge, \vee, \neg: \rightarrow, \forall ; \exists$.

A linguagem $L_{\lambda \pi}(\mu)$ consiste dos objetos abaixo:

(i) $E_{\lambda \pi}(\mu)$ : as expressões de $L_{\lambda \pi}(\mu)$.

${ }^{2}$ Conjuntos dois a dois distintos, distintos dos símbolo relacionais de $\mu$ e que pertencem a todo $U \in U N I V$ 
Definimos indutivamente a seqüência crescente de conjuntos $\left\{E^{\xi}\right\}_{\xi \in O R D^{+}}$:

. $E^{0}=\left\{(=, T): T: 2 \rightarrow T \epsilon r m(\mu, X), s_{T \epsilon r m}(T(0))=s_{T \epsilon r m}(T(1))\right\} \cup\{(r, T): r \in R$. $\left.T: I_{r} \rightarrow T \operatorname{Term}(\mu, X), s_{T \epsilon r m} \circ T=a_{R}(r)\right\}$.

. $E^{\xi+1}=E^{\xi} \cup\left\{(\neg: \Psi): \Psi: 1 \rightarrow E^{\xi}\right\} \cup\left\{(\rightarrow, \Psi): \Psi: 2 \rightarrow E^{\xi}\right\} \cup\left\{(\wedge, \Psi): \Psi: \beta \rightarrow E^{\xi}\right.$. $\beta<\lambda\} \cup\left\{(\vee, \Psi): \Psi: \beta \rightarrow E^{\xi}: \beta<\lambda\right\} \cup\left\{(\forall,(Z, \Psi)): \Psi: 1 \rightarrow E^{\xi}: Z: \beta \rightarrow X, \beta<\pi\right\}$ $\cup\left\{(\exists,(Z, \Psi)): \Psi: 1 \rightarrow E^{\xi}, Z: \beta \rightarrow X: \beta<\pi\right\}$.

$0 \neq \xi=\sup \left[0, \xi\left[\Rightarrow E^{\xi}=\bigcup_{\zeta<\xi} E^{\zeta}\right.\right.$.

Definimos $E_{\lambda \pi}(\mu)=E^{\infty}$. Para $\psi \in E_{\lambda \pi}(\mu)$ definimos posto $(\psi)=\min \left\{\xi \in O R D^{+}: \psi \in\right.$ $\left.E^{\xi}\right\}$.

(ii) Análogamente, definimos indutivamente as noções de subexpressão $(S u b(\psi))$; de $X$-variáveis ocorrendo numa expressão $(X(\psi) \subseteq|X|)$, de $X$-variáveis ocorrendo livremente numa expressão $(X l(\psi)) \ldots$

(iii) $F_{\lambda \pi}(\mu)$ : a classe das fórmulas em $L_{\lambda \pi}(\mu)$.

Dizemos que uma expressão $\psi$ é fórmula $\left.\left(\psi \in F_{\lambda \pi}(\mu)\right) \Longleftrightarrow \# X l(\psi)<\pi\right)$. Em particular, dizemos que $\psi$ é sentença quando $X l(\psi)=\emptyset$.

Observação 3 : Por indução no posto das expressões verificamos que:

(i) Expressões de posto zero (atômicas) são fórmulas.

(ii) Subexpressões de fórmulas são fórmulas.

(iii) Toda fórmula é subfórmula de uma sentença .

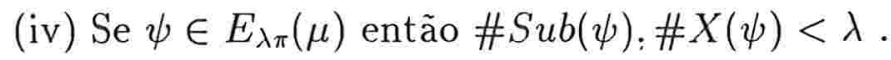

(v) $E_{\lambda}=E_{\infty}$.

(vi) Se $\lambda \geq \tau(\mu)$ então \# $E_{\lambda \pi}(\mu) \leq \lambda^{<\pi}$. ( ítem (1.1.3) )

\section{Observação 4 :}

(i) Utilizaremos as simplificações :

. Notacionais : $r(T), \psi_{0} \rightarrow \psi_{1}, \underset{\alpha \in \beta}{\vee} \psi_{\alpha} ; \exists\left(x_{\alpha}\right)_{\alpha \in \beta} \psi_{0} ; \cdots$

. "Semanticamente orientadas" :

.. Aplicamos os operadores $\wedge, \vee$ em conjuntos de fórmulas .

.. Aplicamos os operadores $\forall, \exists$ em conjuntos de variáveis e a uma fórmula .

- Se $\mu$ é um tipo praticamente em $U=V_{\theta} \in U N I V$ : escolheremos variáveis $X$ para $L_{\theta \theta}(\mu)$ tal que $\forall s \in S(X(s) \subseteq U)$.

(ii) $\psi_{0} \leftrightarrow \psi_{1} \leftrightarrow\left(\psi_{0} \rightarrow \psi_{1}\right) \wedge\left(\psi_{1} \rightarrow \psi_{0}\right)$.

(iii) $T \underset{\emptyset}{\wedge} ; \perp \underset{\emptyset}{\bigvee}$.

(iv) $\exists$ ! $\left(x_{\alpha}\right)_{\alpha \in \beta} \psi_{0}$ m $\exists\left(x_{\alpha}\right)_{\alpha \in \beta} \psi_{0} \wedge \forall\left(x_{\alpha}^{\prime}\right)_{\alpha \in \beta} \forall\left(x_{\alpha}^{\prime \prime}\right)_{\alpha \in \beta}\left(\psi_{0}^{\left({ }^{\prime}\right)} \wedge \psi_{0}^{\left({ }^{\prime \prime}\right)} \rightarrow \underset{\alpha \in \beta}{\wedge} x_{\alpha}^{\prime}=x_{\alpha}^{\prime \prime}\right)$.

(v) $\underset{\alpha \in \beta}{\sqcup} \psi_{\alpha}=\left(\underset{\alpha \in \beta}{\vee} \psi_{\alpha}\right) \wedge\left(\wedge_{\alpha \neq \alpha^{\prime}}^{\wedge}\left(\neg \psi_{\alpha} \wedge \neg \psi_{\alpha^{\prime}}\right)\right)$.

Definição 3 : "Verdade de Tarski" : Sejam $\mu \in \operatorname{obj}\left(U_{0}-T y p\right), M \in \operatorname{obj}\left(\operatorname{EST}\left(\mu, U_{0}-\right.\right.$ $S E T)$ e $X$ um conjunto de variáveis em $U_{0}-S E T$ para $L_{\theta_{0} \theta_{0}}(\mu)$. Definiremos indutivamente; 
para cada fórmula $\psi$, subobjetos $\left[M_{X}(\psi) \stackrel{M_{X}\left(j_{\psi}\right)}{\rightarrow} \prod_{x \in X l(\psi)} M\left(s_{X}(x)\right)\right]$ tais que se $M$ tem fibras disjuntas então para cada valoração $v$,definida num $U_{0}$-subconjunto de $X$ contendo $X l(\psi)$ ; na estrutura $M$ : temos $\left.M \underset{v}{\vDash} \psi \Longleftrightarrow v\right|_{X l(\psi)} \in M_{X}(\psi) \leq \prod_{x \in X l(\psi)} M\left(s_{X}(x)\right)$.

Para cada termo $t$ nas variáveis $X^{\prime} \in \operatorname{obj}\left(\left(U_{0}-S E T\right)^{\Gamma(\mu)}\right) \operatorname{com}\left|X^{\prime}\right| \subseteq|X|$, consideramos como na observação (2.i) a operação derivada $M_{X}(t): \prod_{x \in X l(t)} M\left(a_{T \epsilon r m}(t)(x)\right) \rightarrow M\left(s_{T \epsilon r m}(t)\right)$

(i) $\psi$ m $t_{0}=t_{1}$

$M_{X}(\psi)=\left\{\left(m_{s_{X}(x)}\right)_{x \in X l(\psi)}: M_{X}\left(t_{0}\right)\left(\left(m_{s_{X}(x)}\right)_{x \in X l\left(t_{0}\right)}\right)=M_{X}\left(t_{1}\right)\left(\left(m_{s_{X}(x)}\right)_{x \in X l\left(t_{1}\right)}\right)\right\}$.

Ou seja:

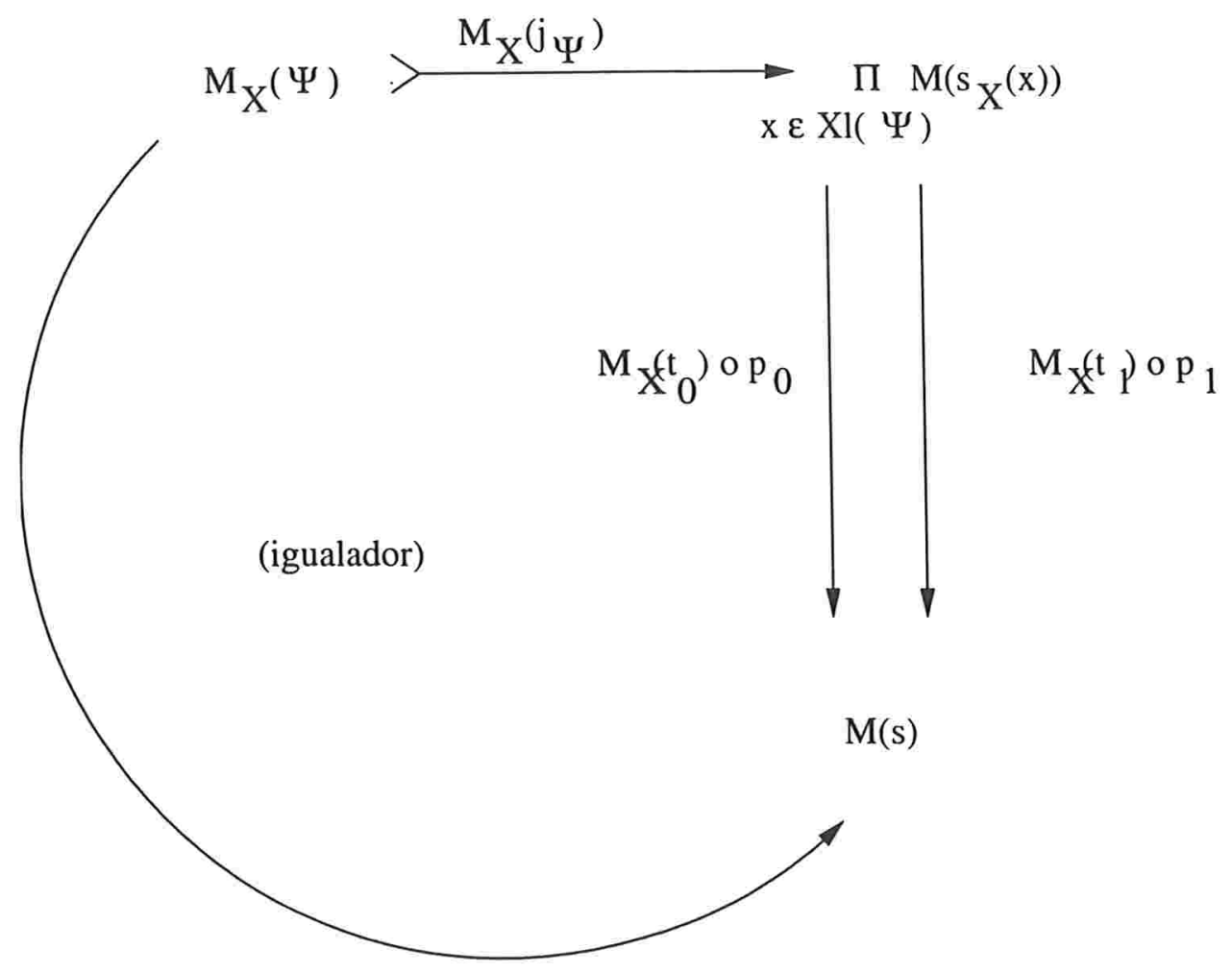

(ii) $\psi \min r(T)$

$M_{X}(\psi)=\left\{\left(m_{s_{X}(x)}\right)_{x \in X l(\psi)}:\left(M_{X}(T(i))\left(\left(m_{s_{X}(x)}\right)_{x \in X l(T(i))}\right)\right)_{i \in I_{r}} \in M(r)\right\}$.

Ou seja: 


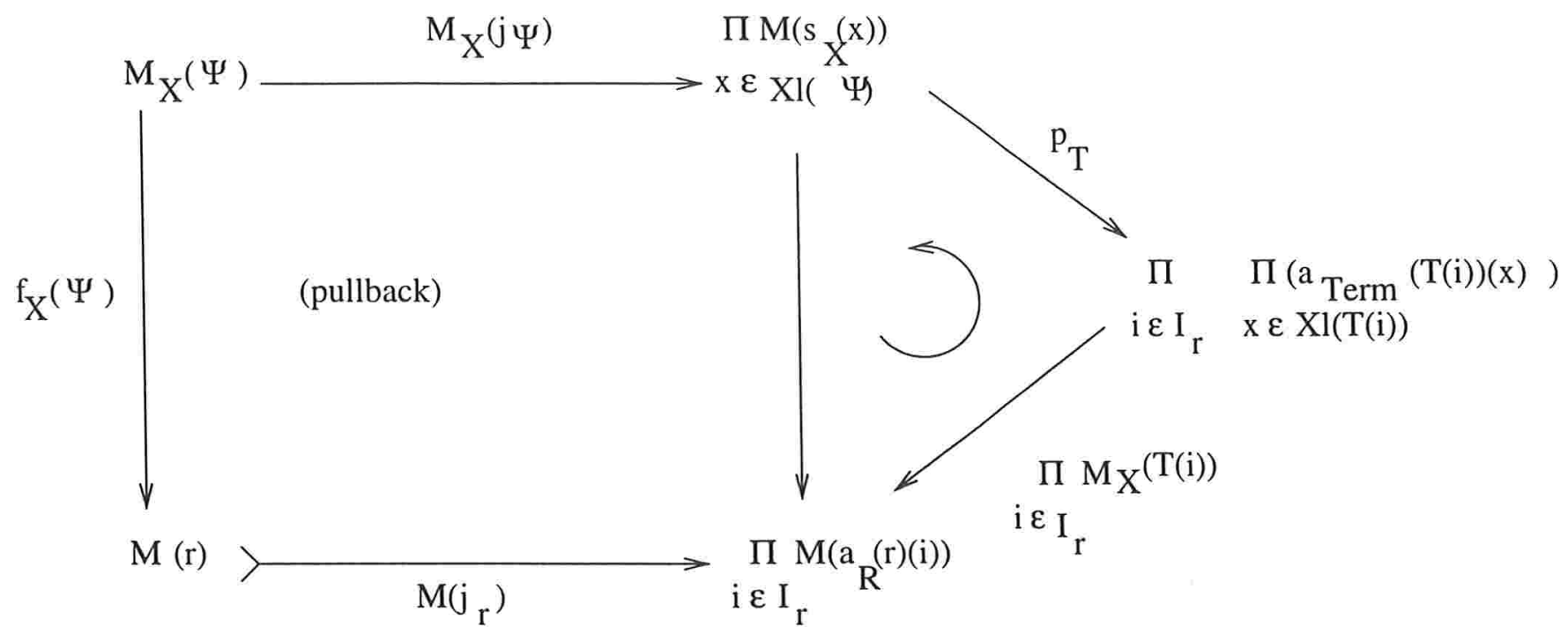

Onde $p_{T}$ é tal que :

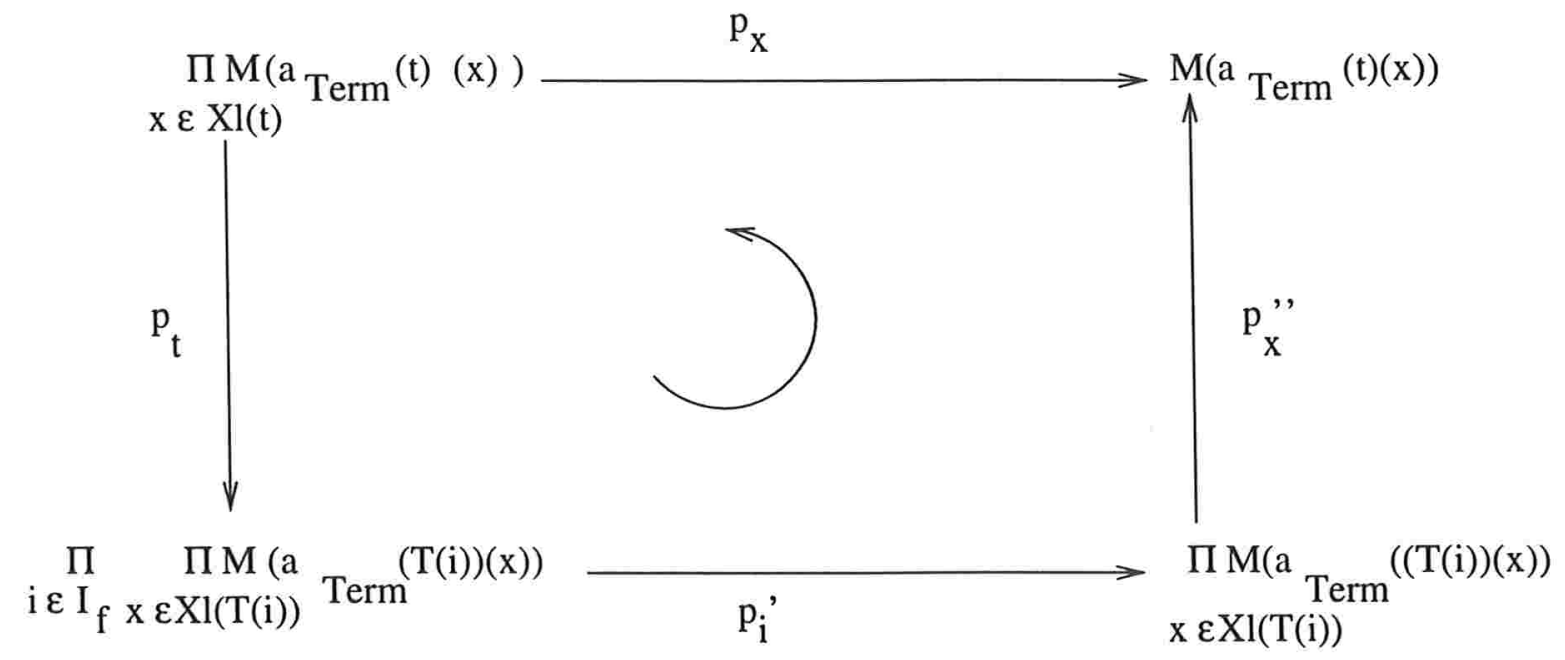

(iii) $\psi$ ans $\bigwedge_{\alpha \in \beta} \psi_{\alpha}$

Para cada $\alpha \in \beta$ consideremos o seguinte "pullback" : $M_{X}^{\psi}\left(\psi_{\alpha}\right)=\left\{\left(m_{s_{X}(x)}\right)_{x \in X l(\psi)}\right.$ $\left.\in \prod_{x \in X l(\psi)} M\left(s_{X}(x)\right):\left(m_{s_{X}(x)}\right)_{x \in X l\left(\psi_{\mathrm{a}}\right)} \in \prod_{x \in X l\left(\psi_{\mathrm{a}}\right)} M\left(s_{X}(x)\right)\right\}$.

Então :

$M_{X}(\psi)=\left\{\left(m_{s_{X}(x)}\right)_{x \in X l(\psi)}: \forall \alpha \in \beta\left(\left(m_{s_{X}(x)}\right)_{x \in X l(\psi)} \in M_{X}^{\psi}\left(\psi_{\alpha}\right)\right)\right\}=\bigcap_{\alpha \in \beta} M_{X}^{\psi}\left(\psi_{\alpha}\right)$.

Ou seja: 


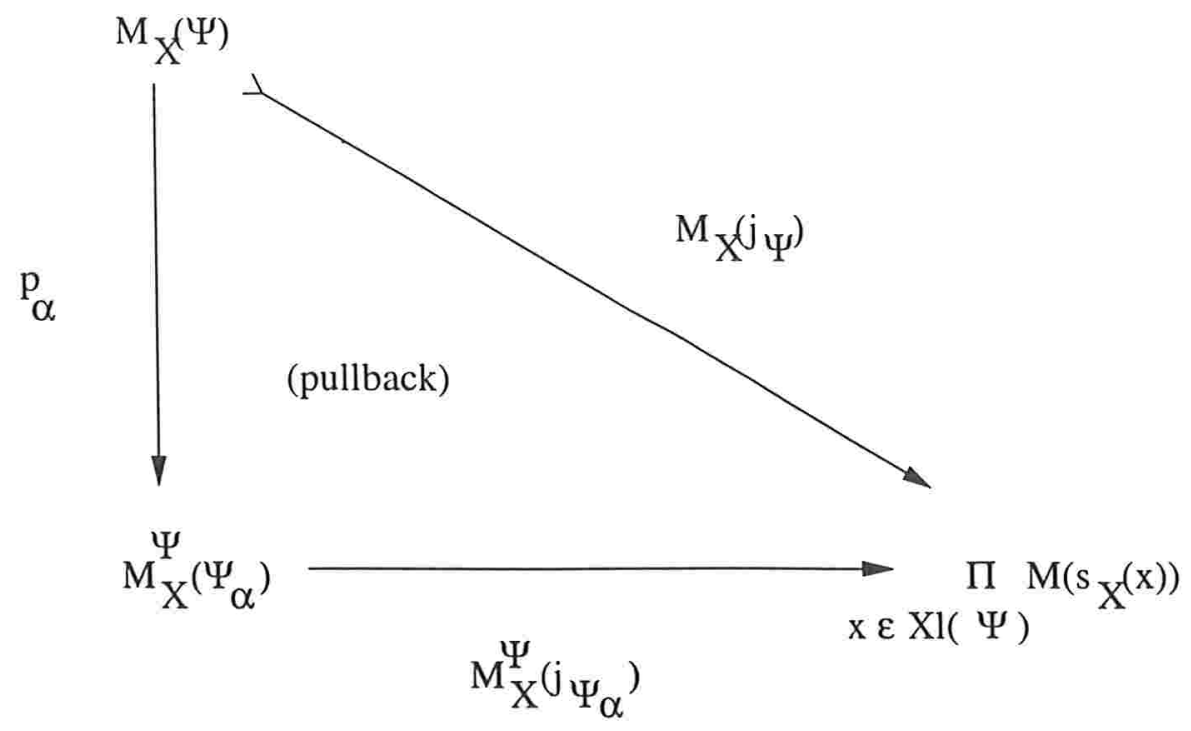

Obs.: Em particular, se $\beta=0$ então $M_{X}(\top)=1=\{\emptyset\}$.

(iv) $\psi m \psi_{0} \rightarrow \psi_{1}$

Para cada $\alpha \in 2$ consideremos o pullback: $M_{X}^{\psi}\left(\psi_{\alpha}\right)=\left\{\left(m_{s_{X}(x)}\right)_{x \in X l(\psi)} \in \prod_{x \in X l(\psi)} M\left(s_{X}(x)\right)\right.$ : $\left.\left(m_{s_{X}(x)}\right)_{x \in X l\left(\psi_{\alpha}\right)} \in \prod_{x \in X l\left(\psi_{\alpha}\right)} M\left(s_{X}(x)\right)\right\}$.

Então :

$M_{X}(\psi)=\left\{\left(m_{s_{X}(x)}\right)_{x \in X l(\psi)}:\left(m_{s_{X}(x)}\right)_{x \in X l(\psi)} \in M_{X}^{\psi}\left(\psi_{0}\right) \Rightarrow\left(m_{s_{X}(x)}\right)_{x \in X l(\psi)} \in M_{X}^{\psi}\left(\psi_{1}\right)\right\}$

Equivalentemente, existe (automaticamente único e monomorfismo!) $f_{X}(\psi): M_{X}^{\psi}\left(\psi_{0}\right) \rightarrow$ $M_{X}^{\psi}\left(\psi_{1}\right)$ tal que:

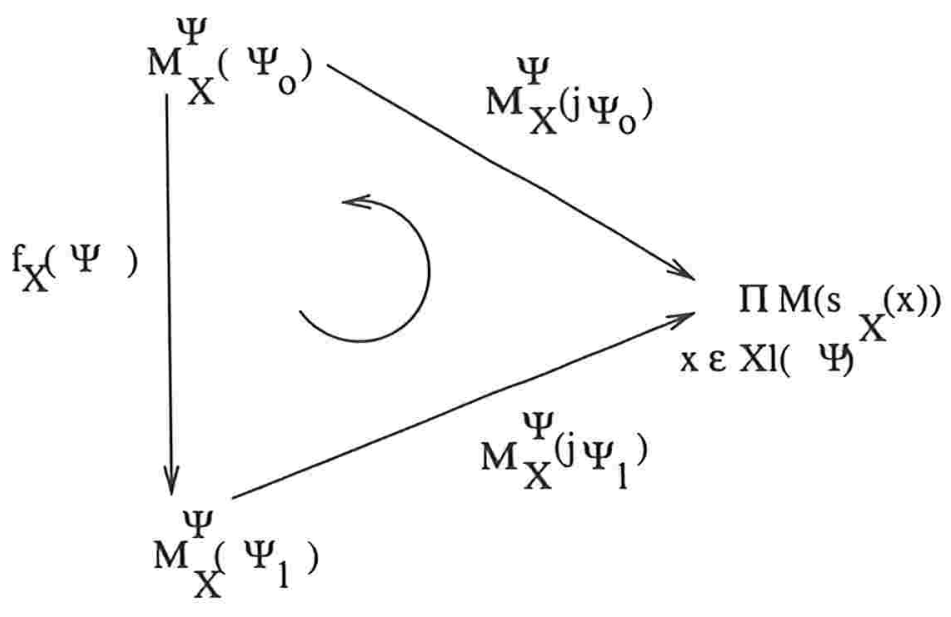

$$
\begin{aligned}
& (\mathrm{v}) \psi m \forall\left(x_{\alpha}\right)_{\alpha \in \beta} \psi_{0} \\
& M_{X}(\psi)=\left\{\left(m_{s_{X}(x)}\right)_{x \in X l(\psi)} \in \prod_{x \in X l(\psi)} M\left(s_{X}(x)\right): \forall\left(m_{s_{X}(x)}^{\prime}\right)_{x \in X l\left(\psi_{0}\right)} \in M_{X}\left(\psi_{0}\right)\left(\left(m_{s_{X}(x)}^{\prime}\right)_{x \in X l(\psi)}\right.\right. \\
= & \left.\left.\left(m_{s_{X}(x)}\right)_{x \in X l(\psi)}\right)\right\} . \text { Seja o pullback : } M_{X}^{\psi_{0}}(\psi)=\left\{\left(m_{s_{X}(x)}\right)_{x \in X l\left(\psi_{0}\right)} \in \prod_{x \in X l\left(\psi_{0}\right)} M\left(s_{X}(x)\right):\right.
\end{aligned}
$$


$\left.\left.\left(m_{s_{X}(x)}\right)_{x \in X l(\psi)} \in M_{X}(\psi)\right)\right\}$. Então $M_{X}(\psi)$ é o maior subobjeto de $\prod_{x \in X_{l} l(\psi)} M\left(s_{X}(x)\right)$ tal que existe (automaticamente único e monomorfismo!) $f_{X}(\psi): M_{X}\left(\psi_{0}\right) \rightarrow M_{X}^{\psi_{0}}(\psi)$ tal que:

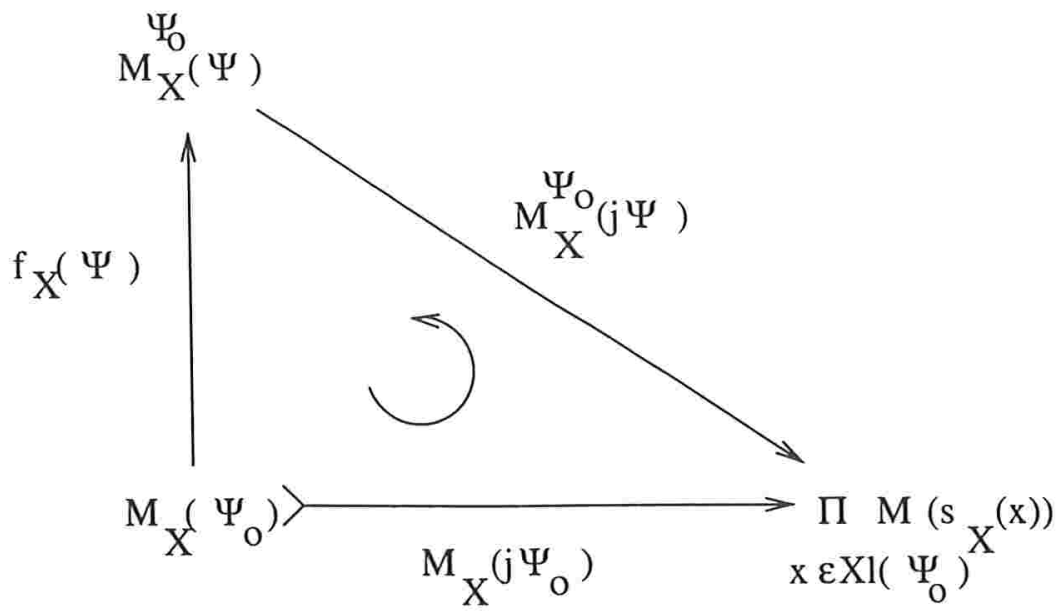

(vi) $\psi m \underset{\alpha \in \beta}{\vee} \psi_{a}$

Para cada $\alpha \in \beta$ consideremos o seguinte "pullback" : $M_{X}^{\psi}\left(\psi_{\alpha}\right)=\left\{\left(m_{s_{X}(x)}\right)_{x \in X l(\psi)}\right.$ $\left.\in \prod_{x \in X l(\psi)} M\left(s_{X}(x)\right):\left(m_{s_{X}(x)}\right)_{x \in X l\left(\psi_{\mathrm{a}}\right)} \in \prod_{x \in X l\left(\psi_{\mathrm{a}}\right)} M\left(s_{X}(x)\right)\right\}$.

Então :

$M_{X}(\psi)=\left\{\left(m_{s_{X}(x)}\right)_{x \in X l(\psi)}: \exists \alpha \in \beta\left(\left(m_{s_{X}(x)}\right)_{x \in X l(\psi)} \in M_{X}^{\psi}\left(\psi_{\alpha}\right)\right)\right\}=\bigcup_{\alpha \in \beta} M_{X}^{\psi}\left(\psi_{\alpha}\right)$.

Equivalentemente, existe epimorfismo regular (função sobrejetora) $f_{X}(\psi): \coprod_{\alpha \in \beta} M_{X}^{\psi}\left(\psi_{\alpha}\right)$ $\rightarrow M_{X}^{\psi}(\psi)$ tal que:

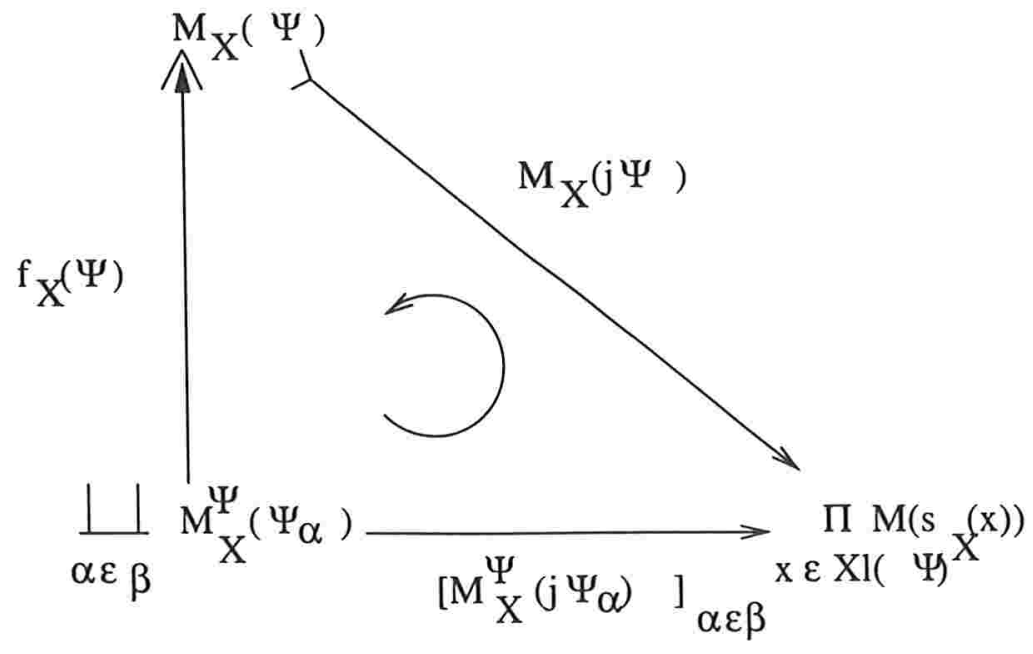

Obs.: Em particular, se $\beta=0$ então $M_{X}(\perp)=\emptyset$.

(vii) $\psi \mathrm{m} \rightarrow \psi_{0}$

Definimos $M_{X}(\psi)=M_{X}\left(\psi_{0} \rightarrow \perp\right)$

(viii) $\psi m \exists\left(x_{\alpha}\right)_{\alpha \in \beta} \psi_{0}$ 
$\begin{aligned} & M_{X}(\psi)=\left\{\left(m_{s_{X}(x)}\right)_{x \in X l(\psi)} \in \prod_{x \in X l(\psi)} M\left(s_{X}(x)\right): \exists\left(m_{s_{X}(x)}^{\prime}\right)_{x \in X l\left(\psi_{0}\right)} \in M_{X}\left(\psi_{0}\right)\left(\left(m_{s_{X}(x)}^{\prime}\right)_{x \in X l(\psi)}\right.\right. \\ = & \left.\left.\left(m_{s_{X}(x)}\right)_{x \in X l(\psi)}\right)\right\} .\end{aligned}$

Equivalentemente, existe epimorfismo regular (função sobrejetora) $f_{X}(\psi): M_{X}\left(\psi_{0}\right) \rightarrow$ $M_{X}(\psi)$ tal que:

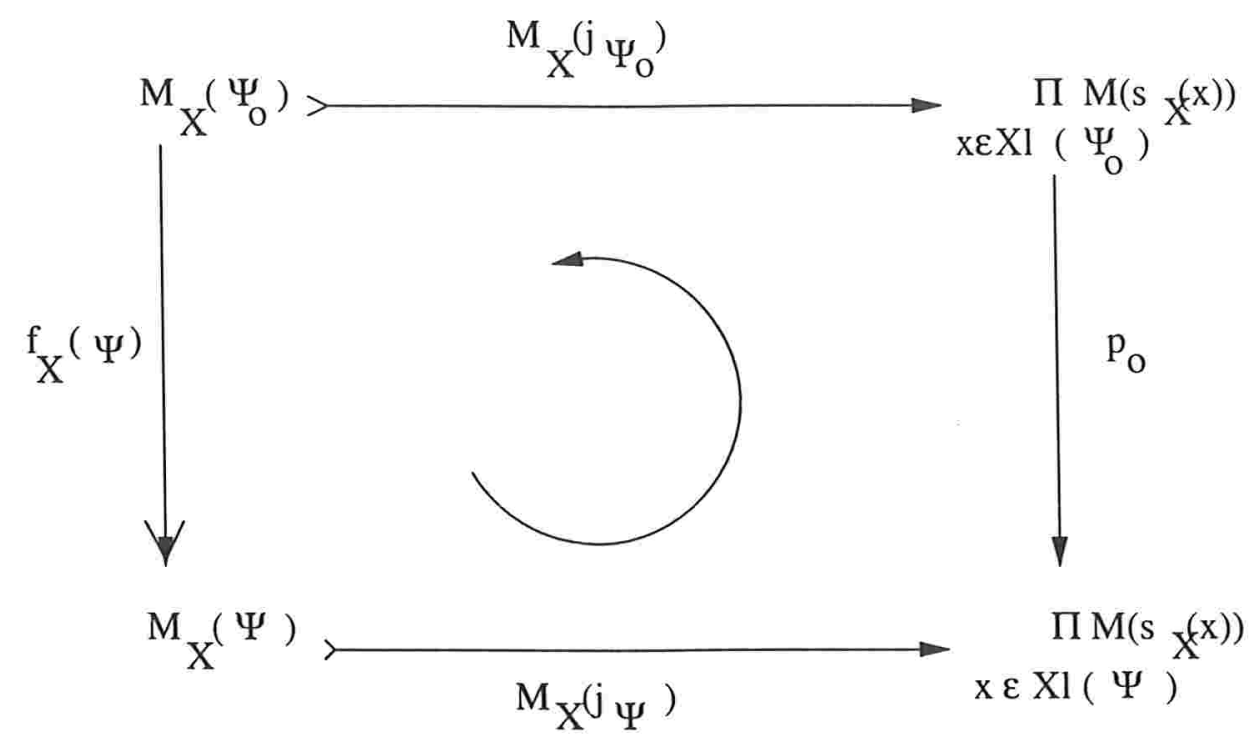

\section{Observação 5 :}

(i) Em particular, quando $\psi$ é sentença $(X l(\psi)=\emptyset)$; temos $M_{X}(\psi) \subseteq 1=\{\emptyset\}$ e $(M$ é modelo de $\psi \Longleftrightarrow$ ) $M \underset{v}{*} \psi \Longleftrightarrow \emptyset=v \mid \emptyset \in M_{X}(\psi) \Longleftrightarrow M_{X}(\psi)=1$.

(ii) Temos $M_{X}(\psi)=M_{X}(\top \rightarrow \psi)$

(iii) Se $\psi$ é sentença equacional, i.e. $\psi$ é sentença da forma $\forall\left(x_{\alpha}\right)_{\alpha \in \beta}\left(t_{0}=t_{1}\right)$ então Mé modelo de $\psi \Longleftrightarrow$

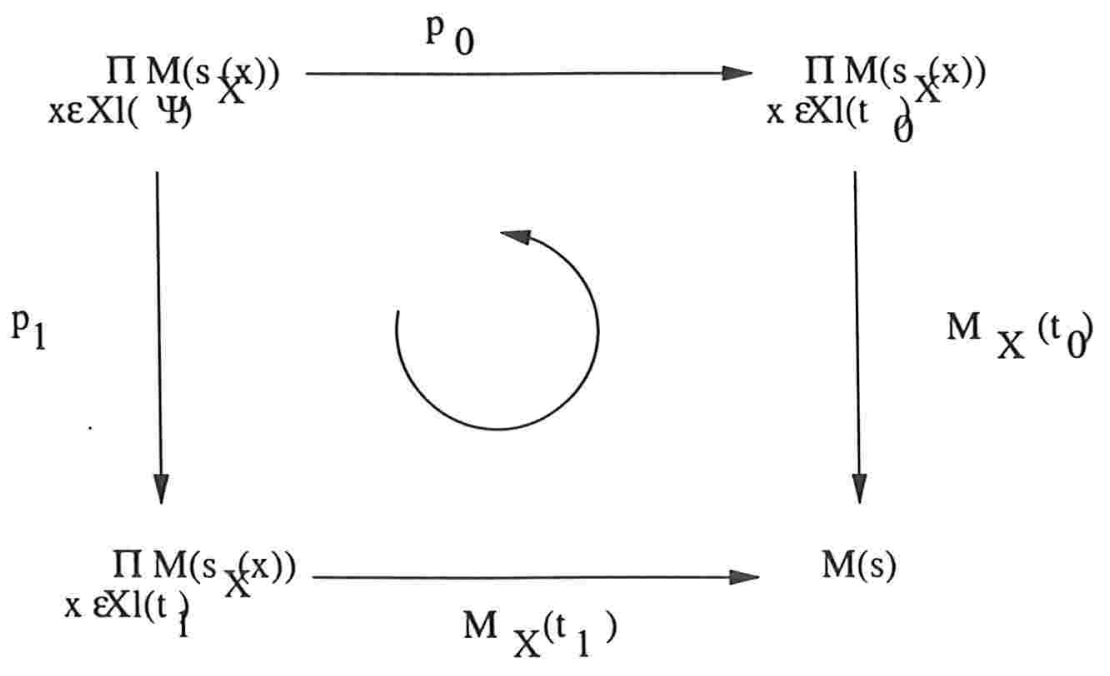

(iv) Se $\psi$ é da forma $\exists$ ! $\left(x_{\alpha}\right)_{\alpha \in \beta} \psi_{0}$ temos: 
$M_{X}(\psi)=\left\{\left(m_{s_{X}(x)}\right)_{x \in X l(\psi)}: \exists !\left(m_{s_{X}(x)}^{\prime}\right)_{x \in X l\left(\psi_{0}\right)} \in M_{X}\left(\psi_{0}\right)\left(\left(m_{s_{X}(x)}^{\prime}\right)_{x \in X l(\psi)}=\left(m_{s_{X}(x)}\right)_{x \in X l(\psi)}\right.\right.$ )\} .

Ou seja, existe isomorfismo $f_{X}(\psi): M_{X}\left(\psi_{0}\right) \stackrel{\cong}{\rightrightarrows} M_{X}(\psi)$ tal que:

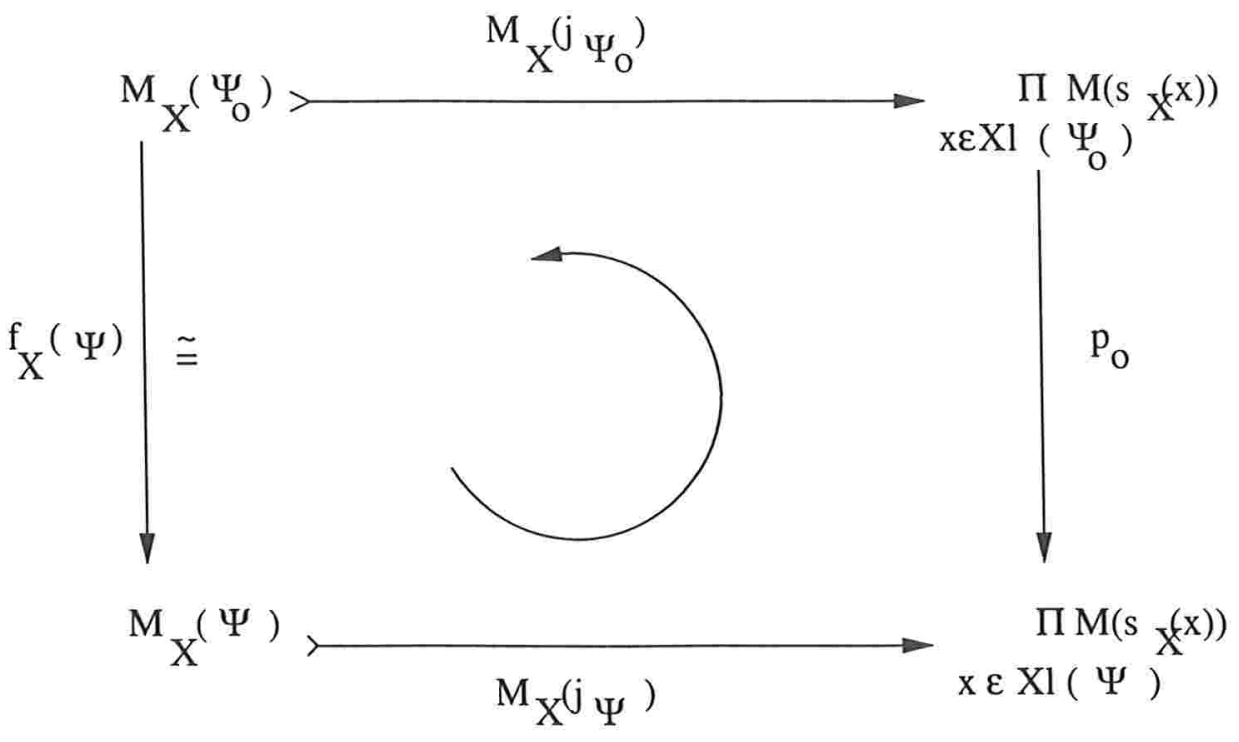

(v) Se $\psi$ é da forma $\underset{\alpha \in \beta}{\sqcup} \psi_{\alpha}$ temos:

$M_{X}(\psi)=\left\{\left(m_{s_{X}(x)}\right)_{x \in X l(\psi)}: \exists ! \alpha \in \beta\left(\left(m_{s_{X}(x)}\right)_{x \in X l(\psi)} \in M_{X}^{\psi}\left(\psi_{\alpha}\right)\right)\right\}=\bigcup_{\alpha \in \beta} M_{X}^{\psi}\left(\psi_{\alpha}\right)$.

Ou seja, existe isomorfismo $f_{X}(\psi): \coprod_{\alpha \in \beta} M_{X}^{\psi}\left(\psi_{\alpha}\right) \stackrel{\cong}{\rightarrow} M_{X}^{\psi}(\psi)$ tal que:

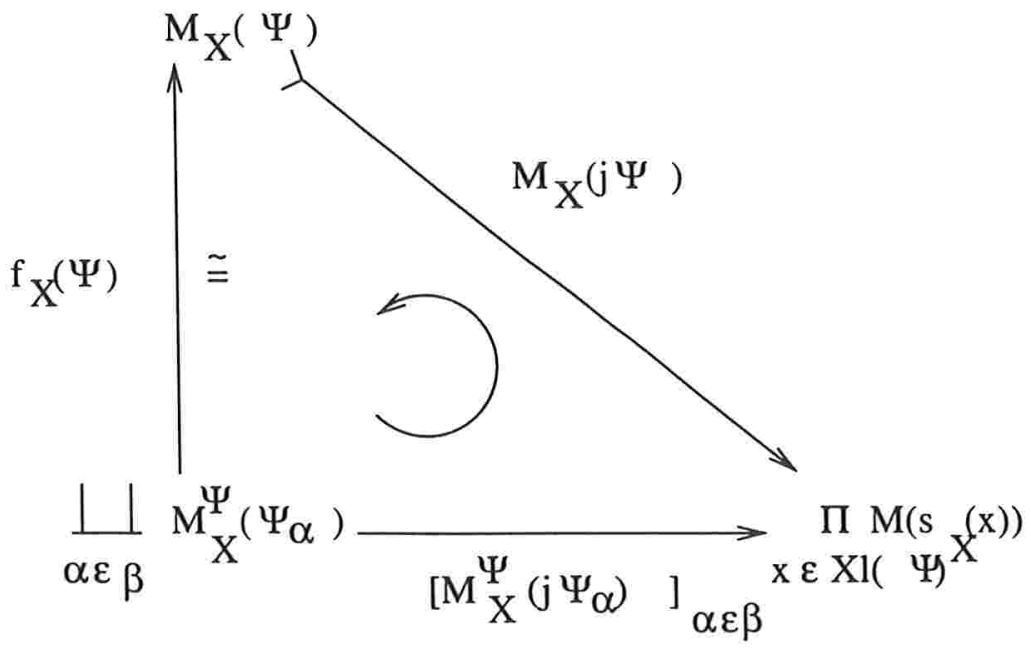

Obs.: Em particular, se $\beta=0$ então $M_{X}(\perp)=\emptyset$.

Definição 4 : Sejam $\mu \stackrel{H}{\rightarrow} \mu^{\prime} \in U_{0}-T y p: X$ e $X^{\prime}$ variáveis em $U_{0}-S E T$ para $L_{\theta_{0} \theta_{0}}(\mu)$ e $L_{\theta_{0} \theta_{0}}\left(\mu^{\prime}\right)$ e $\Gamma:|X| \rightarrow\left|X^{\prime}\right|:$

(i) $\Gamma$ é $H$-morfismo lógico $\Longleftrightarrow s_{X^{\prime}}^{\prime} \circ \Gamma=H \circ s_{X}$.

(ii) Se $\Gamma$ é $H$-morfismo lógico e $Z \subseteq X$ então $\Gamma$ é $Z$-mônico $\Longleftrightarrow \Gamma{ }_{Z}:|Z| \hookrightarrow\left|X^{\prime}\right|$. 
(iii) Se $\Gamma$ é $H$-morfismo lógico denotamos $\hat{\Gamma}$ sua "extensão às expressões" : $\hat{\Gamma}: E_{\theta_{0} \theta_{0}}(\mu) \rightarrow$ $E_{\theta_{0} \theta_{0}}\left(\mu^{\prime}\right)$.

Observação 6 : $E_{\theta_{0} \theta_{0}}(\mu)$ admite uma relação de equivalência natural : $\psi_{0} \sim \psi_{1} \Longleftrightarrow$ existe $\Gamma 1_{\mu}$-isomorfismo lógico $(\Gamma:|X| \cong|\cong| X \mid)$ tal que $\psi_{1}=\hat{\Gamma}\left(\psi_{0}\right)$.

Proposição 2: Sejam $\mu \stackrel{H}{\rightarrow} \mu^{\prime} \in U_{0}-T y p$ : X e $X^{\prime}$ variáveis em $U_{0}-S E T$ para $L_{\theta_{0} \theta_{0}}(\mu)$ e $L_{\theta_{0} \theta_{0}}\left(\mu^{\prime}\right), \psi \in F_{\theta_{0} \theta_{0}}(\mu)$ e $\Gamma H$-morfismo lógico $X(\psi)$-mônico $\left(\# X(\psi)<\theta_{0}\right.$ !). Então para todo $M^{\prime} \in \operatorname{obj}\left(E S T\left(\mu^{\prime}, U_{0}-S E T\right)\right)$; toda $\mu$-valoração $v \operatorname{com} X l(\psi) \subseteq \operatorname{dom}(v)$ temos $\operatorname{EST}\left(H, U_{0}-S E T\right)\left(M^{\prime}\right) \underset{v}{\vDash} \psi \Longleftrightarrow M^{\prime} \underset{\Gamma^{*}(v)}{\vDash} \hat{\Gamma}(\psi)$.

Definição 5 : Sejam $\mu \in \operatorname{obj}\left(U_{0}-T y p\right)$; $X$ variáveis em $U_{0}-S E T$ para $L_{\theta_{0} \theta_{0}}(\mu)$ e $\mathcal{F} \subseteq F_{\theta_{0} \theta_{0}}(\mu):$

(i) $\operatorname{Sejam} M, N \in \operatorname{obj}\left(E S T\left(\mu, U_{0}-S E T\right)\right)$ e $H \in\left(U_{0}-S E T\right)^{\Gamma(\mu)}\left(\mathcal{E}_{\mu}(M), \mathcal{E}_{\mu}(N)\right)$. H é $\mathcal{F}$-morfismo elementar $\Longleftrightarrow$ para todo $\psi \in \mathcal{F}$ e toda $v$ valoração de um $U_{0}$-subconjunto de $X$ contendo $X l(\psi)$ em $M$ temos $M \underset{v}{\vDash} \psi \Rightarrow N \underset{H \circ v}{\vDash} \psi$.

(ii) Denotamos $E S T\left(\mathcal{F}: \mu, U_{0}-S E T\right)$ a categoria das $\mu$-estrutura em $U_{0}-S E T$ e $\mathcal{F}$ morfismos elementares.

(iii) Se $\psi$ é sentença em $L_{\theta_{0} \theta_{0}}(\mu)$ denotamos $\operatorname{Mod}\left(\mathcal{F}, \psi, U_{0}-S E T\right)$ a subcategoria plena de $\operatorname{EST}\left(\mathcal{F}, \mu ; U_{0}-S E T\right)$ com objetos os modelos de $\psi$ em $U_{0}-S E T$.

Observação 7 :

(i) Sejam $\mathcal{F}_{0}, \mathcal{F}_{1} \subseteq F_{\theta_{0} \theta_{0}}(\mu)$ então se imagem $\left(\mathcal{F}_{0} \hookrightarrow F_{\theta_{0} \theta_{0}}(\mu) \rightarrow\left(F_{\theta_{0} \theta_{0}}(\mu)\right) / \sim\right) \subseteq($ $\operatorname{imagem}\left(\mathcal{F}_{1} \hookrightarrow F_{\theta_{0} \theta_{0}}(\mu) \rightarrow\left(F_{\theta_{0} \theta_{0}}(\mu)\right) / \sim\right)$ então $\operatorname{EST}\left(\mathcal{F}_{1}, \mu, U_{0}-S E T\right) \hookrightarrow \operatorname{EST}\left(\mathcal{F}_{0}, \mu, U_{0}-\right.$ $S E T)$.

(ii) $\operatorname{EST}\left(\mathcal{F}^{(\mathrm{\vee})}, \mu, U_{0}-S E T\right)=\operatorname{EST}\left(\mathcal{F}, \mu, U_{0}-S E T\right)=\operatorname{EST}\left(\mathcal{F}^{(\wedge)}, \mu, U_{0}-S E T\right)$.

(iii) Sejam $M, N \in \operatorname{obj}\left(E S T\left(\mu, U_{0}-S E T\right)\right)$ e $H \in\left(U_{0}-S E T\right)^{\Gamma(\mu)}\left(\mathcal{E}_{\mu}(M), \mathcal{E}_{\mu}(N)\right)$ então $H \in E S T\left(\mathcal{F}^{(\neg)}, \mu, U_{0}-S E T\right)(M, N) \Longleftrightarrow$ para todo $\psi \in \mathcal{F}$ e toda $v$ valoração de um $U_{0}$-subconjunto de $X$ contendo $X l(\psi) \operatorname{em} M \operatorname{temos} N \underset{H \circ v}{F} \psi \Rightarrow M \underset{v}{F} \psi$.

(iv) $\operatorname{EST}\left(\operatorname{atom}(\mu), \mu, U_{0}-S E T\right)=\operatorname{EST}\left(\mu, U_{0}-S E T\right)$.

(v) Se $\mathcal{F}=\operatorname{atom}(\mu) \cup(\operatorname{atom}(\mu))^{(\neg)}$ então $\operatorname{EST}\left(\mathcal{F}: \mu, U_{0}-S E T\right)$ é a categoria das $\mu$ estruturas em $U_{0}-S E T$ e mergulhos.

Definição 6 : Sejam $\mu \in \operatorname{obj}\left(U_{0}-T y p\right)$; $X$ variáveis em $U_{0}-S E T$ para $L_{\theta_{0} \theta_{0}}(\mu)$ e $\mathcal{F} \subseteq F_{\theta_{0} \theta_{0}}(\mu)$. $\mathcal{F}$ é essencialmente $\theta_{0}$-pequeno $\Longleftrightarrow$ existe $\overline{\mathcal{F}} \in[\mathcal{F}]^{<\theta_{0}}$ tal que $\overline{\mathcal{F}} \sim$ representa $\mathcal{F}$.

Definição 7 : Sejam $\mu \in \operatorname{obj}\left(U_{0}-T y p\right) ; X$ variáveis em $U_{0}-S E T$ para $L_{\theta_{0} \theta_{0}}(\mu)$ e $\mathcal{F} \subseteq F_{\theta_{0} \theta_{0}}(\mu) . \mathcal{F}$ é um fragmento de $L_{\theta_{0} \theta_{0}}(\mu) \Longleftrightarrow$

. $\mathcal{F} \sim$-representa atom $(\mu)$.

- $\mathcal{F} \sim$-representa as fórmulas obtidas a partir de $\mathcal{F}$ substituindo-se termos por variáveis.

. Para todo $\psi \in \mathcal{F}$ temos $S u b(\psi) \subseteq \mathcal{F}$. 
. Se $\forall\left(x_{\alpha}\right)_{\alpha \in \beta} \psi_{0} \in \mathcal{F}$ então $\neg \exists\left(x_{\alpha}\right)_{\alpha \in \beta} \neg \psi_{0} \in \mathcal{F}$.

. Se $\psi_{0} \rightarrow \psi_{1} \in \mathcal{F}$ então $\neg \psi_{0} \vee \psi_{1} \in \mathcal{F}$.

Observação 8 : Se $\mathcal{F} \subseteq F_{\theta_{0} \theta_{0}}(\mu)$ é essencialmente $\theta_{0}$-pequeno então existe $\overline{\mathcal{F}}$ fragmento essencialmente $\theta_{0}$-pequeno que "contém essencialmente" $\mathcal{F}$.

Exemplos : Nas condições acima $\operatorname{atom}(\mu)$ e $F_{\lambda \pi}(\mu)$ com $\lambda<\theta_{0}$ são fragmentos de $L_{\theta_{0} \theta_{0}}(\mu)$ de cardinalidades $\theta_{0}$ mas essencialmente $\theta_{0}$-pequenos.

Definição 8 : Seja $A \in \operatorname{obj}\left(U_{1}-C A T\right)$. A é categoria axiomatizável $\Longleftrightarrow$ existe $\mu$ tipo praticamente em $U_{0}$, existe $\mathcal{F} \subseteq F_{\theta_{0} \theta_{0}}(\mu)$ fragmento essencialmente $\theta_{0}$-pequeno, existe $\psi$ sentença em $L_{\theta_{0} \theta_{0}}(\mu)$ da forma $(\wedge)(\forall)([(\exists),(\vee),(\wedge), \mathcal{F}] \rightarrow[(\exists):(\vee),(\wedge), \mathcal{F}])$ tal que $A \simeq \operatorname{Mod}\left(\mathcal{F}, \psi, U_{0}-S E T\right)$.

Observação 9 : Claramente, na definição acima, podemos tomar $\mu \in \operatorname{obj}\left(U_{0}-T y p\right)$. Podemos supor $\mathcal{F}=\operatorname{atom}(\mu)$ devido a:

Proposição 3 : Sejam $\mu=\left(S, F, R, s_{F}, a_{F}, a_{R}\right) \in o b j\left(U_{0}-T y p\right), X$ variáveis em $U_{0}-S E T$ para $L_{\theta_{0} \theta_{0}}(\mu)$ e $\mathcal{F} \subseteq F_{\theta_{0} \theta_{0}}(\mu)$ fragmento essencialmente $\theta_{0}$-pequeno. Então existe $\mu^{\prime} \in \operatorname{obj}\left(U_{0}-T y p\right)$ : existe $\psi_{\mathcal{F}}^{\prime}$ sentença em $L_{\theta_{0} \theta_{0}}\left(\mu^{\prime}\right)$ da forma $(\wedge)(\forall)\left(\left[(\exists)\right.\right.$ : $(\vee),(\wedge)$; atom $\left.\left(\mu^{\prime}\right)\right]$ $\left.\rightarrow\left[(\exists),(\vee):(\wedge) ; \operatorname{atom}\left(\mu^{\prime}\right)\right]\right)$ tais que :

. $\mu \hookrightarrow \mu^{\prime}=\left(S, F ; R^{\prime}, s_{F} ; a_{F} ; a_{R^{\prime}}^{\prime}\right)$.

. Para toda $\psi$ sentença em $L_{\theta_{0} \theta_{0}}(\mu)$ da forma $(\wedge)(\forall)([(\exists),(\vee),(\wedge), \mathcal{F}] \rightarrow[(\exists),(\vee),(\wedge), \mathcal{F}])$ existe $\psi^{\prime}$ sentença em $L_{\theta_{0} \theta_{0}}\left(\mu^{\prime}\right)$ da forma $(\wedge)(\forall)\left(\left[(\exists),(\vee),(\wedge)\right.\right.$, atom $\left.\left(\mu^{\prime}\right)\right] \rightarrow\left[(\exists),(\vee) ;(\wedge)\right.$, atom $\left.\left.\left(\mu^{\prime}\right)\right]\right)$ tal que, na $U_{2}$-categoria $\left(\left(U_{1}-C A T\right) \rightarrow \operatorname{EST}\left(\mu_{A}, U_{0}-S E T\right)\right)=\left(\left(U_{1}-C A T\right) \rightarrow \operatorname{EST}\left(\mu_{A}^{\prime}, U_{0}-\right.\right.$ $S E T))$, temos isomorfismo: $\left(\operatorname{Mod}\left(\mathcal{F}, \psi, U_{0}-S E T\right) \hookrightarrow E S T\left(\mu_{A}, U_{0}-S E T\right)\right) \cong\left(\operatorname{Mod}\left(\operatorname{atom}\left(\mu^{\prime}\right)\right.\right.$,

$\left.\left.\psi^{\prime} \wedge \psi_{\mathcal{F}}^{\prime}, U_{0}-S E T\right) \hookrightarrow E S T\left(\mu_{A}^{\prime}, U_{0}-S E T\right)\right)$.

Enunciamos abaixo uma versão do teorema de Löwenheim-Skolem tal que (sua relativização a $\left.U_{0}-S E T\right)$ nos será útil no capítulo seguinte:

Proposição $4: \mathcal{L S} \downarrow$ : Sejam $\mu \in \operatorname{obj}\left(U_{0}-T y p\right): L_{\tau^{+} \pi}(\mu)$ uma lógica com \# $\tau(\mu) \leq \tau<$ $\theta_{0}$ e $\kappa=\left(\tau^{<\pi}\right)^{+}$. Então para toda sentença $\psi$ de $L_{\tau^{+} \pi}(\mu)$, todo $M \in \operatorname{obj}\left(\operatorname{Mod}\left(\operatorname{atom}(\mu), \psi, U_{0}-\right.\right.$ $S E T))$ e todo $Z \in[|M|]^{<\kappa}$ existe $N \in(S u b(M))^{<\kappa}$ tal que $Z \subseteq|N|$. 


\section{Capítulo 5}

\section{Categorias axiomatizáveis, esboçáveis e acessíveis}

Reservamos este capítulo para demonstrar que os conceitos introduzidos anteriormente se correlacionam, ampliando, por assim dizer, o espectro de cada um desses. A referência [166] reune esses resultados.

\subsection{Categorias esboçáveis são axiomatizáveis}

Proposição 1 : Tendo em vista o funtor $\Xi: U_{0}-G R A \rightarrow U_{0}-T y p$ e o isomorfismo natural $\tilde{\Xi}: U_{0}-G R A\left({ }_{-} \mathcal{E}\left(U_{0}-S E T\right)\right) \stackrel{\cong}{\rightrightarrows} S T\left(\Xi\left({ }_{-}\right), U_{0}-S E T\right)$ (definição (1.iii) e observação (1.ii) do capítulo 4); temos que:

(i) $\forall m, n \in \omega \exists \gamma_{G_{m}^{n}}^{o}$ sentença em $L_{\theta_{0} \theta_{0}}\left(\Xi\left(G_{m}^{n}\right)\right)$ da forma $(\forall(<\omega))\left(\operatorname{atom}\left(\Xi\left(G_{m}^{n}\right)\right)\right.$ tal que $\forall d^{\prime} \in U_{0}-G R A\left(G_{m}^{n} ; \mathcal{E}\left(U_{0}-S E T\right)\right.$ ) ( $d^{\prime}$ é diagrama comutativo $\left.\Longleftrightarrow \tilde{\Xi}\left(d^{\prime}\right) \vDash \gamma_{G_{m}^{n}}^{\circ}\right)$.

(ii) $\forall G \in \operatorname{obj}\left(U_{0}-G R A\right) \exists \gamma_{G}^{\downarrow}$ sentença em $L_{\theta_{0} \theta_{0}}\left(\Xi\left(G^{-}\right)\right)$da forma $\left(\wedge_{<\omega}\right)\left(\forall_{\leq \# G+\omega}\right)\left(\left[\left(\exists_{<\omega}\right)\right.\right.$, $\left(\wedge_{\leq \# G+\omega}\right),\left(\operatorname{atom}\left(\Xi\left(G^{-}\right)\right)\right] \rightarrow\left(\left[\left(\exists_{<\omega}\right),\left(\wedge_{\leq \# G+\omega}\right) ;\left(\operatorname{atom}\left(\Xi\left(G^{-}\right)\right)\right]\right)\right.$tal que $\forall p^{\prime} \in U_{0}-G R A\left(G^{-}, \mathcal{E}\left(U_{0}-\right.\right.$ $S E T))$ ( $p^{\prime}$ é cone projetivo limite $\left.\Longleftrightarrow \tilde{\Xi}\left(p^{\prime}\right) \vDash \gamma_{G}^{\downarrow}\right)$.

(iii) $\forall G \in \operatorname{obj}\left(U_{0}-G R A\right) \exists \gamma_{G}^{\dagger}$ sentença em $L_{\theta_{0} \theta_{0}}\left(\Xi\left(G^{+}\right)\right)$da forma $\left(\Lambda_{\leq(\# G+\omega)}\right)\left(\forall_{<\omega}\right)\left(\left[\left(\exists_{<\omega}\right)\right.\right.$; $\left.\left(\vee_{\leq} \# G+\omega\right)\right):\left(\wedge_{<\omega}\right):\left(\right.$ atom $\left.\left(\Xi\left(G^{+}\right)\right)\right] \rightarrow\left(\left[\left(\exists_{<\omega}\right):\left(\vee_{\leq}(\# G+\omega)\right):\left(\wedge_{<\omega}\right):\left(\operatorname{atom}\left(\Xi\left(G^{+}\right)\right)\right]\right)\right.$tal que $\forall i^{\prime} \in$ $U_{0}-G R A\left(G^{+}, \mathcal{E}\left(U_{0}-S E T\right)\right)$ ( $i^{\prime}$ é cone indutivo limite $\left.\Longleftrightarrow \tilde{\Xi}\left(i^{\prime}\right) \vDash \gamma_{G}^{\uparrow}\right)$.

(i) Fixemos $X_{G_{m}^{n}}$ : variáveis em $U_{0}-S E T$ para $L_{\theta_{0} \theta_{0}}\left(\Xi\left(G_{m}^{n}\right)\right)$.

Seja $t_{n}\left(x_{-\infty}\right)$ o termo:

. $t_{n}\left(x_{-\infty}\right) m+x_{-\infty}$; caso $n=0$.

. $t_{n}\left(x_{-\infty}\right)$ m $f_{n-1}\left(f_{n-2}\left(\ldots\left(f_{1}\left(f_{0}\left(x_{-\infty}\right)\right)\right) \ldots\right)\right)$, caso $n>0$.

Seja $t_{m}^{\prime}\left(x_{-\infty}\right)$ o termo: 
. $t_{m}^{\prime}\left(x_{-\infty}\right) m x_{-x}$ : caso $m=0$.

. $t_{m}^{\prime}\left(x_{-\infty}\right) \sim m f_{m-1}^{\prime}\left(f_{m-2}^{\prime}\left(\ldots\left(f_{1}^{\prime}\left(f_{0}^{\prime}\left(x_{-\infty}\right)\right)\right) \ldots\right)\right)$, caso $m>0$.

Então a sentença $\forall x_{-\infty}\left(t_{n}\left(x_{-\infty}\right)=t_{m}^{\prime}\left(x_{-\infty}\right)\right)$ claramente satisfaz os requisitos de (i) .

(ii) Fixemos $X_{G^{-}}$, variáveis em $U_{0}-S E T$ para $L_{\theta_{0} \theta_{0}}\left(\Xi\left(G^{-}\right)\right)$.

Tendo em vista a construção canônica dos limites projetivos na categoria $U_{0}-S E T$ temos que a conjunção das sentenças abaixo satisfaz (ii):

.$\forall\left\{x_{g}\right\}_{g \in G_{0}}\left(\underset{f \in G\left(g, g^{\prime}\right)}{\wedge}\left(f\left(x_{g}\right)=x_{g^{\prime}}\right) \rightarrow \exists x_{-\infty} \underset{g \in G_{0}}{\wedge} \pi_{g}\left(x_{-\infty}\right)=x_{g}\right)$.
.$\forall\left\{x_{g}\right\}_{g \in G_{0}}\left(\exists x_{-\infty} \bigwedge_{g \in G_{0}} \pi_{g}\left(x_{-\infty}\right)=x_{g} \rightarrow \underset{f \in G\left(g, g^{\prime}\right)}{\wedge_{g}}\left(f\left(x_{g}\right)=x_{g^{\prime}}\right)\right.$.
.$\forall\left\{x_{-\infty} x_{-\infty}^{\prime}\right\}\left(\bigwedge_{g \in G_{0}}^{\wedge}\left(\left(\pi_{g}\left(x_{-\infty}\right)=\pi_{g}\left(x_{-\infty}^{\prime}\right) \rightarrow x_{-\infty}=x_{-\infty}^{\prime}\right)\right.\right.$.

(iii) Fixemos $X_{G^{+}}$: variáveis em $U_{0}-S E T$ para $L_{\theta_{0} \theta_{0}}\left(\Xi\left(G^{+}\right)\right)$.

Tendo em vista a construção canônica dos limites indutivos na categoria $U_{0}-S E T$ temos que a conjunção das sentenças abaixo satisfaz (iii):

. $\forall x_{+\infty} \underset{g \in G_{0}}{\vee} \exists x_{g}\left(\iota_{g}\left(x_{g}\right)=x_{+\infty}\right)$.

. $\hat{g}_{g, g^{\prime} \in G_{0}} \forall\left\{x_{g}, x_{g^{\prime}}^{\prime}\right\}\left(\iota_{g}\left(x_{g}\right)=\iota_{g^{\prime}}\left(x_{g^{\prime}}^{\prime}\right) \rightarrow \gamma_{g^{\prime}}^{\omega}\left(x_{g}, x_{g^{\prime}}^{\prime}\right)\right)$.

$\cdot \underset{g, g^{\prime} \in G_{0}}{\wedge} \forall\left\{x_{g}, x_{g^{\prime}}^{\prime}\right\}\left(\gamma_{g g^{\prime}}^{\omega}\left(x_{g}, x_{g^{\prime}}^{\prime}\right) \rightarrow \iota_{g}\left(x_{g}\right)=\iota_{g^{\prime}}\left(x_{g^{\prime}}^{\prime}\right)\right)$.

Onde:

- $\gamma_{g g^{\prime}}^{\omega}\left(x_{g}, x_{g^{\prime}}^{\prime}\right)$ m $\underset{n \in \omega}{\bigvee} \gamma_{g g^{\prime}}^{n+1}\left(x_{g}, x_{g^{\prime}}\right)$.

. $\gamma_{g_{g^{\prime}}}^{n+1}\left(x_{g}, x_{g^{\prime}}\right)$ ↔ $\vee\left\{\exists\left\{y_{g_{1}}^{1}, \ldots, y_{g_{n-1}}^{n-1}\right\} \wedge\left\{\gamma_{g_{0} g_{1}}^{1}\left(x_{g}, y_{g_{1}}^{1}\right), \ldots, \gamma_{g_{n-1} g_{n}}^{1}\left(y_{g_{n-1}}^{n-1}, x_{g^{\prime}}^{\prime}\right):\left\{g_{0}, g_{1}, \ldots, g_{n-1}, g_{n}\right\}\right.\right.$ $\left.\subseteq G_{0}: g=g_{0}, g_{n}=g^{\prime}\right\}$.

. $\gamma_{g^{\prime}}^{1}\left(x_{g}, x_{g^{\prime}}\right)$ ans :

.. $\vee\left\{\underset{f \in G\left(g, g^{\prime}\right)}{\vee}\left(f\left(x_{g}=x_{g^{\prime}}^{\prime}\right) ; \underset{f \in G\left(g^{\prime}, g\right)}{\vee}\left(f\left(x_{g^{\prime}}^{\prime}=x_{g}\right)\right)\right\}\right.$; para $g \neq g^{\prime}$.

..$\vee\left\{\underset{f \in G\left(g, g^{\prime}\right)}{\vee}\left(f\left(x_{g}=x_{g^{\prime}}^{\prime}\right), x_{g}=x_{g^{\prime}}^{\prime}: \underset{f \in G\left(g^{\prime}, g\right)}{\vee}\left(f\left(x_{g^{\prime}}^{\prime}=x_{g}\right)\right)\right\}\right.$ : para $g=g^{\prime}$.

Proposição 2: $\forall \mathcal{S}=(S, D, c P, c I) \in o b j\left(g n-U_{0}-E S Q\right) \exists \gamma_{\mathcal{S}}$ sentença em $L_{\theta_{0} \theta_{0}}(\Xi(S))$ da forma $\left(\wedge_{\leq \tau(\mathcal{S})}\right)\left(\forall_{<\pi(\mathcal{S})}\right)\left(\left[\left(\exists_{<\omega}\right):\left(\vee_{\leq \tau(\mathcal{S})}\right):\left(\wedge_{<\pi_{S}}\right):(\operatorname{atom}(\Xi(S))] \rightarrow\left(\left[\left(\exists_{<\omega}\right):\left(\vee_{\leq \tau(\mathcal{S})}\right):\left(\wedge_{<\pi_{\mathcal{S}}}\right)\right.\right.\right.\right.$ : (atom $(\Xi(S))])$ tal que $\forall M \in U_{0}-G R A\left(S, \mathcal{E}\left(U_{0}-S E T\right)\right.$ ) ( $M$ determina modelo de esboço $\left.\Longleftrightarrow \tilde{\Xi}(M) \vDash \gamma_{S}\right)$.

$\left.\tilde{\Xi}(M) \vDash \gamma_{S}\right)$.

- Escolhemos; para cada $G_{m}^{n} \in \mathcal{D}(\mathcal{S})$, variáveis $X_{G_{m}^{n}}$ e uma sentença $\gamma_{G_{m}^{n}}^{o}$ como na proposição (1.i) .

. Escolhemos, para cada $G \in \mathcal{P}(\mathcal{S})$, variáveis $X_{G^{-}}$e uma sentença $\gamma_{G^{-}}^{\downarrow}$ como na proposição (1.ii) .

. Escolhemos, para cada $G \in \mathcal{I}(\mathcal{S})$, variáveis $X_{G^{+}}$: uma sentença $\gamma_{G^{+}}^{\dagger}$ como na proposição (1.iii) .

. Escolhemos $X_{\mathcal{S}}$ : variáveis em $U_{0}-S E T$ para $L_{\theta_{0} \theta_{0}}(\Xi(S))$. 
. Para cada $d \in D . p \in c P . i \in c I$ escolhemos $\Psi_{d}:\left|X_{\text {dom }(d)}\right| \rightarrow\left|X_{\mathcal{S}}\right|: \Psi_{p}:\left|X_{\text {dom }(p)}\right| \rightarrow$ $\left|X_{\mathcal{S}}\right|: \Psi_{i}:\left|X_{\text {dom(i) }}\right| \rightarrow\left|X_{\mathcal{S}}\right|$ morfismos lógicos mônicos para, respectivamente, as $X_{\text {dom(d) }}{ }^{-}$

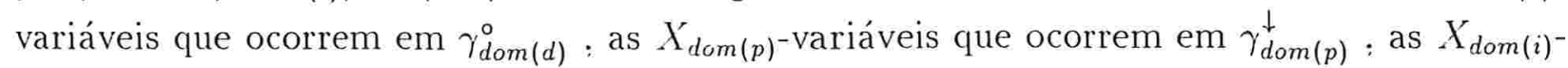
variáveis que ocorrem em $\gamma_{\text {dom(i) }}^{\uparrow}$.

Logo :

.$\dot{\Xi}(M) \vDash \hat{\Psi}_{d}\left(\gamma_{\text {dom }(d)}^{\circ}\right) \Longleftrightarrow \tilde{\Xi}(M \circ d) \vDash \gamma_{\text {dom }(d)}^{\circ}$.

. $\tilde{\Xi}(M) \vDash \hat{\Psi}_{p}\left(\gamma_{\text {dom }(p)}^{\downarrow}\right) \Longleftrightarrow \tilde{\Xi}(M \circ p) \vDash \gamma_{\text {dom }(p)}^{\downarrow}$.

. $\tilde{\Xi}(M) \vDash \hat{\Psi}_{i}\left(\gamma_{\text {dom }(i)}^{\uparrow}\right) \Longleftrightarrow \tilde{\Xi}(M \circ i) \vDash \gamma_{\text {dom(i) }}^{\uparrow}$.

Sejam:

- $\gamma_{D}=\bigwedge_{d \in D} \hat{\Psi}_{d}\left(\gamma_{\text {dom }(d)}^{\circ}\right)$.

. $\gamma_{P}=\wedge_{p \in P} \hat{\Psi}_{p}\left(\gamma_{d o m(p)}^{\downarrow}\right)$.

- $\gamma_{I}=\bigwedge_{i \in I} \hat{\Psi}_{i}\left(\gamma_{\text {dom(i) }}^{\uparrow}\right)$.

Assim, pela proposição 1, temos que $\gamma_{S}=\gamma_{D} \wedge \gamma_{P} \wedge \gamma_{I}$ satisfaz os requisitos.

Corolário : $\mathcal{L S} \downarrow$ categorial : Sejam $\mathcal{S}$ esboço natural e cardinais $\omega \leq \pi=r \epsilon g(\pi) \leq$ $\tau \leq \kappa=\left(\tau^{<\pi}\right)^{+}<\theta_{0} \operatorname{com} \pi_{\mathcal{S}} \leq \pi$ e $\tau_{\mathcal{S}} \leq \tau$ então $\forall \mathcal{M} \in \operatorname{obj}(M O D(\mathcal{S})) \forall X \in[|M|]^{<\kappa}$ $\exists \mathcal{N} \in(\operatorname{Sub}(\mathcal{M}))^{<\kappa}(X \subseteq|N|)$.

Observação 1 : Se $\mathcal{S}$ é um esboço local e $\mathcal{M} \in \operatorname{obj}(M O D(\mathcal{S})$ então $\mathcal{M}$ admite submodelos "gerados" e submodelos $<\kappa$-gerados têm cardinal $<\kappa$.

\subsection{Categorias axiomatizáveis são esboçáveis}

Proposição 1 : Sejam $\mu=\left(S, F, R, s_{F}, a_{F}, a_{R}\right) U_{0}$-tipo $\mu_{A}=\left(S, F ; \emptyset, s_{F} ; a_{F} ; \emptyset\right)$ seu tipo algébrico subjacente ; $\mu_{T}=(S, \emptyset, \emptyset, \emptyset, \emptyset, \emptyset)$ seu tipo trivial subjacente e $\gamma$ sentença em $L_{\theta_{0} \theta_{0}}(\mu)$.

(i) Se $\mu=\mu_{T}$ então $\operatorname{EST}\left(\mu, U_{0}-S E T\right)\left(\cong\left(U_{0}-S E T\right)^{\mathcal{D}(S)}\right)$ é trivialmente esboçável por $\mathcal{S}_{\mu}=\Gamma(\mu)=(S ; \emptyset, \emptyset, \emptyset)$.

(ii) Se $\mu=\mu_{A}$ então $E S T\left(\mu, U_{0}-S E T\right) \hookrightarrow\left(U_{0}-S E T\right)^{\mathcal{D}(S)}$ é projetivamente esboçável por $\mathcal{S}_{\mu_{\mathcal{T}}} \hookrightarrow \mathcal{S}_{\mu}$ onde $\mathcal{S}_{\mu}$ é um esboço especificando produtos de $\leq \sup \left\{I_{f}: f \in F\right\}$ objetos.

(iii) $E S T\left(\mu, U_{0}-S E T\right) \hookrightarrow\left(U_{0}-S E T\right)^{\mathcal{D}(S)}$ é projetivamente esboçável por $\mathcal{S}_{\mu_{T}} \hookrightarrow \mathcal{S}_{\mu}$ onde $\mathcal{S}_{\mu}$ é um esboço especificando monomorfismos ou produtos de $\leq \operatorname{máx}\left\{\sup \left\{I_{f}: f \in\right.\right.$ $\left.F\}, \sup \left\{I_{r}: r \in R\right\}\right\}$ objetos.

(iv) Se $\mu=\mu_{A}$ e $\gamma$ é da forma $(\wedge)(\forall)\left[(\wedge\right.$, atom $(\mu)]$ então $\operatorname{Mod}\left(\operatorname{atom}(\mu), \gamma, U_{0}-S E T\right) \hookrightarrow$ $\operatorname{EST}\left(\mu, U_{0}-S E T\right)$ é projetivamente esboçável por $\mathcal{S}_{\mu} \hookrightarrow \mathcal{S}_{\gamma}$ morfismo de esboços especificando produtos. 
(v) Se $\gamma$ é da forma $(\wedge)(\forall)([(\wedge), \operatorname{atom}(\mu)] \rightarrow[(\wedge)$, atom $(\mu)])$ então $\operatorname{Mod}\left(\operatorname{atom}(\mu), \gamma, U_{0}-\right.$ $S E T) \hookrightarrow E S T\left(\mu, U_{0}-S E T\right)$ é projetivamente esboçável por $\mathcal{S}_{\mu} \hookrightarrow \mathcal{S}_{\gamma}$.

(vi) Se $\gamma$ é da forma $(\wedge)(\forall)([(\wedge)$,atom $(\mu)] \rightarrow[(\exists !),(\wedge)$, atom $(\mu)])$ então $\operatorname{Mod}(\operatorname{atom}(\mu): \gamma$ : $\left.U_{0}-S E T\right) \hookrightarrow E S T\left(\mu, U_{0}-S E T\right)$ é projetivamente esboçável por $\mathcal{S}_{\mu} \hookrightarrow \mathcal{S}_{\gamma}$.

(vii) Se $\gamma$ é da forma $(\wedge)(\forall)([(\sqcup),(\wedge)$, atom $(\mu)] \rightarrow[(\sqcup),(\wedge)$, atom $(\mu)])$ então $\operatorname{Mod}(\operatorname{atom}(\mu)$, $\left.\gamma, U_{0}-S E T\right) \hookrightarrow E S T\left(\mu, U_{0}-S E T\right)$ é esboçável por $\mathcal{S}_{\mu} \hookrightarrow \mathcal{S}_{\gamma}$ onde $\mathcal{S}_{\mu}$ é esboço $\theta_{0}$-local.

(viii) Se $\gamma$ é da forma $(\wedge)(\forall)([(\exists),(\wedge)$, atom $(\mu)] \rightarrow[(\exists)$. $(\wedge)$, atom $(\mu)])$ então $\operatorname{Mod}(\operatorname{atom}(\mu)$, $\left.\gamma_{,} U_{0}-S E T\right) \hookrightarrow E S T\left(\mu, U_{0}-S E T\right)$ é esboçável por $\mathcal{S}_{\mu} \hookrightarrow \mathcal{S}_{\gamma}$ onde $\mathcal{S}_{\mu}$ é esboço $\theta_{0}$-regular.

(ix) Se $\gamma$ é da forma $(\wedge)(\forall)([(\exists),(\vee),(\wedge)$, atom $(\mu)] \rightarrow[(\exists),(\vee),(\wedge)$, atom $(\mu)])$ então $\operatorname{Mod}\left(\operatorname{atom}(\mu): \gamma, U_{0}-S E T\right) \hookrightarrow E S T\left(\mu, U_{0}-S E T\right)$ é esboçável por $\mathcal{S}_{\mu} \hookrightarrow \mathcal{S}_{\gamma}$ onde $\mathcal{S}_{\mu}$ é esboço $\theta_{0}$-geométrico.

(x) Sejam $\omega \leq \pi=r \epsilon g(\pi) \leq \tau<\theta_{0}$ onde $\pi(\mu) \leq \pi$. Se $\gamma$ é da forma $\left(\wedge_{<\tau^{+}}\right)\left(\forall_{<\pi}\right)\left(\left[\left(\exists_{<\pi}\right)\right.\right.$.

$\left.\left.\left(\vee_{<\tau^{+}}\right):\left(\wedge_{<\pi}\right), \operatorname{atom}(\mu)\right] \rightarrow\left[\left(\exists_{<\pi}\right):\left(\vee_{<\tau^{+}}\right):\left(\wedge_{<\pi}\right), \operatorname{atom}(\mu)\right]\right)$ então $\operatorname{Mod}\left(\operatorname{atom}(\mu) ; \gamma, U_{0}-\right.$ $S E T) \hookrightarrow E S T\left(\mu, U_{0}-S E T\right)$ é esboçável por $\mathcal{S}_{\mu} \hookrightarrow \mathcal{S}_{\gamma}$ onde $\mathcal{S}_{\mu}$ é um esboço $\theta_{0}$-geométrico $\operatorname{com} \pi\left(\mathcal{S}_{\mu}\right) \leq \pi$ e $\tau\left(\mathcal{S}_{\mu}\right) \leq \tau$.

(xi) Todos os morfismos de esboços acima são composições de "morfismos sobre" : todo cone proj/ind do codomínio tem base se fatorando pelo morfismo e todo objeto do codomínio ou está na imagem do morfismo ou é vértice de algum cone.

Por simples inspeção das noções de estrutura e de verdade de Tarski expressas diagramaticamente no capítulo 4 .

\subsection{Categorias esboçáveis são acessíveis}

Proposição 1 : Sejam $\mathcal{S}=(S, c P, c I) \in \operatorname{obj}\left(c-U_{0}-E S Q\right)$ e cardinais $\omega \leq \pi=$ $r \in g(\pi) \leq \tau \leq \kappa=\left((\tau)^{<\pi}\right)^{+}<\theta_{0} \operatorname{com} \pi_{\mathcal{S}} \leq \pi$ e $\tau_{\mathcal{S}} \leq \tau$ então $J_{\mathcal{S}} \in \kappa-\operatorname{ACE}\left(M O D\left(\mathcal{S}, U_{0}-\right.\right.$ $S E T)$ : $\left.\left[S ; U_{0}-S E T\right]\right)$.

$\square$ ( Rever o exemplo 3 do capítulo 2 .)

(i) $\left[S, U_{0}-S E T\right] \in \operatorname{obj}(\kappa-A C E)$.

Pois $\# S+\omega \leq \kappa$.

(ii) Pela proposição 6 do capítulo 3 temos que $J_{\mathcal{S}}: M O D(\mathcal{S}) \multimap\left[U_{0}-S E T, S\right]$ cria $U_{0}$-limites indutivos $\kappa$-filtrados.

(iii) $\{\mathcal{M} \in \operatorname{obj}(M O D(\mathcal{S})): \# M<\kappa\} \subseteq(M O D(\mathcal{S}))_{\kappa}$.

Porque $\left\{M \in \operatorname{obj}\left(\left[S, U_{0}-S E T\right]\right): \# M<\kappa\right\} \subseteq\left(\left[S, U_{0}-S E T\right]\right)_{\kappa}$ e pelo ítem (ii).

(iv) $\forall \mathcal{M} \in \operatorname{obj}(M O D(S))\left(\left(S u b(\mathcal{M})^{<\kappa}\right.\right.$; $\left.\left.\leq\right)\right)$ é conjunto ordenado $\kappa$-dirigido. 
Como $\kappa$ é regular dada $\left\{\mathcal{M}_{p}\right\}_{p \in P} \subseteq S u b(\mathcal{M})^{<\kappa} \operatorname{com} \# P<\kappa$ temos $\#\left(\cup_{p \in P}\left|M_{p}\right|\right)<\kappa$. $\operatorname{logo}$ por $\mathcal{L} \mathcal{S} \downarrow$ categorial (corolário da proposição $1 \mathrm{em}(5.1)) \exists \mathcal{N} \in\left(S u b(\mathcal{M})^{<\kappa}\left(\cup_{p \in P}\left|M_{p}\right|\right) \subseteq\right.$ $|N| \Rightarrow \forall p \in P\left(\mathcal{M}_{p} \hookrightarrow \mathcal{N}\right)$.

(v) $\forall \mathcal{M} \in \operatorname{obj}(M O D(\mathcal{S})) \forall s \in S_{0}\left(M(s)=\cup\left\{N(s): \mathcal{N} \in S u b(\mathcal{M})^{<\kappa}\right\}\right)$.

Pois, por $\mathcal{L S} \downarrow$ : $\forall x \in M(s)\left(\exists \mathcal{N} \in S u b(M)^{<\kappa}((x, s) \subseteq|N|)\right)$.

(vi) $\forall \mathcal{M} \in \operatorname{obj}\left(M O D(\mathcal{S})\right.$ ( $\mathcal{M}$ é $\mathcal{I}_{\kappa}$-limite indutivo de modelos de cardinais $<\kappa$ ) (" $\kappa$ pequenos") .

Como $I(\mathcal{M}, \kappa)$, a categoria associada a $\left(\operatorname{Sub}(\mathcal{M})^{<\kappa}\right.$ : $\left.\leq\right)$, é $\kappa$-filtrada e está praticamente em $U_{0}$ e limites indutivos em $\left[S, U_{0}-S E T\right]$ indexados por categorias (praticamente) em $U_{0}$ são pontuais, pelos ítens (ii) e (v) temos que o funtor "inclusão" $F: I(\mathcal{M}, \kappa) \mapsto M O D(\mathcal{S})$ é tal que $\underset{I(\underset{\mathcal{M}}{\longrightarrow}, \kappa)}{\lim _{(i)}} F(i)=\mathcal{M}$.

(vii) A subcategoria plena de $M O D(\mathcal{S})$ dos modelos $\kappa$-pequenos está essencialmente em $U_{0}$.

Pois a subcategoria plena de $\left[S, U_{0}-S E T\right]$ dos funtores $\kappa$-pequenos está essencialmente em $U_{0}$.

(viii) $\operatorname{plena}\left(\{\mathcal{M} \in \operatorname{obj}(M O D(\mathcal{S}): \# M<\kappa\}) \hookrightarrow M O D(\mathcal{S})\right.$ é $\mathcal{I}_{\kappa}$-ind completamento forte de categoria essencialmente em $U_{0}$.

\section{Observacao 1 :}

(i) O 2-funtor $M O D\left({ }_{-}, U_{0}-S E T\right): c-U_{0}-\mathcal{E S Q} \rightarrow U_{1}-\mathcal{C} \mathcal{A} \mathcal{T}$ se fatora por $i: \mathcal{A C E} \hookrightarrow$ $U_{1}-\mathcal{C} \mathcal{A} \mathcal{T}$.

(ii) $\mathrm{O}$ mergulho $J_{\mathcal{S}}: M O D(\mathcal{S}):\left[S, U_{0}-S E T\right]$ admite diagrama pequeno localmente livre para todo $N \in \operatorname{obj}\left(\left[S, U_{0}-S E T\right]\right.$. Em particular, temos que $\forall s \in S_{0}$ $\exists X_{s} \stackrel{Z_{s}}{\rightarrow} M O D(\mathcal{S})\left(\underset{X_{s^{*}}}{\lim } \operatorname{MOD}(\mathcal{S})\left(Z_{s}\left(x_{s}\right), \mathcal{M}\right) \cong\left[S, U_{0}-S E T\right]\left(Y_{S^{*}}(s), M\right) \cong M(s)\right.$ natural em $\mathcal{M} \in \operatorname{obj}\left(M O D(\mathcal{S})\right.$ e assim $\cup_{s \in S_{0}}\left\{M O D(\mathcal{S})\left(Z_{s}\left(x_{s}\right) ;-\right): x_{s} \in\left(X_{s}\right)_{0}\right\}$ tem cardinal pequeno e coletivamente reflete isomorfismos.

(iii) Em geral temos que se $J \in A C E\left(A, A^{\prime}\right)$ é funtor hom-bijetor e $A^{\prime}$ admite pushout (de duas flechas), então por uniformização (proposição 5 do cap.2) existe $\kappa$ tal que $J \in \kappa-A C E\left(A, A^{\prime}\right)$ e $\left(J_{0}\right)_{*}\left(A_{\kappa}\right) \subseteq A_{\kappa}^{\prime}$; tomando $i_{\kappa}: A_{\kappa} \hookrightarrow A$ temos para todo $a^{\prime} \in \operatorname{obj}\left(A_{\kappa}^{\prime}\right)$ que $\left(a^{\prime} \rightarrow J \circ i_{\kappa}\right) \simeq X_{a^{\prime}} \in \operatorname{obj}\left(U_{0}-C A T\right)$ e $X_{a^{\prime}} \cong\left(a^{\prime} \rightarrow J \circ i_{\kappa}\right) \mapsto\left(a^{\prime} \rightarrow J\right)$ é diagrama pequeno localmente livre para $J$ em $a^{\prime}$.

(iv) Pela observação (ii) acima, utilizando as proposições (9.i) e (9.ii) do ítem (1.3.1) e os desenvolvimentos no capítulo 3 temos, nas condições da proposição 1, que:

(a) $\mathcal{S}$ esboço projetivo :

. $M O D(\mathcal{S})$ admite $U_{0}$-limites indutivos $\pi$-filtrados e $U_{0}$-limites projetivos.

. $J_{\mathcal{S}}: M O D(\mathcal{S}) \mapsto\left[S, U_{0}-S E T\right]$ admite adjunto à esquerda $L$ (reflexão) : 
. $M O D(\mathcal{S})$ é $U_{0}$-ind completa.

. $\left(S^{\star} \stackrel{Z_{\mathcal{S}^{*}}}{\rightarrow} M O D(\mathcal{S})\right)=\left(S^{*} \stackrel{Y_{S^{*}}}{\rightarrow}\left[S, U_{0}-S E T\right] \stackrel{L}{\rightarrow} M O D(\mathcal{S})\right)$ é denso e $\left\{Z_{\mathcal{S}^{*}}(s): s \in S_{0}\right\} \subseteq$ $(M O D(S))_{\pi}$.

. Se $\mathcal{S}=(S, c P, \emptyset)$ é esboço categorial comutativo e se $\mathcal{S}^{\prime}$ é a $\left(\left(U_{0}-C A T\right)_{0}\right.$ : $\left.\left(U_{0}-C A T\right)_{0}\right)$ teoria associada a $M O D(\mathcal{S})$ temos que $\mathcal{S}^{\star}=\left(S^{*}, \emptyset,(c P)^{\star}\right)^{\mathcal{Z}_{\mathcal{S}^{*}}} \mathcal{S}^{\prime}$ é modelo, onde dado $(t, C)$ $\left.t: G^{\mp} \rightarrow \mathcal{E}(\mathcal{C})\right)$ cone proj/ind em uma categoria $C$ então $(t, C)^{\star}=\left(t^{\prime}, C^{\star}\right)$ é o cone ind/proj em $C^{\star}$ tal que $t^{\prime}=\left(G^{ \pm} \cong\left(G^{\mp}\right)^{\star} \stackrel{t^{\star}}{\rightarrow} \mathcal{E}\left(\mathcal{C}^{\star}\right)\right)$. Assim, pelas proposições 2 e 1 do capítulo 3 dispomos de "co-modelos canônicos" para esboços categoriais e naturais.

(b) $\mathcal{S}$ esboço local $\Rightarrow$

. $M O D(\mathcal{S})$ admite $U_{0}$-limites indutivos $\pi$-filtrados e $U_{0}$-limites projetivos conexos e não vazios.

. $J_{\mathcal{S}}: M O D(\mathcal{S}) \rightarrow\left[S, U_{0}-S E T\right]$ admite diagramas pequenos multiplamente livres (multi-reflexão).

. $M O D(\mathcal{S})$ é $U_{0}$-ind multicompleta.

. Com a notação da observação (ii) acima, tomando $\forall s \in S_{0}\left(X_{s} \cong \mathcal{D}\left(\left(X_{s}\right)_{0}\right)\right.$ temos $\cup_{s \in S_{0}}\left\{Z_{s}\left(x_{s}\right): x_{s} \in\left(X_{s}\right)_{0}\right\} \subseteq(M O D(\mathcal{S}))_{\pi}$.

\subsection{Categorias acessíveis são esboçáveis}

Para as noções bidimensionais citadas recomendamos [031] , [166] , [191] .

Consideremos as 2-categorias e 2-funtores $\mathcal{G}:\left(U_{1}-\mathcal{C} \mathcal{A} \mathcal{T}\right)^{\star} \rightleftarrows U_{1}-\mathcal{C} \mathcal{A} \mathcal{T}: \mathcal{F}$ onde $\mathcal{F}=U_{1}-\mathcal{C} \mathcal{A} \mathcal{T}\left({ }_{-}, U_{0}-S E T\right)=\mathcal{G}^{\star}$ (lembremos que (-)* "inverte" apenas as 1-flechas). Considerando para cada $U_{1}$-categoria seu funtor de avaliação obtemos uma 2-adjunção , i.e., temos transformações (estritas) entre 2-funtores $\eta: 1_{U_{1}-\mathcal{C A} \mathcal{T}} \rightarrow \mathcal{G} \circ \mathcal{F}$ e $\epsilon: \mathcal{F} \circ \mathcal{G} \rightarrow 1_{\left(U_{1}-\mathcal{C A T}\right)^{\star}}$ tais que $(\mathcal{G} \epsilon) \circ(\eta \mathcal{G})=1_{\mathcal{G}}$ e $(\epsilon \mathcal{F}) \circ(\mathcal{F} \eta)=1_{\mathcal{F}}$.

Para cada $\omega \leq \kappa=r \in g(\kappa)<\theta_{0}$, consideremos $\mathcal{P}_{\kappa}=\left\{P \in\left(U_{0}-C A T\right)_{0}: \# P<\kappa\right\}$; $\mathcal{I}_{\kappa}=\left\{I \in\left(U_{0}-C A T\right)_{0}: I\right.$ é $\mathcal{P}_{\kappa}$-filtrada $\}$ e as 2-categorias $\mathcal{B}^{\prime}{ }_{\kappa}$ e $\mathcal{A}^{\prime}{ }_{\kappa}$ que são sub-2-categorias localmente plenas de $U_{1}-\mathcal{C} \mathcal{A} \mathcal{T}$ tais que: $\operatorname{obj}\left(\mathcal{B}^{\prime}{ }^{\prime}\right)=\left\{B \in \operatorname{obj}\left(U_{1}-C A T\right): B\right.$ é $\mathcal{P}_{\kappa}$-proj completa $\wedge B$ é $\left(U_{0}-C A T\right)_{0}$-ind completa $\}$ e com morfismos os funtores que preservam $\mathcal{P}_{\kappa}$-limites projetivos e $\left(U_{0}-C A T\right)_{0}$-limites indutivos $; \operatorname{obj}\left(\mathcal{A}_{\kappa}^{\prime}\right)=\left\{A \in \operatorname{obj}\left(U_{1}-C A T\right): A\right.$ é $\mathcal{I}_{\kappa}$-ind completa $\}$ e morfismos os funtores que preservam $\mathcal{I}_{\kappa}$-limites indutivos. Utilizando a "esquizofrenia" de $U_{0}-S E T$ e a comutação de $\mathcal{I}_{k}$-limites indutivos com $\left(U_{0}-C A T\right)_{0^{-}}$ limites indutivos e $\mathcal{P}_{\kappa}$-limites projetivos em $U_{0}-S E T$, restringimos a 2-adjunção acima: $(\mathcal{F}|: \mathcal{G}|, \eta|, \epsilon|): \mathcal{B}_{\kappa}^{\prime *} \rightarrow \mathcal{A}_{\kappa}^{\prime}$.

Consideramos agora as 2-categorias $\mathcal{B}_{\kappa}$ e $\mathcal{A}_{\kappa}$ sub-2-categorias plenas e localmente plenas de, respectivamente $\mathcal{B}^{\prime}{ }_{\kappa}$ e $\mathcal{A}^{\prime}{ }_{\kappa}$, tais que $o b j\left(\mathcal{B}_{\kappa}\right)=\left\{B \in \operatorname{obj}\left(U_{1}-C A T\right): B\right.$ é $\left(U_{0}-C A T\right)_{0^{-}}$ ind completamento forte de alguma $U_{1}$-categoria essencialmente em $\left.U_{0}\right\}$ e $\operatorname{obj}\left(\mathcal{A}_{\kappa}\right)=\{A \in$ 
obj $\left(U_{1}-C A T\right): A$ é $\mathcal{I}_{\kappa}$-ind completamento forte de alguma $U_{1}$-categoria essencialmente em $\left.U_{0}\right\}$. Temos então :

(i) $\mathcal{A}_{\kappa}=\kappa-\mathcal{A C E}$.

(ii) $\operatorname{obj}\left(\mathcal{B}_{\kappa}\right)=\left\{B \in \operatorname{obj}\left(U_{1}-C A T\right): \exists C U_{1}\right.$ - categoria essencialmente em $U_{0}(B \simeq$ $\left.\left.\left[C, U_{0}-S E T\right]\right)\right\} \subseteq \operatorname{obj}\left(\mathcal{B}^{\prime}{ }_{\kappa}\right)$.

(iii) $\forall B \in \operatorname{obj}\left(\mathcal{B}_{\kappa}\right)\left(\mathcal{B}_{\kappa}^{\prime}\left(B, U_{0}-S E T\right) \simeq \mathcal{B}_{\kappa}^{\prime}\left(\left[C, U_{0}-S E T\right], U_{0}-S E T\right) \in \operatorname{obj}\left(\mathcal{A}_{\kappa}\right)\right)$

( pelo corolário da proposição 5 em (1.3.3)) .

(iv) $\forall A \in \operatorname{obj}\left(\mathcal{A}_{\kappa}\right)\left(\mathcal{A}_{\kappa}^{\prime}\left(A, U_{0}-S E T\right) \simeq\left[A_{\kappa} ; U_{0}-S E T\right] \in \operatorname{obj}\left(\mathcal{B}_{\kappa}\right)\right)$.

(v) $\left(\mathcal{B}_{\kappa}^{\prime}\left(-, U_{0}-S E T\right)\right.$ ). $\mathcal{A}_{\kappa}^{\prime}\left(-, U_{0}-S E T\right)$ I. $\eta$ I, $\left.\epsilon \mid\right): \mathcal{B}_{\kappa}^{*} \rightarrow \mathcal{A}_{\kappa}$ é biequivalência de 2-categorias;

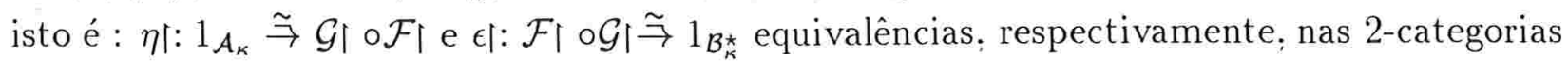
$\mathcal{H O M}\left(\mathcal{A}_{\kappa}, \mathcal{A}_{\kappa}\right)$ e $\mathcal{H O M}\left(\mathcal{B}_{\kappa}^{*}, \mathcal{B}_{\kappa}^{*}\right)$.

Em particular, (iii) nos diz que toda categoria $\kappa$-acessivel é " $\kappa$-esboçável " por um esboço grande : consideramos $\left[C, U_{0}-S E T\right]$ como uma $\left(\mathcal{P}_{\kappa}\right.$. $\left.\left(U_{0}-C A T\right)_{0}\right)$ - $U_{1}$-teoria "máxima" (i.e. com todos os cones proj/ind com essas bases). Por (v) temos $\left(\mathcal{A}_{\kappa} \stackrel{{ }^{1} \mathcal{A}_{\kappa}}{\rightarrow} \mathcal{A}_{\kappa}\right) \simeq$

$$
\left(\mathcal{A}_{\kappa} \stackrel{\dot{\mathcal{G}}}{\rightarrow} c-U_{1}-\mathcal{E S} \mathcal{Q} \stackrel{M O D\left(U_{0}-S E T\right)}{\longrightarrow} \mathcal{A}_{\kappa}\right) \text {. }
$$

Proposição 1: $\forall \omega \leq \kappa=r \epsilon g(\kappa)<\theta_{0} \forall A \in\left(U_{1}-C A T\right)_{0}(A \kappa$-acessível $\Rightarrow A$ $\kappa$-esboçável ) .

Sejam $A$ categoria $\kappa$-acessível e $C \in \operatorname{obj}\left(U_{0}-C A T\right)$ tal que $A_{\kappa} \simeq C$. Assim, basta definirmos um esboço $\mathcal{S}$ em $U_{0}$ tal que $M O D\left(\mathcal{S}, U_{0}-S E T\right) \simeq K_{\mathcal{I}_{\kappa}}(C)$.

Escolhemos $\mathcal{P}_{\kappa}^{\prime}$ esqueleto em $U_{0}-C A T$ da subcategoria plena de $U_{0}-C A T$ com objetos $\mathcal{P}_{\kappa}$. Para cada $P \in \mathcal{P}_{\kappa}^{\prime}$, e cada funtor $F: P \rightarrow C^{\star}$ escolhemos $p_{F}$ um cone projetivo limite com base $Y_{C^{\star}}$ o $F$ ( $\left[C, U_{0}-S E T\right]$ é $U_{0}$-proj completa ) e para $p_{F}$ escolhemos $i_{F}=I_{F}^{+} \rightarrow$ $\left[C, U_{0}-S E T\right]$ um cone indutivo limite com $I_{F} \in o b j\left(U_{0}-C A T\right)$ e $i_{F}(+\infty)=p_{F}(-\infty)($ $Y_{C^{\star}}$ é denso $) . \mathcal{S}=(S, c P, c I)$ é o c-esboço com categoria subjacente $S$ a subcategoria plena de $\left[C, U_{0}-S E T\right]$ com objetos os funtores $F$ descritos acima, $c P$ e $c I$ são constituídos por restrições a $S$ dos cones proj/ind descritos acima.

Temos que:

. $\mathcal{S}$ está praticamente em $U_{0}$.

. Considerando $\left[C, U_{0}-S E T\right]$ como uma $\left(\mathcal{P}_{\kappa}\right.$ : $\left.\left(U_{0}-C A T\right)_{0}\right)-U_{1}$-teoria máxima (notação

$\left.: \mathcal{C}^{k}\right)$ temos que $i: S \hookrightarrow\left[C, U_{0}-S E T\right]$ determina elemento de $c-U_{1}-E S Q\left(\mathcal{S}, \mathcal{C}_{\kappa}\right)$.

. $C^{\star} \stackrel{Y_{C^{*}}}{\rightarrow}\left[C, U_{0}-S E T\right]=C^{\star} \stackrel{Z_{k}}{\rightarrow} S \stackrel{i}{\rightarrow}\left[C, U_{0}-S E T\right]$.

Temos, a menos de identificaçòes : que :

. $\left(M O D\left(\mathcal{C}^{\kappa}, U_{0}-S E T\right)\right.$

$\left.\stackrel{\left(-\circ Y_{C^{\star}}\right) \backslash \simeq}{\longrightarrow} K_{\mathcal{I}_{\kappa}}(C) \hookrightarrow\left[C^{\star}, U_{0}-S E T\right]\right)=\left(\operatorname{MOD}\left(\mathcal{C}^{\kappa}, U_{0}-S E T\right) \stackrel{(-\circ i) \mid}{\longrightarrow} \operatorname{MOD}\left(\mathcal{S}, U_{0}-S E T\right)\right.$

$\left.\stackrel{\left(-\circ Z_{\kappa}\right) \|}{\longrightarrow}\left[C^{\star}, U_{0}-S E T\right]\right)$.

$\mathcal{S}$ satisfaz nossas expectativas: 
(i) O funtor $\left[Z_{\kappa}, U_{0}-S E T\right]:\left[S, U_{0}-S E T\right] \rightarrow\left[C^{\star}: U_{0}-S E T\right]$ se restringe a $\left(Z_{\kappa}\right)^{\prime}:$ $\operatorname{MOD}\left(\mathcal{S}, U_{0}-S E T\right) \rightarrow K_{\mathcal{I}_{\kappa}}(C)$.

Pela definição de $c P$ temos, para todo funtor $M: S \rightarrow U_{0}-S E T$ que determina modelo; que $L_{Y_{C^{*}}}\left(M \circ Z_{\kappa}\right)$ preserva $\mathcal{P}_{\kappa}$-limites projetivos. Assim a afirmação segue pela proposição 5 do ítem (1.3.3).

(ii) $\left(Z_{\kappa}\right)^{\prime}$ é hom-bijetor.

Sejam $\mathcal{M}, \mathcal{N}$ modelos de $\mathcal{S}$ e $\xi \in N A T\left(M \circ Z_{\kappa}: N \circ Z_{\kappa}\right)$. Sejam $s \in S_{0}: i_{s}: I_{s}^{+} \rightarrow S \in c I$ o cone indutivo associado a $s$ e $j_{s}: I_{s} \rightarrow C^{*}$ tal que $\left.i_{s}\right|_{I_{s}}=Z_{\kappa} \circ j_{s}$. Como $M, N$ determinam modelos definimos $M(s) \stackrel{\xi_{s}^{\prime}}{\rightarrow} N(s)=\underset{I_{s}}{\lim } M \circ Z_{\kappa} \circ j_{s} \stackrel{\stackrel{\lim \xi_{j s}}{\longrightarrow}}{\rightarrow} \underset{I_{s}}{\lim } N \circ Z_{\kappa} \circ j_{s}$.

Pela escolha de $c I$ temos $\left(\xi_{s}^{\prime}\right)_{s \in S_{0}}$ é transformação natural. Além disso $\xi^{\prime}$ é a única transformação natural tal que $\xi_{Z_{\kappa}}^{\prime}=\xi$.

(iii) $K_{\mathcal{I}_{\kappa}}(C)=\left[\left(\left(Z_{\kappa}\right)_{0}^{\prime}\right)_{*}\left(\operatorname{MOD}\left(\mathcal{S}, U_{0}-S E T\right)\right)\right]^{\cong}$.

Pois para $F \in \operatorname{obj}\left(K_{\mathcal{I}_{\kappa}}(C)\right)$ temos $\left.F \cong\left(L_{Y_{C \star}}(F)\right) \circ Y_{C^{\star}} \cong\left(\left(L_{Y_{C \star}}(F)\right) \circ i\right) \circ Z_{\kappa}\right)$

Observação 1 : Pelas considerações no início deste ítem, pelo princípio da uniformização (proposição 5 do cap.2) e tomando por base a construção na proposição 1 acima obtemos o "teorema que esboçabilidade uniforme" (teo. 4.4.1 em [166]) : $\forall \mathcal{X}$ 2-categoria em $U_{0}$ $\forall \Gamma: \mathcal{X} \rightarrow \mathcal{A C E}$ homomorfismo de 2-categorias $\exists \Gamma^{\prime}: \mathcal{X} \rightarrow\left(c-U_{0}-\mathcal{E S Q}\right)^{\star}$ homomorfismo de 2-categorias tal que temos equivalência (na 2-categoria $\mathcal{H O M}(\mathcal{X}, \mathcal{A C E}))(\mathcal{X} \stackrel{\Gamma}{\rightarrow} \mathcal{A C E}$ ) $\simeq\left(\mathcal{X} \stackrel{\Gamma^{\prime}}{\rightarrow}\left(c-U_{0}-\mathcal{E S Q}\right)^{\star} \stackrel{M O D\left(U_{0}-S E T\right)}{\longrightarrow} \mathcal{A C E}\right)$.

\subsection{Considerações adicionais}

(1)

Pelas observação 8 e proposição 3 do capítulo 4 e pelos ítens (5.2) e (5.3) temos que para todo $U_{0}$-tipo $\mu$, toda $U_{0}$-classe dos $U_{0}-S E T$ - modelos de alguma sentença em $L_{\theta_{0} \theta_{0}}(\mu) \mathrm{mu}$ nida de uma noção de morfismo suficientemente restritiva, constitui uma categoria acessível.

(2)

Pela observação 1 em (5.4) todo funtor acessível é esboçável. Assim pela observação 1 do ítem (5.3) e a parte externa do diagrama abaixo : temos que todo funtor acessível admite diagrama pequeno localmente livre ( de "tipo" associado apenas a seu domínio) . 


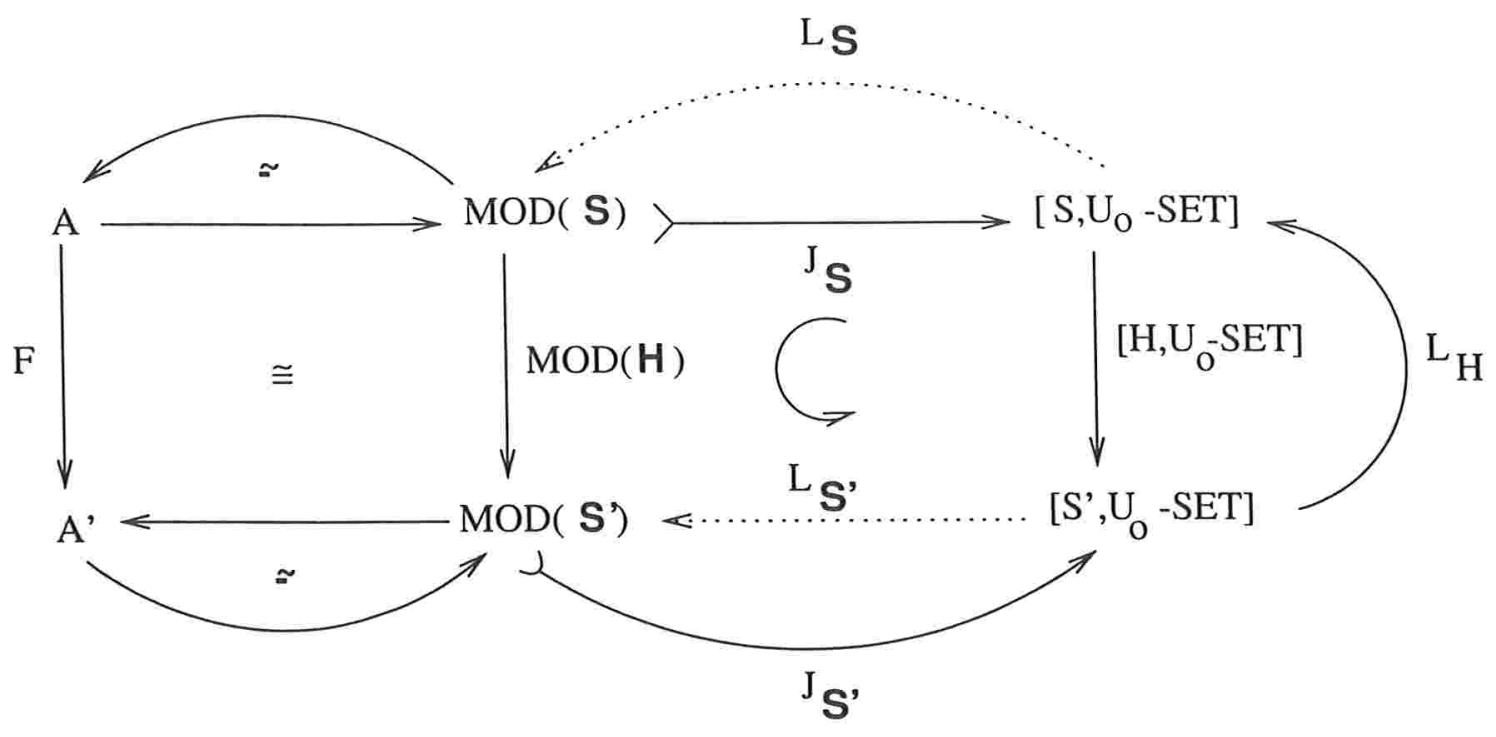

(3)

Em (5.4) vimos que categorias $\kappa$-acessíveis são $\kappa$-esboçáveis e em (5.3) que categorias $\pi$ esboçáveis são $\kappa$-acessiveis para algum $\kappa \geq \pi$. Abaixo apresentaremos subcategorias plenas de $A C E$ e $c-U_{0}-E S Q$ onde $\kappa$-acessíbilidade equivale a $\kappa$-esboçabilidade.

Proposição 1 : Sejam $\omega \leq \pi=r \epsilon g(\pi)<\theta_{0}$ e $A \in \operatorname{obj}\left(U_{1}-C A T\right)$. São equivalentes :

(i) $A$ é $\pi$-esboçável por $\pi$-esboço projetivo.

(ii) $A$ é $\pi$-acessível e $U_{0}$-proj completa.

(iii) $A$ é $\pi$-acessivel e $U_{0}$-ind completa.

(iv) $A$ é $\pi$-acessível e $A_{\pi}$ é $\mathcal{P}_{\pi}$-ind completa, onde $\mathcal{P}_{\pi}=\left\{P \in \operatorname{obj}\left(U_{0}-C A T\right): \# P<\pi\right\}$.

(v) $\exists C$ ( $C$ é $U_{1}$-categoria essencialmente em $U_{0} \wedge C$ é $\mathcal{P}_{\pi}$-ind completa $\wedge A \simeq\left[C^{\star} ; U_{0}-\right.$

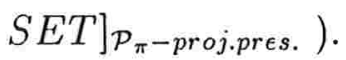

(vi) $A$ é $\pi$-localmente presentável. (consultar [092])

$((\mathrm{i}) \Rightarrow(\mathrm{ii})) \wedge((\mathrm{i}) \Rightarrow(\mathrm{iii}))$

Pela observação (1.iv.a) em (5.3) basta verificar que $A \pi$-projetivament esboçável $\Rightarrow A$ $\pi$-acessível .

Seja $\mathcal{S} \pi$-esboco categorial projetivo tal que $\exists E: M O D(\mathcal{S}) \stackrel{\simeq}{\rightarrow}$. Nas condições da observação (1.iv.a) em (5.3) denotamos $C$ a subcategoria plena de $M O D(\mathcal{S})$ com $C_{0}=$ $\left[\left\{Z_{\mathcal{S}^{*}}(s): s \in S_{0}\right\}\right]^{\mathcal{P}_{\pi}-l i m} \rightarrow$ Seja $(C \stackrel{J}{\rightarrow} A)=(C \hookrightarrow M O D(\mathcal{S}) \stackrel{\sim E}{\rightarrow} A)$. Utilizando as observações (1.ii) e (1.iv.a) em (5.3) temos:

. $\left\{M O D(\mathcal{S})(c,-): c \in C_{0}\right\}$ reflete isomorfismos.

- Pela construção transfinita de $C$ temos:

.. $C \hookrightarrow(M O D(S))_{\pi}$. 
.. $C$ é $U_{1}$-categoria essencialmente em $U_{0}$.

. $\forall a \in A_{0}\left((J \rightarrow a)\right.$ é $U_{1}$-categoria essencialmente em $U_{0} \wedge(J \rightarrow a)$ é $\mathcal{P}_{\pi}$-ind completa ) . $\left.\left.J) \stackrel{\cong\left(J(c) \cdot \operatorname{can}_{a}\right)}{\longrightarrow} A(J(c), a)\right)\right)$.

(ii) $\Rightarrow$ (iii)

Pois $\forall I\left(I \in \operatorname{obj}\left(U_{0}-C A T\right) \Rightarrow\left(\Delta_{I} \in A C E(A,[I, A])\right.\right.$ admite diagrama pequeno localmente livre e preserva $U_{0}$-limites projetivos logo, pela proposição (9.i) em (1.3.1), $\Delta_{I}$ admite adjunto à esquerda ) ) .

(iii) $\Rightarrow$ (iv) Pelas proposição 4 em (1.3.2) e observação (1.iii) em (1.3.3) .

(iv) $\Rightarrow$ (i)

Seja $C \in \operatorname{obj}\left(U_{0}-C A T\right)\left(\left(A_{\pi}\right) \simeq C\right)$ então $C$ é $\mathcal{P}_{\pi}$-ind completa : $\left(\mathcal{P}_{\pi}\right)^{*}=\mathcal{P}_{\pi}$ e $\forall P \in \mathcal{P}_{\pi}$ $\forall \Gamma: P \rightarrow C^{\star}\left(\underset{P}{\lim } Y_{C^{\star}} \circ \Gamma(p) \cong\left[P^{\star}, C\right]\left(\Gamma^{\star}, \Delta_{P^{\star}}(-)\right) \cong Y_{C^{\star}}\left(\underset{P^{\star}}{\lim } \Gamma^{\star}(p)\right) \cong \underset{I^{\star}}{\lim } Y_{C^{\star}}\left(\underset{P^{\star}}{\lim } \Gamma^{\star}(p)\right)\right.$ onde $I \cong 1$.

Assim, o resultado segue pela construção na proposição 1 em (5.4) e porque cones indutivos 1-indexados simplesmente especificam isomorfismos.

(iv) $\Longleftrightarrow$ (v)

Pelos ítens 1,2,4,5 da proposição 5 em (1.3.3) .

(v)

Contido na referência [092].

Corolário : $\forall \omega \leq \kappa \leq \kappa^{\prime}<\theta_{0}\left(A \kappa\right.$-localmente presentável $\Rightarrow A \kappa^{\prime}$-localmente presentável ).

Pois (vi) $\Rightarrow(\mathrm{i}) \Rightarrow($ vi) .

Proposição 2: Sejam $\omega \leq \pi=r \epsilon g(\pi)<\theta_{0}$ e $A \in \operatorname{obj}\left(U_{1}-C A T\right)$. São equivalentes :

(i) $A$ é $\pi$-esboçável por $\pi$-esboço local.

(ii) $A$ é $\pi$-acessível e admite $U_{0}$-limites projetivos conexos e não vazios.

(iii) $A$ é $\pi$-acessível e $U_{0}$-ind multicompleta.

(iv) $A$ é $\pi$-acessivel e $A_{\pi}$ é $\mathcal{P}_{\pi}$-ind multicompleta, onde $\mathcal{P}_{\pi}=\left\{P \in \operatorname{obj}\left(U_{0}-C A T\right)\right.$ : $\# P<\pi\}$.

(v) $\exists C$ ( $C$ é $U_{1}$-categoria essencialmente em $U_{0} \wedge C$ é $\mathcal{P}_{\pi}$-ind multicompleta $\wedge A \simeq$ $\left.\left[C^{\star}, U_{0}-S E T\right]_{m u l t i-\mathcal{P}_{\pi}-\text { proj.fund } \epsilon}\right)$.

(vi) $A$ é $\pi$-localmente multipresentável. (consultar [067])

(vii) $A$ é $\pi$-localizável. (consultar [063]) 
((i) $\Rightarrow($ ii) $) \wedge(($ i $\Rightarrow$ (iii)) Pela observação (1.iv.b) em (5.3) basta verificar que $A$ esboçável por $\pi$-esboço local $\Rightarrow A \pi$-acessível .

Seja $\mathcal{S} \pi$-esboco categorial local tal que $\exists E: M O D(\mathcal{S}) \cong A$. Nas condições da observação (1.iv.b) em (5.3) denotamos $C$ a subcategoria plena de $M O D(\mathcal{S}) \operatorname{com} C_{0}=\left[\left\{Z_{s}\left(x_{s}\right)\right.\right.$ : $\left.\left.s \in S_{0} \wedge x_{s} \in\left(X_{s}\right)_{0}\right\}\right]^{\mathcal{P}_{\pi}-\text { multilim }} \stackrel{\text { Seja }}{\longrightarrow}(C \stackrel{J}{\rightarrow} A)=(C \hookrightarrow M O D(\mathcal{S}) \stackrel{\simeq E}{\rightarrow} A)$. Utilizando as observações (1.ii) e (1.iv.b) em (5.3) temos:

. $\left\{M O D(\mathcal{S})(c,-): c \in C_{0}\right\}$ reflete isomorfismos.

. Pela construção transfinita de $C$ temos:

.. $C \hookrightarrow(M O D(\mathcal{S}))_{\pi}$.

.. $C$ é $U_{1}$-categoria essencialmente em $U_{0}$.

. $\forall a \in A_{0}\left((J \rightarrow a)\right.$ é $U_{1}$-categoria essencialmente em $U_{0} \wedge(J \rightarrow a)$ é $\mathcal{P}_{\pi}$-ind completa ).

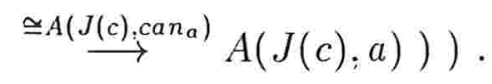

. $J$ é denso pois $\forall a \in A_{0}\left(\exists \underset{J \rightarrow a}{\lim } P(a) \circ J \stackrel{c a n a}{\rightarrow} a \wedge \forall c \in C_{0}(A(J(c), \underset{J \rightarrow a}{\lim } P(a) \circ J)\right.$ (ii) $\Rightarrow$ (iii)

Pois $\forall I\left(I \in \operatorname{obj}\left(U_{0}-C A T\right) \Rightarrow\left(\Delta_{I} \in A C E(A .[I, A])\right.\right.$ admite diagrama pequeno localmente livre e preserva $U_{0}$-limites projetivos conexos e não vazios logo, pela proposição (9.ii) em (1.3.1), $\Delta_{I}$ admite multiadjunto à esquerda ) ) .

(iii) $\Rightarrow$ (iv)

Pelas proposição 4 em (1.3.2) e observação (1.iii) em (1.3.3) .

(iv) $\Rightarrow$ (i) Seja $C \in \operatorname{Obj}\left(U_{0}-C A T\right)\left(\left(A_{\pi}\right) \simeq C\right)$ então $C$ é $\mathcal{P}_{\pi}$-ind multicompleta ; $\left(\mathcal{P}_{\pi}\right)^{\star}=\mathcal{P}_{\pi}$ e $\forall P \in \mathcal{P}_{\pi} \forall \Gamma: P \rightarrow C^{\star}\left(\underset{P}{\lim } Y_{C^{\star}} \mathrm{o} \Gamma(p) \cong\left[P^{\star}: C\right]\left(\Gamma^{\star}, \Delta_{P^{\star}}(-)\right) \cong \underset{I^{\star}}{\lim } Y_{C^{\star}}\left(\left(\Gamma^{\prime}\right)^{\star}(i)\right)\right.$ onde $I \cong \mathcal{D}\left(I_{0}\right)$.

Assim, o resultado segue pela construção na proposição 1 em (5.6) .

(iv) $\Longleftrightarrow(\mathrm{v})$

Pelos ítens 1,2,4,6 da proposição 5 em (1.3.3) .

(v) $\Longleftrightarrow(\mathrm{vi})$

Contido na referência [067].

(vi) $\Longleftrightarrow$ (vii)

Mesmo conceito, nomenclaturas distintas ( [067] ; [063] ).

Corolário : $\forall \omega \leq \kappa \leq \kappa^{\prime}<\theta_{0}$ ( $A \kappa$-localmente multipresentável $\Rightarrow A \kappa^{\prime}$-localmente multipresentável ) .

Pois (vi) $\Rightarrow(\mathrm{i}) \Rightarrow($ vi) . 
Observação 1 : Caracterizações análogas às das proposições 1 e 2 acima são demonstrados em [013] e [007]. 


\section{Capítulo 6}

\section{Miscelânea}

Neste capítulo apresentaremos alguns exemplos e contra-exemplos de categorias acessíveis utililizando as caracterizações no capítulo anterior, mencionando simultaneamente outros resultados interessantes ${ }^{1}$.

(1)

Variedades de álgebras são $\omega$-localmente presentáveis.

(i) Pelo teorema de Birkhoff, variedades são categorias de modelos de teorias equacionais em $L_{\omega \omega}(\mu)$ para algum tipo $\mu$ unisortido, sem símbolos relacionais, e com símbolos funcionais com aridades finitas.

(ii) Se $A \stackrel{i}{\hookrightarrow} \operatorname{EST}(\mu)$ é variedade, então $i$ é projetivamente esboçável por morfismo de esboços que especificam produtos finitos.

(iii) Se $L: S E T \rightarrow A$ denota o adjunto à esquerda de $E: A \stackrel{i}{\hookrightarrow} \operatorname{EST}(\mu) \rightarrow[\mathcal{D}(S), S E T] \cong$ $[1, S E T] \cong S E T: a \in A_{0}$ e $\omega \leq \kappa=r \in g(\kappa)<\infty$ então:

(iii.a) São equivalentes:

. $\exists X \in[E(a)]^{<\kappa} a=\bigvee\{b: b \hookrightarrow a \wedge X \subseteq E(b)\}$ (" $a$ é $<\kappa$-gerado por $X "$ ) .

. $\exists X \in V \exists f: L(X) \rightarrow a(\# X<\kappa \wedge f$ é epimorfismo extremal (i.e. $f$ não se fatora por subojeto próprio de $a)$ ) .

. $\forall I \stackrel{F}{\rightarrow} A=I \stackrel{F^{\prime}}{\rightarrow} \operatorname{mono}(A) \hookrightarrow A(I$ é categoria pequena $\kappa$-filtrada $\Rightarrow \underset{I}{\lim } A(a, F(i))$ $\stackrel{\operatorname{can} \cong}{\longrightarrow} A(a, \underset{I}{\lim } F(i)))$ ("a é $\kappa$-gerável" [092]).

(iii.b) São equivalentes:

. $\exists(X, R) \in V\left(R \subseteq E(L(X)) \times E(L(X)) \wedge R^{\prime}=\bigvee\{C: C\right.$ é congruência em $L(X) \wedge$ $R \subseteq C\} \wedge a \cong L(X) / R^{\prime} \wedge \# X<\kappa \wedge \# R<\kappa$ ) ("a é $<\kappa$-presentado por $(X, R)$ ") .

. $\exists(X, R) \in V \exists L(R) \rightrightarrows L(X) \stackrel{f}{\rightarrow} a$ coigualador $(\# X<\kappa \wedge \# R<\kappa)$.

\footnotetext{
${ }^{1}$ Utilizaremos a "fundamentação dos universos" somente quando esta for indispensável.
} 


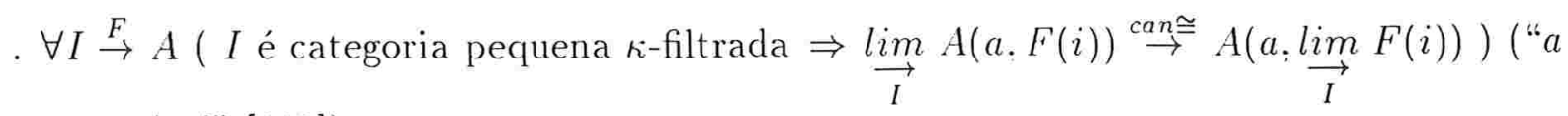
é $\kappa$-presentável" [092]) .

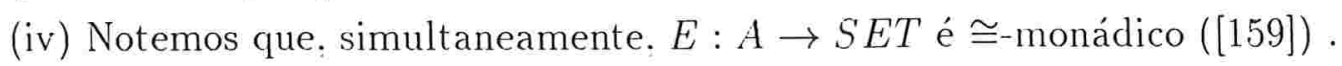

Revendo a seção (1.2.1) percebemos que $\mathcal{E}: C A T \rightarrow G R A$ é esboçável por morfismo de esboços $\mathcal{S}_{C A T} \hookrightarrow \mathcal{S}_{G R A}$ onde $\mathcal{S}_{G R A}$ é um $\omega$-esboço trivial e $\mathcal{S}_{C A T}$ é um $\omega$-esboço projetivo.

(3) [094]

Sejam:

. $\omega \leq \kappa=r \in g(\kappa)<\theta_{0}$.

- $(L, i): U_{1}-$ pré $O R D \rightarrow U_{1}-O R D$ a reflexão da categoria dos conjuntos ordenados pertencentes a $U_{1}$ e funções crescentes na categoria dos conjuntos pré-ordenados pertencentes a $U_{1}$ e funções crescentes.

- $U_{1}-R E a l_{\kappa}$ a categoria dos $\kappa$-reticulados algébricos pertencentes a $U_{1}$, com a subclasse dos $\kappa$-compactos com cardinais $<\theta_{0}$ e funções $\kappa$-Scott-contínuas.

. $\kappa-L O C P R E S$ a subcategoria plena de $\kappa-A C E$ das categorias $\kappa$-localmente presentáveis.

Então a "retração canônica" : ( $U_{1}-O R D \hookrightarrow U_{1}-\operatorname{préORD} \rightarrow U_{1}-C A T \rightarrow U_{1}-$ $\left.\operatorname{pré} O R D \stackrel{L}{\rightarrow} U_{1}-O R D\right) \cong\left(U_{1}-O R D \stackrel{\text { id }}{\rightarrow} U_{1}-O R D\right)$ se restringe à $\left(U_{1}-R E a l_{k} \mapsto\right.$ $\left.\kappa-L O C P R E S \rightarrow U_{1}-R E a l_{\kappa}\right) \cong\left(U_{1}-R E a l_{\kappa} \stackrel{\text { id }}{\rightarrow} U_{1}-R E a l_{\kappa}\right)$.

(4)

Considerando sua axiomatização usual notamos que ORD : (ORDtot ), a categoria dos conjuntos ordenados (totalmente ordenados) e funções crescentes (estritamente crescentes) é $\omega$-localmente presentável ( $\omega$-localmente multipresentável) .

Do mesmo modo notamos que $B O O(\sigma-B O O)$; a categoria das álgebras booleanas ( $\sigma$-álgebras booleanas) e homomorfismos ( $\sigma$ - homomorfismos) é $\aleph_{0}$-localmente presentável ( $\aleph_{1}$ - localmente presentável) .

(5) $[186]$

Seja $A$ topos de Grothendieck e $\mathcal{C}=(C, \mathcal{J})$ um sítio pequeno com $A \simeq S H(\mathcal{C})$. Como $S H(\mathcal{C}) \hookrightarrow P S H(\mathcal{C})=\left[C^{\star}: S E T\right]$ é inclusão plena com $\operatorname{obj}(S H(\mathcal{C}))=\left\{F: C^{*} \rightarrow S E T:\right.$ $\left.\forall c \in C_{0} \forall R \stackrel{i_{R}}{\leftrightarrow} C(\ldots, c) \in \mathcal{J}(c)\left(N A T(R, F) \stackrel{\left(-i_{R}\right) \cong}{\leftarrow} N A T(C(\ldots, c), F)\right)\right\}=\left\{F: C^{\star} \rightarrow S E T\right.$

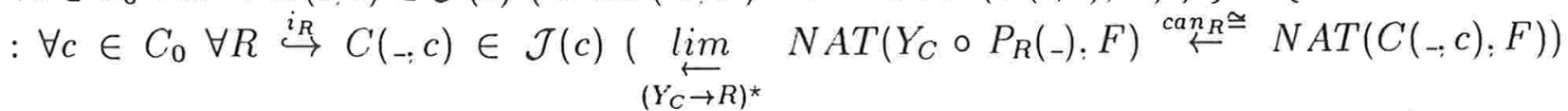
\}$=\left\{F: C^{\star} \rightarrow S E T: \forall c \in C_{0} \forall R \stackrel{i_{R}}{\rightarrow} C(-, c) \in \mathcal{J}(c)\left(\underset{K(R)}{\lim } N A T\left(Y_{C} \circ P_{R}(-), F\right) \stackrel{c a n_{R}^{\prime} \simeq}{\leftarrow} F(c)\right)\right.$ \} temos que $S H(\mathcal{C}) \cong M O D\left(\mathcal{S}^{\star}\right)$ onde $\mathcal{S}$ é o cc-esboço indutivo sobre $C$ com cones indutivos 
canônicos associados a $\left(Y_{C} \rightarrow R\right) \stackrel{P(R)}{\rightarrow} C, R \in \cup_{c \in C_{0}} \mathcal{J}(c)$. Portanto toposes de Grothendieck são categorias localmente presentáveis.

(6) $[051]:[013]$

Sejam $\mathcal{P}: \mathcal{I} \in\left[\operatorname{obj}\left(U_{0}-G R A\right)\right]^{<\theta_{0}}$. Então $c c-(\mathcal{P} . \mathcal{I})-U_{0}-E S Q \stackrel{\mathcal{E}}{\rightarrow} U_{0}-C A T$ e $(\mathcal{P} . \mathcal{I})-$ $U_{0}-T Y P \rightarrow c c-(\mathcal{P}: \mathcal{I})-U_{0}-E S Q$ são projetivamente esboçáveis e $(\mathcal{P}: \mathcal{I})-U_{0}-T E O$ é esboçável por esboço regular.

(7) $[063],[066]:[067]$

(i) Consideremos as subcategorias da categoria $\omega$-localmente presentável $A u c$ dos anéis com unidade e comutativos, cujos objetos estão entre os anéis não triviais $(0 \neq 1)$ e os morfismos são mergulhos ( refletem a relação $i g(x, y): x=y$ )

- $A u c^{\#} \hookrightarrow A u c$ : a categoria dos anéis com unidade, comutativos e não triviais com mergulhos entre esses é subcategoria multireflexiva de $A u c$ :

. $.0 \cong A \in \operatorname{obj}(A u c) \mapsto\{0 \stackrel{!}{\rightarrow} \mathbb{Z}\} \cup\{0 \stackrel{!}{\rightarrow} \mathbb{Z} / n \mathbb{Z}: n \in \mathbb{Z} . n>0\}$.

.. $0 \supsetneqq A \in \operatorname{obj}(A u c) \mapsto\left\{A \stackrel{\pi_{I}}{\rightarrow} A / I: I\right.$ é ideal próprio de $\left.A\right\}$.

. $D u c^{\#} \hookrightarrow A u c^{\#} \hookrightarrow A u c$, a categoria dos domínios de integridade com unidade, comutativos e não triviais com mergulhos entre esses é subcategoria multireflexiva de $A u c$ :

. $.0 \cong A \in \operatorname{obj}(A u c) \mapsto\{0 \stackrel{!}{\rightarrow} \mathbb{Z}\} \cup\{0 \stackrel{!}{\rightarrow} \mathbb{Z} / p \mathbb{Z}: p \in \mathbb{Z}: p$ é primo $\}$.

.. $0 \supsetneqq A \in \operatorname{obj}(A u c) \mapsto\left\{A \stackrel{\pi_{P}}{\rightarrow} A / P: P\right.$ é ideal primo próprio de $\left.A\right\}$.

Essas categorias são categorias $\omega$-localmente multipresentáveis (e $\omega$-multialgébricas) que não são localmente presentáveis pois, sendo desconexas (homomorfismos injetivos preservam característica), não admitem produtos e coprodutos.

(ii) Consideremos as subcategorias da categoria $\omega$-localmente presentável $A u c$ dos anéis com unidade e comutativos, cujos objetos estão entre os anéis não triviais $(0 \neq 1)$ e os morfismos são locais ( refletem a relação $i n v(x): \exists y(x . y=1)$ ) .

- $A u c^{\varangle} \hookrightarrow A u c$, a categoria dos anéis com unidade, comutativos e não triviais (com homomorfismos locais entre esses) é subcategoria multireflexiva de $A u c$ :

. $.0 \cong A \in \operatorname{obj}(A u c) \mapsto\{\mathbf{0} \stackrel{!}{\rightarrow} \mathbb{Q}\} \cup\left\{\mathbf{0} \stackrel{!}{\rightarrow} \mathbb{Z}_{p \mathbb{Z}}: p \in \mathbb{Z}: p\right.$ é primo $\}$.

.. $0 ¥ A \in \operatorname{obj}(A u c) \mapsto\left\{A \stackrel{\pi_{S}}{\rightarrow} A\left[S^{-1}\right]: S\right.$ é submonóide multiplicativo , disjunto de $\{0\}$ de $A\}$.

. $L u c^{\varangle} \hookrightarrow A u c^{\varangle} \hookrightarrow A u c$ a categoria dos anéis locais com unidade, comutativos e não triviais (com homomorfismos locais entre esses) é subcategoria multireflexiva de $A u c$ :

.. $0 \cong A \in \operatorname{obj}(A u c) \mapsto\{0 \stackrel{!}{\rightarrow} \mathbb{Q}\} \cup\left\{0 \stackrel{!}{\rightarrow} \mathbb{Z}_{p \mathbb{Z}}: p \in \mathbb{Z} . p\right.$ é primo $\}$.

.. $0 \not A \in \operatorname{obj}(A u c) \mapsto\left\{A \stackrel{\pi_{P}}{\rightarrow} A_{P}: P \in \operatorname{Sp} \in c(A)\right\}$.

Essas categorias são categorias $\omega$-localmente multipresentáveis (e $\omega$-multialgébricas) que não são localmente presentáveis pois, sendo desconexas (homomorfismos locais preservam a 
"característica de inversibilidade" : $\left.\operatorname{car}_{\text {inv }}(A)=\min \{n \in \mathbb{N}: A \not k i n v(n .1)\}\right)$, não admitem produtos e coprodutos.

(iii) Seja $K c$, a categoria dos corpos comutativos:

- Kic é axiomatizável pelos axiomas usuais para anéis com unidade e comutativos e o axioma $\forall x(x=0 \sqcup \exists y(x . y=1))$.

$K c$ satisfaz as condições em (i) e (ii) acima e $K c \hookrightarrow A u c$ se fatora como $K c \hookrightarrow D u c^{\#} \hookrightarrow$ $A u c \# \hookrightarrow A u c$ e $K c \hookrightarrow A u c^{\triangleleft} \hookrightarrow A u c$.

. $K c \hookrightarrow D u c^{\#}$ admite adjunto à esquerda : o funtor "corpo das frações" : assim $K c \hookrightarrow A u c$ admite multiadjunto a esquerda:

.. $0 \cong A \in \operatorname{obj}(A u c) \mapsto\{0 \stackrel{!}{\rightarrow} \mathbb{Q}\} \cup\{0 \stackrel{!}{\rightarrow} \mathbb{Z} / p \mathbb{Z}: p \in \mathbb{Z}: p$ é primo $\}$.

.. $0 \supsetneqq A \in \operatorname{obj}(A u c) \mapsto\left\{A \stackrel{\pi_{P}}{\rightarrow} A / P \stackrel{\imath P}{\rightarrow} \operatorname{Frac}(A / P): P \in \operatorname{Spec}(A)\right\}$.

(8)

- As categorias $M \epsilon t$ : Norm e Euc, respectivamente; dos espaços métricos e imersões isométricas , dos espaços normados e imersões isométricas e dos espaços euclidianos e transformações lineares ortogonais são $\aleph_{0}$-multialgébricas e portanto $\aleph_{0^{-}}$multipresentáveis. ([066]:

[067].:[063])

- As categorias $M \epsilon t c, B a n$ e Hilb, respectivamente, dos espaços métricos completos e imersões isométricas, dos espaços de Banach e imersões isométricas e dos espaços de Hilbert e transformações lineares ortogonais são $\aleph_{1}$-multialgébricas, portanto $\aleph_{1}$ - multipresentáveis. ([066];[067]:[063])

- A categoria $B a n_{\leq}$dos espaços de Banach e contrações é axiomatizável por sentença em $L_{\aleph_{1} \aleph_{1}}(\mu)$ da forma $(\Lambda)(\forall)([(\vee),(\wedge)$ : $(\operatorname{atom}(\mu))] \rightarrow[(\exists),(\vee),(\wedge),($ atom $(\mu))])$ onde $\mu$ é um tipo unisortido com $\omega$ símbolos funcionais e um símbolo relacional. ([166])

- A categoria $\mathbb{C}^{*}-A L G u c$ das $\mathbb{C}^{*}$-álgebras unitárias e comutativas é $\aleph_{1}$-localmente presentável. ([092])

Temos alguns contra-exemplos nos ítens (9),(10),(11) abaixo.

- $(S E T)^{\star}$ é categoria proj/ind completa não acessível . ([092]:[090])

Pois $\cup_{\kappa<\infty}\left((S E T)^{*}\right)_{\kappa}=\{X \in V: \# X=1\} \subsetneq \operatorname{obj}\left((S E T)^{\star}\right)$.

Em geral, devido ao:

Teorema ([092]) : Se $A$ e $A^{\star}$ são categorias localmente presentáveis então $A$ (e $\left.A^{*}\right)$ são pré-reticulados completos.

Obtemos o seguinte:

"Princípio de não dualidade" : Seja $A$ categoria acessível e proj completa e/ou ind completa. Se $A$ não é categoria pré ordenada então $A^{\star}$ é categoria proj-ind completa não acessivel . 
Assim :

- CHaus a categoria dos espaços compactos e $T_{2}$ é categoria proj/ind completa não acessível . ([092])

Pois $(\text { CHaus })^{*} \simeq \mathbb{C}^{*}-A L G u c$ (dualidade de Gelfand).

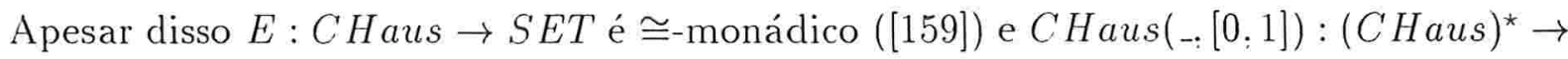
$S E T$ é $\simeq$-monádico $([026])$.

- CHaus0D a categoria dos espaços compactos, $T_{2}$ com base de clopens é categoria $\operatorname{proj} /$ ind completa não acessível .

Pois $(C H a u s 0 D)^{\star} \simeq B O O$ (dualidade de Stone) .

. Como $(H i l b)^{\star} \cong H i l b$ vemos que Hilb é categoria localmente multipresentável não localmente presentável.

- TOP é categoria proj/ind completa, tal que $E: T O P \rightarrow S E T$ admite adjunto à esquerda e à direita mas:

.. TOP não é acessível. ([092]:[090])

Pois $\cup_{\kappa<\infty}(T O P)_{\kappa}=\{(X, \tau) \in \operatorname{obj}(T O P): \tau=\mathcal{P}(x)\} \subsetneq \operatorname{obj}(T O P)$.

.. $E: T O P \rightarrow S E T$ não é $\simeq$-monádico. $([026])$

Pois $E$ não reflete isomorfismos.

.. Apesar disso, em [181] demonstra-se que TOP é equivalente a categoria de modelos de uma sentença $(\Lambda)(\forall)([(\wedge)(\operatorname{atom}(\mu))] \rightarrow[\operatorname{atom}(\mu)])$ onde $\mu$ é sobre um tipo "grande" .

. $(T O P)^{\star}$ é categoria proj/ind completa mas:

.. $(T O P)^{\star}$ não é acessível. ([092],[090])

Pois $\cup_{\kappa<\infty}\left((T O P)^{\star}\right)_{\kappa}=\{(X, \tau) \in o b j(T O P): \# X=1\} \subsetneq o b j\left((T O P)^{\star}\right)$.

.. Contudo, em [025] demonstra-se que $(T O P)^{\star}$ é equivalente a categoria de modelos, sobre uma categoria $\simeq$-monádica sobre $S E T$; de uma sentença $(\wedge)(\forall)([(\wedge)$, $(\operatorname{atom}(\mu))] \rightarrow$ $[\operatorname{atom}(\mu)])$.

. Seja $\mu=\left(s, F, \emptyset, s_{F}, a_{F}, \emptyset\right)$ um tipo "grande" unisortido tal que $F$ é uma classe própria de constantes $\left(\forall f \in F\left(I_{f}=0\right)\right)$. $E S T(\mu, S E T)$ admite limites indutivos (pequenos) $\omega$-filtrados mas não é acessível. ([166])

Pois $\cup_{\kappa<\infty}(E S T(\mu, S E T))_{\kappa}=\emptyset_{\subsetneq}$ obj $(\operatorname{EST}(\mu, S E T))$.

- Seja $X \subseteq V$ classe própria. $\mathcal{D}(X)$ admite limites projetivos/indutivos pequenos, não vazios e conexos mas não é acessível. ([166])

Pois $\forall \omega \leq \kappa<\propto\left(\mathcal{D}\left(X^{\prime}\right)\right)_{\kappa}\left(=\mathcal{D}\left(X^{\prime}\right)\right)$ não é essencialmente pequena.

Em (12) e (13) temos alguns "limites da teoria" apresentada : 
(12) A relação $\unlhd$ na classe dos cardinais regulares infinitos é a "mais geral possível" :

$\forall \kappa . \kappa^{\prime}<\propto$ cardinais regulares infinitos $\left(\forall A \in \operatorname{obj}(C A T)\left(A \kappa\right.\right.$-acessivel $\Rightarrow A \kappa^{\prime}$-acessível )$\left.\Rightarrow \kappa \unlhd \kappa^{\prime}\right)([166])$

. Seja $S E T_{m \in \operatorname{m}}^{\geq \kappa^{\prime}}$ a subcategoria de $S E T$ dos conjuntos de cardinalidade $\geq \kappa^{\prime}$ e mergulhos (funções injetoras). SET $\underset{\bar{m} \epsilon r g}{\geq \kappa^{\prime}}$ é $\kappa$-acessível $\Longleftrightarrow \kappa^{\prime}<\kappa$.

- Seja $O R D_{m \epsilon r g}^{\kappa-d i r}$ a subcategoria de $O R D$ dos conjuntos ordenados $\kappa$-dirigidos e mergulhos (funções injetores que preservam e refletem as relações de ordem). $O R D_{m \in r g}^{\kappa-d i r}$ é $\kappa$-acessível e para os cardinais regulares $\kappa^{\prime} \geq \kappa$ tais que $O R D_{m \in r g}^{\kappa-d i r}$ é $\kappa^{\prime}$-acessível tem-se que: $\forall x$ ( $\# x<\kappa^{\prime} \Rightarrow \exists \mathcal{A} \in\left[[x]^{<\kappa}\right]^{<k^{\prime}}\left((\mathcal{A}, \subseteq) \hookrightarrow\left([x]^{<\kappa}\right)\right)$ é "funtor" final ) ) .

(13) O cardinal $\kappa(\mathcal{S})$ é o "melhor possivel" :

$\exists \mathcal{S} \in \operatorname{obj}(g n-E S Q)\left(\operatorname{MOD}(\mathcal{S}, S E T) \in(\operatorname{obj}(\kappa(\mathcal{S})-A C E))-\bigcup_{\kappa<\kappa(\mathcal{S})}(\operatorname{obj}(\kappa-A C E))\right.$. ([166])

- $S E T_{m \in r g}^{\geq \omega}$ é esboçável por um esboço $\mathcal{S}^{\prime}$ tal que $\pi\left(\mathcal{S}^{\prime}\right)=\tau\left(\mathcal{S}^{\prime}\right)=\omega$ logo: para todo $\omega \leq \tau<\infty$, a categoria produto $A=\left(S E T_{m \in r g}^{\geq \omega}\right)^{\tau}$ é esboçável pelo esboço $\mathcal{S}=\mathcal{S}(\tau) \otimes \mathcal{S}^{\prime}$ $(\mathcal{S}(\tau)$ é o esboço trivial sobre o grafo discreto com $\tau$ objetos) portanto: $\pi(\mathcal{S})=\omega, \tau(\mathcal{S})=\tau$ ,$\kappa(\mathcal{S})=\tau^{+} . A \in(\operatorname{obj}(\kappa(\mathcal{S})-A C E))-\underset{\kappa<\kappa(\mathcal{S})}{\cup}(\operatorname{obj}(\kappa-A C E))$ pois $\underset{\kappa<\kappa(\mathcal{S})}{\cup} A_{\kappa}=\emptyset$.

(14) $[166],[117] ;[075]$

Neste ítem utilizaremos $N B G I N+H$; a extensão (própria) de $N B G U N$ que assume a existência de um cardinal hiperinacessível, i.e. de um ponto fixo regular da função normal que enumera a classe dos cardinais inacessíveis.

Se $\theta_{0}=\# U_{0}$ é um hiperinacessível que não é maior que nenhum cardinal mensurável então existe uma categoria acessível que possui objeto que não admite $<\theta_{0}$ quocientes (não " $\theta_{0}$-co-well-powered").

Seja $A$ a categoria dos modelos transitivos de $Z F C$ que pertencem a $U_{0}$ e mergulhos $F_{\aleph_{1} \aleph_{1}}(\in)$-elementares. Dentre esses modelos destacamos os universos. Temos que:

- Sejam $\mathcal{U}, \mathcal{M}$ objetos de $A \operatorname{com} \mathcal{U}=(U, \in)$ e $U$ universo, então se $\mathcal{H} \in A(\mathcal{U}, \mathcal{M})$ é tal que $\forall \alpha$ ordinal ( $\alpha \in U \Rightarrow H(\alpha)=\alpha)$ então $\forall x(x \in U \Rightarrow H(x)=x)$.

Por indução no $\in$-posto dos elementos de $U$ utilizando que " $\in$-posto" é termo $\Delta_{1}^{Z F C} \mathrm{e}$ que mergulhos $F_{\aleph_{0} \aleph_{0}}(\in)$-elementares preservam termos $\Delta_{1}^{Z F C}$.

. Sejam $\mathcal{U}, \mathcal{M}$ objetos de $A \operatorname{com} \mathcal{U}=(U, \in)$ e $U$ universo, se $\mathcal{H} \in A(\mathcal{U}, \mathcal{M})$ é tal que $\exists x \in U(H(x) \neq x)$ então $\theta=\min \{\alpha: \alpha \in U ; \alpha$ é ordinal $H(\alpha) \neq \alpha\}$ é cardinal mensurável.

Por [117].

- $A$ é acessível.

Seja $B$ a categoria dos modelos bem fundados de $Z F C$ que pertencem a $U_{0}$ e mergulhos $F_{\aleph_{1} \aleph_{1}}(\epsilon)$-elementares. $B$ é acessível pois $Z F C$ é axiomatizável em $L_{\aleph_{0} \aleph_{0}}(\epsilon)$ e " relação $(\epsilon)$ 
bem fundada" é axiomatizável em $L_{\aleph_{1} \aleph_{1}}(\in)$. Portanto $A$ é acessivel pois $A \stackrel{\simeq}{\leftrightarrows} B$ (teorema do isomorfismo de Mostowski).

Consideremos $J: A \hookrightarrow A^{-}$. $\{\emptyset\}$-ind completamento forte de $A$.

. $A^{-}$é acessível.

Pois se $A$ é $\kappa$-acessível então $I:\left(A_{\kappa}\right)^{-} \hookrightarrow A^{-}$é $\mathcal{I}_{\kappa}$-ind completamento forte de categoria essencialmente em $U_{0}$.

. $A^{-}$não é $\theta_{0}$-co-well-powered.

Pois pelo que vimos acima, e pelas hipóteses sobre $\theta_{0}$ temos que $-\infty$ admite $\theta_{0}$ quocientes.

Diferentemente de (14) ; em [166] mostra-se que :

. Toda categoria acessível admitindo "pushouts" (limites indutivos indexados por $\leftarrow \rightarrow$ ) é $\theta_{0}$-co-well-powered.

. Se $\theta_{0}$ é um cardinal hipercompacto então toda categoria acessível é $\theta_{0}$-co-well-powered.

Mais conexões entre categorias acessíveis e hipóteses de existência de grandes cardinais são estabelecidadas em [010] : [004] : [166] , [184] : [006] : [113] ; [189] . 


\section{Bibliografia}

Segue abaixo a relação bibliográfica disponível em nossa biblioteca, em sua maioria.

[001]

S.ABRAMSKY

DOMAIN THEORY IN LOGICAL FORM

ANNALS OF PURE AND APPLIED LOGIC 51 (1991), 1-77

[002]

J.ADÁMEK - H.HERRLICH - J.ROSICKÝ

ESSENTIALLY EQUATIONAL CATEGORIES

CAHIERS DE TOPOLOGIE ET GÉOMÉTRIE DIFFÉRENTIELLE CATÉGORIQUES VOL.XXIX (1988), 175-192

[003]

J.ADÁMEK - J.REITERMAN

EXACTNESS OF THE SET-VALUED COLIM

COMMENTATIONES MATHEMATICAE UNIVERSITATIS CAROLINAE 17 (1976), 97-103

[004]

J.ADÁMEK - J.ROSICKÝ

REFLECTIONS IN LOCALLY PRESENTABLE CATEGORIES

ARCHIVUM MATHEMATICUM VOL.25 (1989), 89-94

[005]

J.ADÁMEK - J.ROSICKÝ

WHAT ARE LOCALLY GENERATED CATEGORIES ?

LECTURE NOTES IN MATHEMATICS 1488, SPRINGER VERLAG (1991), 14-19 [006]

J.ADÁMEK - J.ROSICKÝ

ON INJECTIVITY IN LOCALLY PRESENTABLE CATEGORIES

TRANSACTIONS OF THE AMERICAN MATHEMATICAL SOCIETY VOL.336, (1993): 785-804 
[007]

J.ADÁMEK - J.ROSICKÝ

WEAKLY LOCALLY PRESENTABLE CATEGORIES

CAHIERS DE TOPOLOGIE ET GÉOMÉTRIE DIFFÉRENTIELLE CATÉGORIQUES

VOL.XXXV (1994), 179-186

[008]

J.ADÁMEK - J.ROSICKÝ

ON GEOMETRIC AND FINITARY SKETCHES

APPLIED CATEGORICAL STRUCTURES 4 (1996), 227-240

[009]

J.ADÁMEK - J.ROSICKÝ

FINITE MODELS OF SKETCHES

JOURNAL OF PURE AND APPLIED ALGEBRA 116 (1997); 3-23

[010]

J.ADÁMEK - J.ROSICKÝ - V.TRNKOVÁ

ARE ALL LIMITED CLOSED SUBCATEGORIES OF LOCALLY PRESENTABLE

CATEGORIES REFLECTIVE ?

LECTURE NOTES IN MATHEMATICS 1348, SPRINGER VERLAG (1988), 1-18

[011]

P.AGERON

SÉMANTIQUE CATÉGORIQUE DES TYPES: COMPRENDE LE SYSTÈME $F$

DIAGRAMMES VOL.19 - UNIVERSITÉ PARIS 7 (1988)

[012]

P.AGERON

LES CATÉGORIES LOCALEMENT (MULTI)PRESÉNTABLES

COMME DOMAINES DE SCOTT

DIAGRAMMES VOL.21 - UNIVERSITÉ PARIS 7 (1989)

[013]

P.AGERON

STRUCTURE DES LOGIQUES ET LOGIQUE DES STRUCTURES

THÈSE DE DOCTORAT DE MATHÉMATIQUES - UNIVERSITÉ PARIS 7 (1991)

[014]

P.AGERON

THE LOGIC OF STRUCTURES

JOURNAL OF PURE AND APPLIED ALGEBRA 79 (1992), 15-34

[015]

P.AGERON 
QUELQUES ASPECTS DE LA DUALITÉ ENTRE LOGIQUE ET TOPOLOGIE CAHIERS DE TOPOLOGIE ET GÉOMÉTRIE DIFFÉRENTIELLE CATÉGORIQUES VOL.XXXIII (1992): 195-198

[016]

P.AGERON

LA TOUR HOLOMORPHE D'UNE ESQUISSE

CAHIERS DE TOPOLOGIE ET GÉOMÉTRIE DIFFÉRENTIELLE CATÉGORIQUES VOL.XXXVII (1996), 295-314

[017]

M.H.ALBERT - G.M.KELLY

THE CLOSURE OF A CLASS OF COLIMITS

JOURNAL OF PURE AND APPLIED ALGEBRA 51 (1988), 1-17

[018]

H.ANDRÉKA - Á.KURUCZ - I.NÉMETI

CONNECTIONS BETWEEN AXIOMS OF SET THEORY AND BASIC

THEOREMS OF UNIVERSAL ALGEBRA

THE JOURNAL OF SYMBOLIC LOGIC VOL.59 (1994), 912-923

[019]

M.BARR

COEQUALIZERS AND FREE TRIPLES

MATHEMATISCHE ZEITSCHRIFT 116 (1970), 307-322

[020]

M.BARR

EXACT CATEGORIES

LECTURE NOTES IN MATHEMATICS 236, SPRINGER VERLAG (1971), 1-120

[021]

M.BARR

MODELS OF SKETCHES

CAHIERS DE TOPOLOGIE ET GÉOMÉTRIE DIFFÉRENTIELLE CATÉGORIQUES VOL.XXVII (1986), 93-107

[022]

M.BARR

MODELS OF HORN THEORIES

CONTEMPORARY MATHEMATICS 92 .

AMERICAN MATHEMATICAL SOCIETY (1989), 1-7

[023]

M.BARR 
ACCESSIBLE CATEGORIES AND MODELS OF LINEAR LOGIC

JOURNAL OF PURE AND APPLIED ALGEBRA 69 (1990), 219-232

[024]

M.BARR

FUNCTORIAL SEMANTICS AND HSP TYPE THEOREMS

ALGEBRA UNIVERSALIS 31 (1994), 223-251

[025]

M.BARR - M.PEDICCHIO

TOP ${ }^{o p}$ IS A QUASI-VARIETY

CAHIERS DE TOPOLOGIE ET GÉOMÉTRIE DIFFÉRENTIELLE CATÉGORIQUES

VOL.XXXVI (1995), 3-10

[026]

M.BARR - C.WELLS

TOPOSES, TRIPLES AND THEORIES

GRUNDLEHREN DER MATHEMATISCHEN WISSENSCHAFTEN 278

SPRINGER VERLAG (1985)

[027]

M.BARR - C.WELLS

CATEGORY THEORY FOR COMPUTING SCIENCE

PRENTICE HALL (1990)

[028]

A.BASTIANI

SKETCHED STRUCTURES AND COMPLETIONS

CAHIERS DE TOPOLOGIE ET GÉOMÉTRIE DIFFÉRENTIELLE

VOL.XIV (1973), 158-160

[029]

A.BASTIANI - C.EHRESMANN

CATEGORIES OF SKETCHED STRUCTURES

CAHIERS DE TOPOLOGIE ET GÉOMÉTRIE DIFFÉRENTIELLE

VOL.XIII (1972), 104-214

[030]

A.BASTIANI - C.EHRESMANN

MULTIPLE FUNCTORS : I. LIMITS RELATIVE TO DOUBLE CATEGORIES

CAHIERS DE TOPOLOGIE ET GÉOMÉTRIE DIFFÉRENTIELLE

VOL.XV (1974), 215-292

[031]

J.BÉNABOU 
INTRODUCTION TO BICATEGORIES

LECTURE NOTES IN MATHEMATICS 47. SPRINGER VERLAG (1967), 1-77

[032]

J.BÉNABOU

STRUCTURES ALGÉBRIQUES DANS LE CATÉGORIES

CAHIERS DE TOPOLOGIE ET GÉOMÉTRIE DIFFÉRENTIELLE

VOL.X (1969), 1-126

[033]

R.BETTI - M.GRANDIS

COMPLETE THEORIES IN 2-CATEGORIES

CAHIERS DE TOPOLOGIE ET GÉOMÉTRIE DIFFÉRENTIELLE CATÉGORIQUES VOL.XXIX (1988), 9-57

[034]

G.J.BIRD - G.M.KELLY - A.J.POWER- R.H.STREET

FLEXIBLE LIMITS FOR 2-CATEGORIES

JOURNAL OF PURE AND APPLIED ALGEBRA 61 (1989), 1-27

[035]

G.BIRKHOFF

ON THE STRUCTURE OF ABSTRACT ALGEBRAS

PROCEEDINGS OF THE CAMBRIDGE PHILOSOPHICAL SOCIETY

VOL.31 (1935), 433-454

[036]

A.A.BLANCHARD

STRUCTURE SPECIES AND CONSTRUCTIVE FUNCTORS

CANADIAN JOURNAL OF MATHEMATICS VOL.XXVI (1974): 1217-1227

[037]

A.BLASS

THE INTERACTION BETWEEN CATEGORY THEORY AND SET THEORY CONTEMPORARY MATHEMATICS 30 ,

AMERICAN MATHEMATICAL SOCIETY (1984), 5-29

[038]

F.BORCEUX - G.V.BOSSCHE

ALGEBRA IN LOCALIC TOPOS WITH APPLICATIONS TO RING THEORY LECTURE NOTES IN MATHEMATICS 1038, SPRINGER VERLAG (1983) [039]

F.BORCEUX - B.J.DAY

ON CHARACTERISATION OF FINITARY ALGEBRAIC CATEGORIES 
BULLETIN OF THE AUSTRALIAN MATHEMATICAL SOCIETY VOL.18

(1978), $125-135$

[040]

R.BÖRGER - W.THOLEN

ABSCHWÄCHUNGEN DES ADJUNKTIONSBEGRIFFS

MANUSCRIPTA MATHEMATICA 19 (1976), 19-45

[041]

R.BÖRGER - W.THOLEN

CANTORS DIAGONALPRINZIP FÜR KATEGORIEN

MATHEMATISCHE ZEITSCHRIFT 160 (1978), 135-138

[042]

R.BÖRGER - W.THOLEN

STRONG, REGULAR AND DENSE GENERATORS

CAHIERS DE TOPOLOGIE ET GÉOMÉTRIE DIFFÉRENTIELLE CATÉGORIQUES

VOL.XXXII (1991), 257-276

[043]

N.BOURBAKI

ELEMENTS OF MATHEMATICS: THEORY OF SETS

ADDISON WESLEY (HERMANN) (1968)

[044]

M.C.BUNGE

RELATIVE FUNCTOR CATEGORIES AND CATEGORIES OF ALGEBRAS

JOURNAL OF ALGEBRA 11 (1969), 64-101

[045]

A.BURRONI

ESQUISSES DE CATÉGORIES À LIMITES ET DES QUASI-TOPOLOGIES

ESQUISSES MATHÉMATIQUES 5 (1970)

[046]

E.BURRONI - A.BURRONI

STRUCTURES ALGÉBRIQUES: THÈME ET VARIATIONS

CAHIERS DE TOPOLOGIE ET GÉOMÉTRIE DIFFÉRENTIELLE CATÉGORIQUES VOL. XXXIII (1992), 207-216

[047]

J.CARTMELL

GENERALISED ALGEBRAIC THEORIES AND CONTEXTUAL CATEGORIES

ANNALS OF PURE AND APPLIED LOGIC 32 (1986), 209-243

[048] 
C.C.CHANG - H.J.KEISLER

MODEL THEORY

STUDIES IN LOGIC AND FOUNDATIONS OF MATHEMATICS VOL.73,

NORTH HOLLAND (1990)

[049]

F.CONDUCHE

SUR LES STRUCTURES DÉFINIES PAR LIMITES PROJECTIVES

ESQUISSES MATHÉMATIQUES 11 (1971)

[050]

L.COPPEY

ESQUISSES ET TYPES

DIAGRAMMES VOL.27 - UNIVERSITÉ PARIS 7 (1992)

[051]

L.COPPEY - C.LAIR

LEÇONS DE THÉORIE DES ESQUISSES (II)

DIAGRAMMES VOL.19 - UNIVERSITÉ PARIS 7 (1988)

[052]

T.COQUAND

CATEGORIES OF EMBEDDINGS

THEORETICAL COMPUTER SCIENCE 68 (1989), 221-237

[053]

N.C.A.COSTA - A.J.CAROLI

REMARQUES SUR LES UNIVERS D'EHRESMANN-DEDECKER

COMPTES RENDUS DE L'ACADÉMIE DES SCIENCES (PARIS)

T.265, SERIE A (1967), 761-763

[054]

M.COSTE

LOCALISATION, SPECTRA AND SHEAF REPRESENTATION

LECTURE NOTES IN MATHEMATICS 753, SPRINGER VERLAG (1979), 212-238

[055]

F.CURY

COMPLÉTION ET COMPLÉTUDE, SELON H.ANDRÉKA ET I.NÉMETI

DIAGRAMMES VOL.28 - UNIVERSITÉ PARIS 7 (1992)

[056]

B.J.DAY

DENSITY PRESENTATIONS OF FUNCTORS

BULLETIN OF THE AUSTRALIAN MATHEMATICAL SOCIETY VOL.16 
(1977), $427-448$

[057]

B.DAY - R.STREET

LOCALISATION OF LOCALLY PRESENTABLE CATEGORIES

JOURNAL OF PURE AND APPLIED ALGEBRA 58 (1989), 227-233

[058]

M.A.DICKMANN

LARGE INFINITARY LANGUAGES

STUDIES IN LOGIC AND THE FOUNDATIONS OF MATHEMATICS VOL.83,

NORTH HOLLAND (1975)

[059]

K.H.DIENER

AN APPLICATION OF INFINITARY UNIVERSAL ALGEBRA TO SET THEORY ALGEBRA UNIVERSALIS 32 (1994), 297-306

[060]

Y.DIERS

J-ADJONCTION. LIMITE J-ABSOLUTE ET J-THÉORIE ALGÉBRIQUE

COMPTES RENDUS DE L'ACADÉMIE DES SCIENCES (PARIS)

T.278, SERIE A (1974), 1009-1012

[061]

Y.DIERS

FONCTEUR PLEINEMENT FIDĖLE DENSE CLASSANT LES ALGĖBRES

CAHIERS DE TOPOLOGIE ET GÉOMÉTRIE DIFFÉRENTIELLE

VOL.XVII (1976), 171-186

[062]

Y.DIERS

VARIÉTES D'UNE CATÉGORIE

ANNALES DE LA SOCIÉTÉ SCIENTIFIQUE DE

BRUXELLES T.90 (1976), 159-172

[063]

Y.DIERS

CATÉGORIES LOCALISABLES

THÈSE DE DOCTORAT - UNIVERSITÉ PARIS 7 (1977)

[064]

Y.DIERS

SPECTRES ET LOCALISATIONS RELATIFS À UN FONTEUR

COMPTES RENDUS DE L'ACADÉMIE DES SCIENCES (PARIS) 
T.287. SERIE A (1978), 985-988

[065]

Y.DIERS

FAMILLES UNIVERSELLES DE MORPHISMES

ANNALES DE LA SOCIÉTÉ SCIENTIFIQUE DE

BRUXELLES T.93 (1979), 175-195

[066]

Y.DIERS

CATÉGORIES MULTIALGÉBRIQUES

ARCHIV DER MATHEMATIK VOL.34 (1980), 193-209

[067]

Y.DIERS

CATÉGORIES LOCALEMENT MULTIPRÉSENTABLES

ARCHIV DER MATHEMATIK VOL.34 (1980), 344-356

[068]

Y.DIERS

MULTIMONADS AND MULTIMONADIC CATEGORIES

JOURNAL OF PURE AND APPLIED ALGEBRA 17 (1980), 153-170

[069]

Y.DIERS

SOME SPECTRA RELATIVE TO FUNCTORS

JOURNAL OF PURE AND APPLIED ALGEBRA 22 (1981), 57-74

[070]

Y.DIERS

UN CRITÉRE DE REPRÉSENTABILITÉ PAR SECTIONS

CONTINUES DE FAISCEAUX

LECTURE NOTES IN MATHEMATICS 962, SPRINGER VERLAG (1982), 47-61 [071]

Y.DIERS

CATEGORIES OF BOOLEAN SHEAVES OF SIMPLE ALGEBRAS

LECTURE NOTES IN MATHEMATICS 1187. SPRINGER VERLAG (1986)

[072]

Y.DIERS

CATÉGORIES ALGÉBRIQUES PREGALOISIENNES

CAHIERS DE TOPOLOGIE ET GÉOMÉTRIE DIFFÉRENTIELLE CATÉGORIQUES VOL.XXXII (1991), 279-296

[073] 
Y.DIERS

CATÉGORIES MULTIALGÉBRIQUES GALOISIENNES

CAHIERS DE TOPOLOGIE ET GÉOMÉTRIE DIFFÉRENTIELLE CATÉGORIQUES VOL.XXXIII (1992), 55-69

[074]

Y.DIERS

CATEGORIES OF ALGEBRAIC SETS

APPLIED CATEGORICAL STRUCTURES 4 (1996), 329-341

[075]

F.R.DRAKE

SET THEORY - AN INTRODUCTION TO LARGE CARDINALS

STUDIES IN LOGIC AND THE FOUNDATIONS OF MATHEMATICS VOL.76,

NORTH HOLLAND (1974)

[076]

M.DROSTE

EVENT STRUCTURES AND DOMAINS

THEORETICAL COMPUTER SCIENCE 68 (1989), 37-47

[077]

C.EHRESMANN

SUR L'EXISTENCE DE STRUCTURES LIBRES ET DE FONCTEURS ADJOINTS

CAHIERS DE TOPOLOGIE ET GÉOMÉTRIE DIFFÉRENTIELLE

VOL.IX (1967), 33-180

[078]

C.EHRESMANN

ESQUISSES ET TYPES DE STRUCTURES ALGÉBRIQUES

BULETINUL INSTITUTULUI POLITEHNIC DIN IASI T.XIV (1968), 1-14

[079]

C.EHRESMANN

CONSTRUCTION DE STRUCTURES LIBRES

LECTURE NOTES IN MATHEMATICS 92, SPRINGER VERLAG (1969), 74-104

[080]

P.C.EKLOF

ON CONSTRUCTING INDECOMPOSABLE GROUPS IN $L$

JOURNAL OF ALGEBRA 49 (1977), 96-103

[081]

S.FAKIR

OBJECTS ALGÉBRIQUEMÉNT CLOS ET INJECTIFS DANS 
LES CATÉGORIES LOCALEMENT PRÉSENTABLES

BULLETIN DE LA SOCIÉTÉ MATHÉMATIQUE DE FRANCE

MÉMOIRE N42 (1975)

[082]

S.FAKIR - L.HADDAD

OBJECTS COHÉRENTS ET ULTRAPRODUITS DANS LES CATÉGORIES

JOURNAL OF ALGEBRA 21 (1972): 410-421

[083]

E.R.FISHER

VOPĚNKA:S PRINCIPLE, CATEGORY THEORY AND UNIVERSAL ALGEBRA

NOTICES OF AMERICAN MATHEMATICAL SOCIETY 24 (1977), A-44

[084]

F.FOLTZ

SUR LA COMUTATION DES LIMITES

DIAGRAMMES 5 - UNIVERSITÉ PARIS 7 (1981)

[085]

F.FOLTZ - C.LAIR

FERMETURE STANDARD DES CATÉGORIES ALGÉBRIQUES

CAHIERS DE TOPOLOGIE ET GÉOMÉTRIE DIFFÉRENTIELLE

VOL.XIII (1972), 275-307

[086]

F.FOLTZ - C.LAIR - G.M.KELLY

ALGEBRAIC CATEGORIES WITH FEW MONOIDAL BICLOSED STRUCTURES OR NONE

JOURNAL OF PURE AND APPLIED ALGEBRA 17 (1980), 171-177

[087]

T.F.FOX

PURITY IN LOCALLY-PRESENTABLE MONOIDAL CATEGORIES

JOURNAL OF PURE AND APPLIED ALGEBRA 8 (1976), 261-265

[088]

P.J.FREYD

PROPERTIES INVARIANT WITHIN EQUIVALENCE TYPES OF CATEGORIES

ALGEBRA, TOPOLOGY AND CATEGORY THEORY, ACADEMIC PRESS (1976), $55-61$

[089]

P.J.FREYD - G.M.KELLY

CATEGORIES OF CONTINUOUS FUNCTORS, I 
JOURNAL OF PURE AND APPLIED ALGEBRA 2 (1972): 169-191

[090]

P.J.FREYD - G.M.KELLY

CATEGORIES OF CONTINUOUS FUNCTORS, I - ERRATUM

JOURNAL OF PURE AND APPLIED ALGEBRA 4 (1974): 121

[091]

P.J.FREYD - A.SCEDROV

CATEGORIES, ALLEGORIES

NORTH HOLLAND MATHEMATICAL LIBRARY VOL.39, NORTH HOLLAND (1990) [092]

P.GABRIEL - F.ULMER

LOKAL PRÄSENTIERBARE KATEGORIEN

LECTURE NOTES IN MATHEMATICS 221, SPRINGER VERLAG (1971)

[093]

M.GERNER - R.GUITART

THE LOCALLY FREE RELATIVELY FILTERED DIAGRAM AS

AN INDUCTIVE COMPLETION OF A SYSTEM OF CHOICE

APPLIED CATEGORICAL STRUCTURES 5 (1997), 59-73

[094]

G.GIERZ - K.H.HOFMANN - K.KEIMEL - J.D.LAWSON - M.MISLOVE - D.S.SCOTT

A COMPENDIUM OF CONTINUOUS LATTICES

SPRINGER VERLAG (1980)

[095]

J.W.GRAY

CATEGORICAL ASPECTS OF DATA TYPE CONSTRUCTORS

THEORETICAL COMPUTER SCIENCE 50 (1987): 103-135

[096]

J.W.GRAY

THE CATEGORY OF SKETCHES AS A MODEL FOR ALGEBRAIC SEMANTICS CONTEMPORARY MATHEMATICS 92,

AMERICAN MATHEMATICAL SOCIETY (1989): 109-135

[097]

J.W.GRAY

EXECUTABLE SPECIFICATIONS FOR DATA-TYPE CONSTRUCTORS

DIAGRAMMES VOL.24 - UNIVERSITÉ PARIS 7 (1990)

[098]

A.GROTHENDIECK - J.L.VERDIER 
PREFAISCEAU (APPENDICE: UNIVERS (N.BOURBAKI))

LECTURE NOTES IN MATHEMATICS 269, SPRINGER VERLAG (1972): 1-217

[099]

R.GUITART

QU'EST-CE QUE LA LOGIQUE DANS UNE CATÉGORIE ?

CAHIERS DE TOPOLOGIE ET GÉOMÉTRIE DIFFÉRENTIELLE

VOL.XXIII (1982), 115-148

[100]

R.GUITART

ON THE GEOMETRY OF COMPUTATIONS

CAHIERS DE TOPOLOGIE ET GÉOMÉTRIE DIFFÉRENTIELLE

CATÉGORIQUES

VOL.XXVII (1986), 107-136

[101]

R.GUITART

ON THE GEOMETRY OF COMPUTATIONS, II

CAHIERS DE TOPOLOGIE ET GÉOMÉTRIE DIFFÉRENTIELLE CATÉGORIQUES VOL.XXIX (1988), 297-326

[102]

R.GUITART

L'UNITÉ DE LA THÉORIE DES MODĖLES ET DE L'ALGÈBRE HOMOLOGIQUE CAHIERS DE TOPOLOGIE ET GÉOMÉTRIE DIFFÉRENTIELLE CATÉGORIQUES VOL.XXX (1989), 281-293

[103]

R.GUITART - C.LAIR

CALCUL SYNTAXIQUE DES MODĖLES ET CALCUL DES FORMULES INTERNES DIAGRAMMES VOL.4 - UNIVERSITÉ PARIS 7 (1980)

[104]

R.GUITART - C.LAIR

EXISTENCE DE DIAGRAMMES LOCALEMENT LIBRES

DIAGRAMMES VOL.6 - UNIVERSITÉ PARIS 7 (1981)

[105]

R.GUITART - C.LAIR

EXISTENCE DE DIAGRAMMES LOCALEMENT LIBRES, II

DIAGRAMMES VOL.7 - UNIVERSITÉ PARIS 7 (1982)

[106]

R.GUITART - C.LAIR 
LIMITES ET COLIMITES POUR REPRÉSENTER LES FORMULES

DIAGRAMMES VOL.7 - UNIVERSITÉ PARIS 7 (1982)

[107]

R.GUITART - J.RIGUET

ENVELOPPE KAROUBIENNE DE CATÉGORIES DE KLEISLI

CAHIERS DE TOPOLOGIE ET GÉOMÉTRIE DIFFÉRENTIELLE CATÉGORIQUES VOL.XXXIII (1992), 261-266

[108]

C.A.GUNTER - A.JUNG

COHERENCE AND CONSISTENCY IN DOMAINS

JOURNAL OF PURE AND APPLIED ALGEBRA 63 (1990), 49-66

[109]

M.HÉBERT

SYNTACTIC CHARACTERIZATION OF CLOSURE UNDER CONNECTED LIMITS ARCHIVE FOR MATHEMATICAL LOGIC 31 (1991), 133-143

[110]

H.HERRLICH

ON THE FAILURE OF BIRKHOFF:S THEOREM FOR

LOCALLY SMALL BASED EQUATIONAL CATEGORIES OF ALGEBRAS

CAHIERS DE TOPOLOGIE ET GÉOMÉTRIE DIFFÉRENTIELLE CATÉGORIQUES VOL.XXXIV (1993), 185-192

[111]

P.J.HILTON

ON FILTERED SYSTEMS OF GROUPS, COLIMITS AND KAN EXTENSIONS JOURNAL OF PURE AND APPLIED ALGEBRA VOL.1 (1971), 199-217

[112]

W.HODGES

THE MEANING OF SPECIFICATIONS I: DOMAINS AND INITIAL MODELS

THEORETICAL COMPUTER SCIENCE 152 (1995); 67-89

[113]

H.HU - M.MAKKAI

ACCESSIBLE EMBEDDINGS AND THE SOLUTION-SET CONDITION

CAHIERS DE TOPOLOGIE ET GÉOMÉTRIE DIFFÉRENTIELLE CATÉGORIQUES VOL.XXXV (1994), 99-108

[114]

H.HU - W.THOLEN

QUASI-COPRODUCTS AND ACCESSIBLE CATEGORIES WITH 
WIDE PULLBACKS

APPLIED CATEGORICAL STRUCTURES 4 (1996). 387-402

[115]

\section{A.HUQ}

A NOTE ON GENERATORS IN CATEGORIES

CAHIERS DE TOPOLOGIE ET GÉOMÉTRIE DIFFÉRENTIELLE

VOL.XIII (1972), 393-395

[116]

J.R.ISBELL

GENERAL FUNCTORIAL SEMANTICS, I

AMERICAN JOURNAL OF MATHEMATICS 94 (1972), 535-596

[117]

T.JECH

SET THEORY

ACADEMIC PRESS (1978)

[118]

M.JOHNSON - R.F.C.WALTERS

ALGEBRA OBJECTS AND ALGEBRA FAMILIES FOR FINITE LIMIT THEORIES JOURNAL OF PURE AND APPLIED ALGEBRA 83 (1992), 283-293

[119]

P.T.JOHNSTONE

RINGS, FIELDS, AND SPECTRA

JOURNAL OF ALGEBRA 49 (1977), 238-260

[120]

P.T.JOHNSTONE

A SYNTATIC APPROACH TO DIERS' LOCALIZABLE CATEGORIES

LECTURE NOTES IN MATHEMATICS 753, SPRINGER VERLAG (1980), 466-478

[121]

P.T.JOHNSTONE

STONE SPACES

CAMBRIDGE UNIVERSITY PRESS (1982)

[122]

A.JUNG

CARTESIAN CLOSED CATEGORIES OF ALGEBRAIC CPOs

THEORETICAL COMPUTER SCIENCE 70 (1990), 233-250

[123]

J.J.KAPUT 
LOCALLY ADJUNCTABLE FUNCTORS

ILLINOIS JOURNAL OF MATHEMATICS 16 (1972). \$6-94

[124]

O.KEAN

ABSTRACT HORN THEORIES

LECTURE NOTES IN MATHEMATICS 445. SPRINGER VERLAG (1975), 15-50 [125]

G.M.KELLY

QUELQUES OBSERVATIONS SUR LES DEMONSTRATIONS

PAR RECURRENCE TRANSFINIE EN ALGĖBRE CATÉGORIQUE

CAHIERS DE TOPOLOGIE ET GÉOMÉTRIE DIFFÉRENTIELLE

VOL.XVI (1975), 259-263

[126]

G.M.KELLY

A UNIFIED TREATMENT OF TRANSFINITE CONSTRUCTIONS FOR FREE ALGEBRAS. FREE MONOIDS. COLIMITS. ASSOCIATED SHEAVES, AND SO ON

BULLETIN OF THE AUSTRALIAN MATHEMATICAL SOCIETY VOL.22 (1980), 183

[127]

G.M.KELLY

ON THE ESSENTIALLY-ALGEBRAIC THEORY GENERATED BY A SKETCH

BULLETIN OF THE AUSTRALIAN MATHEMATICAL SOCIETY VOL.26 (1982), 45-56

[128]

G.M.KELLY

STRUCTURES DEFINED BY FINITE LIMITS IN THE ENRICHED CONTEXT. I

CAHIERS DE TOPOLOGIE ET GÉOMÉTRIE DIFFÉRENTIELLE

VOL.XXIII (1982), 3-42

[129]

G.M.KELLY

A NOTE ON THE GENERALIZED REFLEXION OF GUITART AND LAIR

CAHIERS DE TOPOLOGIE ET GÉOMÉTRIE DIFFÉRENTIELLE

VOL.XXIV (1983), 155-159

[130]

G.M.KELLY - R.PARÉ

A NOTE ON THE ALBERT-KELLY PAPER

"THE CLOSURE OF A CLASS OF COLIMITS" 
JOURNAL OF PURE AND APPLIED ALGEBRA 51 (1988), 19-25

[131]

G.M.KELLY - A.J.POWER

ADJUNCTIONS WHOSE COUNITS ARE COEQUALIZERS,

AND PRESENTATIONS OF FINITARY ENRICHED MONADS

JOURNAL OF PURE AND APPLIED ALGEBRA 89 (1993), 163-179

[132]

J.F.KENNISON

ON LIMIT-PRESERVING FUNCTORS

ILLINOIS JOURNAL OF MATHEMATICS 12 (1968), 616-619

[133]

Y.KINOSHITA - J.POWER

LAX NATURALITY THROUGH ENRICHMENT

JOURNAL OF PURE AND APPLIED ALGEBRA 112 (1996), 53-72

[134]

A.KOCK

MONADS FOR WHICH STRUCTURES ARE ADJOINT TO UNITS

JOURNAL OF PURE AND APPLIED ALGEBRA 104 (1995), 41-59

[135]

A.KOCK - G.E.REYES

DOCTRINES IN CATEGORICAL LOGIC

HANDBOOK OF MATHEMATICAL LOGIC, NORTH HOLLAND (1977), 283-313

[136]

V.KOUBEK - J.REITERMAN

CATEGORICAL CONSTRUCTIONS OF FREE ALGEBRAS,

COLIMITS, AND COMPLETIONS OF PARTIAL ALGEBRAS

JOURNAL OF PURE AND APPLIED ALGEBRA 14 (1979). 195-231

[137]

L.KUČERA - A.PULTR

NON-ALGEBRAIC CONCRETE CATEGORIES

JOURNAL OF PURE AND APPLIED ALGEBRA 3 (1973), 95-102

[138]

C.LAIR

CONSTRUCTIONS D:ESQUISSES. TRANSFORMATIONS NATURELLES

GÉNÉRALISÉES

ESQUISSES MATHÉMATIQUES 2 (1970)

[139] 


\section{C.LAIR}

FONCTEURS D:OMISSION DE STRUCTURES ALGÉBRIQUES

CAHIERS DE TOPOLOGIE ET GÉOMÉTRIE DIFFÉRENTIELLE VOL.XII (1971), 147-186

[140]

C.LAIR

DUALITÉS POUR LES STRUCTURES ALGÉBRIQUES ESQUISSÉES CAHIERS DE TOPOLOGIE ET GÉOMÉTRIE DIFFÉRENTIELLE VOL.XIV (1973): 192-193

[141]

C.LAIR

DUALITÉS POUR LES STRUCTURES ALGÉBRIQUES ESQUISSÉES

CAHIERS DE TOPOLOGIE ET GÉOMÉTRIE DIFFÉRENTIELLE VOL.XV (1974): 353-376

[142]

C.LAIR

ESQUISSABILITÉ ET TRIPLABILITÉ

CAHIERS DE TOPOLOGIE ET GÉOMÉTRIE DIFFÉRENTIELLE VOL.XVI (1975), 274-279

[143]

C.LAIR

FERMETURE STANDARD DES CATÉGORIES ALGÉBRIQUES II CAHIERS DE TOPOLOGIE ET GÉOMÉTRIE DIFFÉRENTIELLE VOL.XVIII (1977), 3-60

[144]

C.LAIR

CONDITION SYNTAXIQUE DE TRIPLABILITÉ

D’UN FONCTEUR ALGÉBRIQUE ESQUISSÉ

DIAGRAMMES VOL.1 - UNIVERSITÉ PARIS 7 (1979)

[145]

C.LAIR

CATÉGORIES MODELABLES ET CATÉGORIES ESQUISSABLES

DIAGRAMMES VOL.6 - UNIVERSITÉ PARIS 7 (1981)

[146]

C.LAIR

A PROPOS DE "TOPOSES, TRIPLES AND THEORIES"

DE MESSIEURS M.BARR ET C.WELLS 
DIAGRAMMES VOL.15 - UNIVERSITÉ PARIS 7 (1986)

[147]

C.LAIR

CATÉGORIES QUALIFIABLES ET CATÉGORIES ESQUISSABLES

DIAGRAMMES VOL.17 - UNIVERSITÉ PARIS 7 (1987)

[148]

C.LAIR

DIAGRAMMES STRUCTURÉS DE MODĖLES

DIAGRAMMES VOL.18 - UNIVERSITÉ PARIS 7 (1987)

[149]

C.LAIR

TRAMES ET SÉMANTIQUES CATÉGORIQUES DES SYSTÈMES DE TRAMES

DIAGRAMMES VOL.18 - UNIVERSITÉ PARIS 7 (1987)

[150]

C.LAIR

ELÉMENTS DE THÉORIE DES PATCHWORKS (I)

DIAGRAMMES VOL.29 - UNIVERSITÉ PARIS 7 (1993)

[151]

J.LAMBEK

COMPLETIONS OF CATEGORIES

LECTURE NOTES IN MATHEMATICS 24, SPRINGER VERLAG (1966)

[152]

F.W.LAWVERE

FUNCTORIAL SEMANTICS OF ALGEBRAIC THEORIES

PROCEEDINGS OF NATIONAL ACADEMY OF SCIENCES (USA) 50 (1963), 869-872 [153]

F.W.LAWVERE

SOME ALGEBRAIC PROBLEMS IN THE CONTEXT OF

FUNCTORIAL SEMANTICS OF ALGEBRAIC THEORIES

LECTURE NOTES IN MATHEMATICS 61, SPRINGER VERLAG (1968), 41-61

[154]

F.W.LAWVERE

ORDINAL SUMS AND EQUATIONAL DOCTRINES

LECTURE NOTES IN MATHEMATICS 80. SPRINGER VERLAG (1969), 141-155

[155]

S.K.LELLAHI

CATEGORICAL ABSTRACT DATA TYPE (CADT) 
DIAGRAMMES VOL.21 - UNIVERSITÉ PARIS 7 (1989)

[156]

L.LIBKIN

A REMARK ABOUT ALGEBRAICITY IN COMPLETE PARTIAL ORDERS

JOURNAL OF PURE AND APPLIED ALGEBRA 86 (1993), 75-77

[157]

F.E.J.LINTON

SOME ASPECTS OF EQUATIONAL CATEGORIES

PROCEEDINGS OF THE LA JOLLA CONFERENCE ON CATEGORICAL ALGEBRA:

SPRINGER VERLAG (1966), 84-94

[158]

F.E.J.LINTON

AN OUTLINE OF FUNCTORIAL SEMANTICS

LECTURE NOTES IN MATHEMATICS 80, SPRINGER VERLAG (1968), 7-52 [159]

S.MACLANE

CATEGORIES FOR THE WORKING MATHEMATICIAN

GRADUATE TEXTS IN MATHEMATICS 5, SPRINGER VERLAG (1971)

[160]

M.MAKKAI

ON FULL EMBEDDINGS I

JOURNAL OF PURE AND APPLIED ALGEBRA 16 (1980); 183-195

[161]

M.MAKKAI

STRONG CONCEPTUAL COMPLETENESS FOR FIRST-ORDER LOGIC ANNALS OF PURE AND APPLIED LOGIC 40 (1988): 167-215

[162]

M.MAKKAI

A THEOREM ON BARR-EXACT CATEGORIES, WITH AN INFINITARY GENERALIZATION

ANNALS OF PURE AND APPLIED LOGIC 47 (1990), 225-268

[163]

M.MAKKAI

GENERALIZED SKETCHES AS A FRAMEWORK FOR

COMPLETENESS THEOREMS. PART I

JOURNAL OF PURE AND APPLIED ALGEBRA 115 (1997): 49-79 
[164]

M.MAKKAI

GENERALIZED SKETCHES AS A FRAMEWORK FOR

COMPLETENESS THEOREMS. PART II

JOURNAL OF PURE AND APPLIED ALGEBRA 115 (1997). 179-192

[165]

M.MAKKAI

GENRALIZED SKETCHES AS A FRAMEWORK FOR

COMPLETENESS THEOREMS. PART III

JOURNAL OF PURE AND APPLIED ALGEBRA 115 (1997), 241-274

[166]

M.MAKKAI - R.PARÉ

ACCESSIBLE CATEGORIES: THE FOUNDATIONS OF CATEGORICAL MODEL THEORY

CONTEMPORARY MATHEMATICS 104.

AMERICAN MATHEMATICAL SOCIETY (1989)

[167]

M.MAKKAI - A.M.PITTS

SOME RESULTS ON LOCALLY FINITELY PRESENTABLE CATEGORIES

TRANSACTIONS OF THE AMERICAN MATHEMATICAL SOCIETY

VOL.299 (1987), 473-496

[168]

M.MAKKAI - G.E.REYES

FIRST ORDER CATEGORICAL LOGIC

LECTURE NOTES IN MATHEMATICS 611, SPRINGER VERLAG (1977)

[169]

J.A.MAKOWSKY

VOPĔNKA:S PRINCIPLE AND COMPACT LOGICS

THE JOURNAL OF SYMBOLIC LOGIC VOL.50 (1985), 42-48

[170]

E.G.MANES

ALGEBRAIC THEORIES

GRADUATE TEXTS IN MATHEMATICS 26. SPRINGER VERLAG (1976)

[171]

J.MARTIN - E.HYLAND - A.M.PITTS

THE THEORY OF CONSTRUCTIONS: CATEGORICAL SEMANTICS

AND TOPOS-THEORETIC MODELS 
CONTEMPORARY MATHEMATICS 92.

AMERICAN MATHEMATICAL SOCIETY (1989): 137-199

[172]

C.McLARTY

LEFT EXACT LOGIC

JOURNAL OF PURE AND APPLIED ALGEBRA 41 (1986), 63-66

[173]

G.MICHON

COALGĖBRE ET EXTENSION DE KAN

COMPTES RENDUS DE L'ACADÉMIE DES SCIENCES (PARIS)

T.276, SERIE A (1973), 1091-1093

[174]

F.MOUEN

SUR LA CARACTERISATION SÉMANTIQUE DES CATÉGORIES

DE STRUCTURES

DIAGRAMMES VOL.11 - UNIVERSITÉ PARIS 7 (1984)

[175]

R.PARÉ

CONNECTED COMPONENTS AND COLIMITS

JOURNAL OF PURE AND APPLIED ALGEBRA 3 (1973), 21-42

[176]

R.PARÉ

SOME APPLICATIONS OF CATEGORICAL MODEL THEORY

CONTEMPORARY MATHEMATICS 92.

AMERICAN MATHEMATICAL SOCIETY (1989), 325-339

[177]

R.PARÉ

SIMPLY CONNECTED LIMITS

CANADIAN JOURNAL OF MATHEMATICS VOL.XLII (1990), 731-746

[178]

M.C.PEDICCHIO - W.THOLEN

LOCALIZATIONS OF LOCALLY PRESENTABLE CATEGORIES

AND EXACT TOPOLOGIES

JOURNAL OF PURE AND APPLIED ALGEBRA 64 (1990), 293-301

[179]

F.PIESSENS - E.STEEGMANS

PROVING SEMANTICAL EQUIVALENCE OF DATA SPECIFICATIONS 
JOURNAL OF PURE AND APPLIED ALGEBRA 116 (1997), 291-322

[180]

J.ROSICKÝ

CATEGORIES OF MODELS OF INFINITARY HORN THEORIES

ARCHIVUM MATHEMATICUM VOL.XIV (1978), 219-226

[181]

J.ROSICKÝ

CONCRETE CATEGORIES AND INFINITARY LANGUAGES

JOURNAL OF PURE AND APPLIED ALGEBRA 22 (1981), 309-339

[182]

J.ROSICKÝ

SEMI-INITIAL COMPLETIONS

JOURNAL OF PURE AND APLLIED ALGEBRA 40 (1986), 177-183

[183]

J.ROSICKÝ

MODELS OF HORN THEORIES REVISITED

JOURNAL OF PURE AND APPLIED ALGEBRA 92 (1994), 185-190

[184]

J.ROSICKÝ - V.TRNKOVÁ - J.ADÁMEK

UNEXPECTED PROPERTIES OF LOCALLY PRESENTABLE CATEGORIES

ALGEBRA UNIVERSALIS 27 (1990), 153-170

[185]

A.SCHALK

DOMAINS ARISING AS ALGEBRAS FOR POWERSPACE CONSTRUCTIONS

JOURNAL OF PURE AND APPLIED ALGEBRA 89 (1993), 305-328

[186]

H.SCHUBERT

CATEGORIES

SPRINGER VERLAG (1972)

[187]

D.S.SCOTT

DOMAINS FOR DENOTATIONAL SEMANTICS

LECTURE NOTES IN COMPUTER SCIENCE 140 ,

SPRINGER VERLAG (1982), 577-613

[188]

A.SOLIAN - T.M.VISWANATHAN

PLURI-ADJOINTS AND PRESERVATION OF FINITE LIMITS 
JOURNAL OF PURE AND APPLIED ALGEBRA 65 (1990). 69-90

[189]

L.SOUSA

SOLID HULLS OF CONCRETE CATEGORIES

APPLIED CATEGORICAL STRUCTURES 3 (1995), 105-118

[190]

R.STREET

LIMITS INDEXED BY CATEGORY-VALUED 2-FUNCTORS

JOURNAL OF PURE AND APPLIED ALGEBRA 8 (1976), 149-181

[191]

R.STREET

FIBRATIONS IN BICATEGORIES

CAHIERS DE TOPOLOGIE ET GÉOMÉTRIE DIFFÉRENTIELLE

VOL.XXI (1980), 111-160

[192]

P.TAYLOR

QUANTITATIVE DOMAINS, GRUPOIDS AND LINEAR LOGIC

LECTURE NOTES IN COMPUTER SCIENCE 389,

SPRINGER VERLAG (1989), 155-181

[193]

P.TAYLOR

AN ALGEBRAIC APPROACH TO STABLE DOMAINS

JOURNAL OF PURE AND APPLIED ALGEBRA 64 (1990), 171-203

[194]

W.THOLEN

MACNEILLE COMPLETION OF CONCRETE CATEGORIES WITH LOCAL PROPERTIES

COMMENTARII MATHEMATICI UNIVERSITATIS SANCTI PAULI 28 (1979), 179202

[195]

W.THOLEN

PRO-CATEGORIES AND MULTIADJOINT FUNCTORS

CANADIAN JOURNAL OF MATHEMATICS VOL.XXXVI (1984). 144-155 [196]

W.THOLEN - A.TOZZI

COMPLETIONS OF CATEGORIES AND INITIAL COMPLETIONS

CAHIERS DE TOPOLOGIE ET GÉOMÉTRIE DIFFÉRENTIELLE 
CATÉGORIQUES

VOL.XXX (1989): 127-156

[197]

V.TRNKOVÁ

ALGEBRAIC THEORIES, CLONES AND THEIR SEGMENTS

APPLIED CATEGORICAL STRUCTURES 4 (1996): 241-249

[198]

F.ULMER

PROPERTIES OF DENSE AND RELATIVE ADJOINT FUNCTORS

JOURNAL OF ALGEBRA 8 (1968), 77-95

[199]

F.ULMER

REPRESENTABLE FUNCTORS WITH VALUES IN ARBITRARY CATEGORIES JOURNAL OF ALGEBRA 8 (1968), 96-129

[200]

F.ULMER

LOCALLY $\alpha$-PRESENTABLE AND LOCALLY $\alpha$-GENERATED

CATEGORIES

LECTURE NOTES IN MATHEMATICS 195, SPRINGER VERLAG (1971), 230-247

[201]

F.ULMER

ON THE EXISTENCE AND EXACTNESS OF THE ASSOCIATED

SHEAF FUNCTOR

JOURNAL OF PURE AND APPLIED ALGEBRA 3 (1973), 295-306

[202]

F.ULMER

ON CATEGORIES OF COCONTINUOUS FUNCTORS. APLICATIONS

CAHIERS DE TOPOLOGIE ET GÉOMÉTRIE DIFFÉRENTIELLE

VOL.XVI (1975), 325-330

[203]

M.P.VINCENT

CONSTRUCTION EXPLICITE D`UNE COMPLÉTION INDUCTIVE LIBRE

POUR UNE CATÉGORIE QUELCONQUE

COMPTES RENDUS DE L'ACADÉMIE DES SCIENCES (PARIS)

T.265, SERIE A (1967), 816-819

[204]

E.M.VITALE 
ON THE CHARACTERIZATION OF MONADIC CATEGORIES OVER SET CAHIERS DE TOPOLOGIE ET GÉOMÉTRIE DIFFÉRENTIELLE CATÉGORIQUES VOL.XXXV (1994), 351-358 [205]

E.M.VITALE LOCALIZATIONS OF ALGEBRAIC CATEGORIES JOURNAL OF PURE AND APPLIED ALGEBRA 108 (1996); 315-320 [206]

H.VOLGER

PRESERVATION THEOREMS FOR LIMITS OF STRUCTURES

AND GLOBAL SECTIONS OF SHEAVES OF STRUCTURES

MATHEMATISCHE ZEITSCHRIFT 166 (1979), 27-54

[207]

H.WEBERPALS

ÜBER EINEN SATZ VON GABRIEL ZUR

CHARAKTERISIERUNG REGULÄRER COLIMITES

MATHEMATISCHE ZEITSCHRIFT 161 (1978), 47-67

[208]

H.WEBERPALS

VERTAUSCHBARKEIT VON LIMITES UND COLIMITES

MANUSCRIPTA MATHEMATICA 49 (1984), 9-26

[209]

H.WEBERPALS

VOLLSTÄNDIGKEITSEIGENSCHAFTEN FREIER COLIMES-KOMPLETTIERUNGEN

ARCHIV DER MATHEMATIK 47 (1986), 151-158

[210]

C.WELLS - M.BARR

THE FORMAL DESCRIPTION OF DATA TYPES USING SKETCHES

LECTURE NOTES IN COMPUTER SCIENCE 298,

SPRINGER VERLAG (1988), 490-527

[211]

C.WELLS

A GENERALIZATION OF THE CONCEPT OF SKETCH THEORETICAL COMPUTER SCIENCE 70 (1990), 159-178

[212]

M.B.WISCHNEWSKY

ON TOPOLOGICAL ALGEBRAS RELATIVE TO 
FULL AND FAITHFUL DENSE FUNCTORS

LECTURE NOTES IN MATHEMATICS 540, SPRINGER VERLAG (1976), 688-698 